Schlumberger Soundings at the Amargosa Desert

Research Site, Nevada

by

Robert J. Bisdorf

Open-file Report 02-140

2002

This report has nor been reviewed for conformity with U.S. Geological Survey editorial standards. Any use of trade names is for descriptive purposes only and does not constitute endorsement by the USGS.

*U.S. Geological Survey, MS 964, Box 25046, Denver, CO 80225 


\title{
Schlumberger Soundings at the Amargosa Desert Research Site, Nevada
}

by

\author{
Robert J. Bisdorf
}

In 1999 the U.S. Geological Survey made 38 direct current (dc) electrical soundings at the Amargosa Desert Research Site (ADRS) near Beatty, Nevada (fig. 1.) using the Schlumberger array. An additional 16 Schlumberger soundings were made in 2000. The soundings were made to determine the subsurface resistivity distribution, and the location of faults. The purpose of this report is to present the data, its automatic interpretation, cross sections and resistivity depth maps.

Figure 2 is a map of the sounding locations. The soundings are represented by circles. The 1999 data are numbered 1 through 38 and the 2000 data are numbered 101 through 116 . Table 1 gives the sounding number, $x$ coordinate, and $y$ coordinate of the soundings in UTM zone 11 NAD27.

The data were interpreted using an automatic computerized interpretation program (Zohdy and Bisdorf, 1989) written for IBM PC's and compatible computers. The soundings are designated ADRS 1 through ADRS 116. For each sounding curve, the data in the appendix includes:

1) A sounding title designated by the name of the survey area followed by the sounding number. Several sounding titles have a suffix that indicates what special treatment the sounding may have undergone. The suffix "S" denotes that the field data were smoothed before the inversion was performed, a suffix " $F$ " indicates that the last layer resistivity was fixed at some value, and a suffix " $X$ " indicates that the sounding's S-line (Zohdy and others, 1974 p., 36) was manually extended. Any or all of the suffixes may be attached to the sounding number. In general, only the interpreted sounding titles will have suffixes.

2) A tabulation of the $A B / 2$ electrode spacings (in meters and feet) and corresponding apparent resistivities (in ohm-meters).

3) A log-log plot of the field data points. Each set of data points that were made with the same potential electrode spacing $(\mathrm{MN})$ is connected with a solid line. Measurements were made at MN/2 spacings of $0.6,2,20,60$, and $200 \mathrm{ft}$. as appropriate.

4) A tabulation of the automatically interpreted layering, with depths in meters and feet and the corresponding resistivities in ohm-meters.

5) A log-log plot of the results of the automatic interpretation program. The circles represent the shifted-digitized field data, the continuous curve represents the sounding curve calculated from the interpreted layering, and the step-function curve represents the interpreted layering.

\section{CROSS SECTIONS OF INTERPRETED RESISTIVITY}

Resistivity cross sections are generated from individual sounding interpretations. Each sounding interpretation is sampled in a manner to approximate a continuous vertical distribution of resistivity with depth (Bisdorf, 1982). These vertical distributions are then horizontally interpolated to create a grid. Colors are assigned based on the interpolated resistivity values 
and the desired contour levels. Triangles on the upper surface of the cross section designate the sounding locations. Topographic information, input as sounding elevations, is represented by connecting the surface location of the soundings by straight lines.

Figure 3 shows a cross section of interpreted resistivity (see fig. 2 for location). In the upper $30 \mathrm{~m}$. there is a horizontal layer of high resistivity $(45-200 \mathrm{ohm}-\mathrm{m})$. Between soundings 107 and 103 a fault is inferred. The black line indicates an approximate position for the inferred fault. The high resistivity zone at a depth of about $190 \mathrm{~m}$. under soundings 116 and 107 deepens to about $320 \mathrm{~m}$. under sounding 103, and the overlying low resistivity zone (<45 ohm$\mathrm{m}$ ) doubles in thickness on the down thrown side of the inferred fault.

\section{RESISTIVITY MAPS AT SELECTED DEPTHS}

Maps of interpreted resistivity at a particular elevation are generated by sampling the sounding interpretations at depths determined by the difference between the surface elevation at that sounding and the desired elevation. The resultant sampled interpreted resistivities and the corresponding location values are gridded using a minimum-curvature algorithm (Webring, 1981). To prevent possible interpolated resistivities of less than zero, the logarithm of the resistivities is used for gridding. Colors are assigned based on the grid value and the desired logarithmically spaced contour levels. Since these maps are raster (pixel) based, a bicubic interpolation program is used to increase the size of the resultant image. An interpolating program is used to resample the grid, as opposed to simply gridding the data at the desired final interval, because the minimum-curvature algorithm generates undesirable results if the data are over sampled. The Kolor-map and section program (Zohdy, 1993) uses similar procedures and provides a discussion of the nuances of resistivity map generation.

Figure 4 shows a map of interpreted resistivity at a depth of $97 \mathrm{~m}$. The major features on the map include:

a) Low resistivity( $<15 \mathrm{ohm}-\mathrm{m})$ zone just to the southeast of the disposal site. Possibly indicative of increased clay content or increased water content.

b) High resistivity zones on the eastern side of the map possibly indicating the presence of Quartzite.

c) Linear resistivity contours separating the zones of high resistivity from the zones of lower resistivity. These linear trends may indicate the presence of faults.

Ninety seven $\mathrm{m}$. was chosen because deeper maps would only have about $1 / 2$ of the sounding locations of this map. This is because in the initial electrical resistivity survey the soundings were only expanded to an $A B / 2$ of 800 to $1000 \mathrm{ft}$.

\section{Summary}

The interpretation of dc resistivity soundings were used to create an interpreted resistivity cross section and a map of interpreted resistivity at a depth of $97 \mathrm{~m}$. Faults are inferred from the cross section and the depth map. Because of the limited number of soundings, the location and geometry of the faults are not well defined. 


\section{REFERENCES}

Bisdorf, R.J., 1982, Schlumberger sounding investigations in the Date Creek Basin, Arizona: U.S. Geological Survey Open-File Report 82-953, 55 p.

Lucius, J. E., and Bisdorf, R. J., 1995, Results of geophysical investigations near the Norman, Oklahoma, municipal landfill, 1995: U.S. Geological Survey Open-File report 95-825, $125 \mathrm{p}$.

Webring, Michael, 1981, MINC: A gridding program based on minimum curvature: U.S. Geological Survey Open-File Report 81-1224, 12 p.

Zohdy, A. A. R., 1993, Program Kolor-Map \& Section, Amiga Version: U.S. Geological Survey Open-File Report 93-585, 113 p.

Zohdy, A. A. R., and Bisdorf, R. J., 1989, Programs for the automatic processing and interpretation of Schlumberger sounding curves in QuickBASIC 4.0: U.S. Geological Survey Open-File Report, 89-137 A\&B, 64 p. + diskette.

Zohdy, A. A. R., Eaton, G.P., and Mabey, D. R., 1974, Application of surface geophysics to ground-water investigations: Techniques of water-resources investigations of the United States Geological Survey, Book 2, Chapter D1, 116 p. 


\begin{tabular}{|r|r|r|r|r|r|}
\hline $\begin{array}{c}\text { Sounding } \\
\text { Number }\end{array}$ & $\begin{array}{c}\mathrm{X} \\
\text { coordinate } \\
(\mathrm{km})\end{array}$ & $\begin{array}{c}\mathrm{Y} \\
\text { coordinate } \\
(\mathrm{km})\end{array}$ & $\begin{array}{c}\text { Sounding } \\
\text { Number }\end{array}$ & $\begin{array}{c}\mathrm{X} \\
\text { coordinate } \\
(\mathrm{km})\end{array}$ & $\begin{array}{c}\mathrm{Y} \\
\text { coordinate } \\
(\mathrm{km})\end{array}$ \\
\hline & & & & & \\
\hline 1 & 527.081 & 4068.750 & 28 & 527.516 & 4068.740 \\
\hline 2 & 527.331 & 4068.450 & 29 & 527.321 & 4068.740 \\
\hline 3 & 528.755 & 4068.491 & 30 & 529.866 & 4070.887 \\
\hline 4 & 529.050 & 4068.495 & 31 & 528.111 & 4068.740 \\
\hline 5 & 527.831 & 4068.650 & 32 & 528.416 & 4068.729 \\
\hline 6 & 527.031 & 4068.650 & 33 & 528.031 & 4068.450 \\
\hline 7 & 527.231 & 4068.650 & 34 & 528.031 & 4068.230 \\
\hline 8 & 527.431 & 4068.650 & 35 & 528.031 & 4067.987 \\
\hline 9 & 527.631 & 4068.650 & 36 & 527.731 & 4068.350 \\
\hline 10 & 527.831 & 4068.450 & 37 & 527.731 & 4068.106 \\
\hline 11 & 527.631 & 4068.450 & 38 & 528.231 & 4068.230 \\
\hline 12 & 527.431 & 4068.450 & 101 & 526.499 & 4068.853 \\
\hline 13 & 527.231 & 4068.450 & 102 & 527.219 & 4069.154 \\
\hline 14 & 527.031 & 4068.450 & 103 & 528.050 & 4069.162 \\
\hline 15 & 526.831 & 4068.450 & 104 & 527.676 & 4069.165 \\
\hline 16 & 528.031 & 4068.650 & 105 & 528.050 & 4068.908 \\
\hline 17 & 529.120 & 4068.595 & 106 & 528.235 & 4068.922 \\
\hline 18 & 528.168 & 4068.650 & 107 & 528.520 & 4069.180 \\
\hline 19 & 527.306 & 4068.700 & 108 & 529.293 & 4070.102 \\
\hline 20 & 527.931 & 4068.550 & 109 & 528.755 & 4069.464 \\
\hline 21 & 527.731 & 4068.550 & 110 & 526.850 & 4069.115 \\
\hline 22 & 527.531 & 4068.550 & 111 & 527.219 & 4068.967 \\
\hline 23 & 528.131 & 4068.550 & 112 & 529.660 & 4068.760 \\
\hline 24 & 528.331 & 4068.550 & 113 & 526.840 & 4068.330 \\
\hline 25 & 528.531 & 4068.550 & 114 & 527.649 & 4069.521 \\
\hline 26 & 527.911 & 4068.740 & 115 & 528.000 & 4069.690 \\
\hline 27 & 527.711 & 4068.740 & 116 & 529.143 & 4069.201 \\
\hline & & & & & \\
\hline & & & & \\
\hline & & & & & \\
\hline & & & & & \\
\hline
\end{tabular}

Table 1. Sounding number and $\mathrm{x}-\mathrm{y}$ coordinates in $\mathrm{km}$ based on UTM zone 11, 1927 North American Datum. 


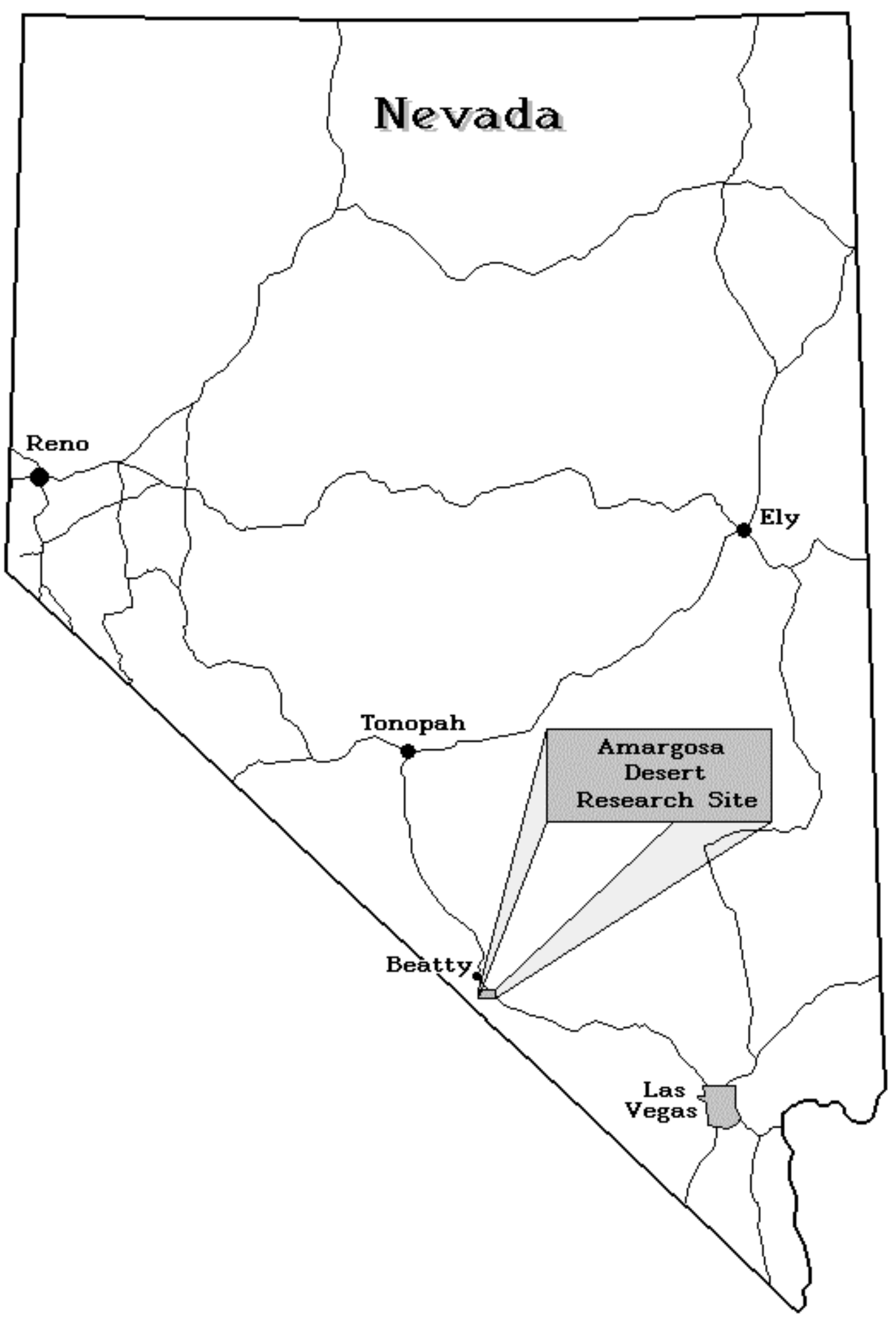

Figure 1. Location of the Amargosa Desert Research Site. 


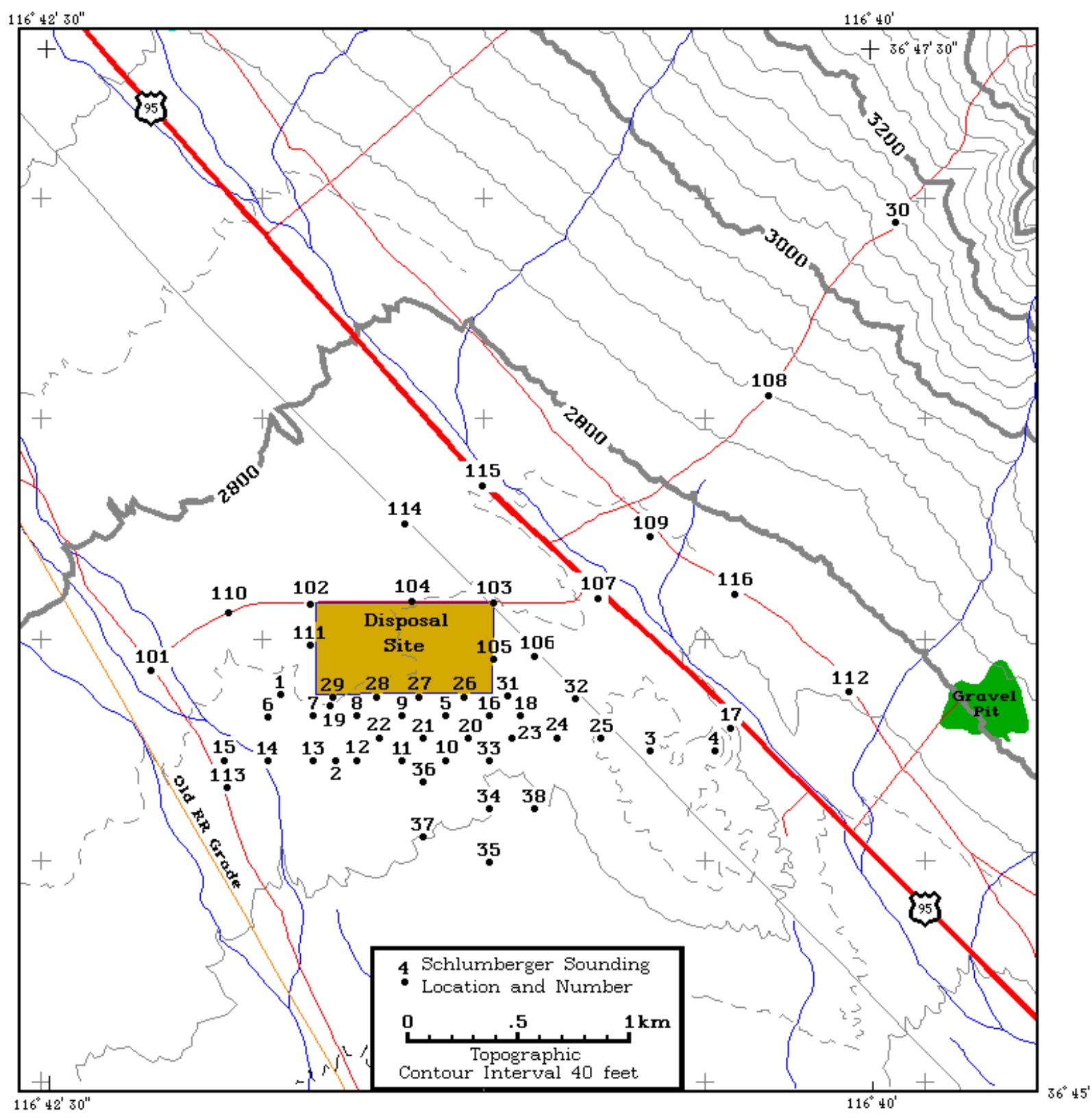

Figure 2. Map showing the number and location of the Schlumberger soundings. 


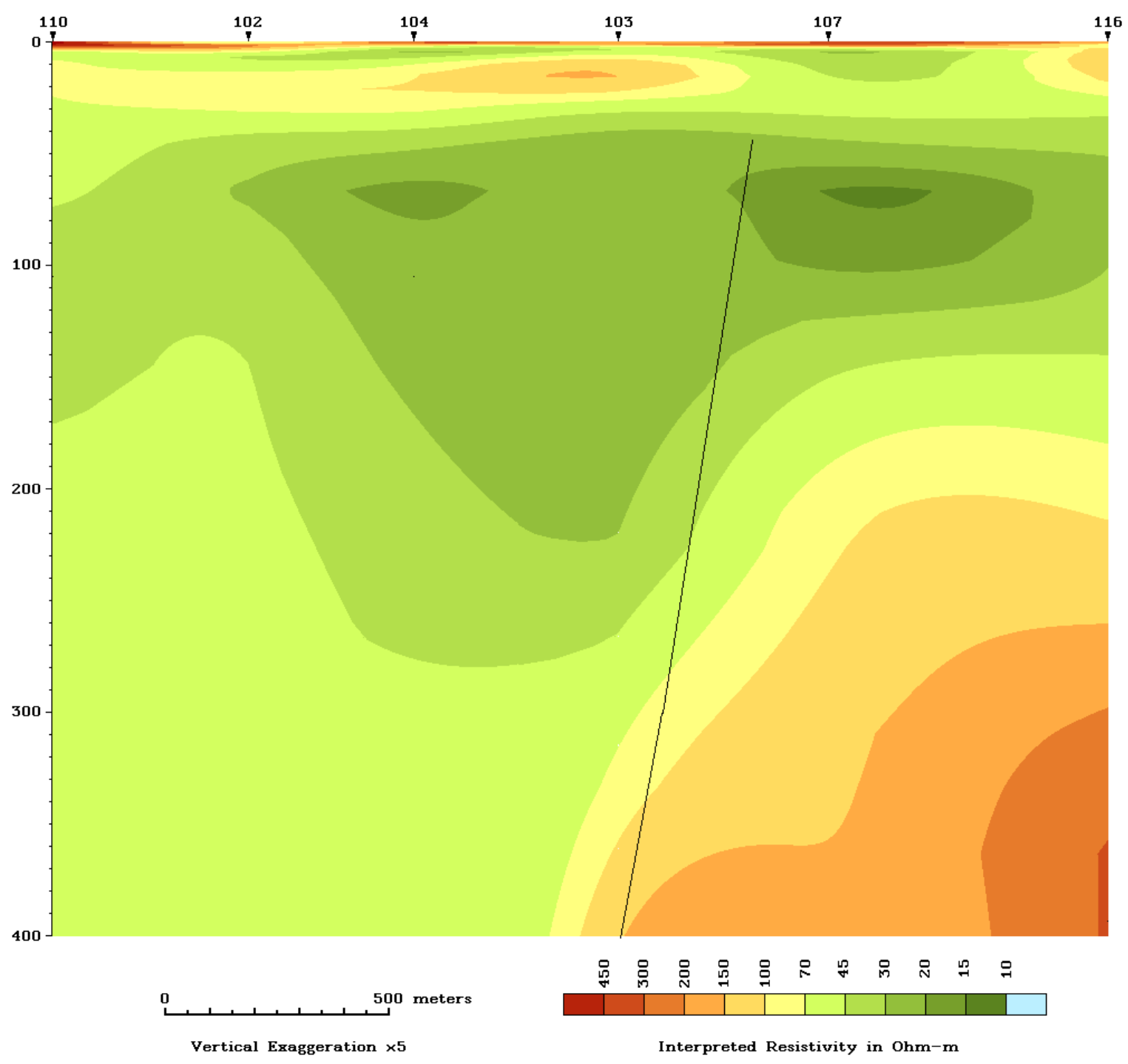

Figure 3. Cross section of interpreted resistivity. Triangles represent Schlumberger sounding locations. Colors represent interpreted resistivity values. 


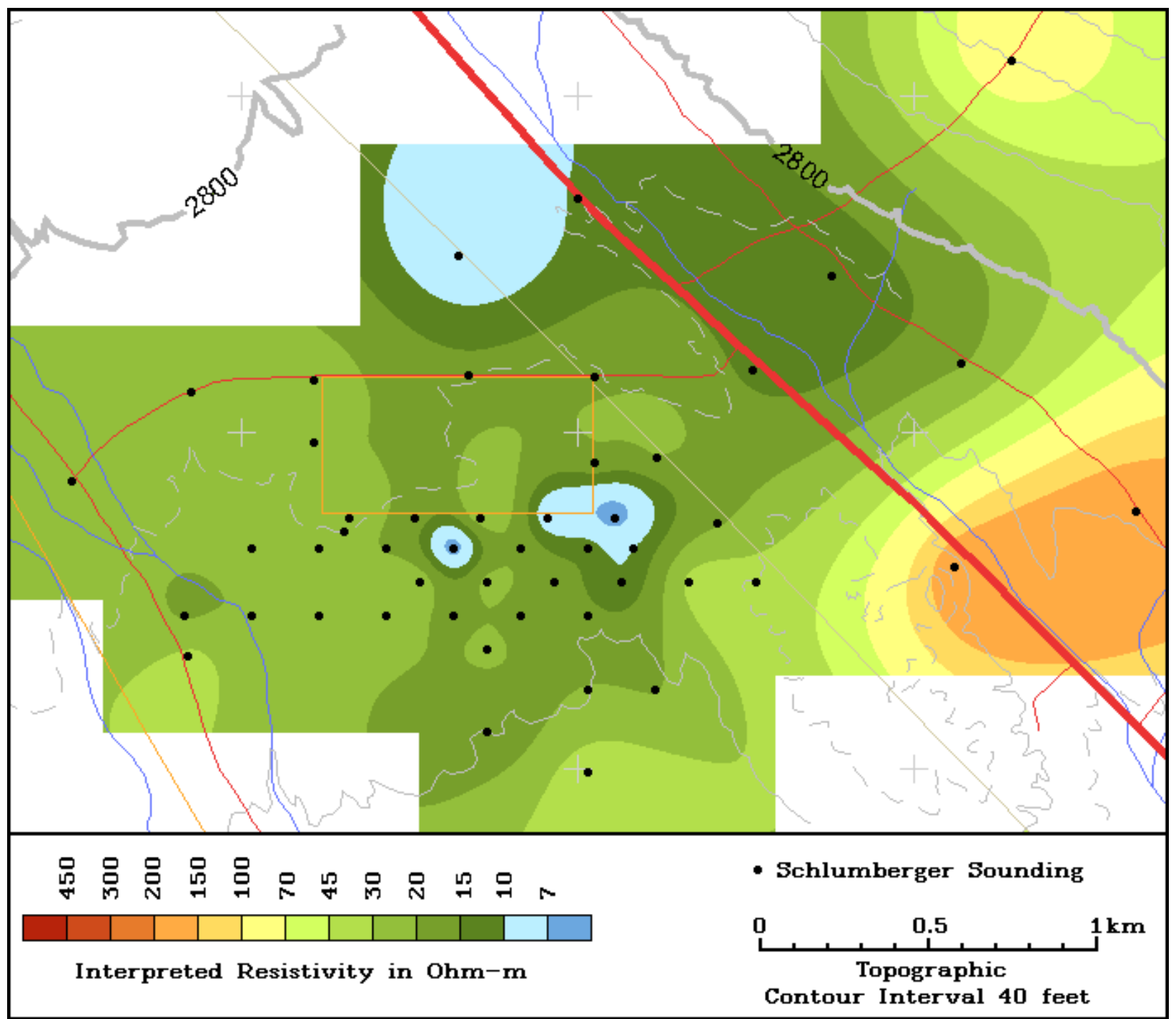

Figure 4. Map showing contours of interpreted resistivity at a depth of $97 \mathrm{~m}$ overlying topographic contours. 
APPENDIX 


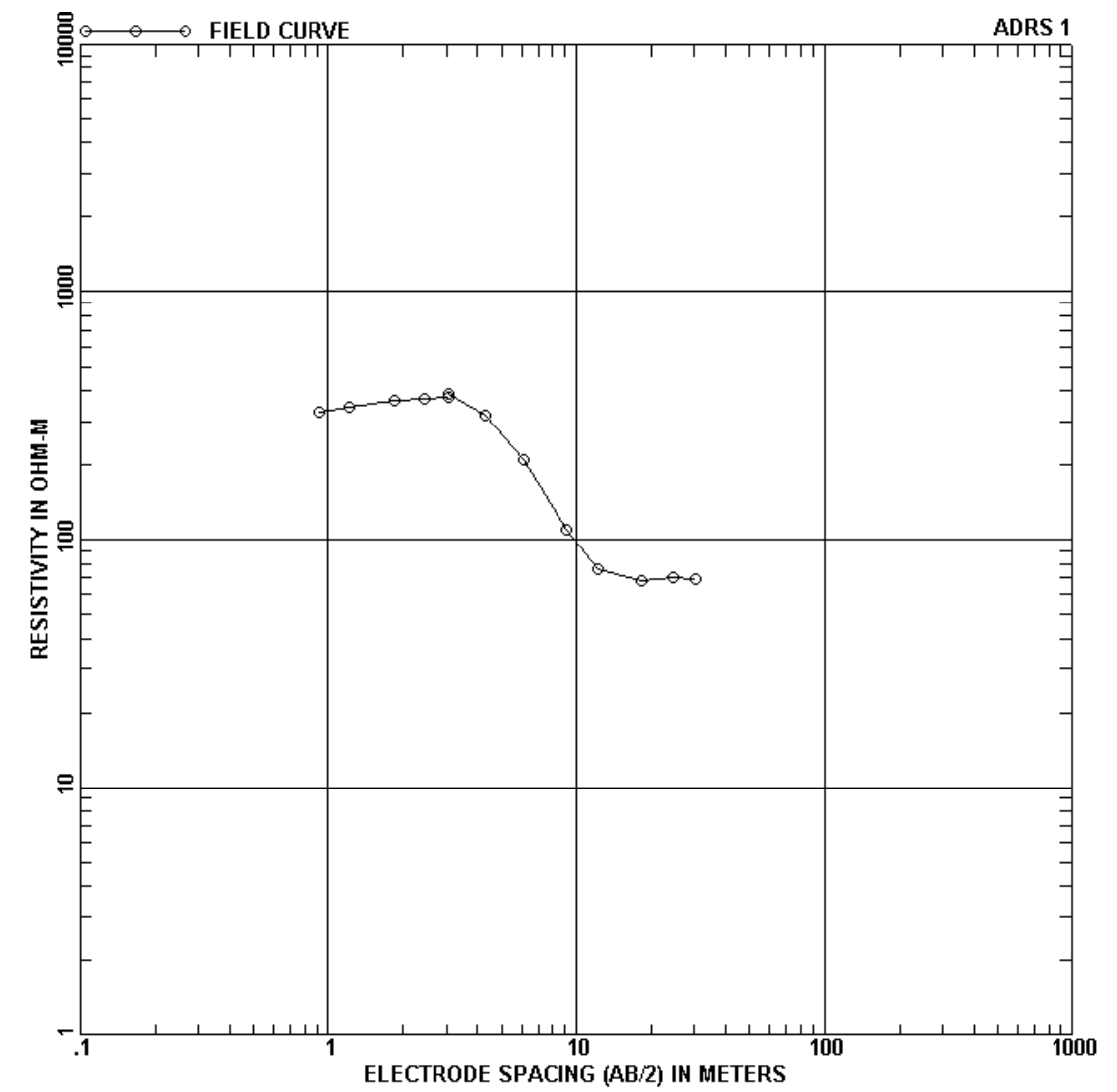

$A B / 2$, m ( ft ) App. Res. $A B / 2$, m ( ft ) App. Res.

\begin{tabular}{|c|c|c|c|c|c|}
\hline 0.91 & $3.00)$ & 329.00 & 4.27 & $14.00)$ & 317.00 \\
\hline 1.22 & $4.00)$ & 344.00 & 6.10 & $20.00)$ & 208.00 \\
\hline 1.83 & $6.00)$ & 367.00 & 9.14 & $30.00)$ & 110.00 \\
\hline 2.44 & $8.00)$ & 371.00 & 12.19 & $40.00)$ & 75.90 \\
\hline 3.05 & $10.00)$ & 375.00 & 18.29 & $60.00)$ & 68.40 \\
\hline 3.05 & $10.00)$ & 389.00 & 24.38 & $80.00)$ & 70.70 \\
\hline & & & 30.48 & $100.00)$ & 69.10 \\
\hline
\end{tabular}

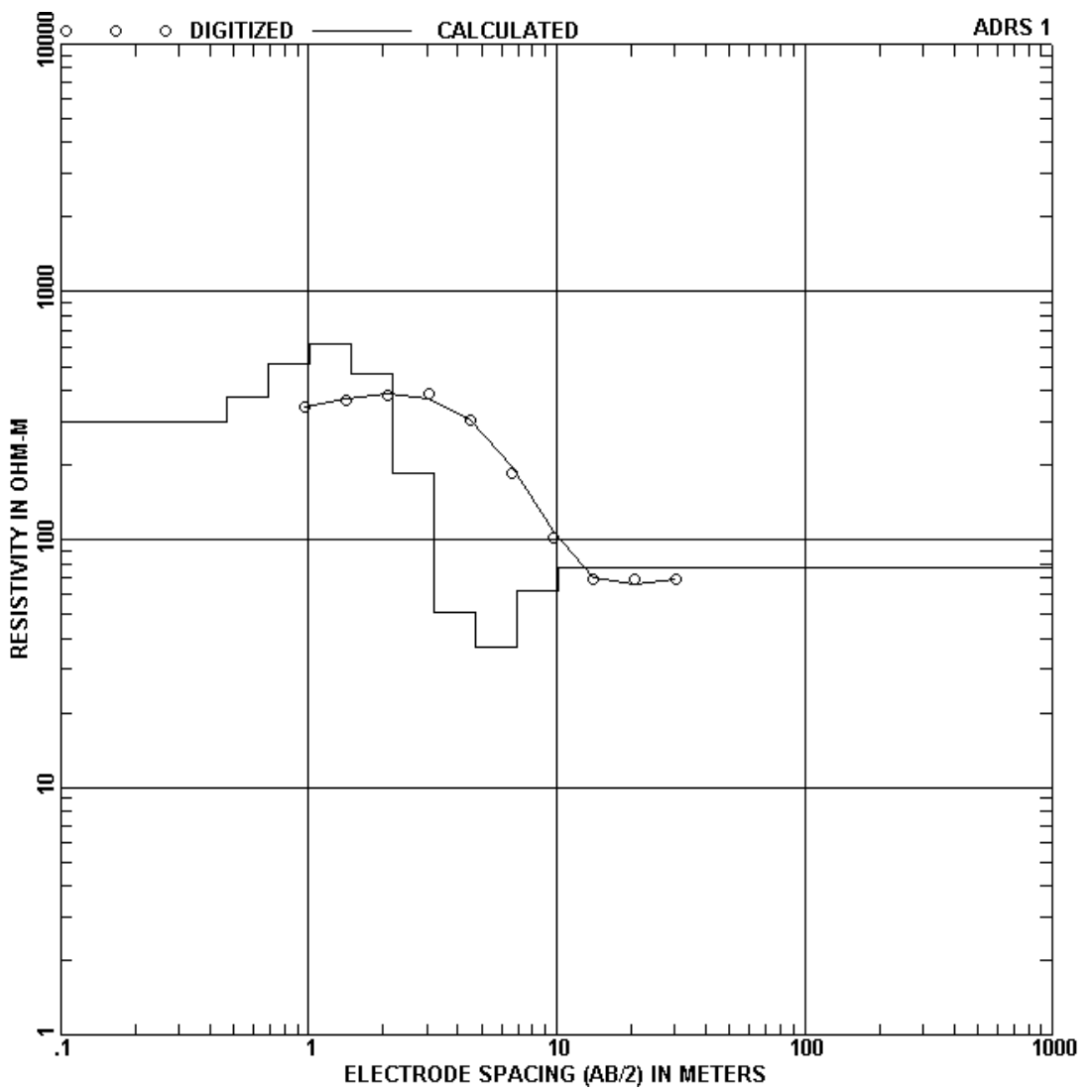

DEPTH, $m$ ( ft ) RESIS. DEPTH, m ( ft ) RESIS.

$\begin{array}{rrrrrr}0.47 & (1.54) & 300.55 & 3.19( & 10.47) & 184.39 \\ 0.69( & 2.26) & 373.28 & 4.68( & 15.37) & 50.72 \\ 1.01( & 3.31) & 514.87 & 6.88( & 22.56) & 36.88 \\ 1.48( & 4.86) & 617.03 & 10.09(13.11) & 61.97 \\ 2.17( & 7.13) & 467.72 & 99999.00 & (99999.00) & 77.56\end{array}$




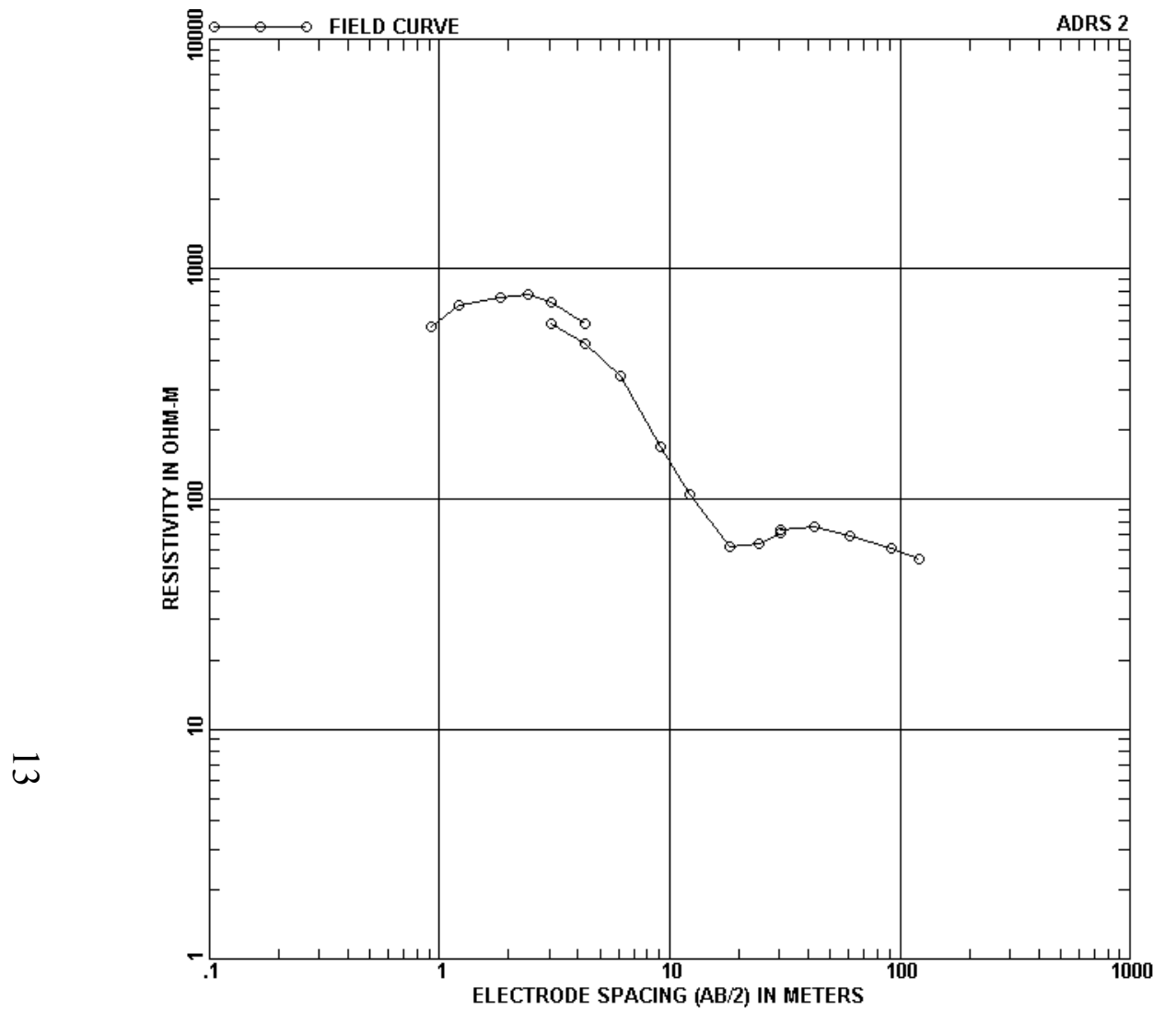

$A B / 2, m(f t)$ App. Res. $A B / 2, m(f t)$ App. Res.

\begin{tabular}{|c|c|c|}
\hline 0.91 & $3.00)$ & 62.00 \\
\hline 1.22 & $4.00)$ & 691.00 \\
\hline 1.83 & $6.00)$ & 747.00 \\
\hline 2.44 & $8.00)$ & 770.00 \\
\hline 3.05 & $10.00)$ & 719.00 \\
\hline 4.27 & $14.00)$ & 583.00 \\
\hline 3.05 & $10.00)$ & 580.00 \\
\hline 4.27 & $14.00)$ & 476.00 \\
\hline 6.10 & $20.00)$ & 341.00 \\
\hline
\end{tabular}

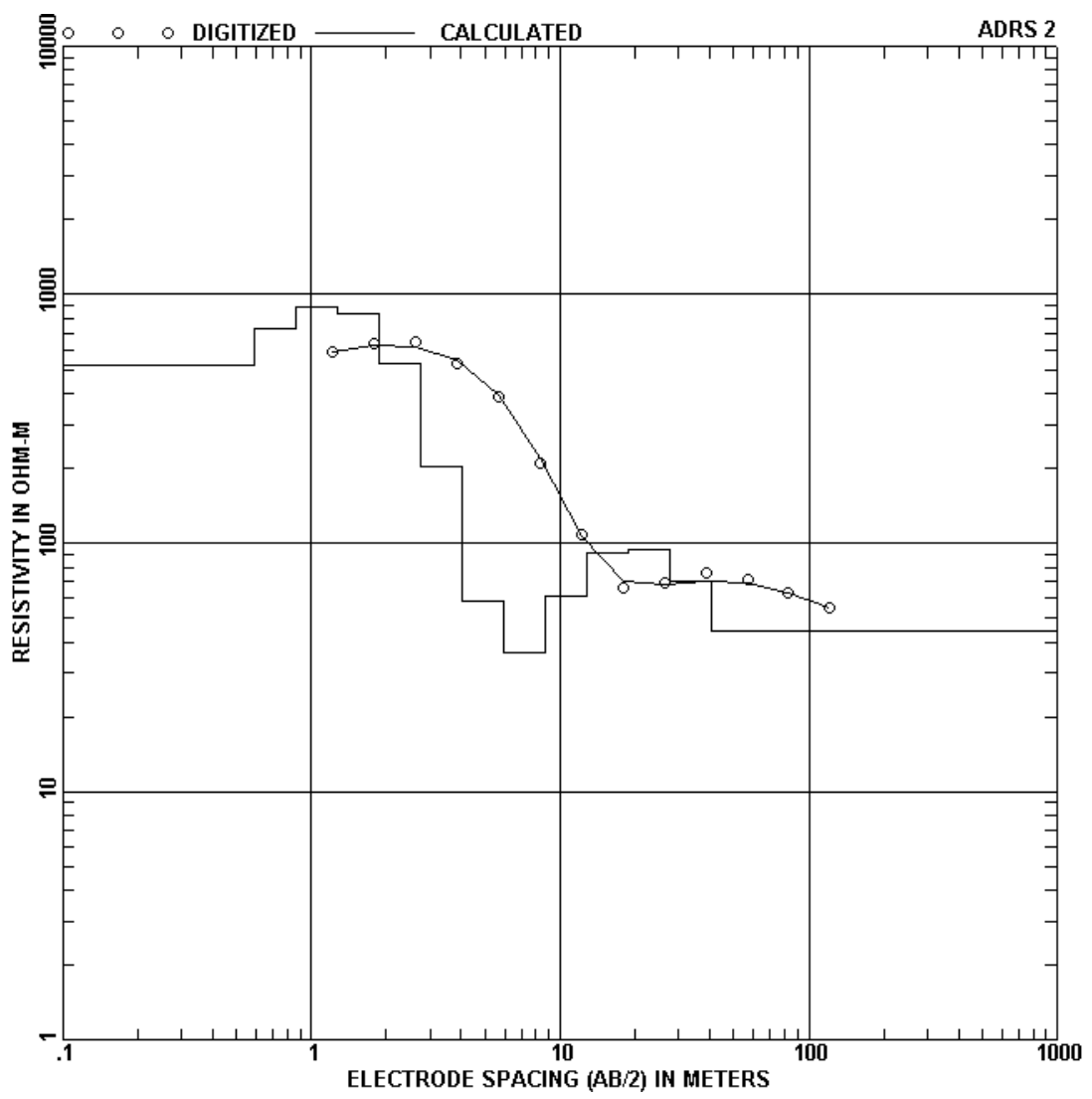

DEPTH, m ( ft ) RESIS. DEPTH, m ( ft ) RESIS.

\begin{tabular}{|c|c|c|c|c|c|}
\hline 0.59 & 1.94) & 517.15 & 5.93 & $19.44)$ & 58.43 \\
\hline 0.87 & $2.85)$ & 730.40 & 8.70 & $28.53)$ & 36.49 \\
\hline 1.28 & $4.19)$ & 896.30 & 12.77 & $41.88)$ & 60.98 \\
\hline 1.87 & $6.15)$ & 832.20 & 18.74 & $61.47)$ & 91.39 \\
\hline 2.75 & $9.02)$ & 524.13 & 27.50 & $90.23)$ & 93.76 \\
\hline \multirow[t]{2}{*}{4.04} & $13.24)$ & 203.44 & 40.37 & $132.44)$ & 69.75 \\
\hline & & & 99999.00 & $(99999.00)$ & 44.25 \\
\hline
\end{tabular}



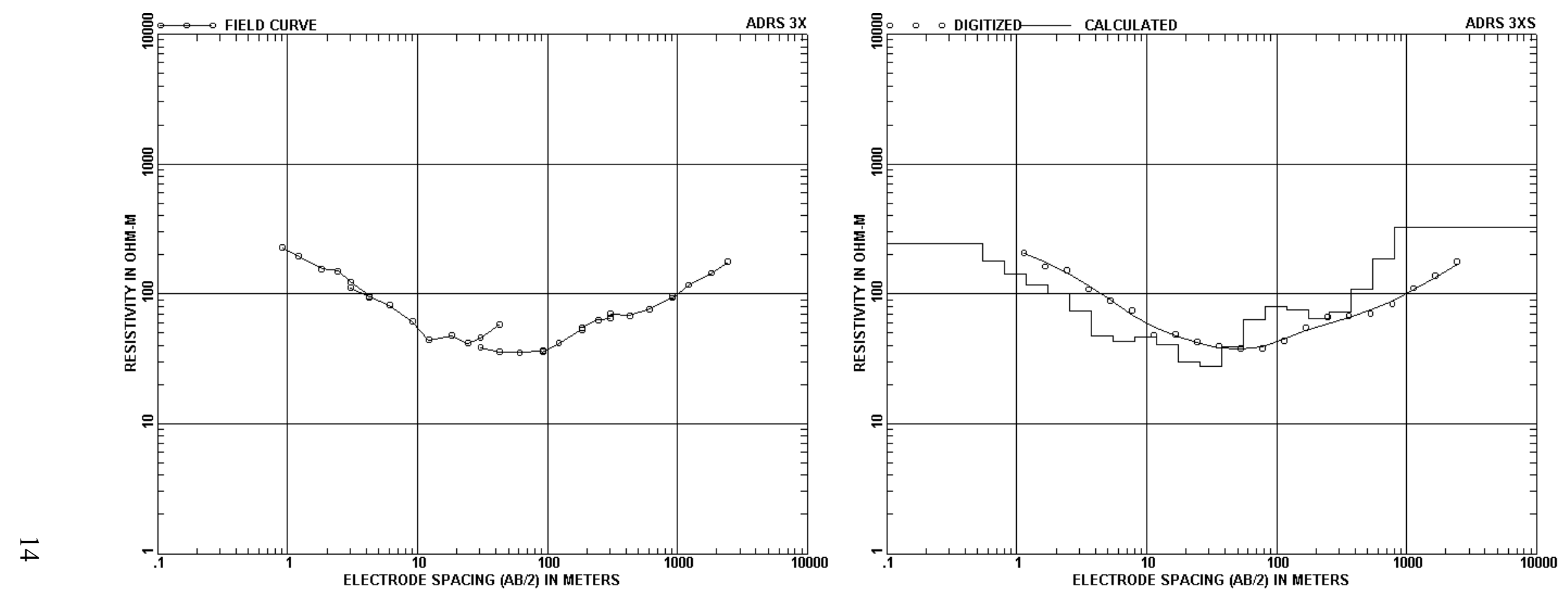

$A B / 2, m$ ( ft ) App. Res. $A B / 2$, m ( ft ) App. Res.

DEPTH, $m$ ( ft ) RESIS. DEPTH, m ( ft ) RESIS.

\begin{tabular}{|c|c|c|c|c|c|}
\hline 0.91 & $3.00)$ & 227.00 & 42.67 & $(140.00)$ & 35.70 \\
\hline 1.22 & $4.00)$ & 195.00 & 60.96 & $200.00)$ & 35.30 \\
\hline 1.83 & $6.00)$ & 156.00 & 91.44 & $300.00)$ & 36.50 \\
\hline 2.44 & $8.00)$ & 150.00 & 91.44 & $300.00)$ & 35.60 \\
\hline 3.05 & $10.00)$ & 123.00 & 121.92 & $(400.00)$ & 41.60 \\
\hline 4.27 & $14.00)$ & 96.00 & 182.88 & $600.00)$ & 52.70 \\
\hline 3.05 & $10.00)$ & 111.00 & 182.88 & $600.00)$ & 55.00 \\
\hline 4.27 & $14.00)$ & 94.00 & 243.84 & $800.00)$ & 63.00 \\
\hline 6.10 & $20.00)$ & 81.70 & 304.80 & $(1000.00)$ & 65.00 \\
\hline 9.14 & $30.00)$ & 61.40 & 304.80 & $(1000.00)$ & 70.00 \\
\hline 12.19 & $40.00)$ & 44.20 & 426.72 & $(1400.00)$ & 68.00 \\
\hline 18.29 & $60.00)$ & 47.80 & 609.60 & $(2000.00)$ & 76.00 \\
\hline 24.38 & $80.00)$ & 41.60 & 914.40 & $(3000.00)$ & 95.00 \\
\hline 30.48 & $100.00)$ & 46.00 & 914.40 & $(3000.00)$ & 93.00 \\
\hline 42.67 & $140.00)$ & 58.40 & 1219.20 & $(4000.00)$ & 117.00 \\
\hline \multirow[t]{2}{*}{30.48} & $100.00)$ & 38.50 & 1828.80 & $(6000.00)$ & 145.00 \\
\hline & & & 2438.40 & $8000.00)$ & 177.00 \\
\hline
\end{tabular}

\begin{tabular}{|c|c|}
\hline 0.55 & $1.80)$ \\
\hline 0.81 & $(2.65)$ \\
\hline 1.19 & $3.89)$ \\
\hline 1.74 & $5.71)$ \\
\hline 2.55 & $8.38)$ \\
\hline 3.75 & $12.29)$ \\
\hline 5.50 & $18.05)$ \\
\hline 8.07 & $26.49)$ \\
\hline 11.85 & $38.88)$ \\
\hline 17.39 & $57.07)$ \\
\hline
\end{tabular}

245.39

179.54

142.80

116.74

101.03

74.23

47.50

43.35

46.72

40.64

$25.53(83.76)$

$37.47(122.95)$

$55.01(180.47)$

80.74 ( 264.89)

118.51 ( 388.80$)$

173.94 ( 570.68$)$

374.75 ( 1229.49$)$

550.06 (1804.65)

807.37 ( 2648.86)

99999.00 (99999.00)

29.85

27.50

39.27

63.25

80.41

75.13

64.74

71.71

71.71
108.44

108.44

186.24 


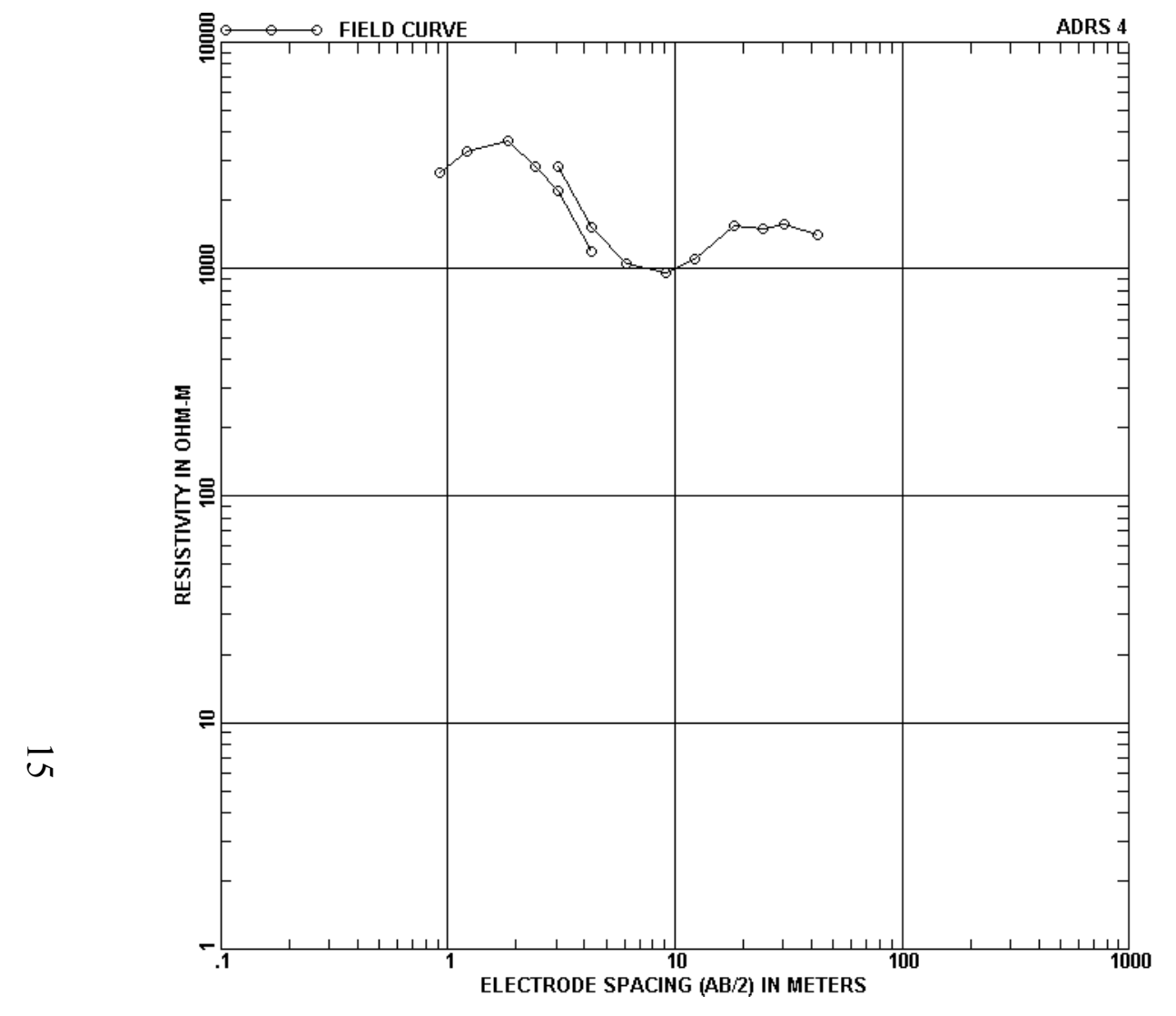

$A B / 2$, $m$ ( ft ) App. Res. $A B / 2$, $m$ ( ft ) App. Res.

\begin{tabular}{|c|c|c|c|c|c|}
\hline 0.91 & $3.00)$ & 2666.00 & 4.27 & $14.00)$ & 1527.00 \\
\hline 1.22 & $4.00)$ & 3289.00 & 6.10 & $20.00)$ & 1060.00 \\
\hline 1.83 & $6.00)$ & 3689.00 & 9.14 & $30.00)$ & 969.00 \\
\hline 2.44 & $8.00)$ & 2814.00 & 12.19 & $40.00)$ & 1104.00 \\
\hline 3.05 & $10.00)$ & 2210.00 & 18.29 & $60.00)$ & 1539.00 \\
\hline 4.27 & $14.00)$ & 1189.00 & 24.38 & $80.00)$ & 1492.00 \\
\hline 3.05 & $10.00)$ & 2833.00 & $\begin{array}{l}30.48 \\
42.67\end{array}$ & $\begin{array}{l}100.00) \\
140.00)\end{array}$ & $\begin{array}{l}1586.00 \\
1416.00\end{array}$ \\
\hline
\end{tabular}

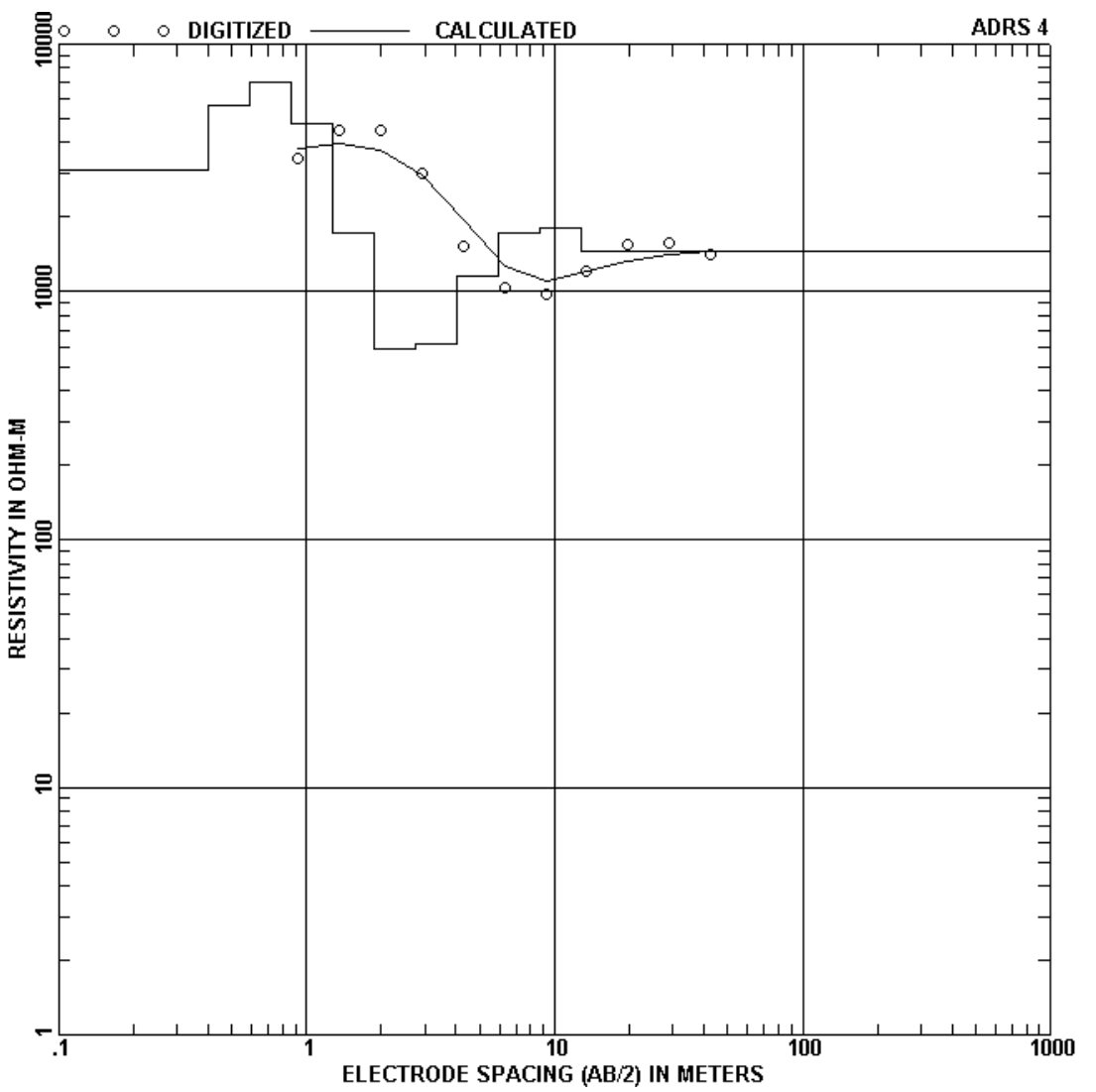

DEPTH, $m$ ( ft ) RESIS. DEPTH, m ( ft ) RESIS.

\begin{tabular}{|c|c|c|c|c|c|}
\hline 0.401 & 1. 32) & 3076.99 & 2.74 & $8.99)$ & 591.05 \\
\hline 0.591 & $1.94)$ & 5680.30 & 4.02 & 13.19) & 617.39 \\
\hline $0.87 \quad$ & $2.84)$ & 6983.81 & 5.90 & 19.36) & 1152.72 \\
\hline $1.27 \quad$ & 4.17) & 4788.29 & 8.66 & 28.42) & 1714.99 \\
\hline 1.87( & $6.12)$ & 1720.84 & $\begin{array}{r}12.72 \\
99999.00\end{array}$ & $\begin{array}{r}(41.72) \\
(99999.00)\end{array}$ & $\begin{array}{l}1797.17 \\
1452.69\end{array}$ \\
\hline
\end{tabular}




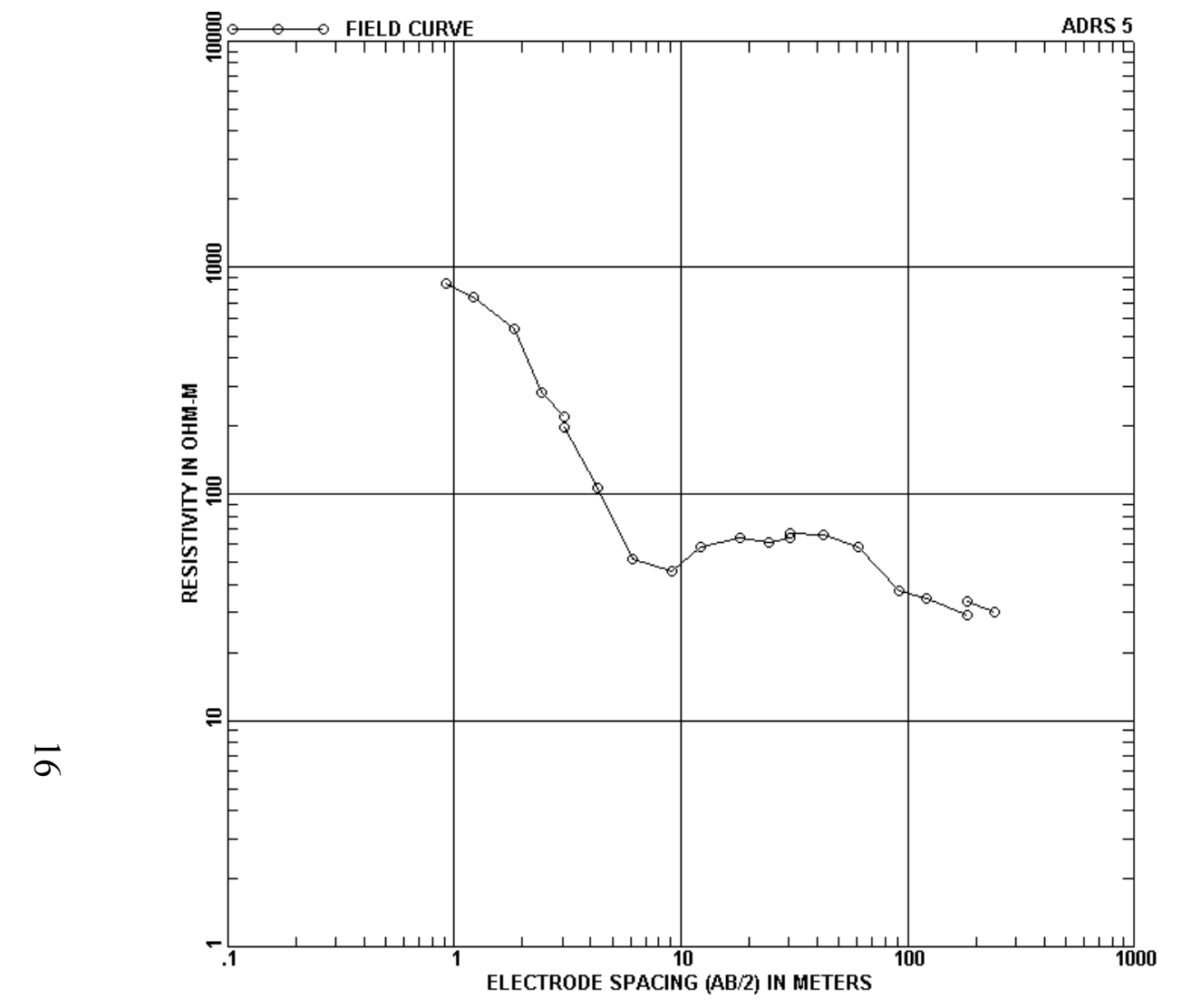

$A B / 2, m(f t)$ App. Res. $A B / 2, m$ ( ft ) App. Res.

\begin{tabular}{|c|c|c|}
\hline 0.91 & $3.00)$ & 855.00 \\
\hline 1.22 & $4.00)$ & 739.00 \\
\hline 1.83 & $6.00)$ & 534.00 \\
\hline 2.44 & $8.00)$ & 281.00 \\
\hline 3.05 & $10.00)$ & 218.00 \\
\hline 3.05 & $10.00)$ & 198.00 \\
\hline 4.27 & $14.00)$ & 107.00 \\
\hline 6.10 & $20.00)$ & 52.00 \\
\hline 9.14 & $30.00)$ & 45.60 \\
\hline 12.19 & $40.00)$ & 58.50 \\
\hline
\end{tabular}

\begin{tabular}{|c|c|c|}
\hline 18.29 & $60.00)$ & 63.70 \\
\hline 24.38 & $80.00)$ & 60.90 \\
\hline 30.48 & $100.00)$ & 63.80 \\
\hline 30.48 & $100.00)$ & 66.7 \\
\hline 42.67 & $140.00)$ & 65.80 \\
\hline 60.96 & $200.00)$ & 58.80 \\
\hline 91.44 & $300.00)$ & 37.50 \\
\hline 21.92 & $400.00)$ & 34.80 \\
\hline 28 & $600.00)$ & 29.00 \\
\hline .8 & $600.00)$ & 33.40 \\
\hline 13.8 & $800.00)$ & 29.9 \\
\hline
\end{tabular}

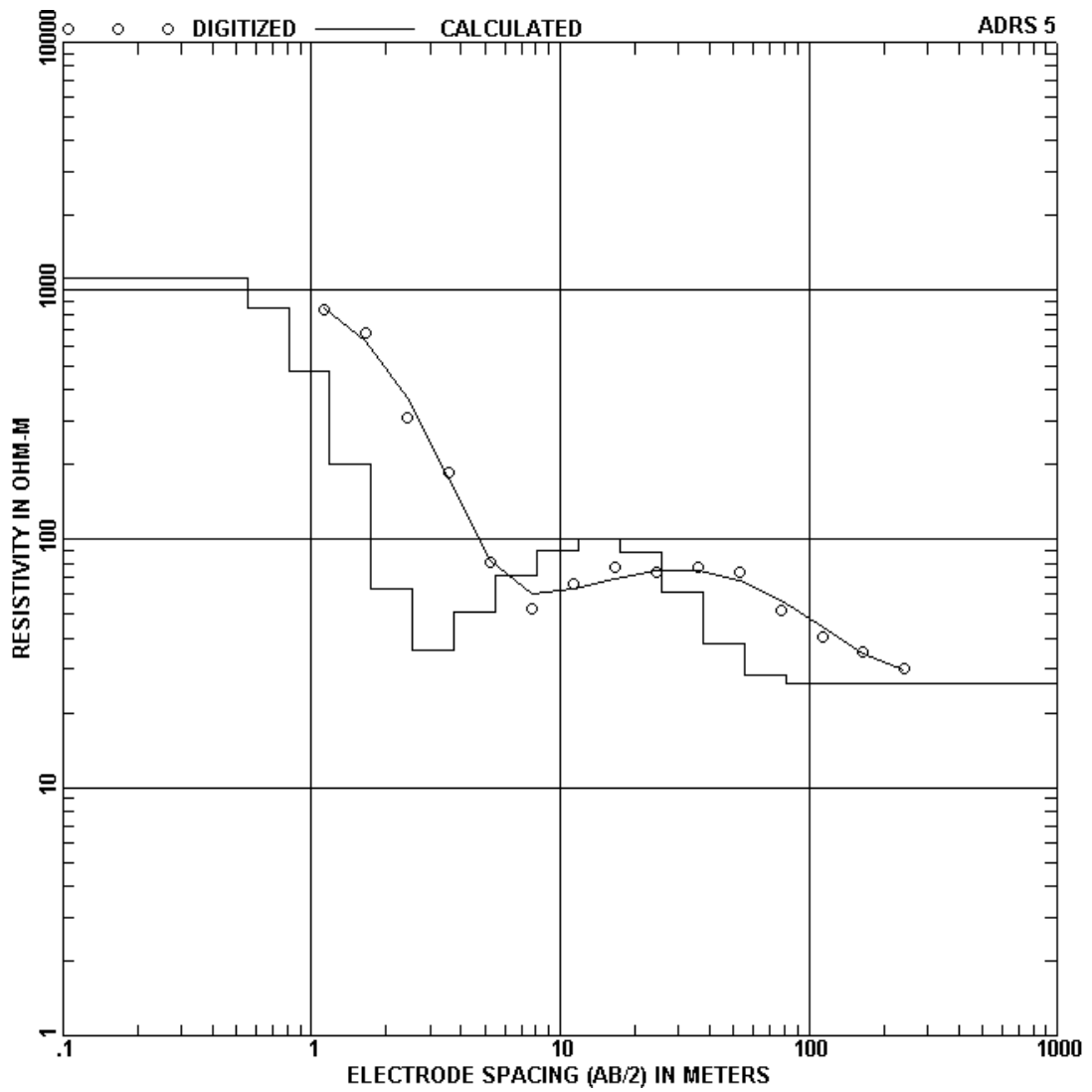

DEPTH, m ( ft ) RESIS. DEPTH, m ( ft ) RESIS.

$\begin{array}{rlr}0.55 & ( & 1.80) \\ 0.81 & ( & 2.65) \\ 1.19 & ( & 3.89) \\ 1.74 & ( & 5.71) \\ 2.55 & ( & 8.38) \\ 3.75 & ( & 12.29) \\ 5.50 & ( & 18.05)\end{array}$

845.50

471.77

201.04

63.05 35.46

50.50
71.77

89.96

99.83

88.93

60.82

37.92

28.37

26.08 


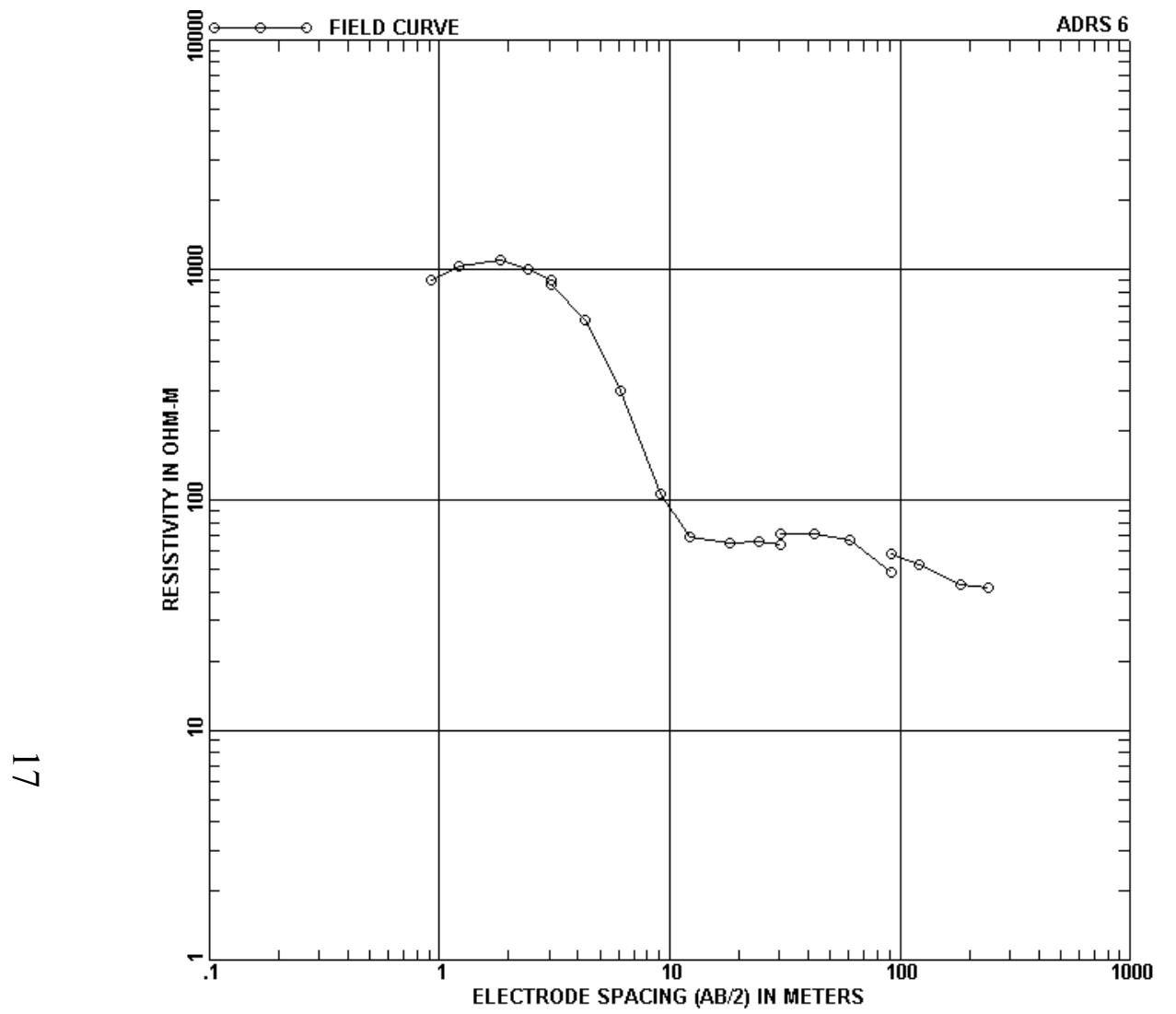

$\mathrm{AB} / 2$, $\mathrm{m}$ ( ft ) App. Res. $\mathrm{AB} / 2$, $\mathrm{m}$ ( ft ) App. Res.

\begin{tabular}{|c|c|c|}
\hline 0.91 & $3.00)$ & 907.00 \\
\hline 1.22 & $4.00)$ & 1045.00 \\
\hline 1.83 & $6.00)$ & 1102.00 \\
\hline 2.44 & $8.00)$ & 1014.00 \\
\hline 3.05 & $10.00)$ & 898.00 \\
\hline 3.05 & $10.00)$ & 58.00 \\
\hline 4.27 & $14.00)$ & 607.00 \\
\hline 6.10 & $20.00)$ & 297.00 \\
\hline 9.14 & $30.00)$ & 107.00 \\
\hline 12.19 & $40.00)$ & 69.30 \\
\hline
\end{tabular}

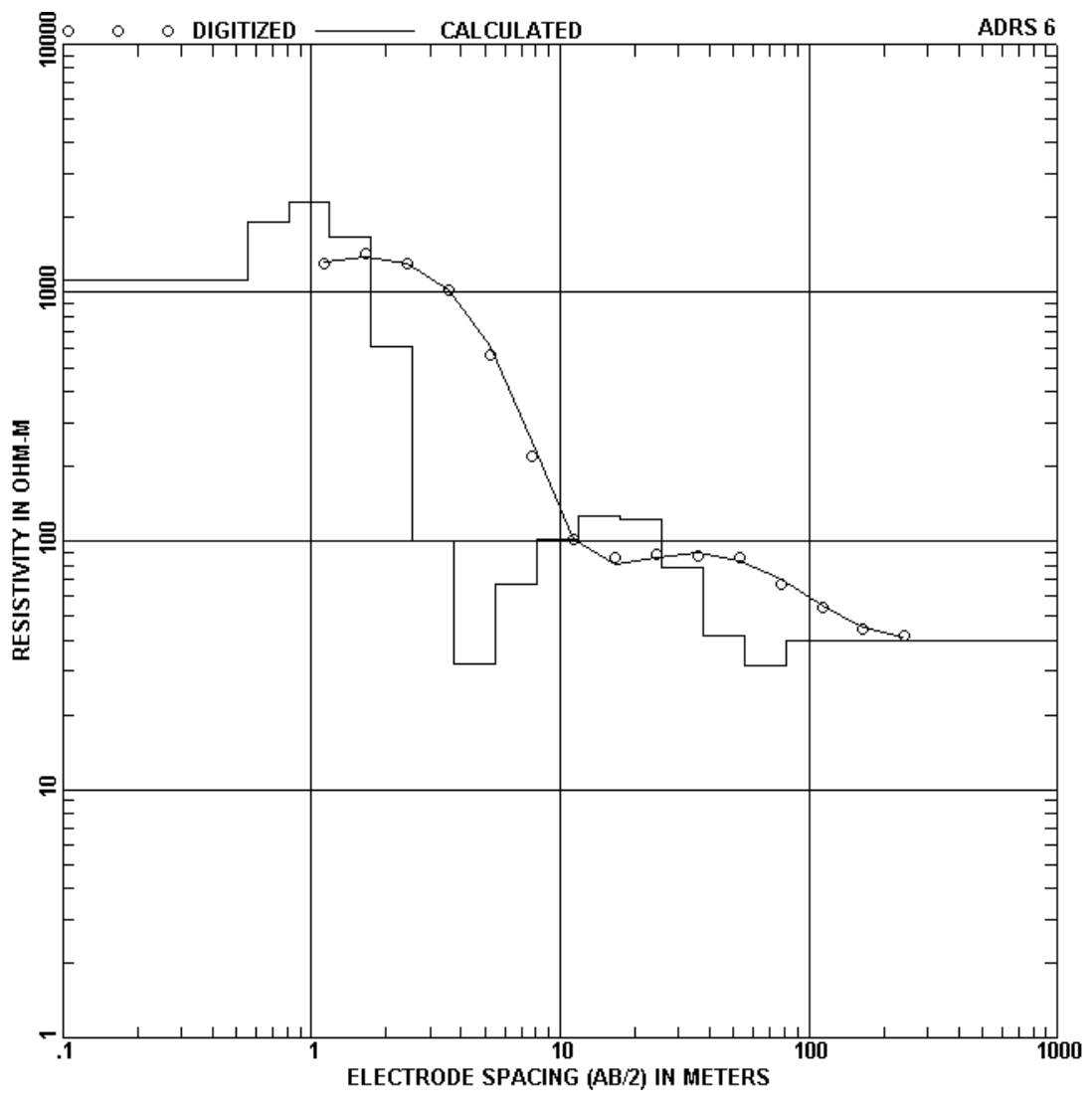

DEPTH, $m$ ( ft ) RESIS. DEPTH, $m$ ( ft ) RESIS.

64.60
65.80
64.20
71.30
71.70
67.00
48.20
58.70
52.30
42.80
41.60

\begin{tabular}{|c|c|c|}
\hline 0.55 & $1.80)$ & 1129.27 \\
\hline 0.81 & $2.65)$ & 1934.75 \\
\hline 1.19 & $3.89)$ & 2302.09 \\
\hline 1.74 & $5.71)$ & 1674.72 \\
\hline 2.55 & $8.38)$ & 603.86 \\
\hline 3.7 & $12.29)$ & 99.42 \\
\hline 5.50 & $18.05)$ & 31.98 \\
\hline
\end{tabular}
$11.85(38.88)$

66.81 102.26 125.95 121.54 78.09 41.58 31.49 39.43

31.98

17.391 $37.47(122.9)$

$55.01(180.47)$ $99999.00(99999.00)$
$8.07(26.49)$

$5.50(18.05)$ 


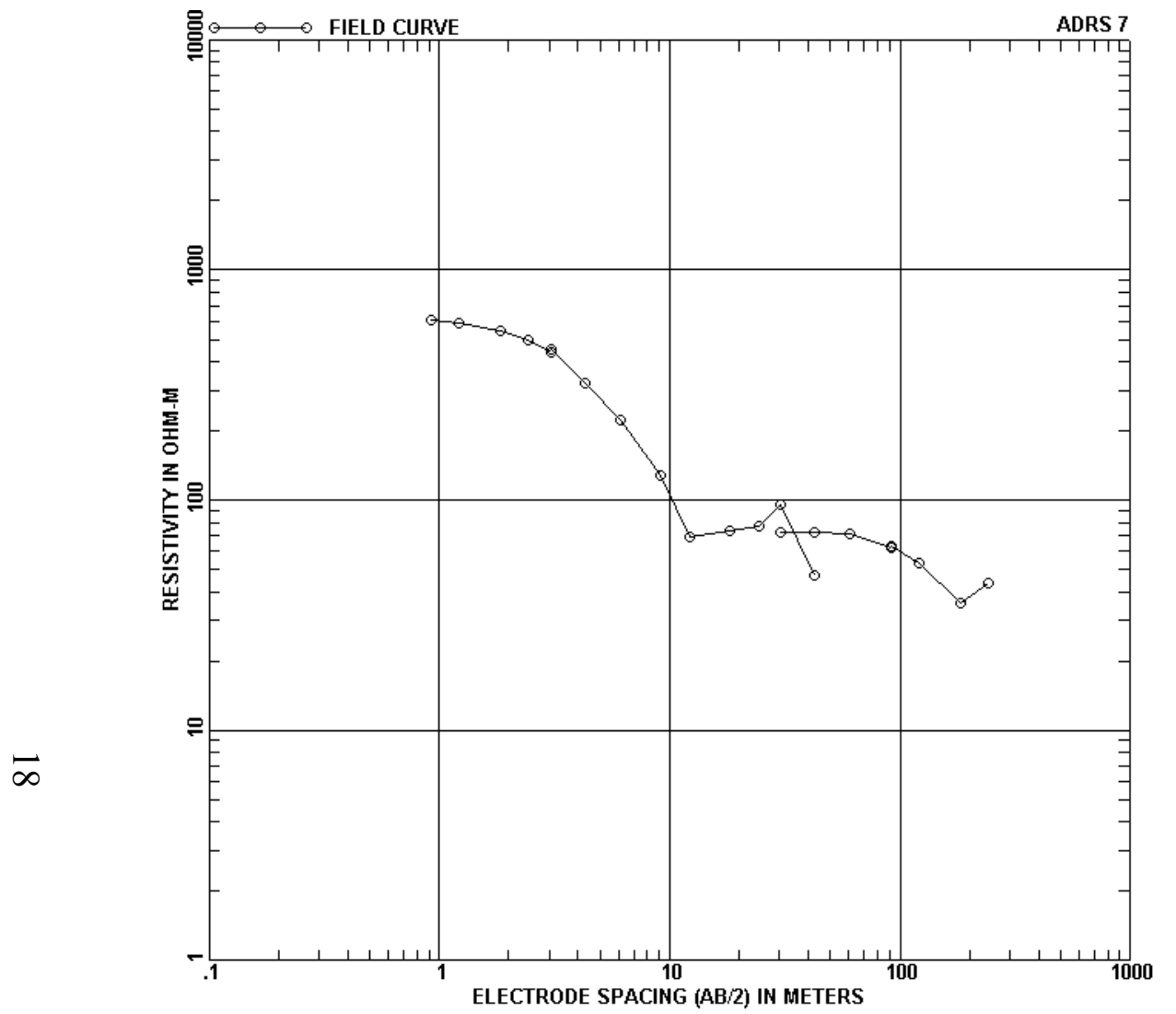

$\mathrm{AB} / 2$, $\mathrm{m}$ ( ft ) App. Res. $\mathrm{AB} / 2$, $\mathrm{m}$ ( ft ) App. Res.

\begin{tabular}{|c|c|c|}
\hline 0.91 & $3.00)$ & 608.00 \\
\hline 1.22 & $4.00)$ & 590.00 \\
\hline 1.83 & $6.00)$ & 546.00 \\
\hline 2.44 & $8.00)$ & 495.00 \\
\hline 3.05 & $10.00)$ & 436.00 \\
\hline 3.05 & $10.00)$ & 450.00 \\
\hline 4.27 & $14.00)$ & 324.00 \\
\hline 6.10 & $20.00)$ & 222.00 \\
\hline 9.14 & $30.00)$ & 128.00 \\
\hline 12.19 & $40.00)$ & 69.00 \\
\hline 18.29 & $60.00)$ & 73.40 \\
\hline
\end{tabular}

76.50
94.90
46.80
72.50
72.80
70.90
61.80
63.40
53.10
35.80
43.50
$121.92(400.00) \quad 53.10$ $243.84(800.00) \quad 43.50$

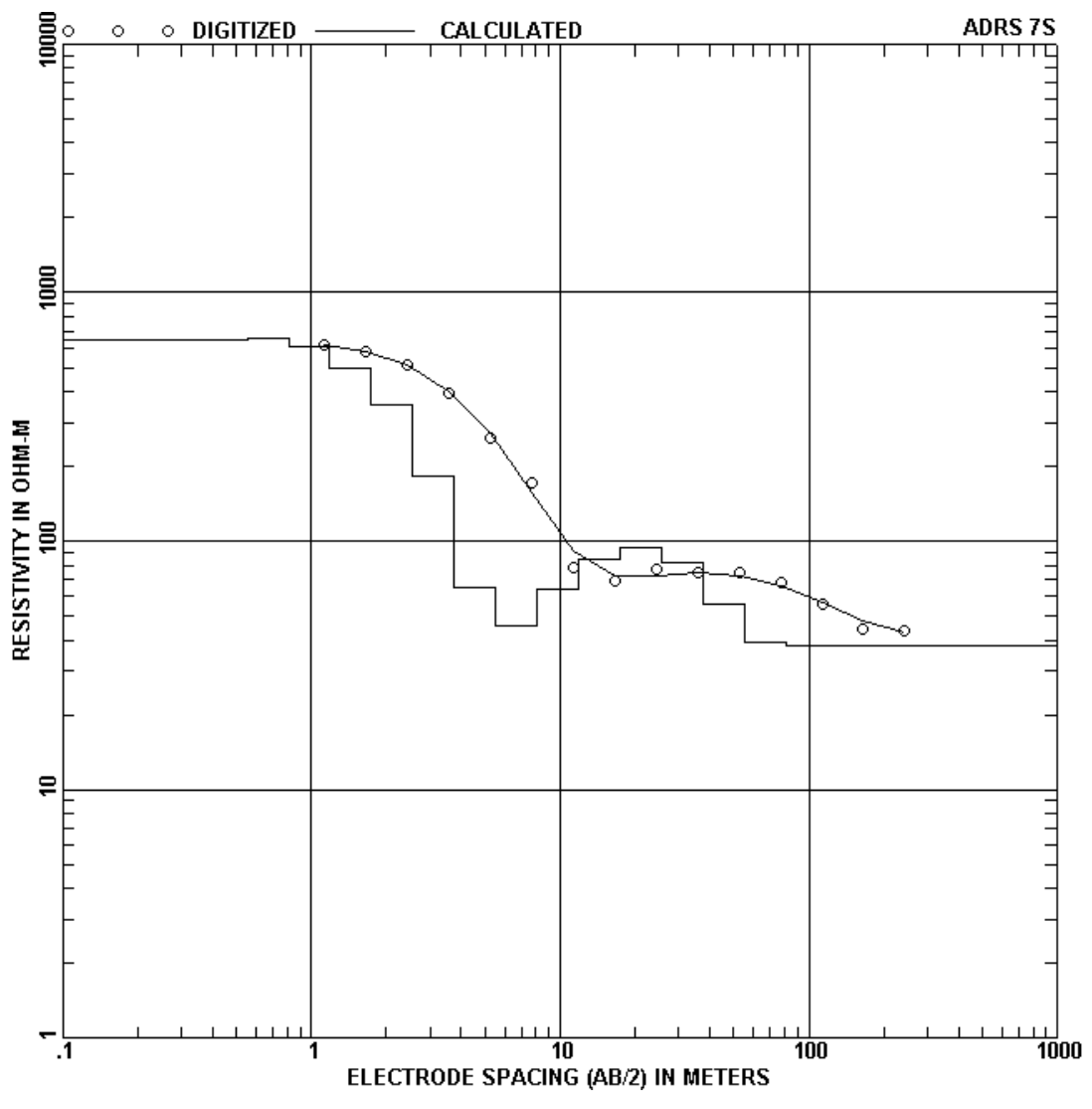

DEPTH, $m$ ( ft ) RESIS. DEPTH, m ( ft ) RESIS.

\begin{tabular}{|c|c|}
\hline 55 & $1.80)$ \\
\hline .0 & $65)$ \\
\hline .1 & $3.89)$ \\
\hline 7 & $5.71)$ \\
\hline .5 & $8.38)$ \\
\hline 7 & $12.29)$ \\
\hline & $18.05)$ \\
\hline
\end{tabular}

639.98

657.29

607.20

494.27

354.49

183.12

64.93
45.76

63.73

84.52

94.60

82.32

56.02

39.32

38.11 


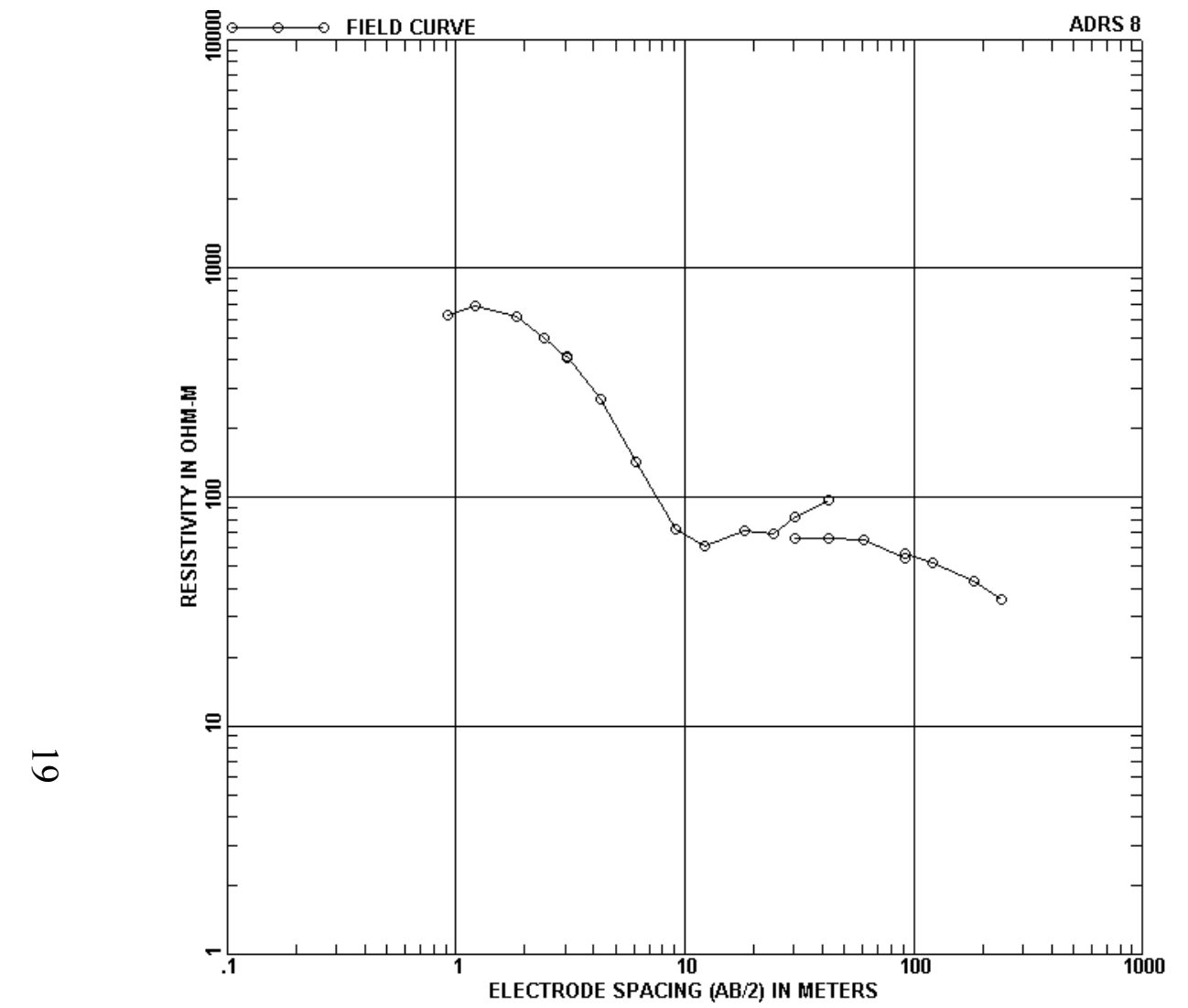

$A B / 2, m$ ( ft ) App. Res. $A B / 2$, m ( ft ) App. Res.

\begin{tabular}{|c|c|c|}
\hline 0.91 & $3.00)$ & 627.00 \\
\hline 1.22 & $4.00)$ & 684.00 \\
\hline 1.83 & $6.00)$ & 611.00 \\
\hline 2.44 & $8.00)$ & 496.00 \\
\hline 3.05 & $10.00)$ & 409.00 \\
\hline 3.05 & $10.00)$ & 411.00 \\
\hline 4.27 & $14.00)$ & 266.00 \\
\hline 6.10 & $20.00)$ & 143.00 \\
\hline 9.14 & $30.00)$ & 72.30 \\
\hline 12.19 & $40.00)$ & 60.80 \\
\hline 18.29 & $60.00)$ & 70.9 \\
\hline
\end{tabular}

69.40
82.00
97.10
66.00
65.80
65.10
54.40
56.50
51.80
42.60
35.70

$121.92(400.00)$

$243.84(800.00)$

35.70

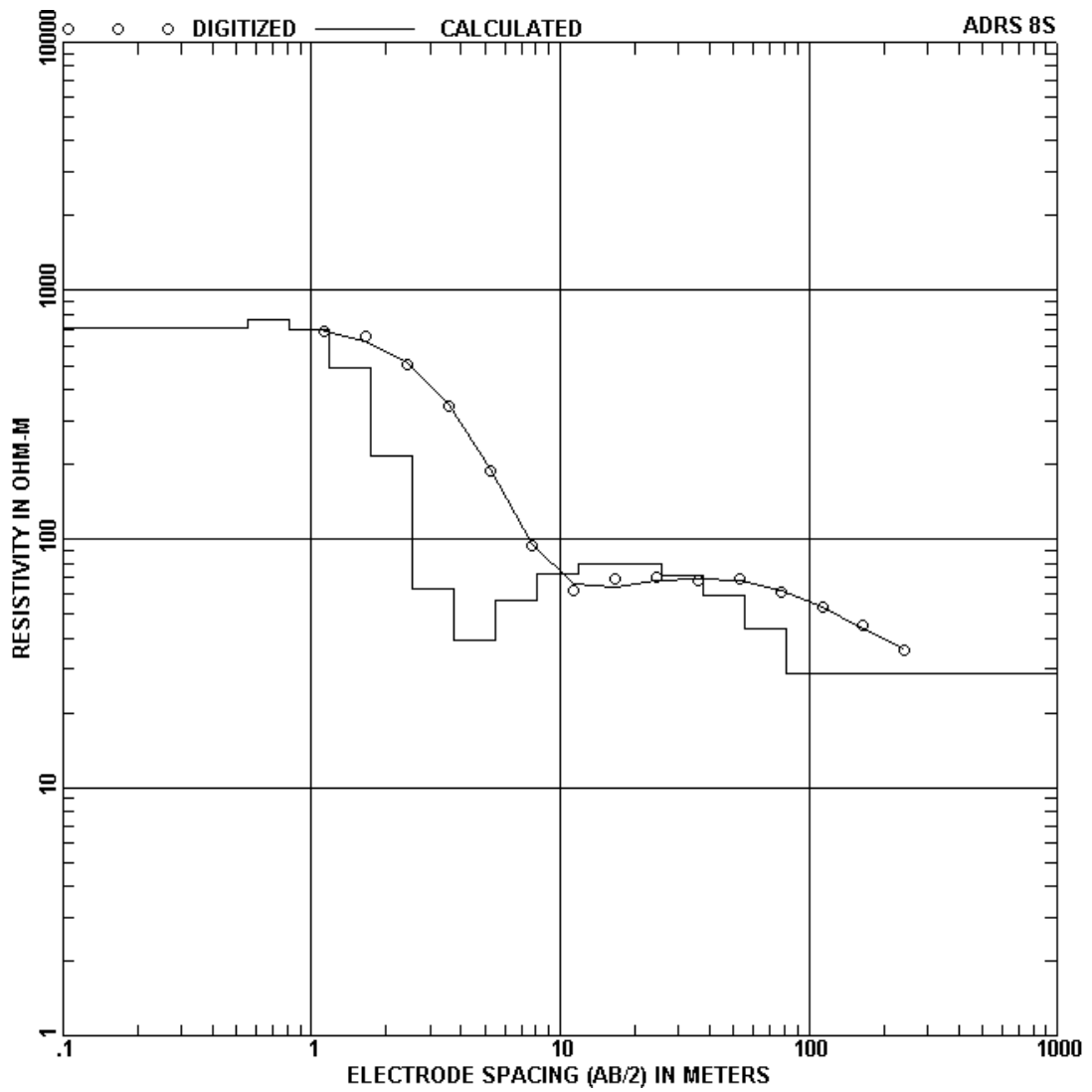

DEPTH, m ( ft ) RESIS. DEPTH, m ( ft ) RESIS.

\begin{tabular}{|c|c|}
\hline 55 & $1.80)$ \\
\hline .0 & $65)$ \\
\hline .1 & $3.89)$ \\
\hline 7 & $5.71)$ \\
\hline .5 & $8.38)$ \\
\hline 7 & $12.29)$ \\
\hline & $18.05)$ \\
\hline
\end{tabular}

710.48

766.90

698.00

487.68

216.52

62.59

38.92
56.22

72.55

79.96

79.16

71.52

59.50

43.49

28.66 


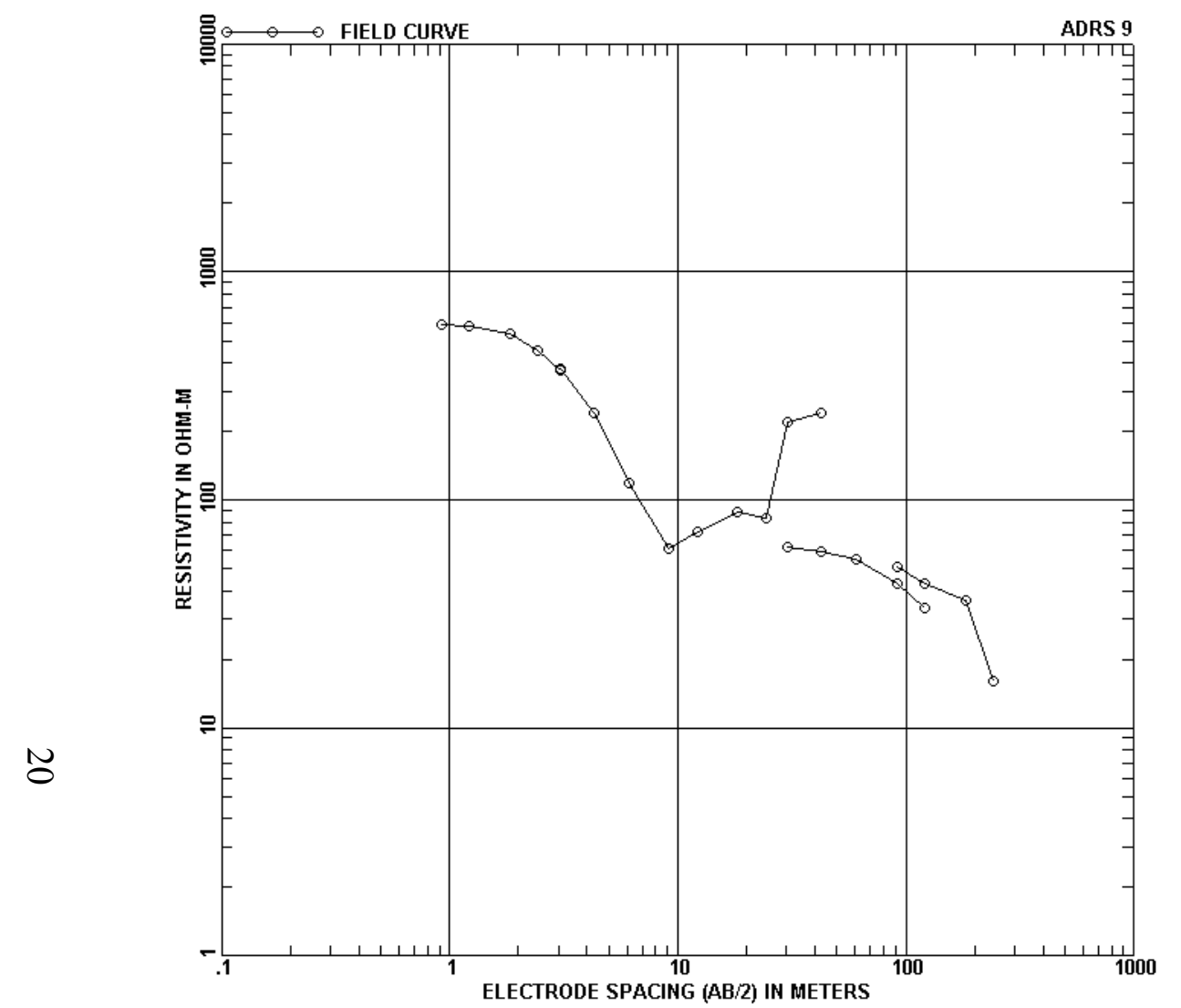

$\mathrm{AB} / 2$, $\mathrm{m}$ ( ft ) App. Res. $\mathrm{AB} / 2$, $\mathrm{m}$ ( ft ) App. Res.

\begin{tabular}{|c|c|c|}
\hline 0.91 & $3.00)$ & 586.00 \\
\hline 1.22 & $4.00)$ & 581.00 \\
\hline 1.83 & $6.00)$ & 540.00 \\
\hline 2.44 & $8.00)$ & 453.00 \\
\hline 3.05 & $10.00)$ & 370.00 \\
\hline 3.05 & $10.00)$ & 377.00 \\
\hline 4.27 & $14.00)$ & 241.00 \\
\hline 6.10 & $20.00)$ & 119.00 \\
\hline 9.14 & $30.00)$ & 61.00 \\
\hline 12.19 & $40.00)$ & 72.20 \\
\hline 18.29 & $60.00)$ & 88.10 \\
\hline
\end{tabular}

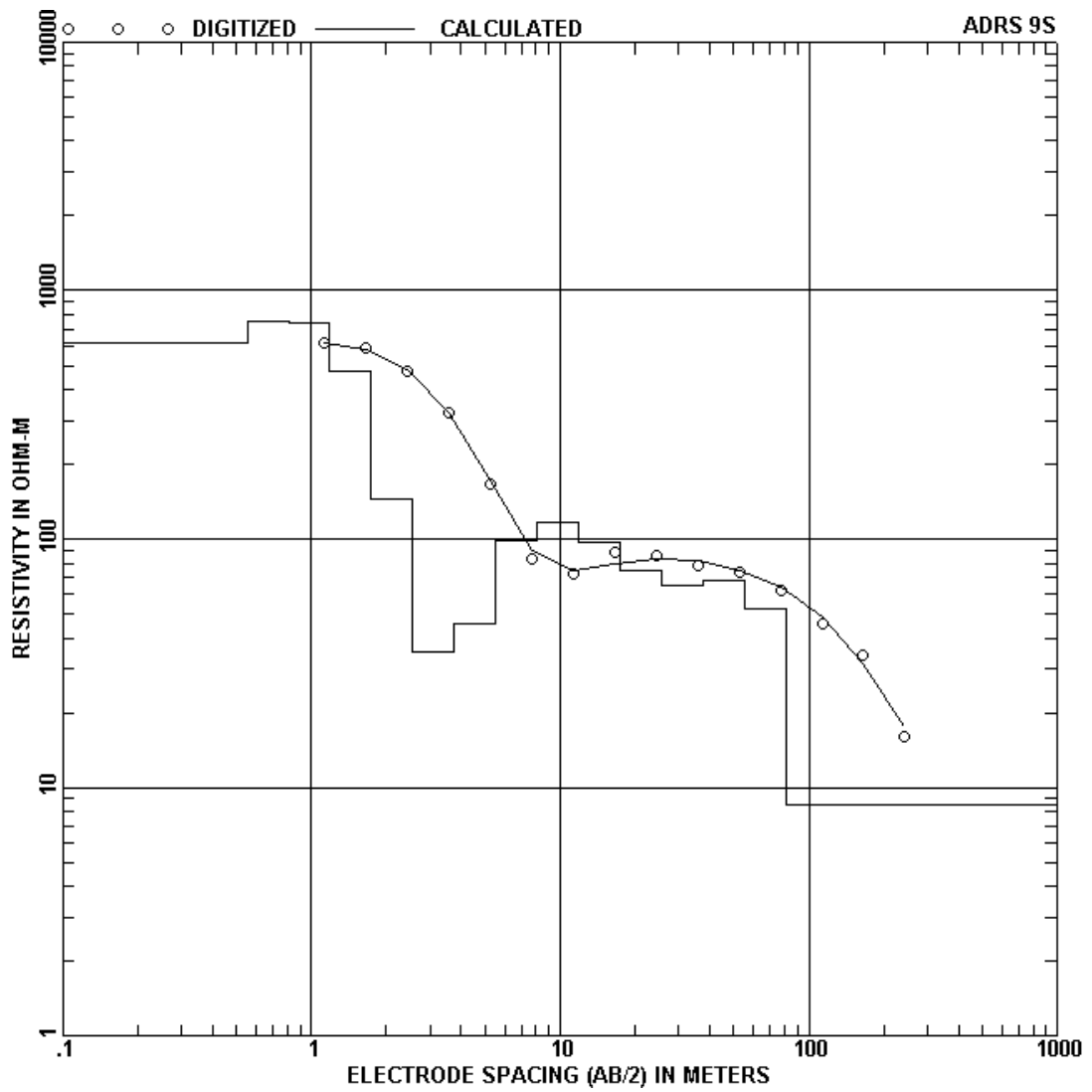

DEPTH, $m$ ( ft ) RESIS. DEPTH, $m$ ( ft ) RESIS.

$\begin{array}{rrr}24.38 & (\quad 80.00) & 83.20 \\ 30.48 & (100.00) & 218.00 \\ 42.67 & (140.00) & 242.00 \\ 30.48 & (100.00) & 62.50 \\ 42.67 & (140.00) & 59.30 \\ 60.96 & (1200.00) & 54.50 \\ 91.44( & (300.00) & 43.00 \\ 121.92( & (400.00) & 33.50 \\ 91.44( & (300.00) & 51.20 \\ 121.92 & (400.00) & 43.10 \\ 182.88 & (600.00) & 36.10 \\ 243.84 & (800.00) & 16.00\end{array}$

$\begin{array}{rlr}0.55 & ( & 1.80) \\ 0.81 & ( & 2.65) \\ 1.19 & ( & 3.89) \\ 1.74 & ( & 5.71) \\ 2.55 & ( & 8.38) \\ 3.75 & ( & 12.29) \\ 5.50 & ( & 18.05)\end{array}$

619.56

749.02

737.69

473.60

144.64

35.12

45.75

\begin{tabular}{|c|c|c|}
\hline 8.07 & $26.49)$ & 97.83 \\
\hline 11.85 & $38.88)$ & 117.38 \\
\hline 17.39 & $57.07)$ & 96.60 \\
\hline 25.53 & $83.76)$ & 74.32 \\
\hline 37.47 & $122.95)$ & 65.33 \\
\hline 55.01 & $180.47)$ & 67.67 \\
\hline 80.74 & $264.89)$ & 52.66 \\
\hline 99.0 & $(99999.00)$ & 8.52 \\
\hline
\end{tabular}




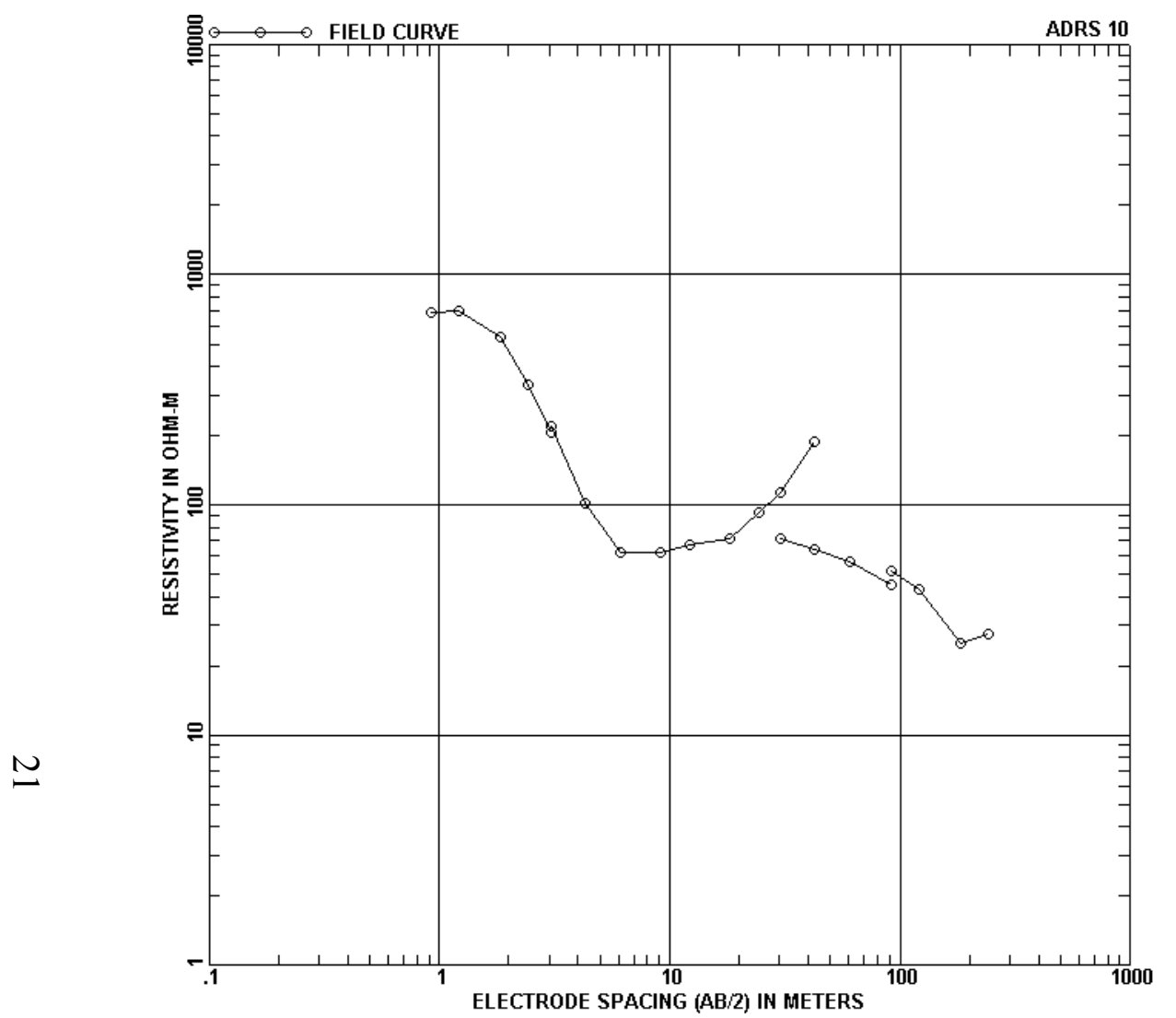

$A B / 2, m$ ( ft ) App. Res. $A B / 2, m$ ( ft ) App. Res.

\begin{tabular}{|c|c|c|}
\hline 0.91 & $3.00)$ & 690.00 \\
\hline 1.22 & $4.00)$ & 699.00 \\
\hline 1.83 & $6.00)$ & 538.00 \\
\hline 2.44 & $8.00)$ & 335.00 \\
\hline 3.05 & $10.00)$ & 207.00 \\
\hline 3.05 & $10.00)$ & 218.00 \\
\hline 4.27 & $14.00)$ & 101.00 \\
\hline 6.10 & $20.00)$ & 61.70 \\
\hline 9.14 & $30.00)$ & 62.50 \\
\hline 2.1 & $40.00)$ & 67.30 \\
\hline 8.29 & $60.00)$ & 71.00 \\
\hline
\end{tabular}

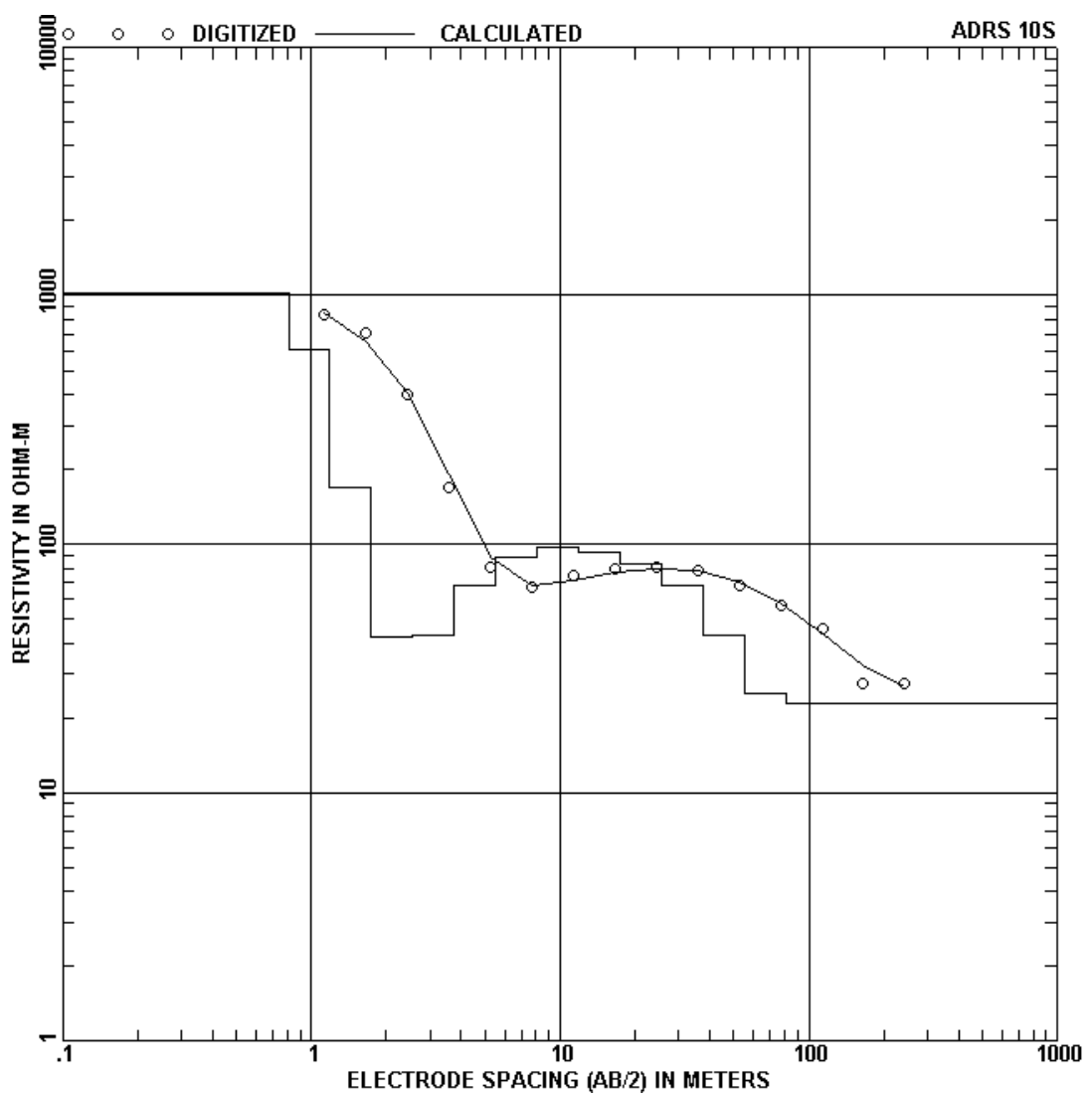

DEPTH, $m$ ( ft ) RESIS. DEPTH, m ( ft ) RESIS.

$\begin{array}{rrrrrr}0.55( & 1.80) & 1015.95 & 8.07( & 26.49) & 88.20 \\ 0.81( & 2.65) & 1020.61 & 11.85( & 38.88) & 96.36 \\ 1.19( & 3.89) & 601.78 & 17.39( & 57.07) & 93.18 \\ 1.74( & 5.71) & 168.41 & 25.53( & 83.76) & 83.65 \\ 2.55( & 8.38) & 42.01 & 37.47(122.95) & 67.94 \\ 3.75( & 12.29) & 42.76 & 55.01(180.47) & 43.19 \\ 5.50( & 18.05) & 67.91 & 80.74(264.89) & 24.92 \\ & & & 99999.00 & (99999.00) & 22.98\end{array}$




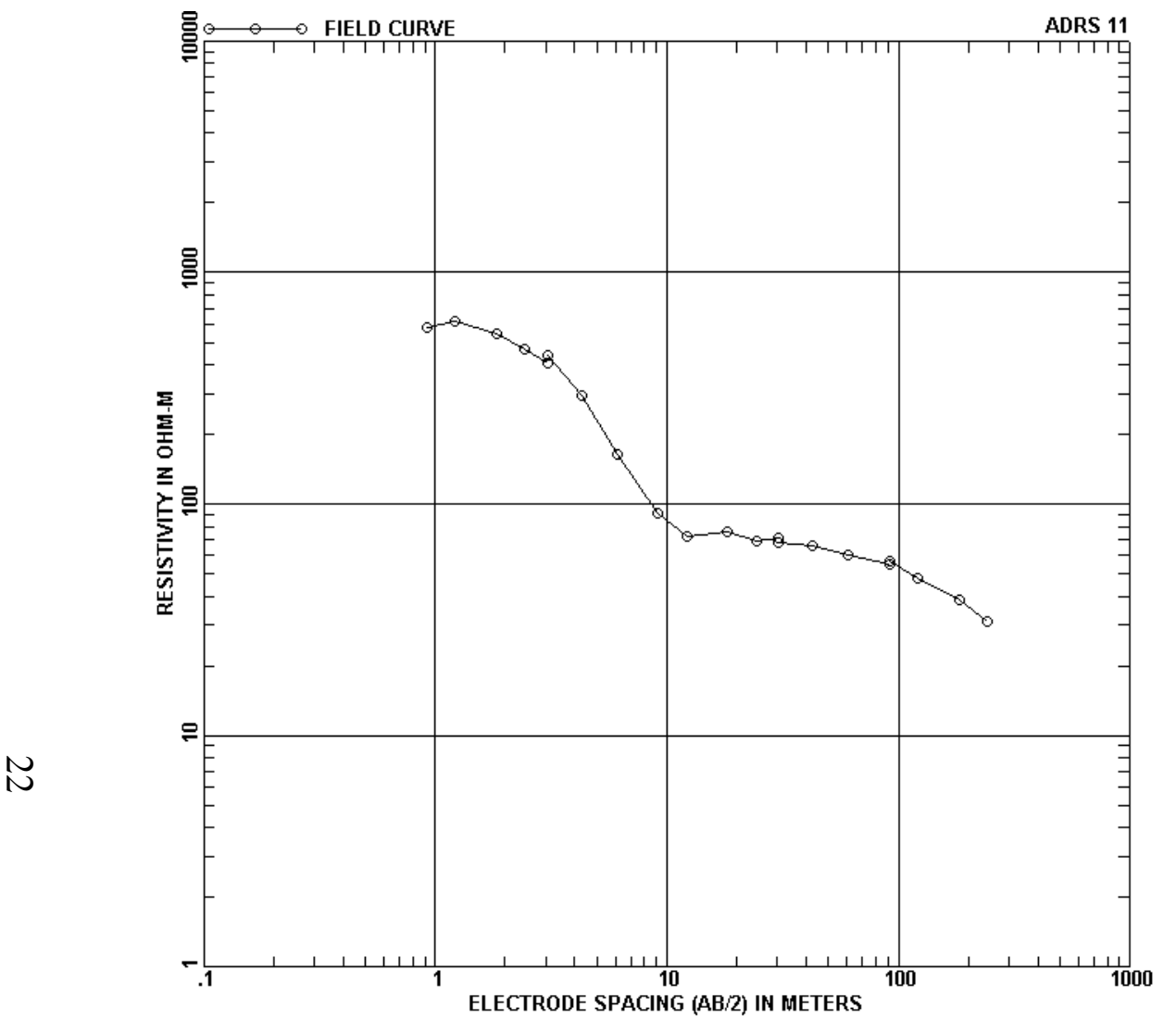

$A B / 2, m(f t)$ App. Res. $A B / 2, m$ ( ft ) App. Res.

\begin{tabular}{|c|c|c|}
\hline 0.91 & $3.00)$ & 578.00 \\
\hline 1.22 & $4.00)$ & 620.00 \\
\hline 1.83 & $6.00)$ & 541.00 \\
\hline 2.44 & $8.00)$ & 469.00 \\
\hline 3.05 & $10.00)$ & 409.00 \\
\hline 3.05 & $10.00)$ & 441.00 \\
\hline 4.27 & $14.00)$ & 292.00 \\
\hline 6.10 & $20.00)$ & 164.00 \\
\hline 9.14 & $30.00)$ & 90.90 \\
\hline 12.19 & $40.00)$ & 72.80 \\
\hline
\end{tabular}

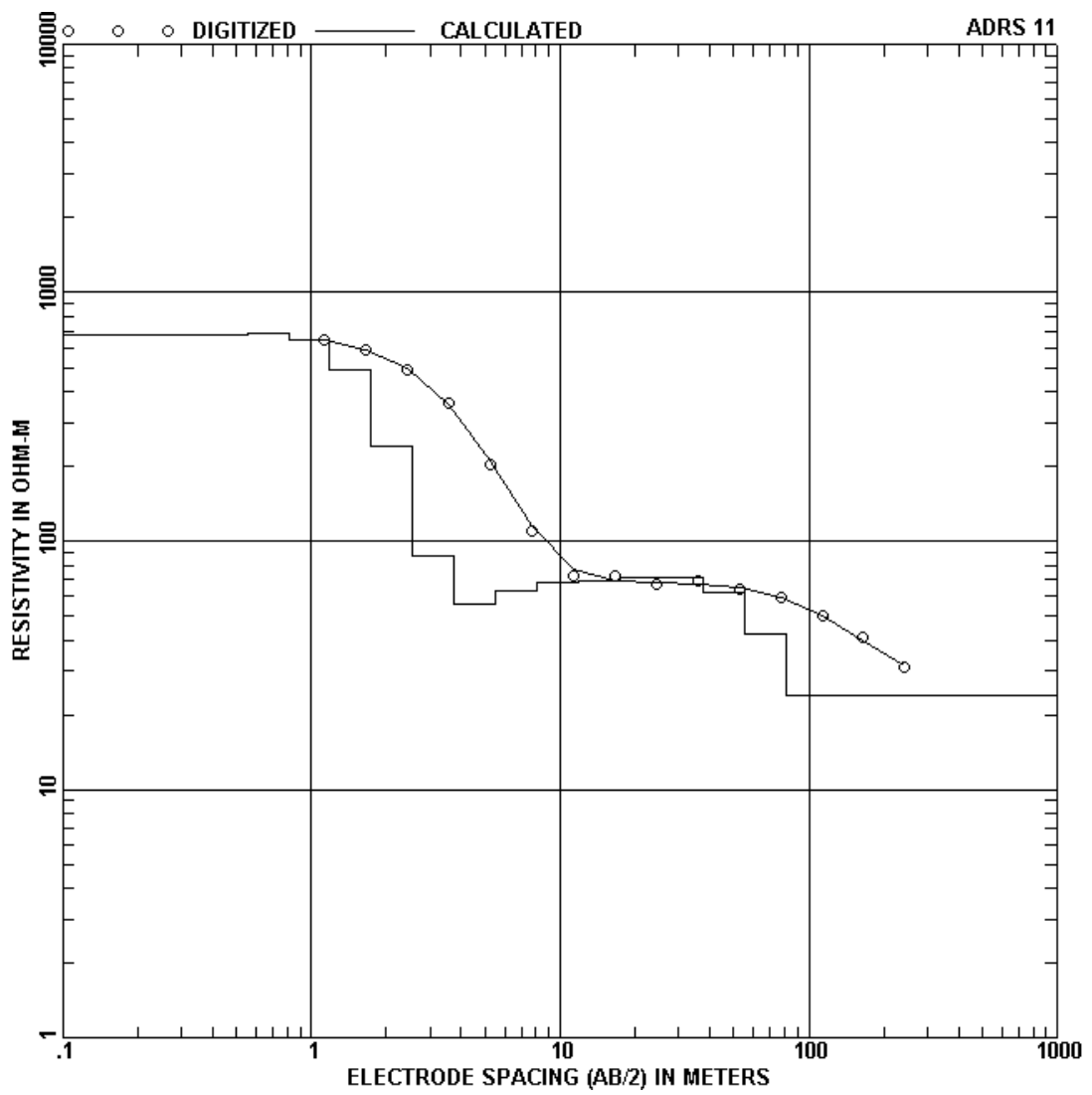

DEPTH, m ( ft ) RESIS. DEPTH, m ( ft ) RESIS.

75.70
69.30
71.70
67.90
66.00
60.60
55.20
56.20
48.00
38.50
31.00

\begin{tabular}{|c|c|}
\hline 55 & $1.80)$ \\
\hline .0 & $65)$ \\
\hline .1 & $3.89)$ \\
\hline 7 & $5.71)$ \\
\hline .5 & $8.38)$ \\
\hline 7 & $12.29)$ \\
\hline & $18.05)$ \\
\hline
\end{tabular}

678.23

685.34

645.88

487.54

240.74

87.28

55.45
62.90

67.77

69.31

71.21

71.20

61.78

42.01

24.07

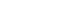




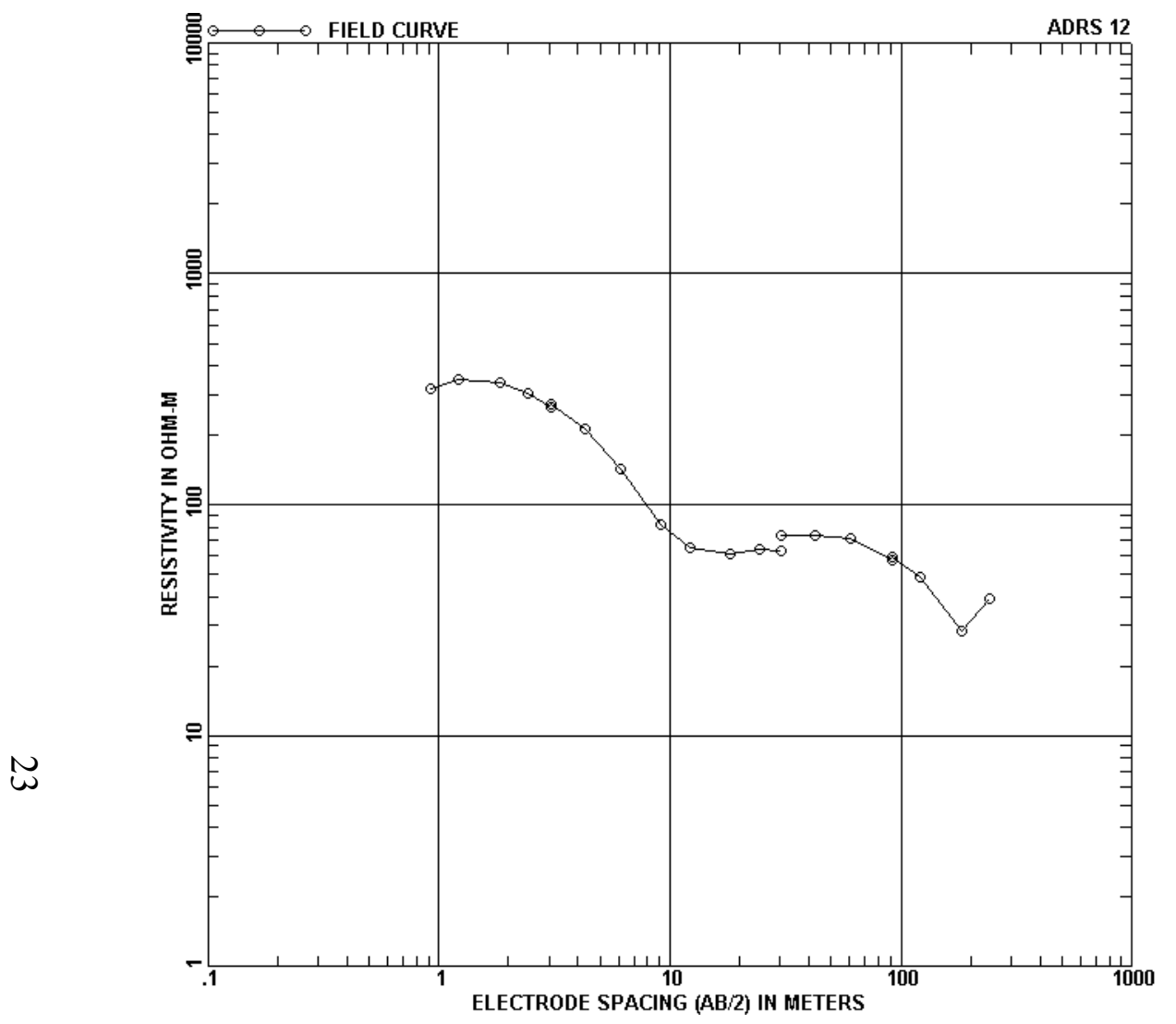

$A B / 2, m$ ( ft ) App. Res. $A B / 2, m$ ( ft ) App. Res.

\begin{tabular}{|c|c|c|}
\hline 0.91 & $3.00)$ & 316.00 \\
\hline 1.22 & $4.00)$ & 350.00 \\
\hline 1.83 & $6.00)$ & 337.00 \\
\hline 2.44 & $8.00)$ & 302.00 \\
\hline 3.05 & $10.00)$ & 264.00 \\
\hline 3.05 & $10.00)$ & 273.00 \\
\hline 4.27 & $14.00)$ & 213.00 \\
\hline 6.10 & $20.00)$ & 142.00 \\
\hline 9.14 & $30.00)$ & 82.20 \\
\hline 12.19 & $40.00)$ & 65.50 \\
\hline
\end{tabular}

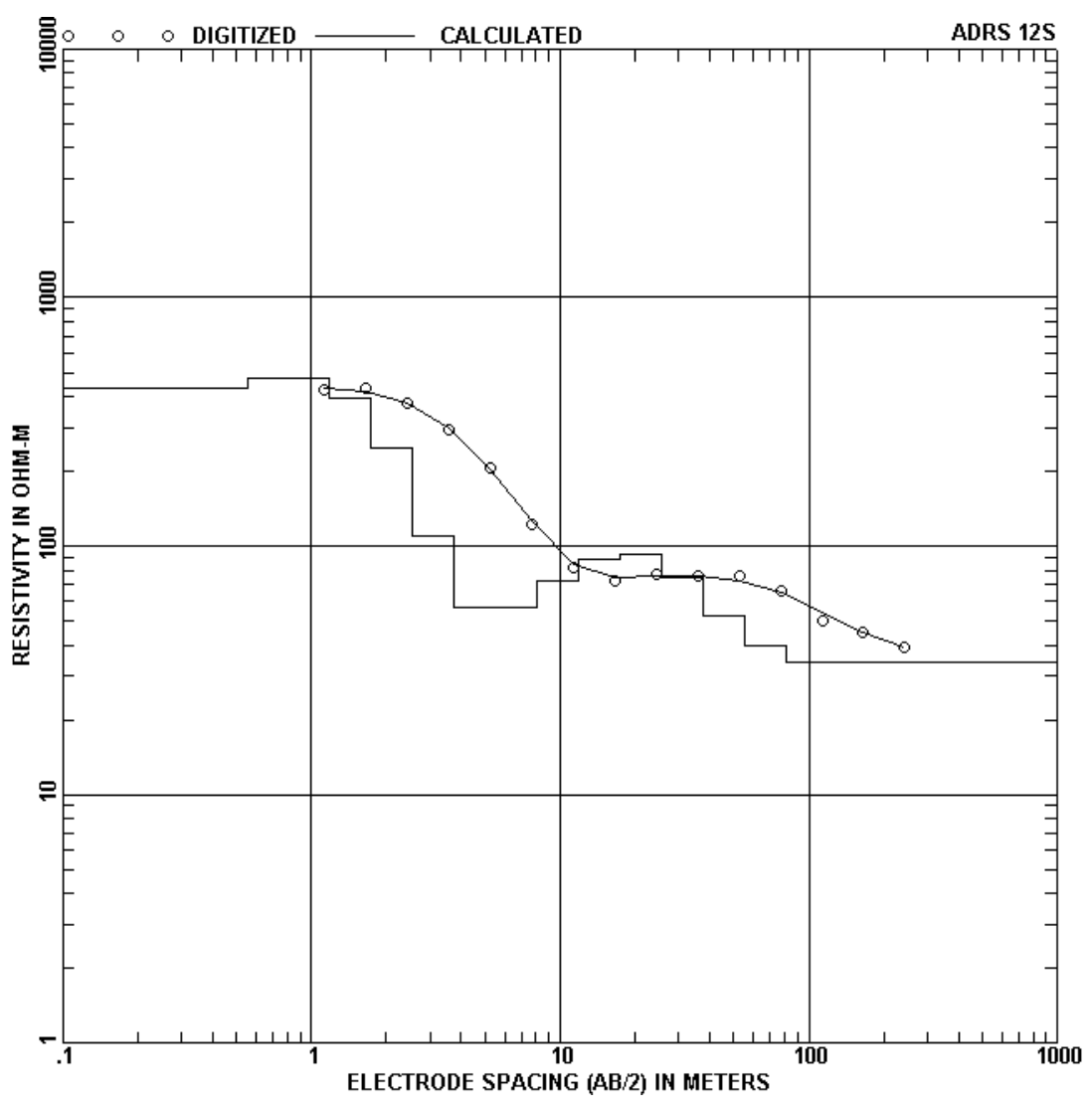

DEPTH, m ( ft ) RESIS. DEPTH, m ( ft ) RESIS.

60.80
64.30
62.90
73.40
74.10
71.10
57.20
59.00
48.20
28.40
39.30

\begin{tabular}{|c|c|}
\hline 55 & $1.80)$ \\
\hline .0 & $65)$ \\
\hline .1 & $3.89)$ \\
\hline 7 & $5.71)$ \\
\hline .5 & $8.38)$ \\
\hline 7 & $12.29)$ \\
\hline & $18.05)$ \\
\hline
\end{tabular}

433.81

471.29

471.28

396.90

246.86

109.89

56.97

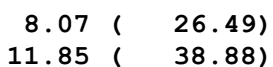

$\begin{array}{ll}11.85 & (38.88) \\ 17.39 & (\quad 57.07)\end{array}$

56.43

72.58

89.00

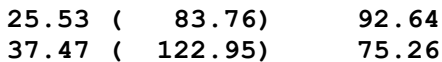
$55.01(180.47) \quad 52.53$

$80.74(264.89) \quad 39.60$ $99999.00(99999.00) \quad 34.22$ 


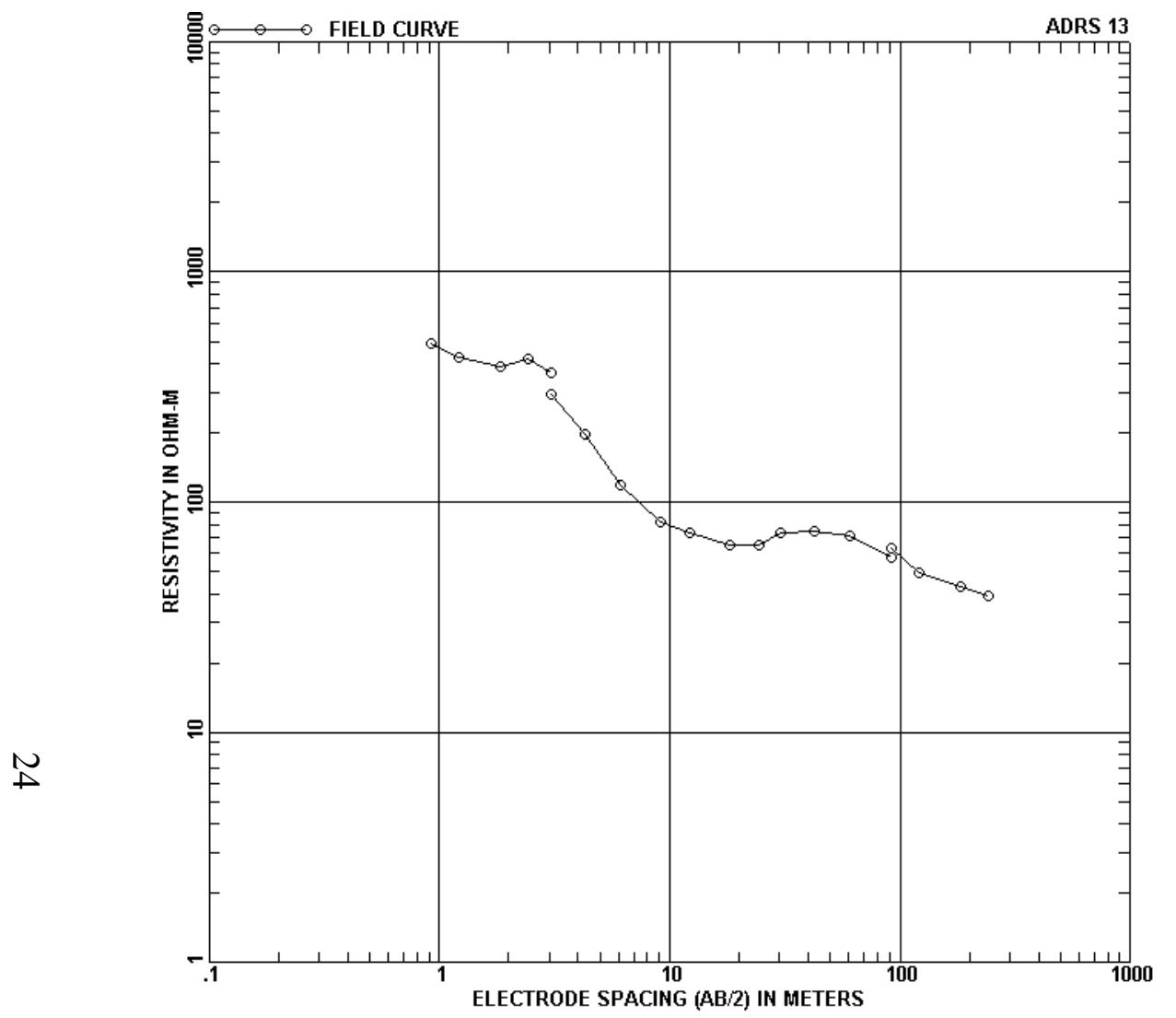

$A B / 2, m(f t)$ App. Res. $A B / 2, m$ ( ft ) App. Res.

\begin{tabular}{|c|c|c|}
\hline 0.91 & $3.00)$ & 489.00 \\
\hline 1.22 & $4.00)$ & 424.00 \\
\hline 1.83 & $6.00)$ & 389.00 \\
\hline 2.44 & $8.00)$ & 422.00 \\
\hline 3.05 & $10.00)$ & 363.00 \\
\hline 3.05 & $10.00)$ & 292.00 \\
\hline 4.27 & $14.00)$ & 198.00 \\
\hline 6.10 & $20.00)$ & 119.00 \\
\hline 9.14 & $30.00)$ & 82.00 \\
\hline 12.19 & $40.00)$ & 74.10 \\
\hline
\end{tabular}

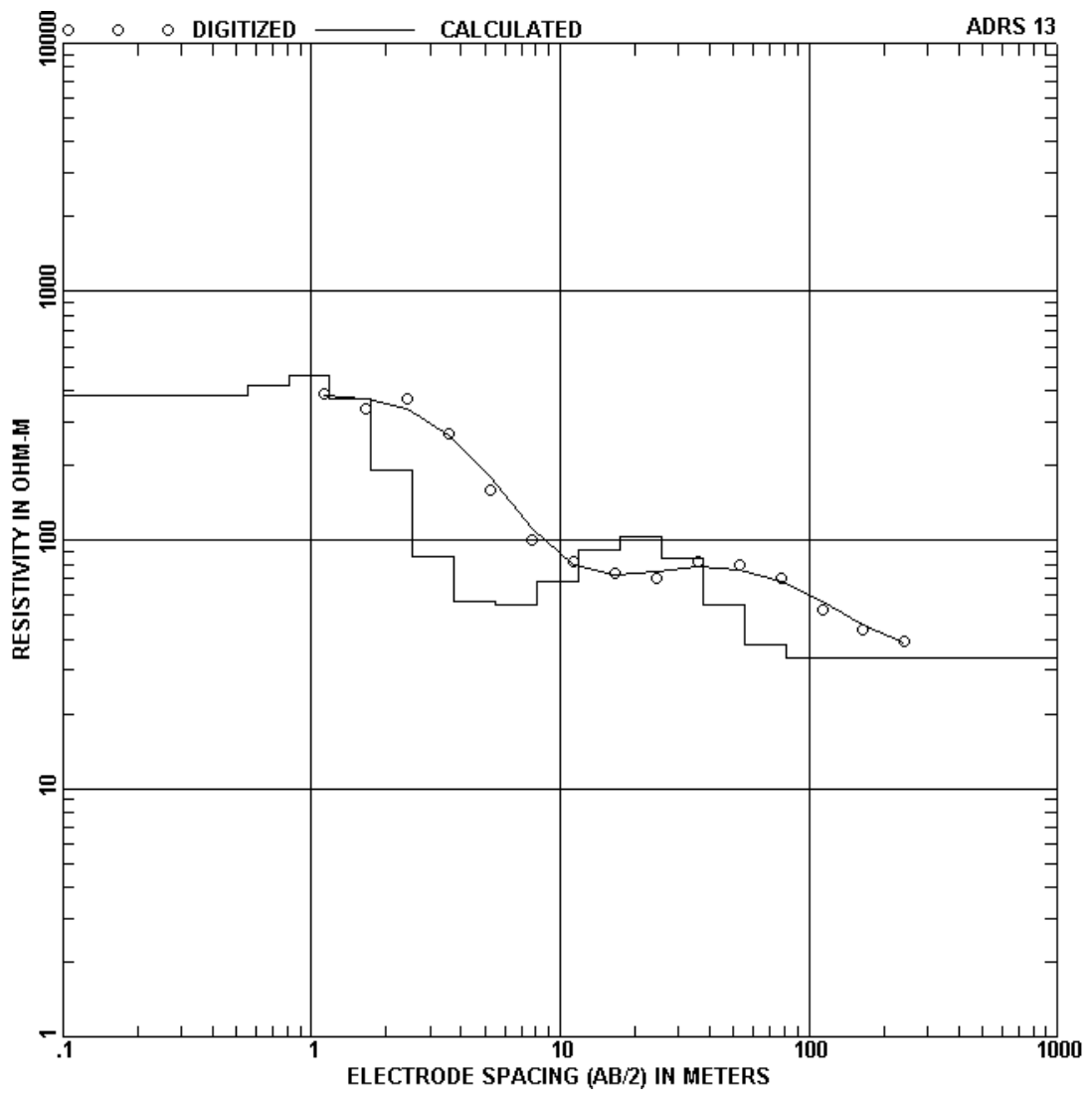

DEPTH, m ( ft ) RESIS. DEPTH, m ( ft ) RESIS.

65.20
64.70
73.10
73.40
75.20
71.40
57.80
62.70
49.50
42.70
39.20

\begin{tabular}{|c|c|}
\hline 55 & $1.80)$ \\
\hline .0 & $65)$ \\
\hline .1 & $3.89)$ \\
\hline 7 & $5.71)$ \\
\hline .5 & $8.38)$ \\
\hline 7 & $12.29)$ \\
\hline & $18.05)$ \\
\hline
\end{tabular}

380.67

416.17

456.31

368.39

191.12

85.67

56.85
55.04

68.54

91.85

102.52

84.40

54.99

37.70

33.38
39.20
$11.85 \div \quad 38$.

17.39 (

$55.01(180.4$

$99999.00 \quad(9999$ 


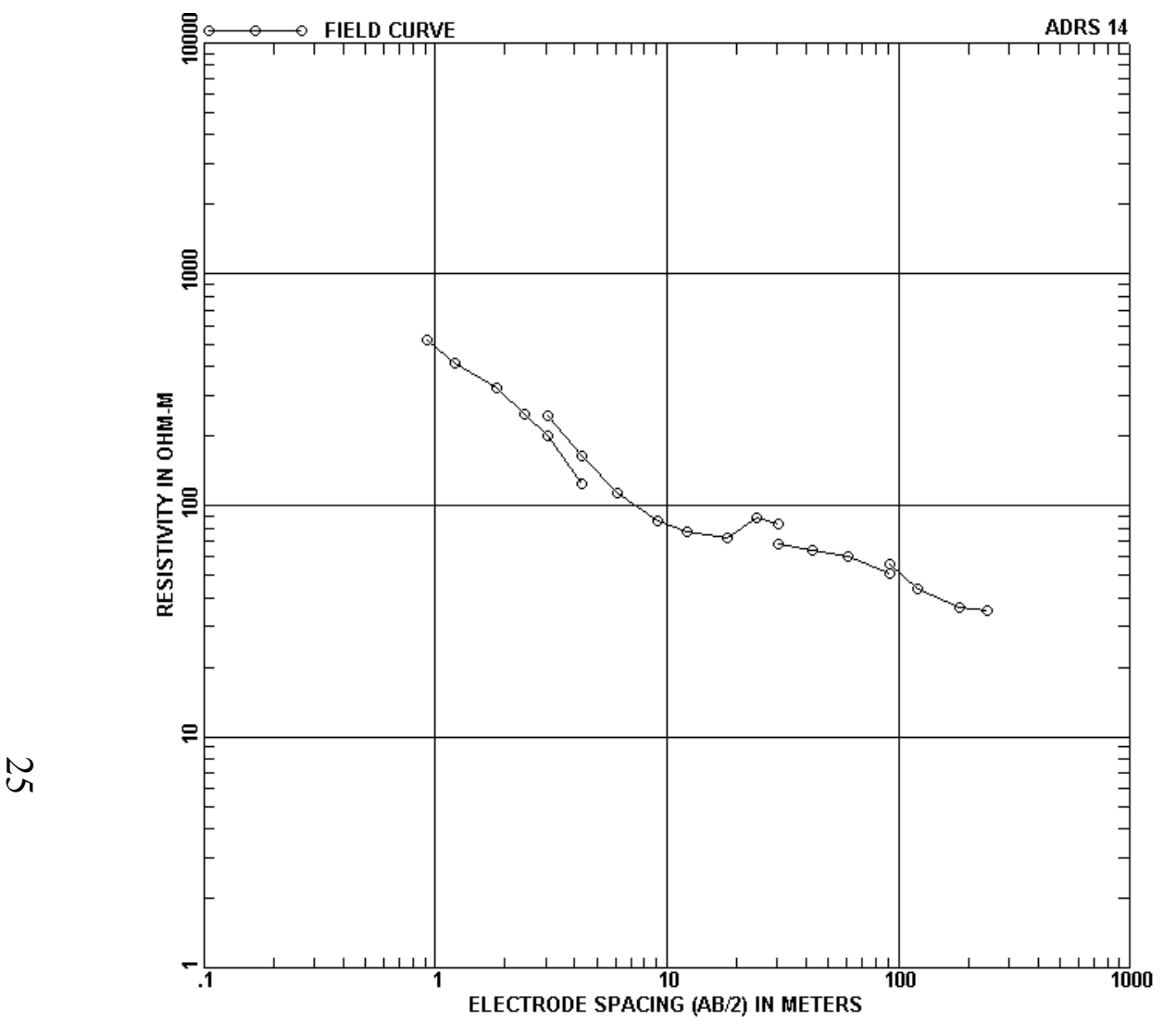

$A B / 2, m$ ( ft ) App. Res. $A B / 2$, m ( ft ) App. Res.

\begin{tabular}{|c|c|c|}
\hline 0.91 & $3.00)$ & 516.00 \\
\hline 1.22 & $4.00)$ & 414.00 \\
\hline 1.83 & $6.00)$ & 323.00 \\
\hline 2.44 & $8.00)$ & 249.00 \\
\hline 3.05 & $10.00)$ & 200.00 \\
\hline 4.27 & $14.00)$ & 124.00 \\
\hline 3.05 & $10.00)$ & 245.00 \\
\hline 4.27 & $14.00)$ & 163.00 \\
\hline 6.10 & $20.00)$ & 113.00 \\
\hline 9.14 & $30.00)$ & 86.40 \\
\hline 2.19 & $40.00)$ & 76.80 \\
\hline
\end{tabular}

\begin{tabular}{|c|c|}
\hline 29 & 0) \\
\hline 24. & \\
\hline .2 & 100 \\
\hline & 10 \\
\hline . & 140 \\
\hline.$\varsigma$ & 200 \\
\hline 91.44 & 300 \\
\hline 91.44 & 300 \\
\hline 1 & 0) \\
\hline 28 & 600 \\
\hline 43.8 & 800.00 \\
\hline
\end{tabular}

72.70
88.00
83.20
67.90
64.50
60.60
51.10
55.50
43.90
36.10
35.10

$182.88(600.00)$

35.10

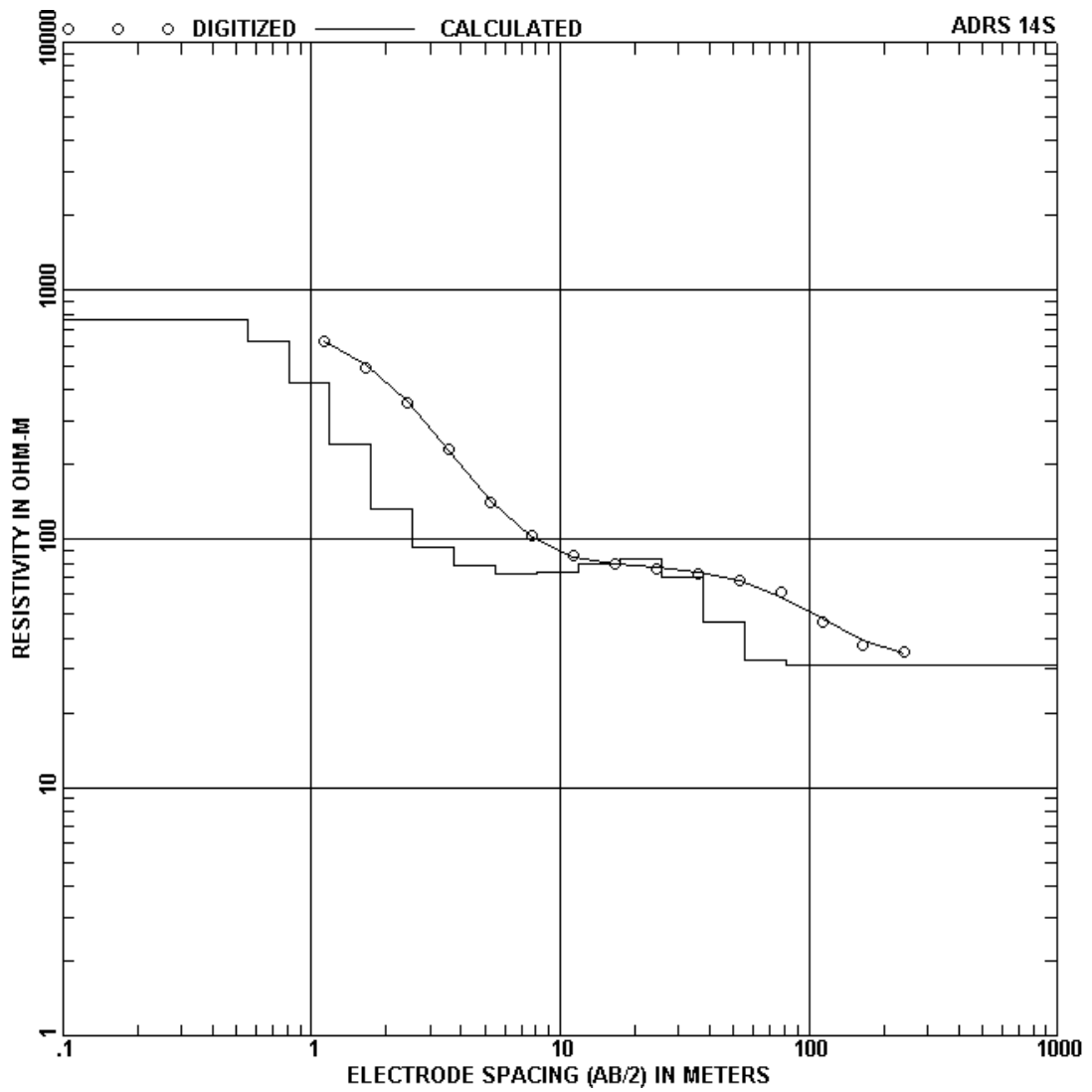

DEPTH, m ( ft ) RESIS. DEPTH, m ( ft ) RESIS.

\begin{tabular}{|c|c|}
\hline 55 & $1.80)$ \\
\hline .0 & $65)$ \\
\hline .1 & $3.89)$ \\
\hline 7 & $5.71)$ \\
\hline .5 & $8.38)$ \\
\hline 7 & $12.29)$ \\
\hline & $18.05)$ \\
\hline
\end{tabular}

765.68
622.75

622.75

423.58

239.99

132.88

92.59

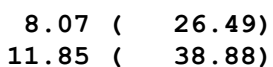

$\begin{array}{ll}11.85 & (38.88) \\ 17.39 & (\quad 57.07)\end{array}$

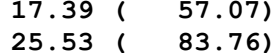

$37.47(122.95)$

$55.01(180.47)$

$80.74(264.89)$

$99999.00 \quad(99999.00)$
72.58

73.29

79.46

83.41

70.71

46.67

32.32

30.88 


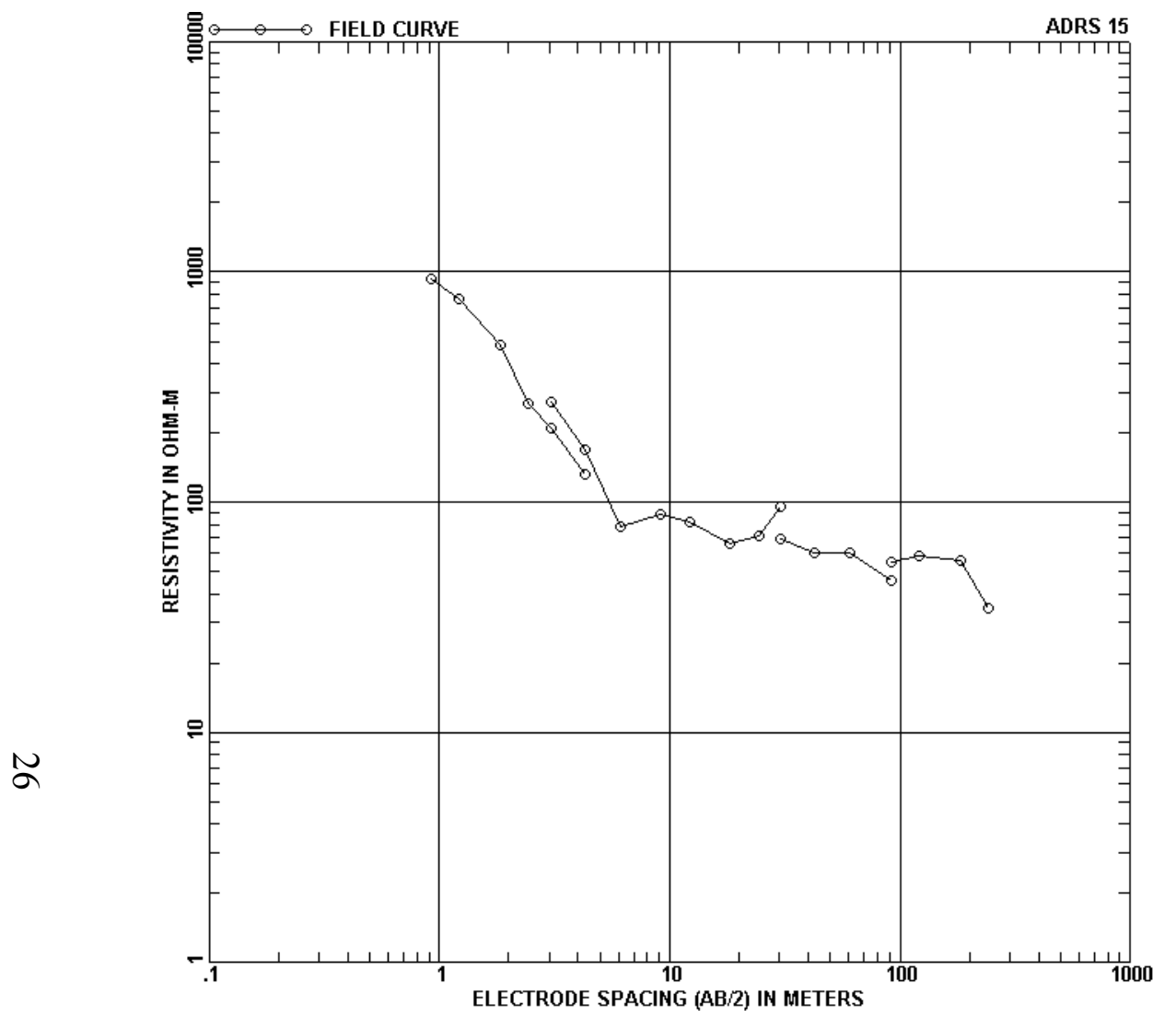

$A B / 2, m(f t)$ App. Res. $A B / 2, m$ ( ft ) App. Res.

\begin{tabular}{|c|c|c|}
\hline 0.91 & $3.00)$ & 940.00 \\
\hline 1.22 & $4.00)$ & 766.00 \\
\hline 1.83 & $6.00)$ & 479.00 \\
\hline 2.44 & $8.00)$ & 270.00 \\
\hline 3.05 & $10.00)$ & 211.00 \\
\hline 4.27 & $14.00)$ & 132.00 \\
\hline 3.05 & $10.00)$ & 272.00 \\
\hline 4.27 & $14.00)$ & 168.00 \\
\hline 6.10 & $20.00)$ & 78.80 \\
\hline 9.14 & $30.00)$ & 88.20 \\
\hline 2.19 & $40.00)$ & 81.30 \\
\hline
\end{tabular}

65.80
71.30
95.60
68.80
60.60
59.90
45.80
55.30
58.20
55.70
34.50
$182.88(600.00)$ $243.84(800.00)$

34.50

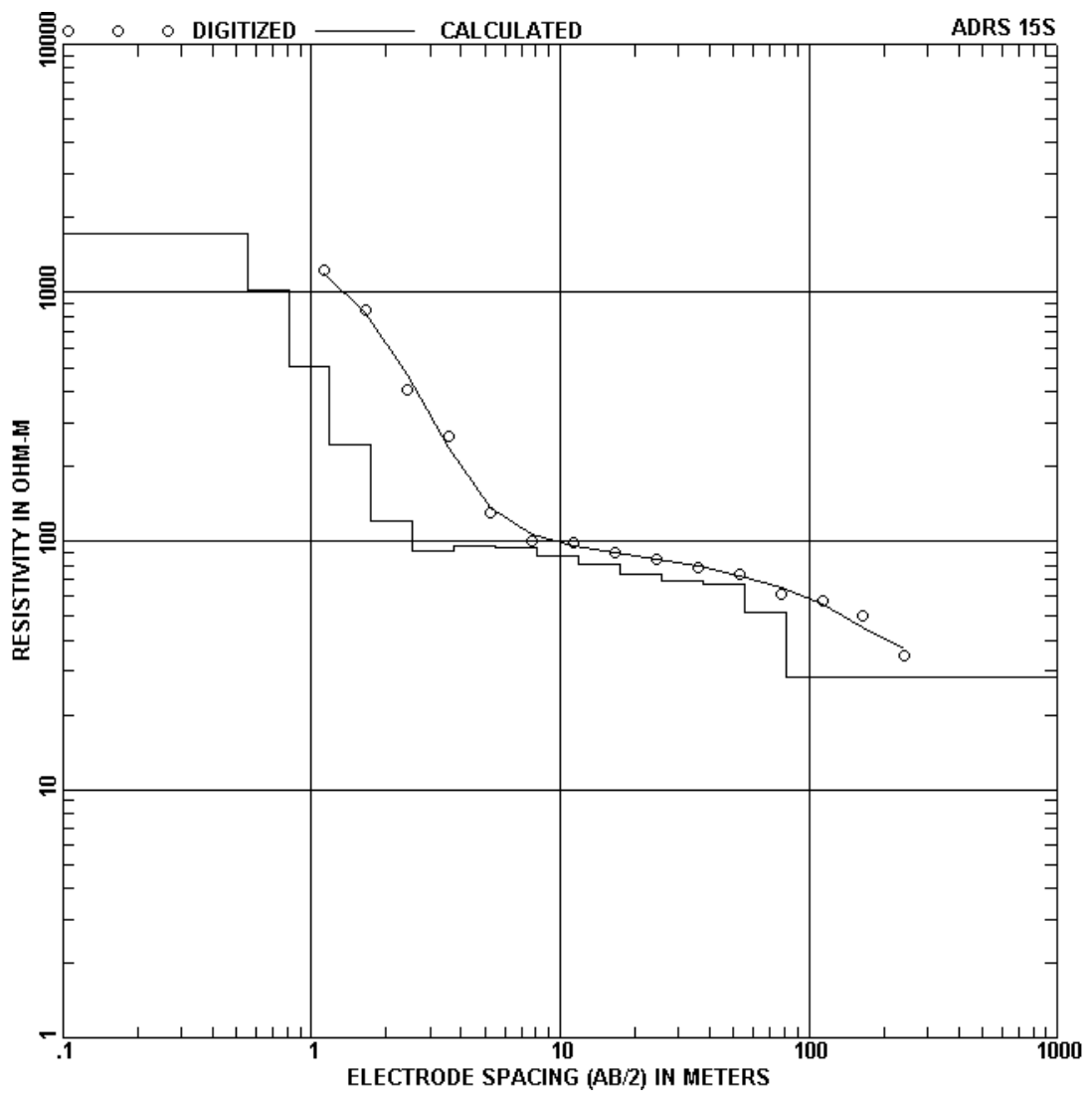

DEPTH, $m$ ( ft ) RESIS. DEPTH, m ( ft ) RESIS.

$\begin{array}{rrr}0.55( & 1.80) & 1730.67 \\ 0.81( & 2.65) & 1029.10 \\ 1.19( & 3.89) & 507.58 \\ 1.74( & 5.71) & 242.92 \\ 2.55( & 8.38) & 121.20 \\ 3.75( & 12.29) & 91.67 \\ 5.50( & 18.05) & 95.09\end{array}$

$8.07(26.49)$ $11.85(38.88)$
$17.39(57.07)$

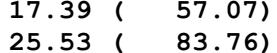
$37.47(122.95)$ 55.01 ( 180.47) $80.74(264.89)$ $99999.00 \quad(99999.00)$
93.79 87.67 80.71 73.39 69.42 66.70 51.37 28.42 

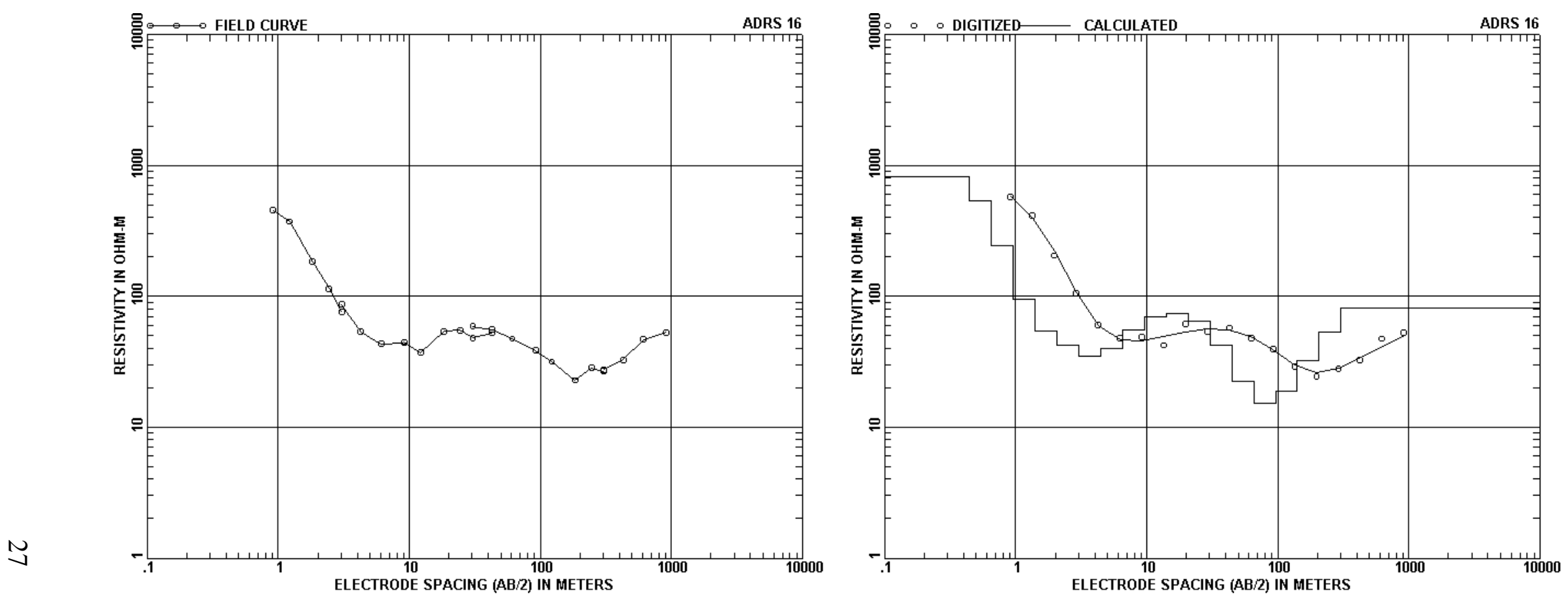

$A B / 2, m$ ( ft ) App. Res. $A B / 2$, m ( ft ) App. Res.

DEPTH, $m$ ( ft ) RESIS. DEPTH, m ( ft ) RESIS.

\begin{tabular}{|c|c|c|c|c|c|}
\hline 0.91 & $3.00)$ & 453.00 & 42.67 & $140.00)$ & 52.40 \\
\hline 1.22 & $4.00)$ & 372.00 & 30.48 & $100.00)$ & 58.90 \\
\hline 1.83 & $6.00)$ & 183.00 & 42.67 & $140.00)$ & 55.80 \\
\hline 2.44 & $8.00)$ & 114.00 & 60.96 & $200.00)$ & 47.40 \\
\hline 3.05 & $10.00)$ & 75.90 & 91.44 & $300.00)$ & 38.60 \\
\hline 3.05 & $10.00)$ & 86.70 & 91.44 & $300.00)$ & 38.60 \\
\hline 4.27 & $14.00)$ & 53.70 & 121.92 & $400.00)$ & 31.60 \\
\hline 6.10 & $20.00)$ & 43.30 & 182.88 & $600.00)$ & 22.80 \\
\hline 9.14 & $30.00)$ & 44.30 & 243.84 & $800.00)$ & 28.30 \\
\hline 9.14 & $30.00)$ & 44.80 & 304.80 & $(1000.00)$ & 26.70 \\
\hline 12.19 & $40.00)$ & 37.10 & 304.80 & $(1000.00)$ & 27.50 \\
\hline 18.29 & $60.00)$ & 54.00 & 426.72 & $(1400.00)$ & 32.70 \\
\hline 24.38 & $80.00)$ & 55.00 & 609.60 & $(2000.00)$ & 46.70 \\
\hline 30.48 & $100.00)$ & 48.00 & 914.40 & $(3000.00)$ & 52.90 \\
\hline
\end{tabular}

\begin{tabular}{|c|c|c|c|c|}
\hline 0.44 & $1.46)$ & 823.40 & 14.05 & $46.11)$ \\
\hline 0.65 & $2.14)$ & 534.27 & 20.63 & 67.67) \\
\hline 0.96 & $3.14)$ & 242.29 & 30.28 & 99.33) \\
\hline 1.41 & 4.61) & 95.28 & 44.44 & $145.80)$ \\
\hline 2.06 & $6.77)$ & 54.03 & 65.23 & $214.01)$ \\
\hline 3.03 & 9.93) & 41.87 & 95.74 & $314.12)$ \\
\hline 4.44 & $14.58)$ & 35.00 & 140.53 & $461.06)$ \\
\hline 6.52 & $21.40)$ & 40.13 & 206.27 & $676.74)$ \\
\hline \multirow[t]{2}{*}{9.57} & $31.41)$ & 55.44 & 302.77 & $993.32)$ \\
\hline & & & 99999.00 & $(99999.00)$ \\
\hline
\end{tabular}

69.59

74.39

64.66

42.45

22.36

15.13

18.98

32.09

53.41

81.02 

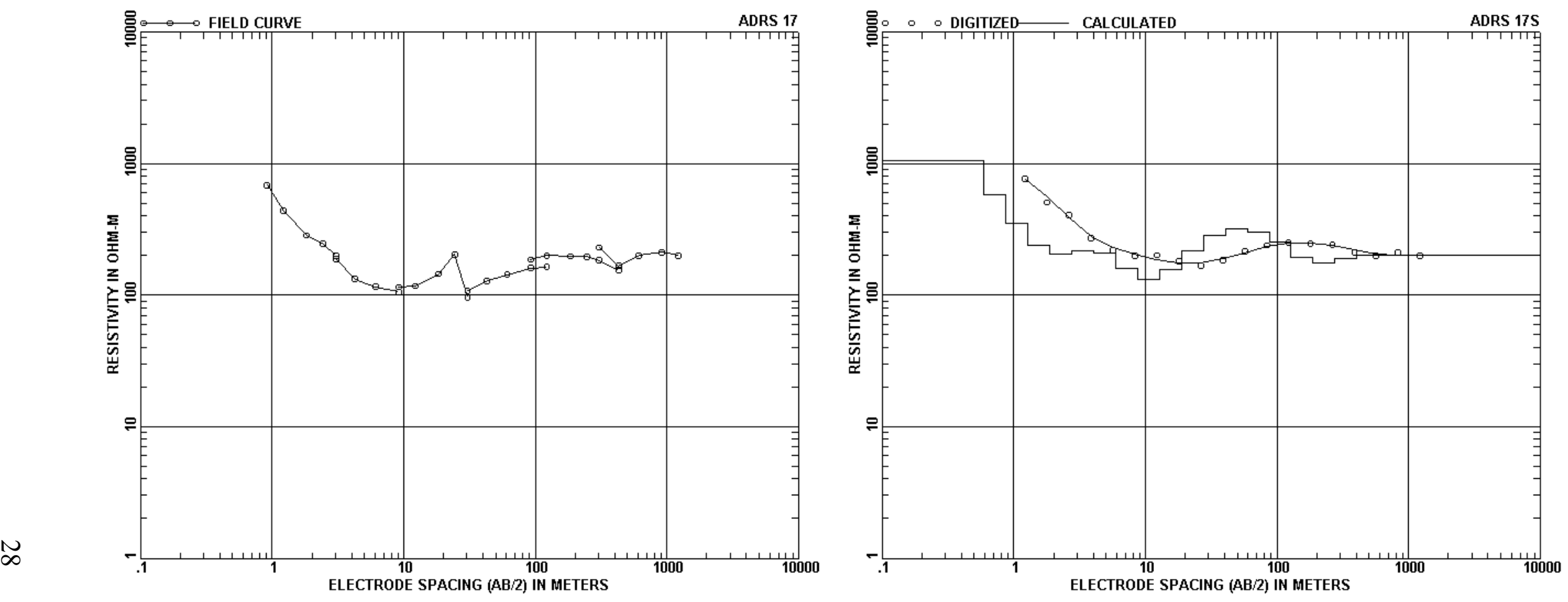

$A B / 2, m$ ( ft ) App. Res. $A B / 2$, m ( ft ) App. Res.

DEPTH, $m$ ( ft ) RESIS. DEPTH, m ( ft ) RESIS.

\begin{tabular}{|c|c|c|c|c|c|}
\hline 0.91 & $3.00)$ & 682.00 & 42.67 & $140.00)$ & 128.00 \\
\hline 1.22 & $4.00)$ & 437.00 & 60.96 & $200.00)$ & 143.00 \\
\hline 1.83 & $6.00)$ & 284.00 & 91.44 & $300.00)$ & 160.00 \\
\hline 2.44 & $8.00)$ & 245.00 & 121.92 & $400.00)$ & 164.0 \\
\hline 3.05 & $10.00)$ & 200.00 & 91.44 & $300.00)$ & 186.00 \\
\hline 3.05 & $10.00)$ & 188.00 & 121.92 & $400.00)$ & 200.00 \\
\hline 4.27 & $14.00)$ & 132.00 & 182.88 & $600.00)$ & 197.0 \\
\hline 6.10 & $20.00)$ & 116.00 & 243.84 & $800.00)$ & 196.0 \\
\hline 9.14 & $30.00)$ & 106.00 & 304.80 & $1000.00)$ & 184.00 \\
\hline 9.14 & $30.00)$ & 115.00 & 426.72 & $1400.00)$ & 154. \\
\hline 12.19 & $40.00)$ & 117.00 & 304.80 & $1000.00)$ & 230.00 \\
\hline 18.29 & $60.00)$ & 145.00 & 426.72 & $1400.00)$ & 167. \\
\hline 24.38 & $80.00)$ & 203.00 & 609.60 & $2000.00)$ & 199. \\
\hline 30.48 & $100.00)$ & 95.80 & 914.40 & $3000.00)$ & 212.0 \\
\hline 30.48 & $100.00)$ & 108.00 & 1219.20 & $4000.00)$ & 200.0 \\
\hline
\end{tabular}

\begin{tabular}{|c|c|c|}
\hline 0.59 & $1.94)$ & 1052.6 \\
\hline 0.87 & 2.85) & 576.8 \\
\hline 1.28 & $4.19)$ & 349.4 \\
\hline 1.87 & $6.15)$ & 237.4 \\
\hline 2.75 & $9.02)$ & 202.8 \\
\hline 4.04 & $13.24)$ & 217.8 \\
\hline 5.93 & $19.44)$ & 207.5 \\
\hline 8.70 & $28.53)$ & 158.5 \\
\hline 12.77 & $41.88)$ & 132. \\
\hline
\end{tabular}

\begin{tabular}{|c|c|c|}
\hline 18.74 & $61.47)$ & 155.58 \\
\hline 27.50 & $90.23)$ & 216.59 \\
\hline 40.37 & $132.44)$ & 281.55 \\
\hline 59.25 & $194.40)$ & 315.72 \\
\hline 86.97 & $285.34)$ & 303.47 \\
\hline 127.66 & $418.82)$ & 250.52 \\
\hline 187.37 & $614.75)$ & 194.40 \\
\hline 275.03 & $902.33)$ & 175.19 \\
\hline 403.69 & $1324.43)$ & 189.49 \\
\hline & $(99999.00)$ & 202.23 \\
\hline
\end{tabular}




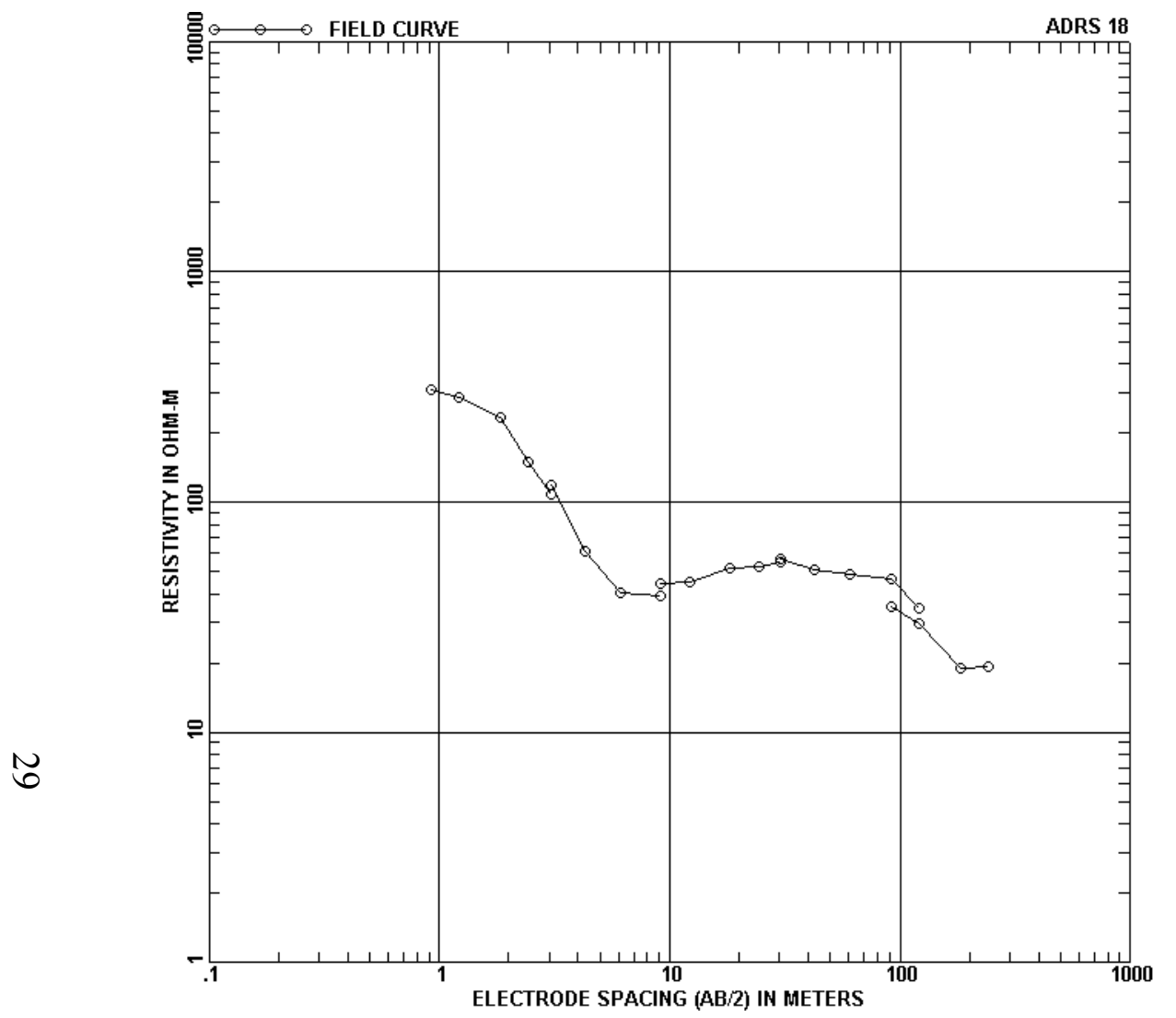

$A B / 2, m(f t)$ App. Res. $A B / 2, m$ ( ft ) App. Res.

\begin{tabular}{|c|c|c|}
\hline 0.91 & $3.00)$ & 308.00 \\
\hline 1.22 & $4.00)$ & 284.00 \\
\hline 1.83 & $6.00)$ & 232.00 \\
\hline 2.44 & $8.00)$ & 149.00 \\
\hline 3.05 & $10.00)$ & 108.00 \\
\hline 3.05 & $10.00)$ & 118.00 \\
\hline 4.27 & $14.00)$ & 61.40 \\
\hline 6.10 & $20.00)$ & 40.30 \\
\hline 9.14 & $30.00)$ & 39.40 \\
\hline 9.14 & $30.00)$ & 44.40 \\
\hline 2.19 & $40.00)$ & 45.20 \\
\hline
\end{tabular}

$18.29(60.00)$ $24.38(80.00)$

$30.48(100.00)$

$30.48(100.00)$

$42.67(140.00)$

$60.96(200.00)$

$91.44(300.00)$

$121.92(400.00)$ $91.44(300.00)$ $182.88(600.00)$ $243.84(800.00)$

51.40

52.40

56.40

51.10

48.20

46.40

34.50

34.50

35.30

29.80

19.00

19.30 $121.92(400.00)$

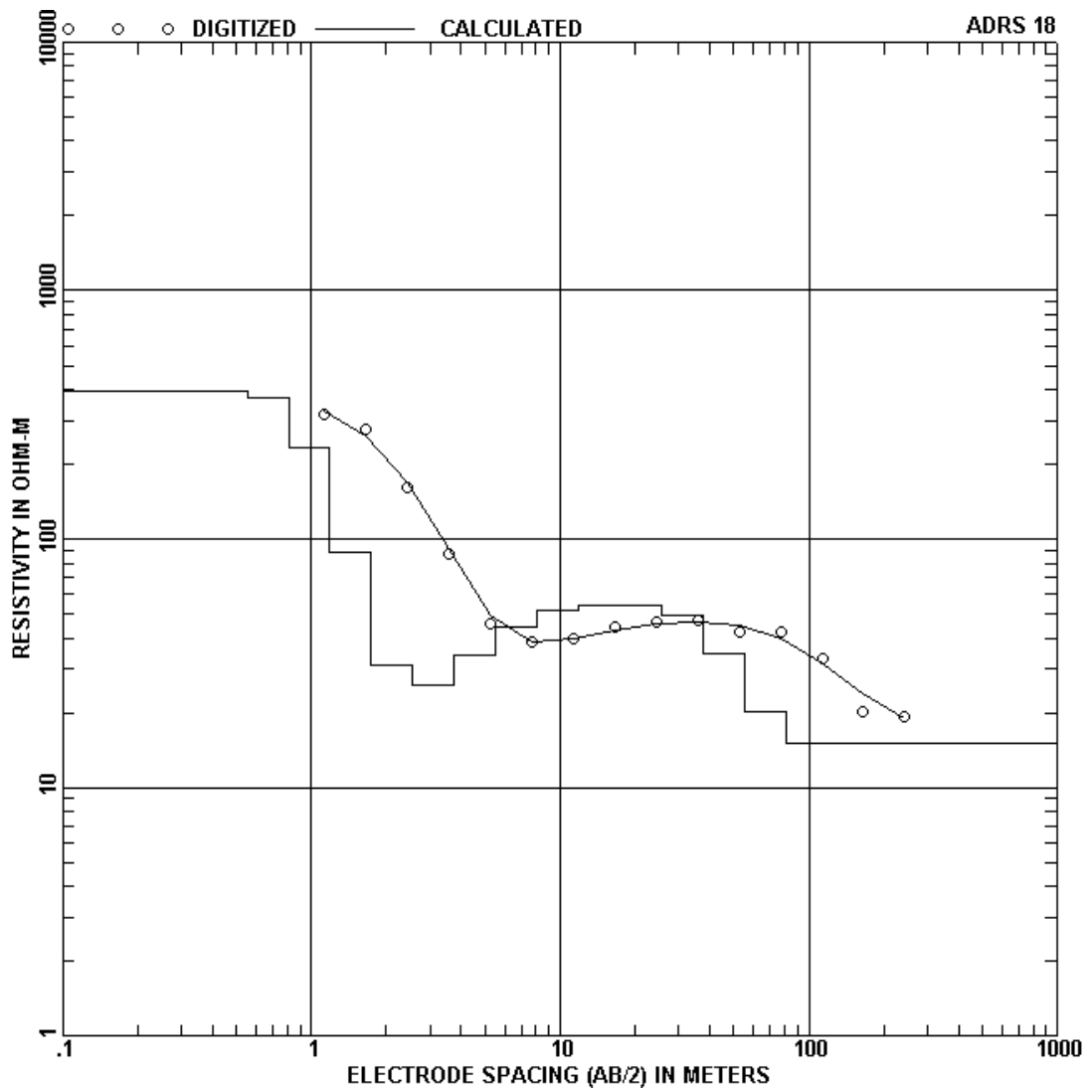

DEPTH, m ( ft ) RESIS. DEPTH, m ( ft ) RESIS.
391.05

369.58

234.21

89.01

31.15

25.72

34.28
44.53

51.31

53.69

54.07

49.57

34.77

20.09 

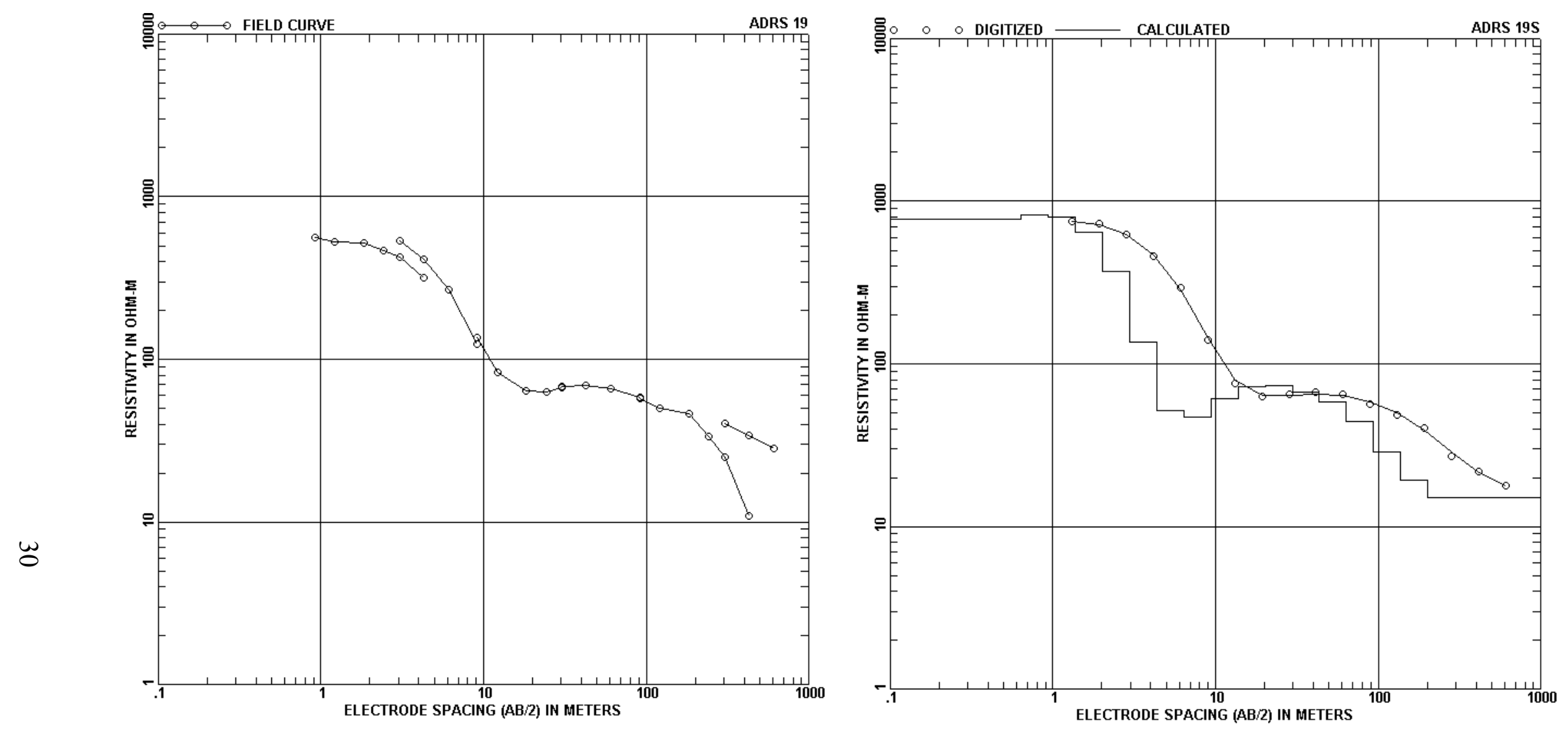

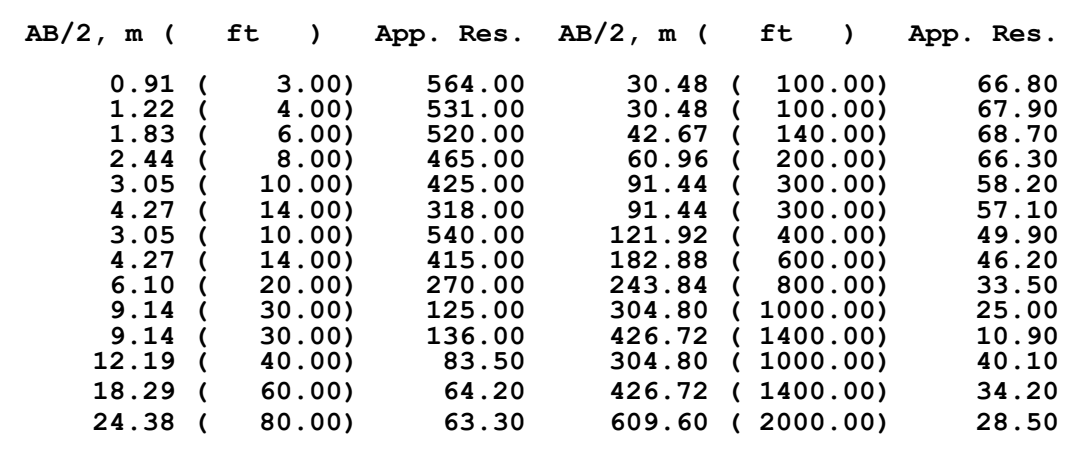

DEPTH, m ( ft )

$\begin{array}{rlr}0.64 & ( & 2.09) \\ 0.94 & ( & 3.07) \\ 1.38 & ( & 4.51) \\ 2.02 & ( & 6.62) \\ 2.96 & ( & 9.72) \\ 4.35 & ( & 14.27) \\ 6.38 & ( & 20.94) \\ 9.37 & ( & 30.74)\end{array}$

RESIS.

772.14

828.66

802.56

643.98

371.31

135.86

51.43

46.96
RESIS.

61.19

72.53

73.71

67.44

58.09

44.13

28.83

19.33

19.33 


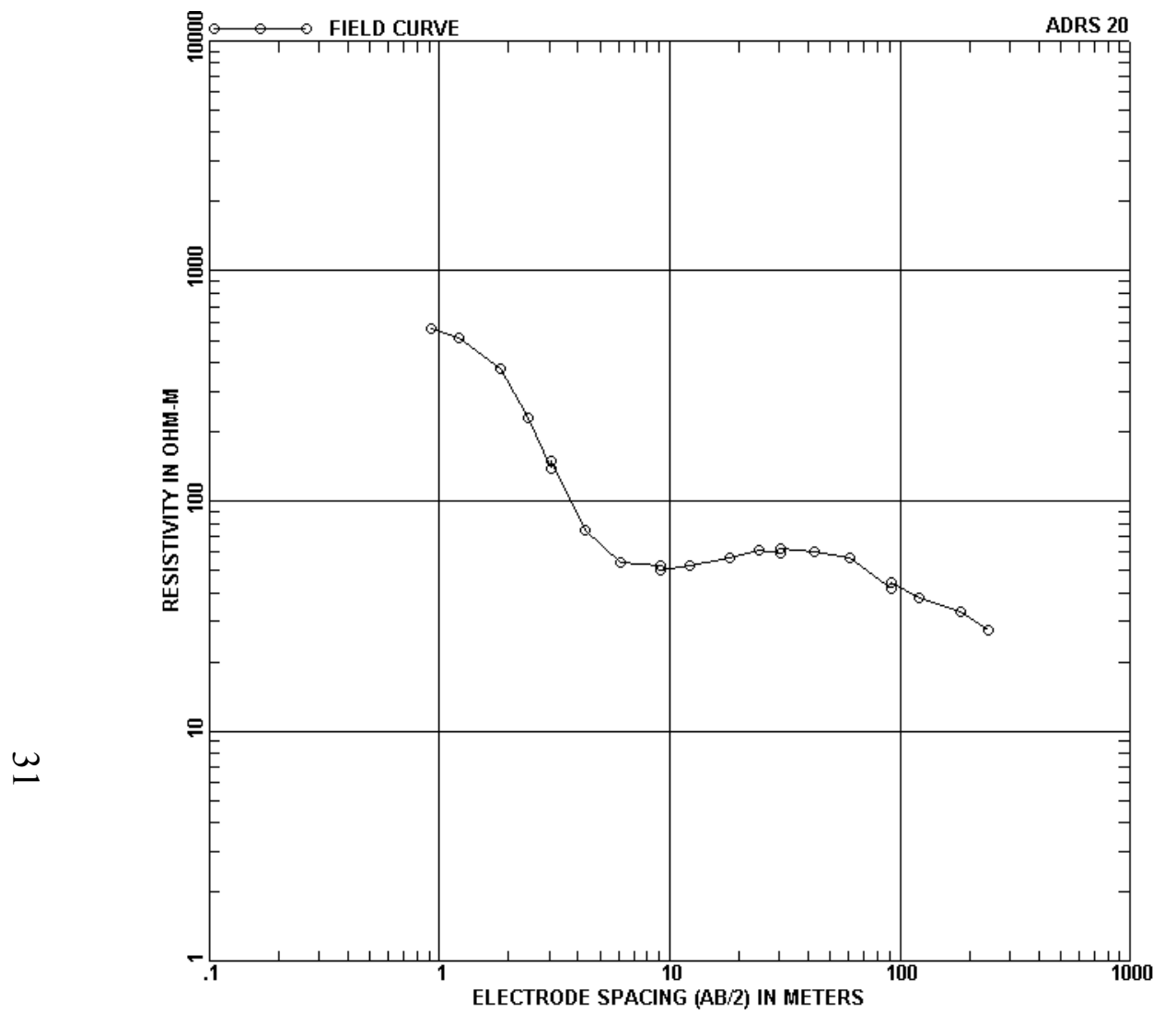

$A B / 2, m$ ( ft ) App. Res. $A B / 2$, m ( ft ) App. Res.

\begin{tabular}{|c|c|c|}
\hline 0.91 & $3.00)$ & 562.00 \\
\hline 1.22 & $4.00)$ & 513.00 \\
\hline 1.83 & $6.00)$ & 377.00 \\
\hline 2.44 & $8.00)$ & 231.00 \\
\hline 3.05 & $10.00)$ & 138.00 \\
\hline 3.05 & $10.00)$ & 150.00 \\
\hline 4.27 & $14.00)$ & 75.20 \\
\hline 6.10 & $20.00)$ & 53.90 \\
\hline 9.14 & $30.00)$ & 52.20 \\
\hline 9.14 & $30.00)$ & 50.30 \\
\hline 2.19 & $40.00)$ & 52.60 \\
\hline
\end{tabular}

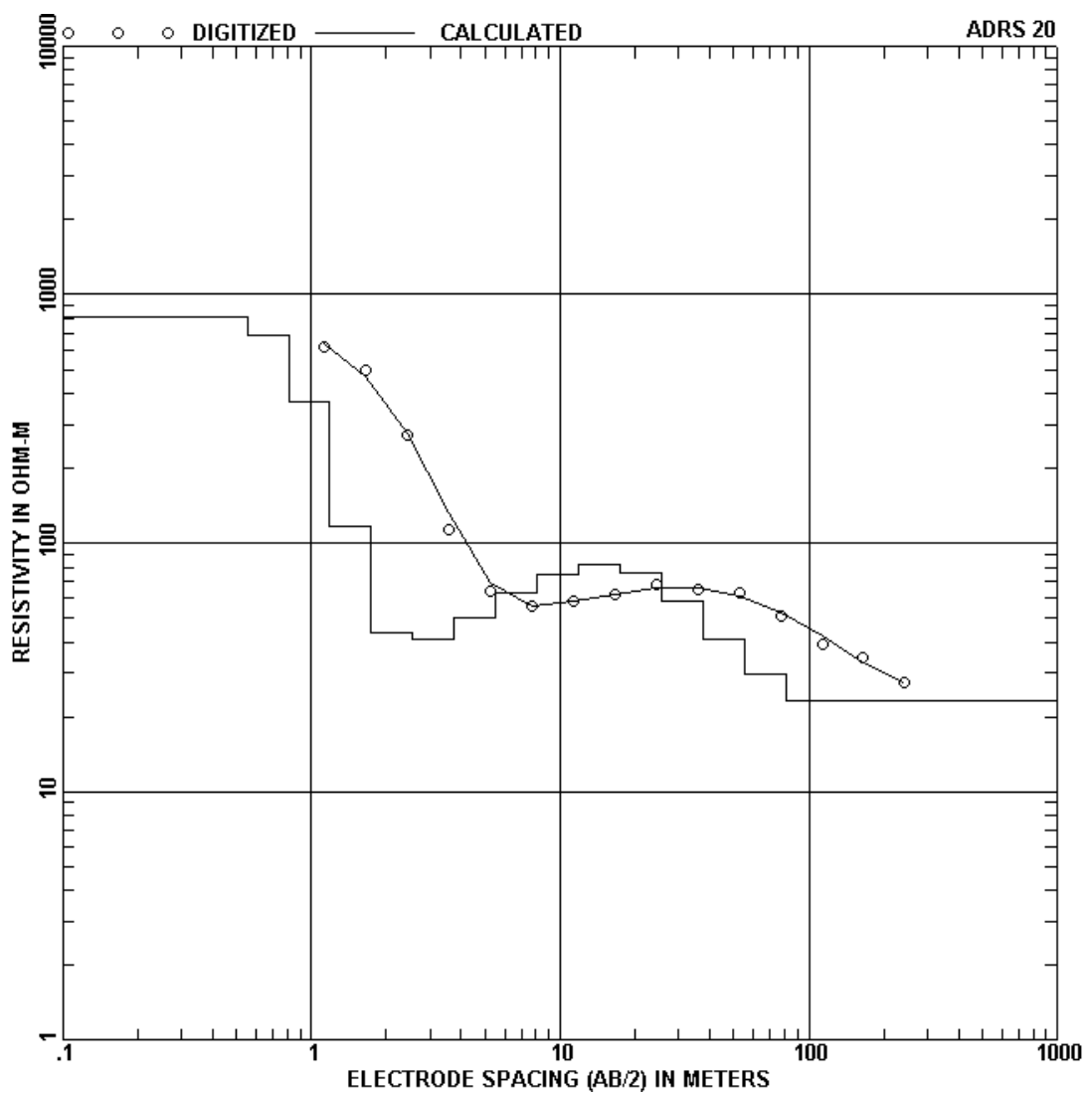

DEPTH, $m$ ( ft ) RESIS. DEPTH, $m($ ft ) RESIS.

\begin{tabular}{|c|c|}
\hline .55 & $1.80)$ \\
\hline 0.81 & $2.65)$ \\
\hline 1.1 & $3.89)$ \\
\hline L. & 5. \\
\hline 2.5 & $8.38)$ \\
\hline .7 & $12.29)$ \\
\hline 5 & $18.05)$ \\
\hline
\end{tabular}

809.31

687.48

370.81

116.71

43.30

40.78

50.25
62.60

74.97

81.39

75.32

58.16

40.89

29.69

23.18 


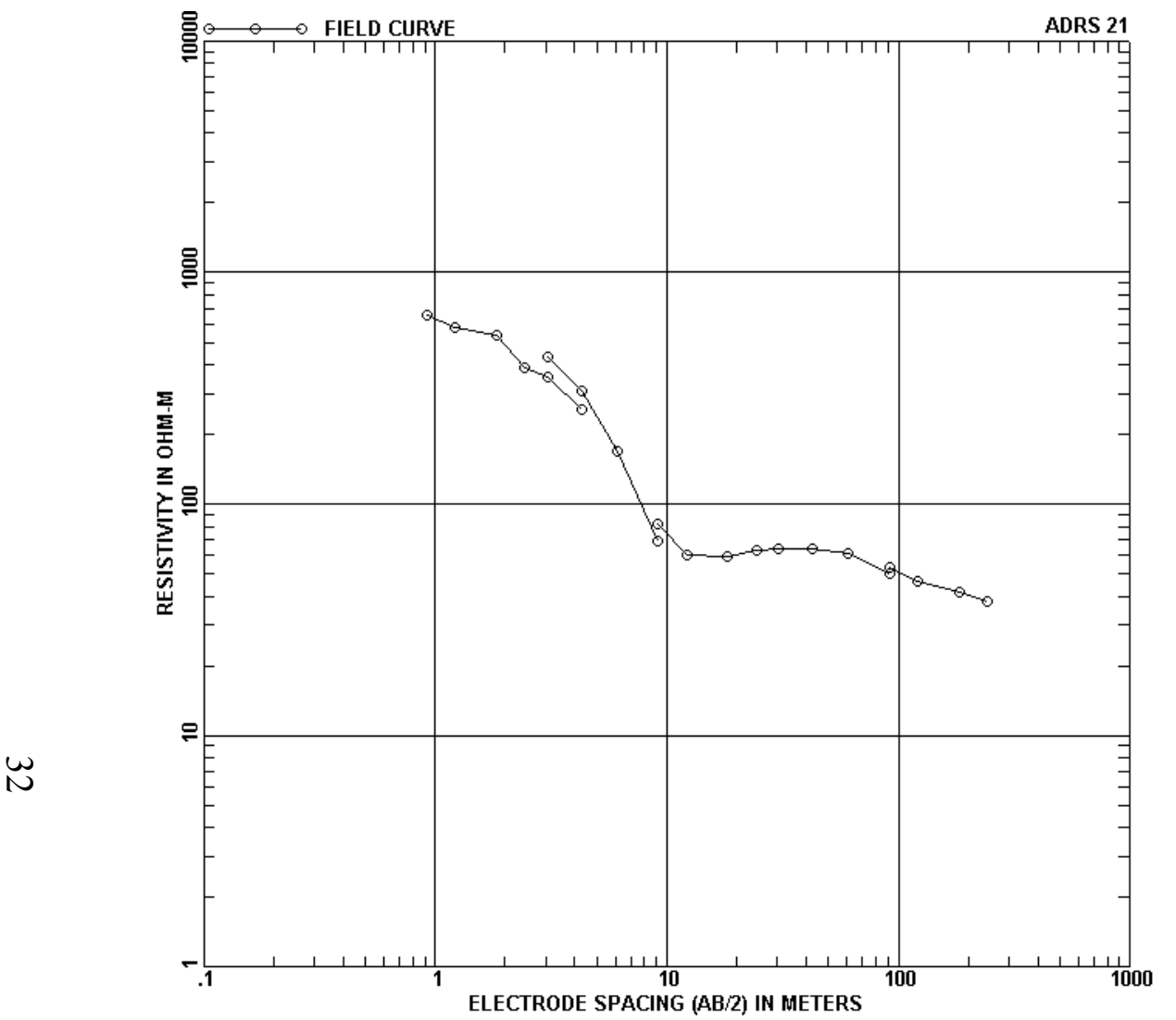

$A B / 2, m($ ft ) App. Res. $A B / 2, m($ ft ) App. Res.

\begin{tabular}{|c|c|c|}
\hline 0.91 & $3.00)$ & 659.00 \\
\hline 1.22 & $4.00)$ & 579.00 \\
\hline 1.83 & $6.00)$ & 532.00 \\
\hline 2.44 & $8.00)$ & 388.00 \\
\hline 3.05 & $10.00)$ & 352.00 \\
\hline 4.27 & $14.00)$ & 256.00 \\
\hline 3.05 & $10.00)$ & 431.00 \\
\hline 4.27 & $14.00)$ & 309.00 \\
\hline 6.10 & $20.00)$ & 170.00 \\
\hline 9.14 & $30.00)$ & 69.30 \\
\hline 9.14 & $30.00)$ & 81.30 \\
\hline
\end{tabular}

\begin{tabular}{|c|c|c|}
\hline 12.19 & $40.00)$ & 60.10 \\
\hline 18.29 & $60.00)$ & 59.60 \\
\hline 24.38 & $80.00)$ & 63.30 \\
\hline 30.48 & $100.00)$ & 64.10 \\
\hline 30.48 & $100.00)$ & 64.00 \\
\hline 42.67 & $140.00)$ & 63.70 \\
\hline 60.96 & $200.00)$ & 60.70 \\
\hline 91.44 & $300.00)$ & 50.1 \\
\hline 91.44 & $300.00)$ & 53.40 \\
\hline 121.92 & $400.00)$ & $46.6 c$ \\
\hline 182.88 & $600.00)$ & 41.6 \\
\hline 243.84 & $800.00)$ & 38.1 \\
\hline
\end{tabular}

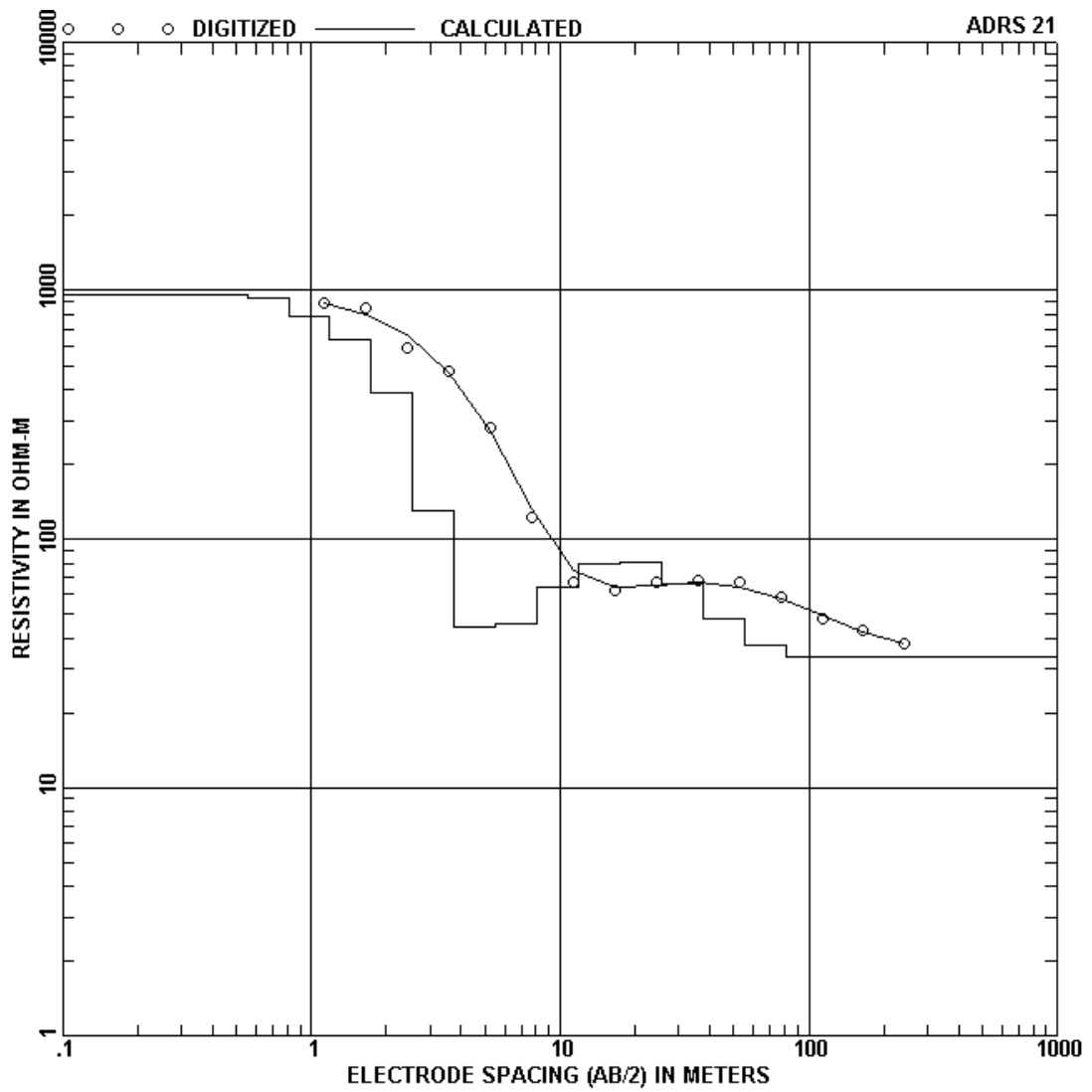

DEPTH, $m($ ft ) RESIS. DEPTH, m ( ft ) RESIS

\begin{tabular}{|c|c|c|}
\hline 0.55 & $1.80)$ & 964.98 \\
\hline 0.81 & $2.65)$ & 929.65 \\
\hline 1.19 & $3.89)$ & 790.15 \\
\hline 1.74 & $5.71)$ & 633.56 \\
\hline 2.55 & $8.38)$ & 389.16 \\
\hline 3.75 & $12.29)$ & 130.76 \\
\hline 5.50 & $18.05)$ & 44.40 \\
\hline
\end{tabular}

$8.07(-26.49)$ $11.85(38.88)$ $17.39(57.07)$ $25.53(83.76)$ $37.47(122.95)$ $55.01(180.47)$ $80.74(264.89)$ $99999.00 \quad(99999.00)$
45.61 64.46 79.50 80.28 65.64 47.84 37.22 33.66 


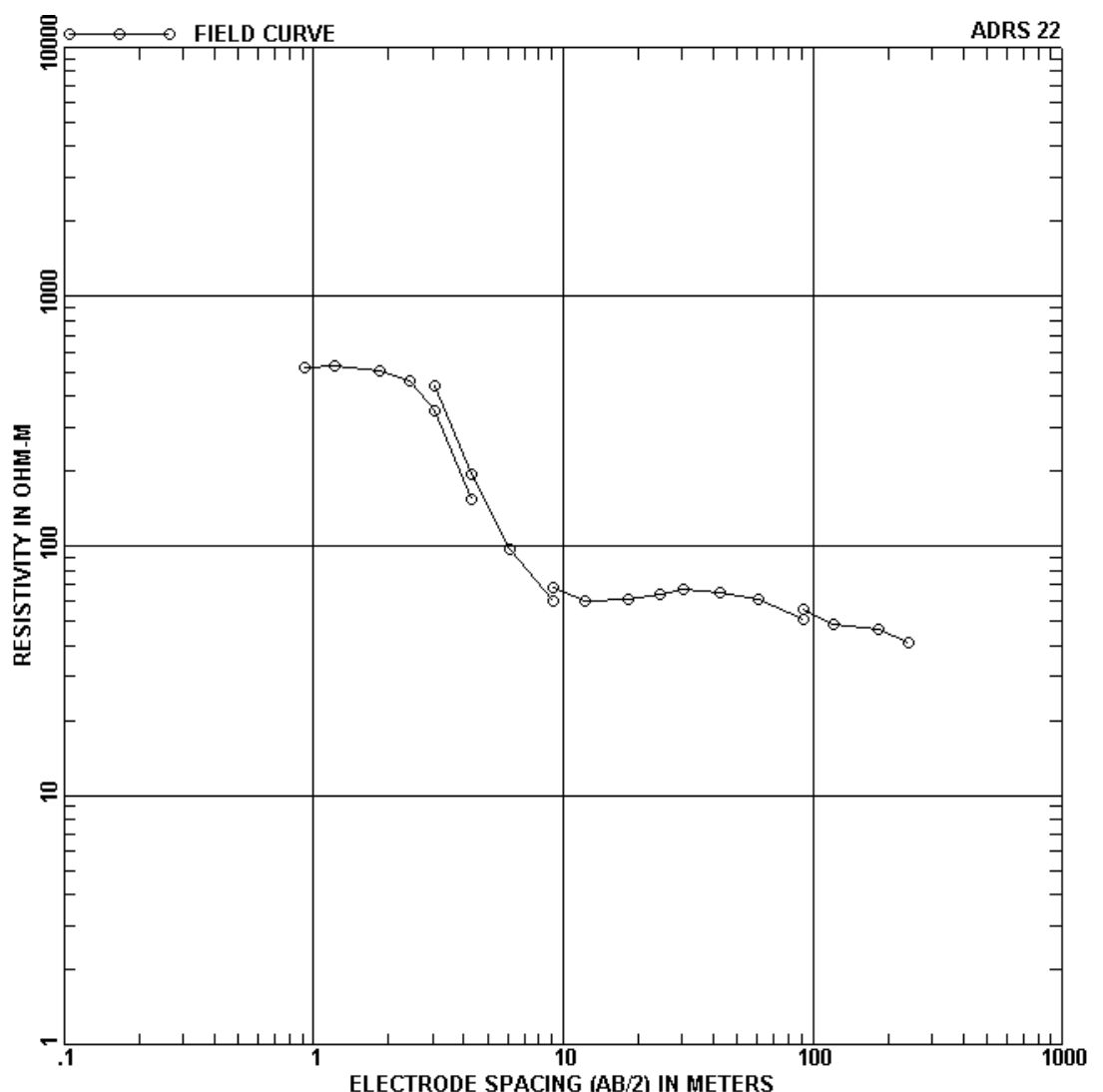

$A B / 2$, m ( ft ) App. Res. $A B / 2$, m ( ft ) App. Res.

\begin{tabular}{|c|c|c|}
\hline 0.91 & $3.00)$ & 521.00 \\
\hline 1.22 & $4.00)$ & 531.00 \\
\hline 1.83 & $6.00)$ & 505.00 \\
\hline 2.44 & $8.00)$ & 458.00 \\
\hline 3.05 & $10.00)$ & 347.00 \\
\hline 4.27 & $14.00)$ & 153.00 \\
\hline 3.05 & $10.00)$ & 438.00 \\
\hline 4.27 & $14.00)$ & 194.00 \\
\hline 6.10 & $20.00)$ & 96.40 \\
\hline 9.14 & $30.00)$ & 60.10 \\
\hline 9.14 & $30.00)$ & 68.10 \\
\hline
\end{tabular}

$91.44(300.00)$

1.10 121.92( $182.88(600.00)$

8.20

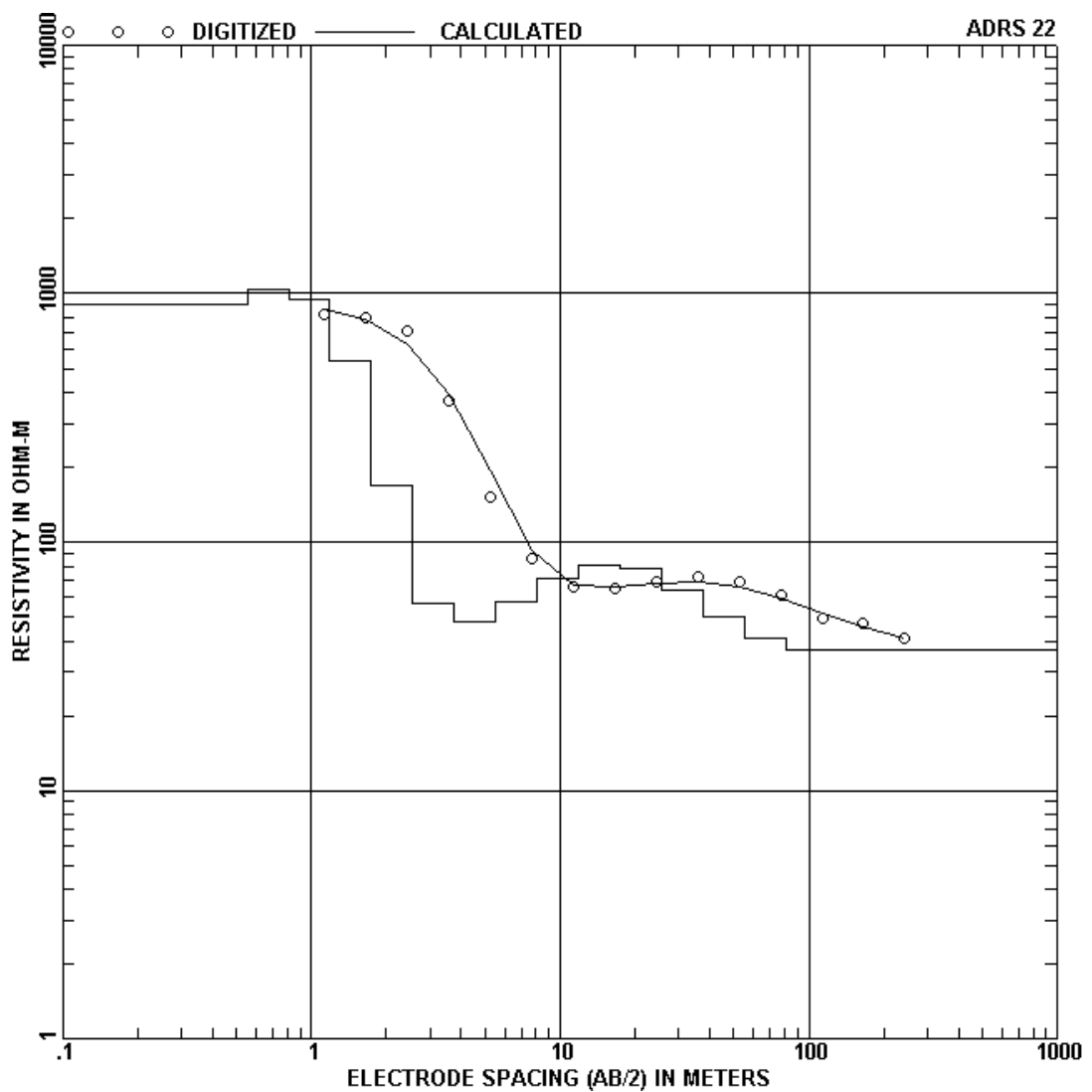

DEPTH, m ( ft ) RESIS. DEPTH, m ( ft ) RESIS.

60.00
61.10
64.30
67.20
66.90
65.20
61.50
51.10
55.40
48.20
46.50
41.00

\begin{tabular}{|c|c|}
\hline 55 & $1.80)$ \\
\hline .0 & $65)$ \\
\hline .1 & $3.89)$ \\
\hline 7 & $5.71)$ \\
\hline .5 & $8.38)$ \\
\hline 7 & $12.29)$ \\
\hline & $18.05)$ \\
\hline
\end{tabular}

900.89

1040.08

952.64

532.79

168.98

56.98

47.70
57.82

71.49

80.84

77.86

63.95

49.76

41.26

37.06
41.00 


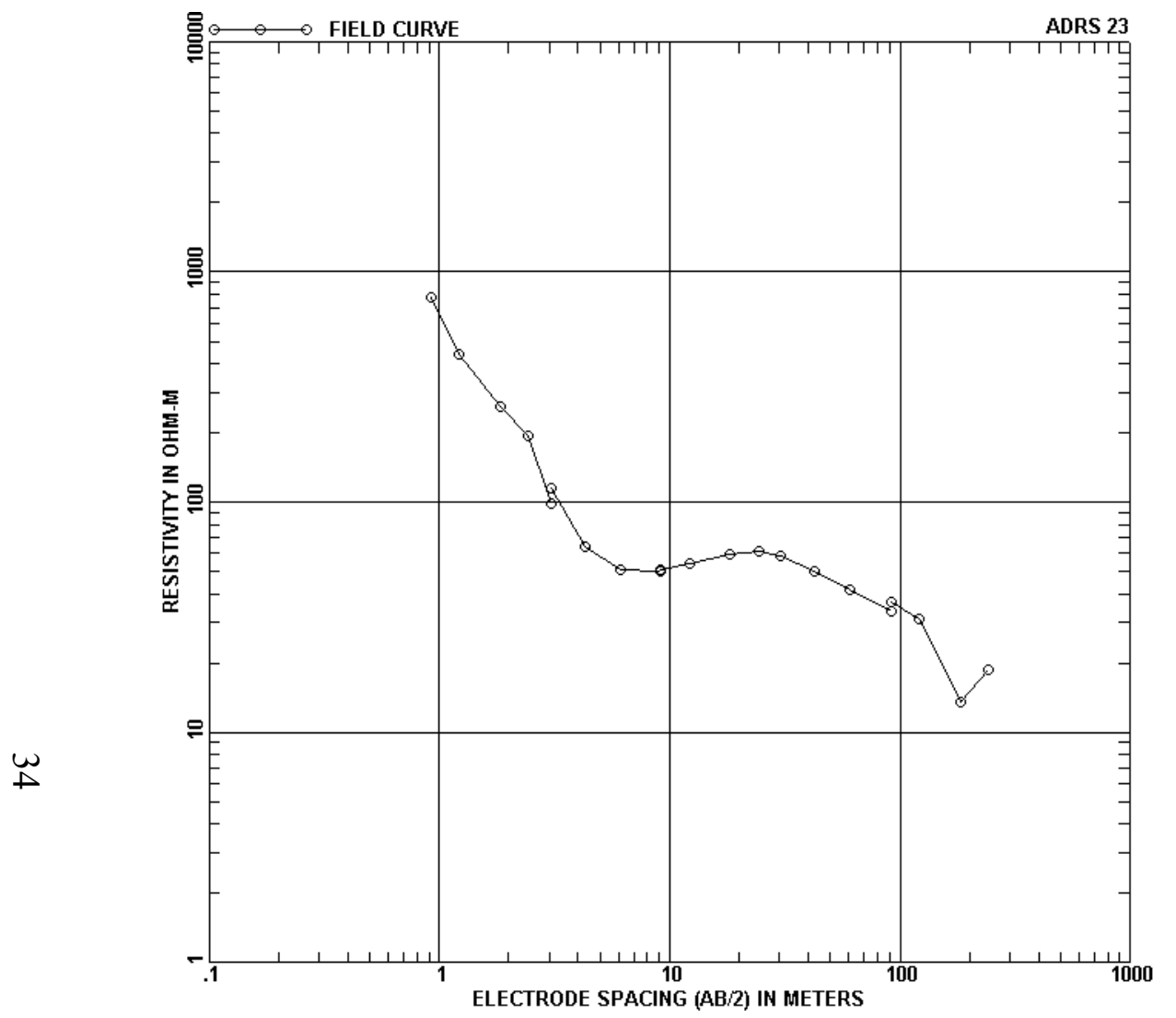

$A B / 2, m(f t)$ App. Res. $A B / 2, m$ ( ft ) App. Res.

\begin{tabular}{|c|c|c|}
\hline 0.91 & $3.00)$ & 773.00 \\
\hline 1.22 & $4.00)$ & 442.00 \\
\hline 1.83 & $6.00)$ & 259.00 \\
\hline 2.44 & $8.00)$ & 194.00 \\
\hline 3.05 & $10.00)$ & 98.60 \\
\hline 3.05 & $10.00)$ & 115.00 \\
\hline 4.27 & $14.00)$ & 63.70 \\
\hline 6.10 & $20.00)$ & 50.70 \\
\hline 9.14 & $30.00)$ & 50.10 \\
\hline 9.14 & $30.00)$ & 50.50 \\
\hline 2.19 & $40.00)$ & 54.20 \\
\hline
\end{tabular}

\begin{tabular}{|c|c|}
\hline 29 & 0) \\
\hline 24. & \\
\hline .2 & 100 \\
\hline & 10 \\
\hline . & 140 \\
\hline.$\varsigma$ & 200 \\
\hline 91.44 & 300 \\
\hline 91.44 & 300 \\
\hline 1 & 0) \\
\hline 28 & 600 \\
\hline 43.8 & 800.00 \\
\hline
\end{tabular}

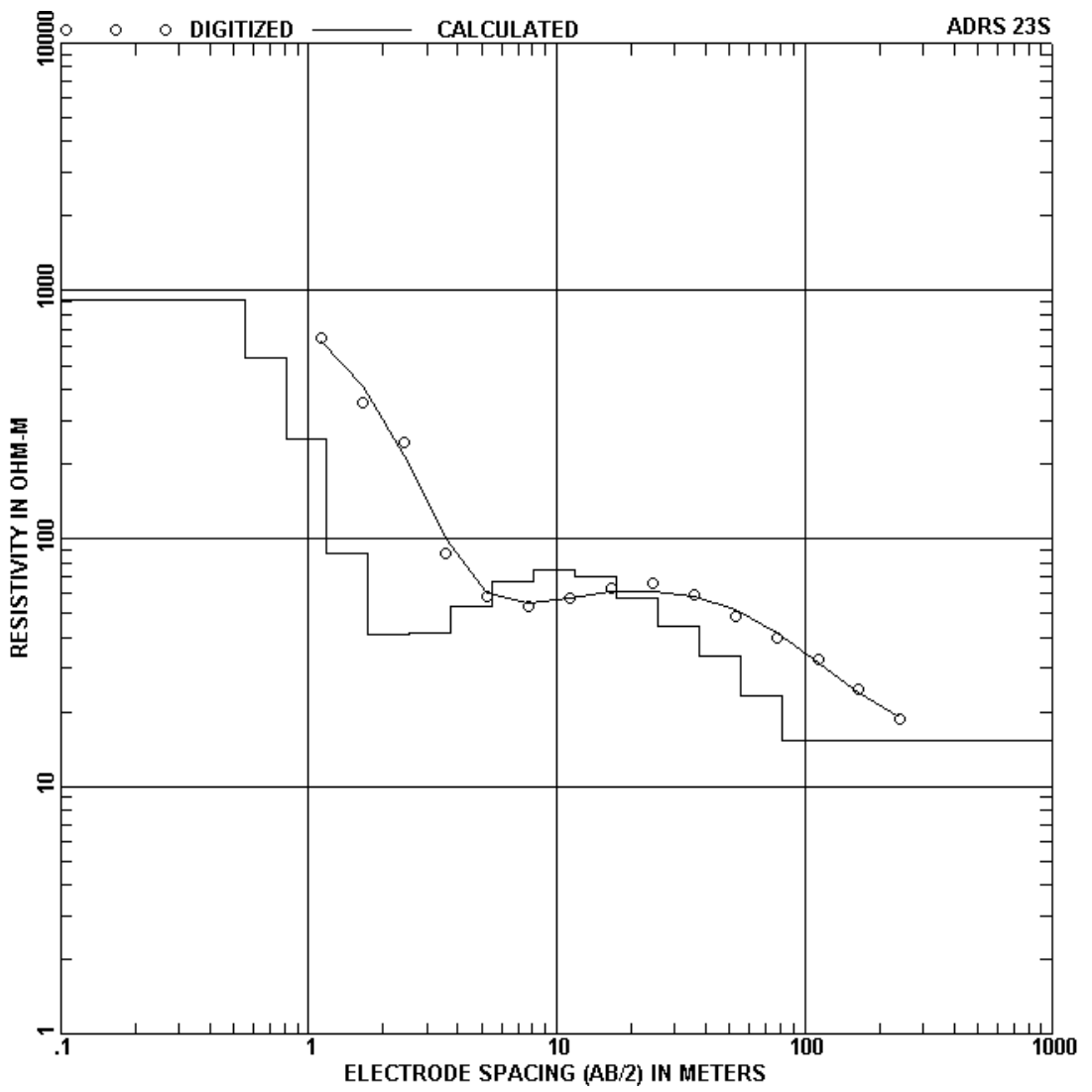

DEPTH, $m$ ( ft ) RESIS. DEPTH, m ( ft ) RESIS.

\begin{tabular}{|c|c|}
\hline 0.55 & 1 \\
\hline 0.81 & . \\
\hline 1.19 & \\
\hline 1.74 & \\
\hline 2.55 & 8. \\
\hline 3.75 & 12.2 \\
\hline 5.50 & 18. \\
\hline
\end{tabular}

)

\begin{tabular}{|c|c|}
\hline 8.07 & $26.49)$ \\
\hline 11.85 & $38.88)$ \\
\hline 17.39 & $57.07)$ \\
\hline 25.53 & $83.76)$ \\
\hline 37.47 & $122.95)$ \\
\hline 55.01 & $180.47)$ \\
\hline 80.74 & $264.89)$ \\
\hline 999.00 & $(99999.00)$ \\
\hline
\end{tabular}

66.64

74.22

70.08

57.51

44.37

33.42

23.22

15.33 


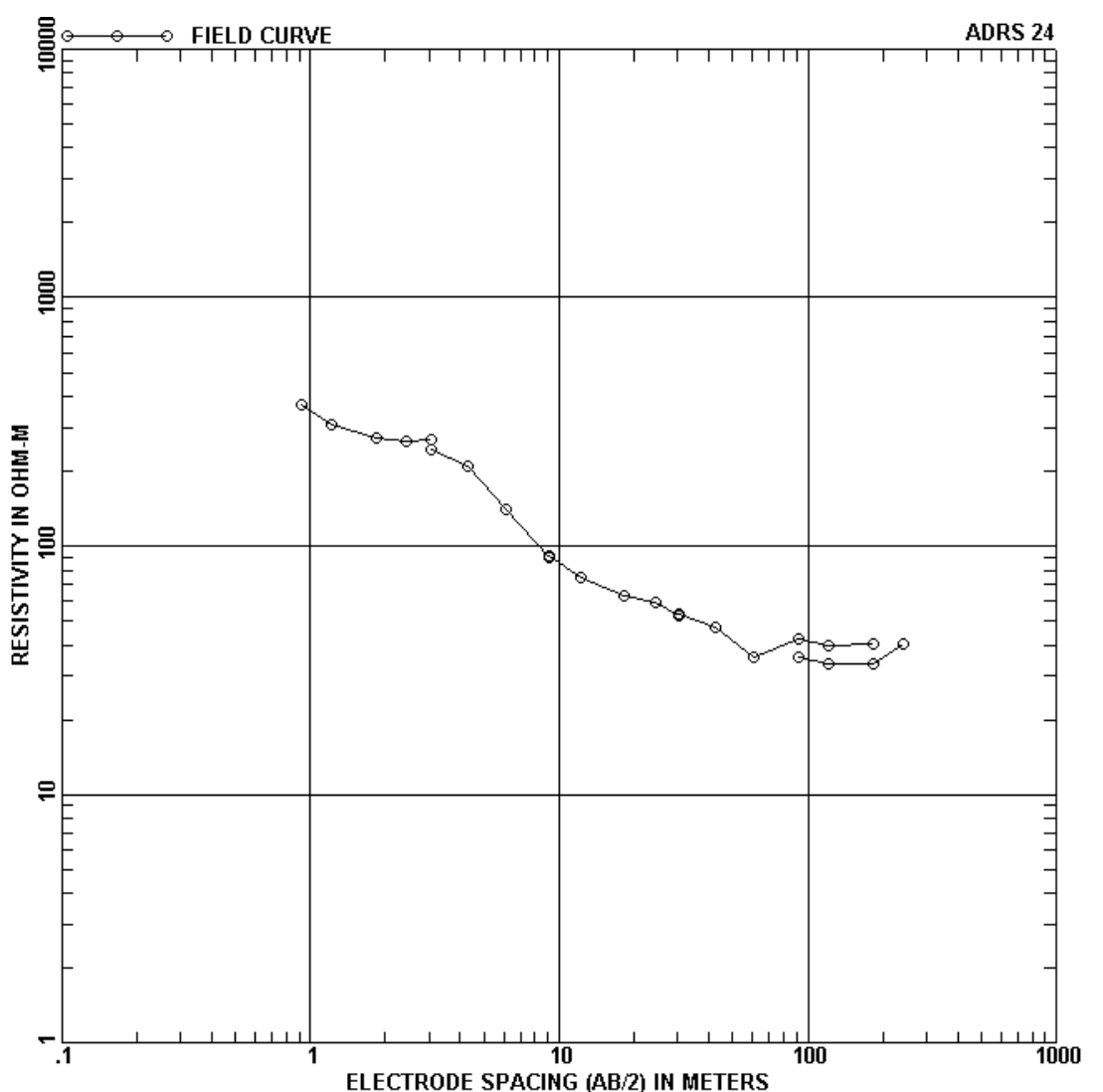

$A B / 2$, $m$ ( ft ) App. Res. $A B / 2, m$ ( ft ) App. Res.

\begin{tabular}{|c|c|c|}
\hline 0.91 & $3.00)$ & 371.00 \\
\hline 1.22 & $4.00)$ & 307.00 \\
\hline 1.83 & $6.00)$ & 273.00 \\
\hline 2.44 & $8.00)$ & 264.00 \\
\hline 3.05 & $10.00)$ & 267.00 \\
\hline 3.05 & $10.00)$ & 245.00 \\
\hline 4.27 & $14.00)$ & 210.00 \\
\hline 6.10 & $20.00)$ & 141.00 \\
\hline 9.14 & $30.00)$ & 90.30 \\
\hline 9.14 & $30.00)$ & 91.30 \\
\hline 12.19 & $40.00)$ & 74.60 \\
\hline 18.29 & $60.00)$ & 63.30 \\
\hline
\end{tabular}

59.20
52.50
53.60
47.10
35.70
42.00
39.50
40.60
35.80
33.50
33.30
40.40
$121.92(400.00)$

182.88 ( 600.00$)$

33.30

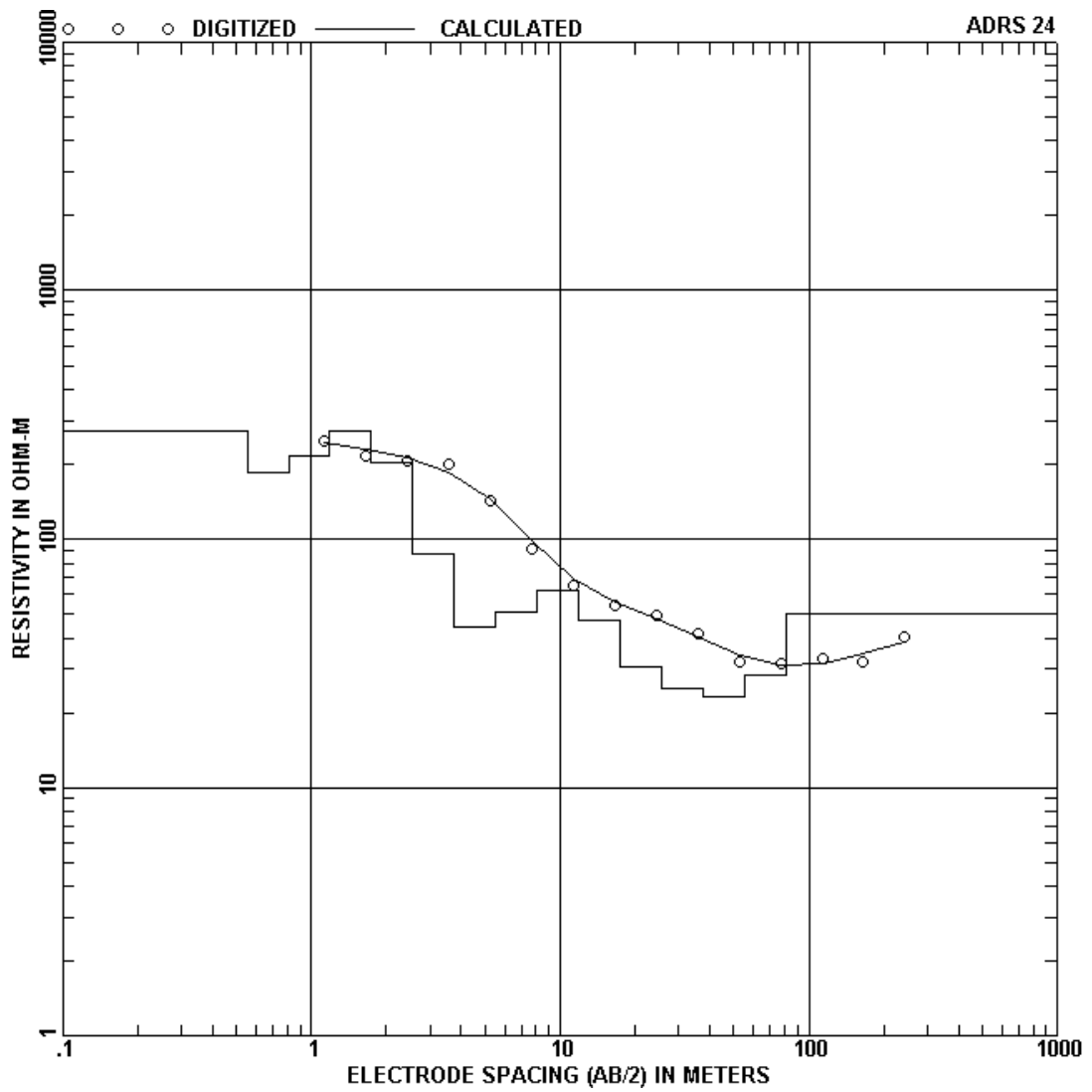

DEPTH, m ( ft ) RESIS. DEPTH, m ( ft ) RESIS.

\begin{tabular}{|c|c|}
\hline 55 & $1.80)$ \\
\hline .0 & $65)$ \\
\hline .1 & $3.89)$ \\
\hline 7 & $5.71)$ \\
\hline .5 & $8.38)$ \\
\hline 7 & $12.29)$ \\
\hline & $18.05)$ \\
\hline
\end{tabular}

273.74

186.41

215.12

271.03

204.50

44.20

$8.07(\quad 26.49)$
$11.85(\quad 38.88)$

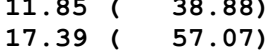

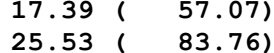

80.74 ( 264.89)

50.95

62.22

47.28

30.61

25.02

23.21

28.17

49.95 $99999.00 \quad(99999.00)$
$37.47(122.95)$

55.01 ( 180.47) 

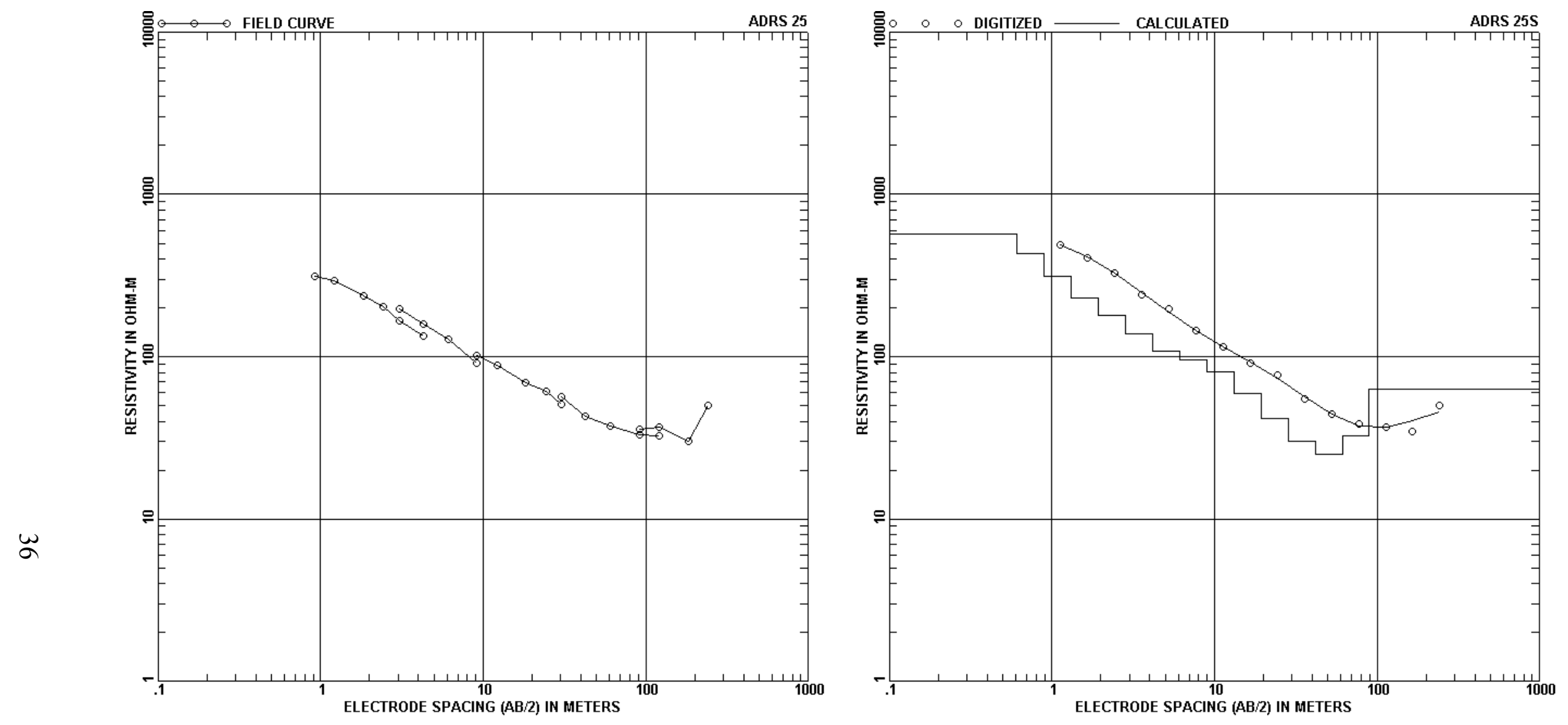

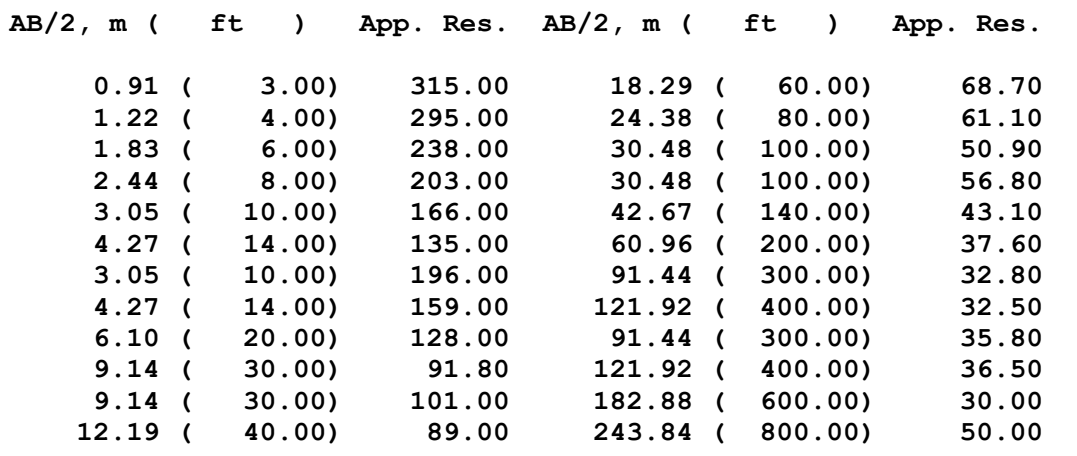

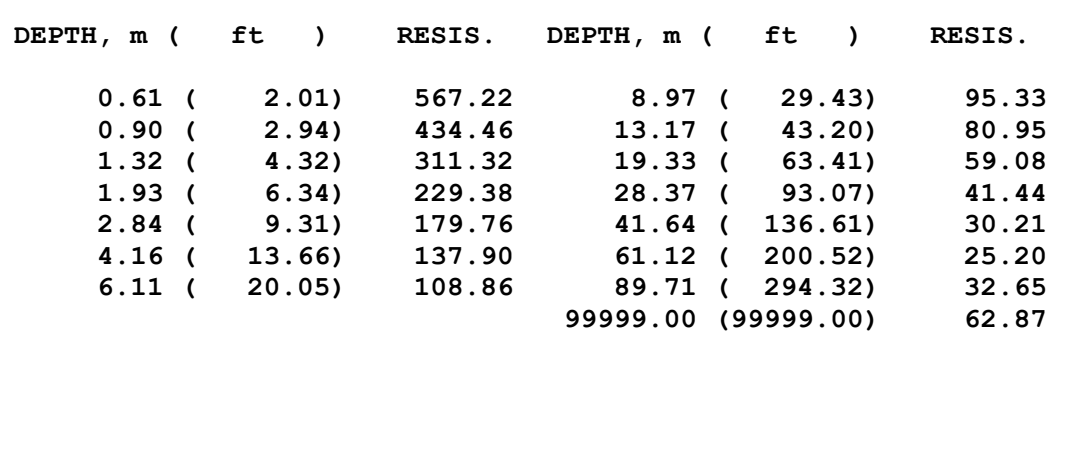




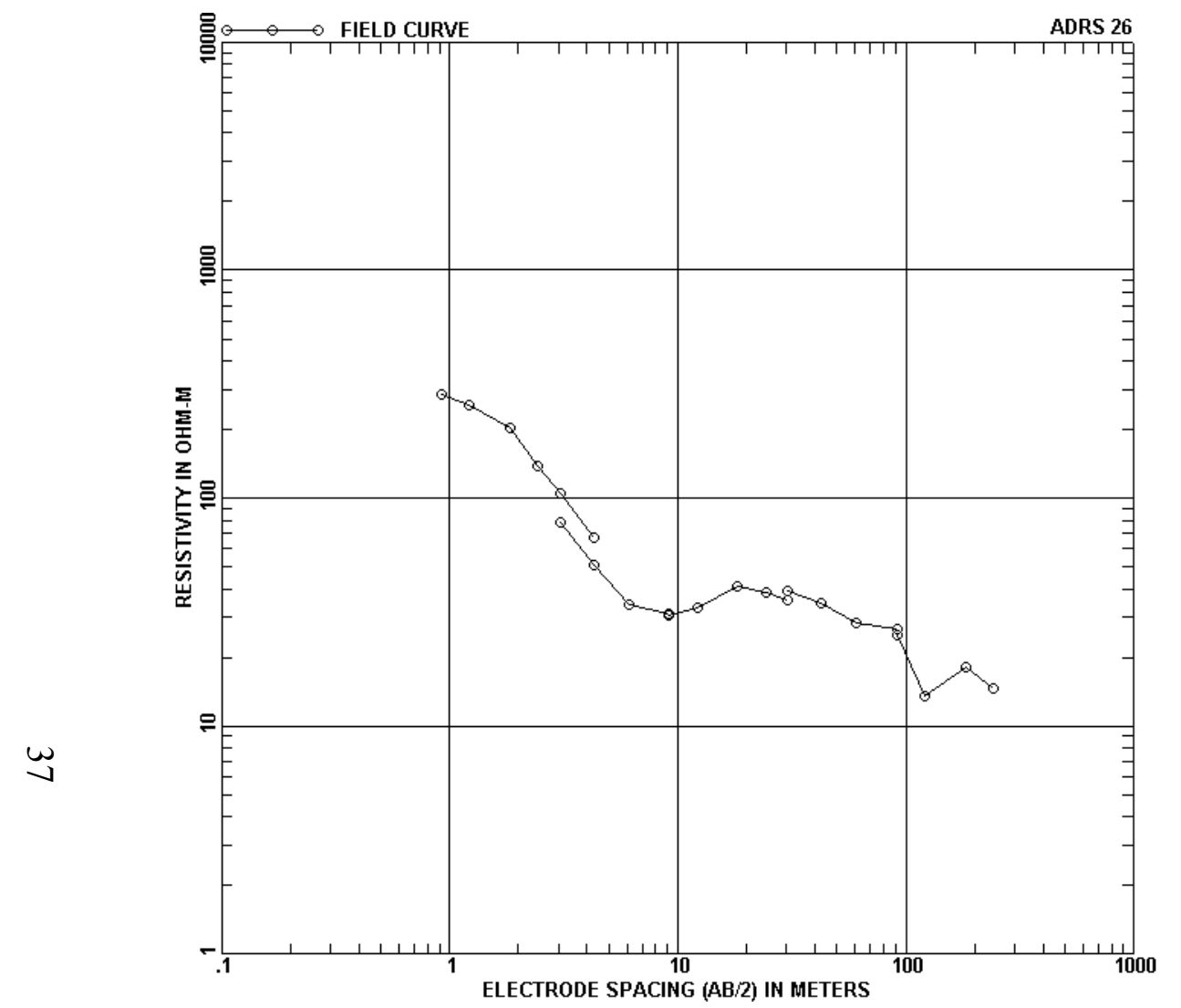

$A B / 2, m$ ( ft ) App. Res. $A B / 2, m$ ( ft ) App. Res.

\begin{tabular}{|c|c|c|}
\hline 0.91 & $3.00)$ & 285.00 \\
\hline 1.22 & $4.00)$ & 256.00 \\
\hline 1.83 & $6.00)$ & 204.00 \\
\hline 2.44 & $8.00)$ & 139.00 \\
\hline 3.05 & $10.00)$ & 104.00 \\
\hline 4.27 & $14.00)$ & 67.50 \\
\hline 3.05 & $10.00)$ & 77.70 \\
\hline 4.27 & $14.00)$ & 50.50 \\
\hline 6.10 & $20.00)$ & 34.20 \\
\hline 9.14 & $30.00)$ & 31.20 \\
\hline 9.14 & $30.00)$ & 30.70 \\
\hline
\end{tabular}

\begin{tabular}{|c|c|c|}
\hline 12.19 & $40.00)$ & 33.10 \\
\hline 18.29 & $60.00)$ & 40.80 \\
\hline 24.38 & $80.00)$ & 38.30 \\
\hline 30.48 & $100.00)$ & 35.40 \\
\hline 30.48 & $100.00)$ & 39.40 \\
\hline 42.67 & $140.00)$ & 34.50 \\
\hline 60.96 & $200.00)$ & 28.4 \\
\hline 91.44 & $300.00)$ & 26.8 \\
\hline 91.44 & $300.00)$ & 24.9 \\
\hline 121.92 & $400.00)$ & 13.6 \\
\hline 182.88 & $600.00)$ & 18.1 \\
\hline 243.84 & $800.00)$ & 14.5 \\
\hline
\end{tabular}

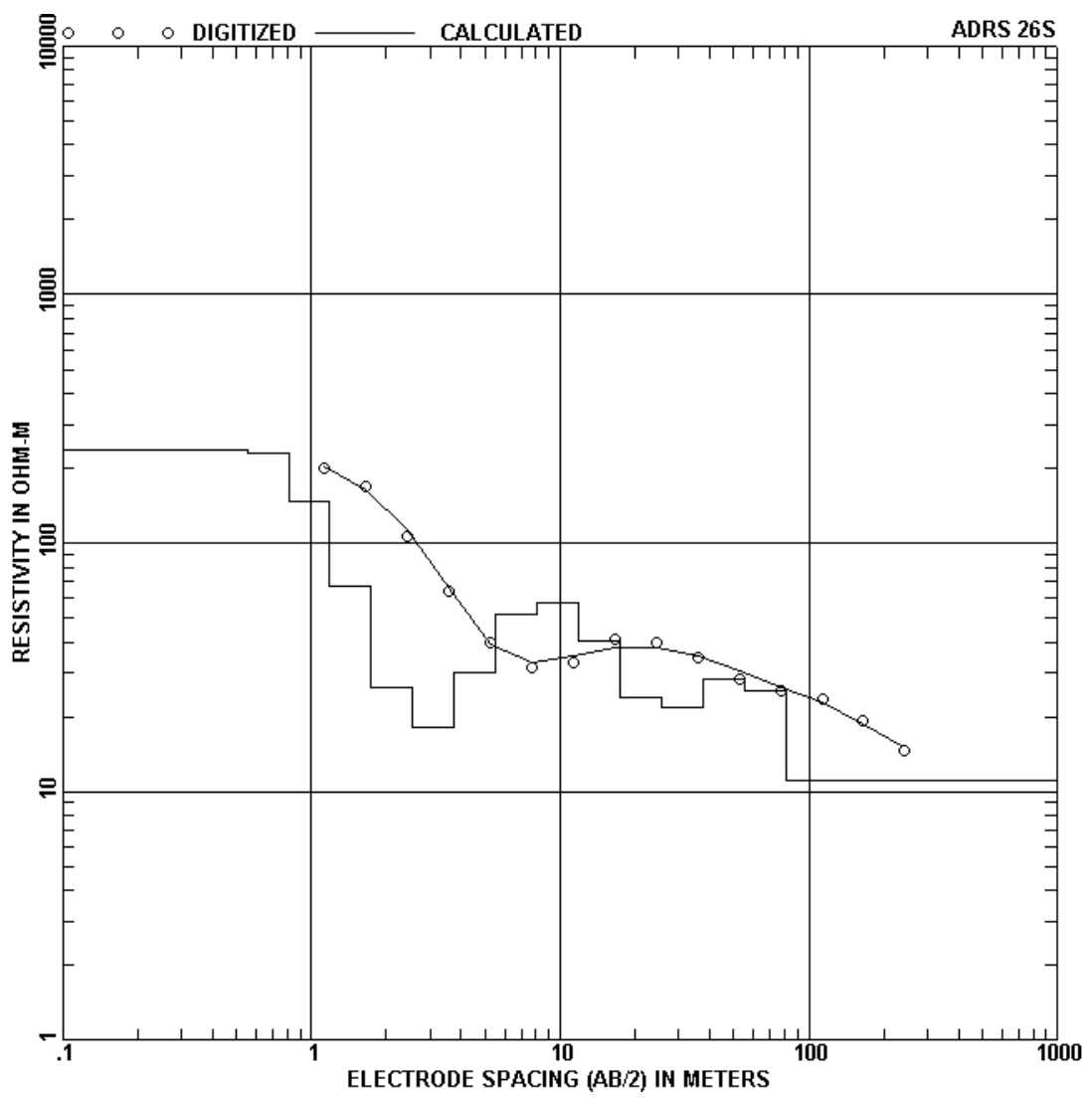

DEPTH, m ( ft ) RESIS. DEPTH, $m$ ( ft ) RESIS.

$\begin{array}{rlr}0.55 & ( & 1.80) \\ 0.81 & ( & 2.65) \\ 1.19 & ( & 3.89) \\ 1.74 & ( & 5.71) \\ 2.55 & ( & 8.38) \\ 3.75 & ( & 12.29) \\ 5.50 & ( & 18.05)\end{array}$

238.33

231.35

148.14

66.57

26.28

18.17

\begin{tabular}{|c|c|}
\hline 8.07 & $26.49)$ \\
\hline 11.85 & $38.88)$ \\
\hline 17.39 & $57.07)$ \\
\hline 25.53 & $83.76)$ \\
\hline 37.47 & $122.95)$ \\
\hline 55.01 & $180.47)$ \\
\hline 80.74 & $264.89)$ \\
\hline 99.00 & $999.00)$ \\
\hline
\end{tabular}

51.67

57.53

40.05

23.93

21.67

28.29

25.29

10.99 


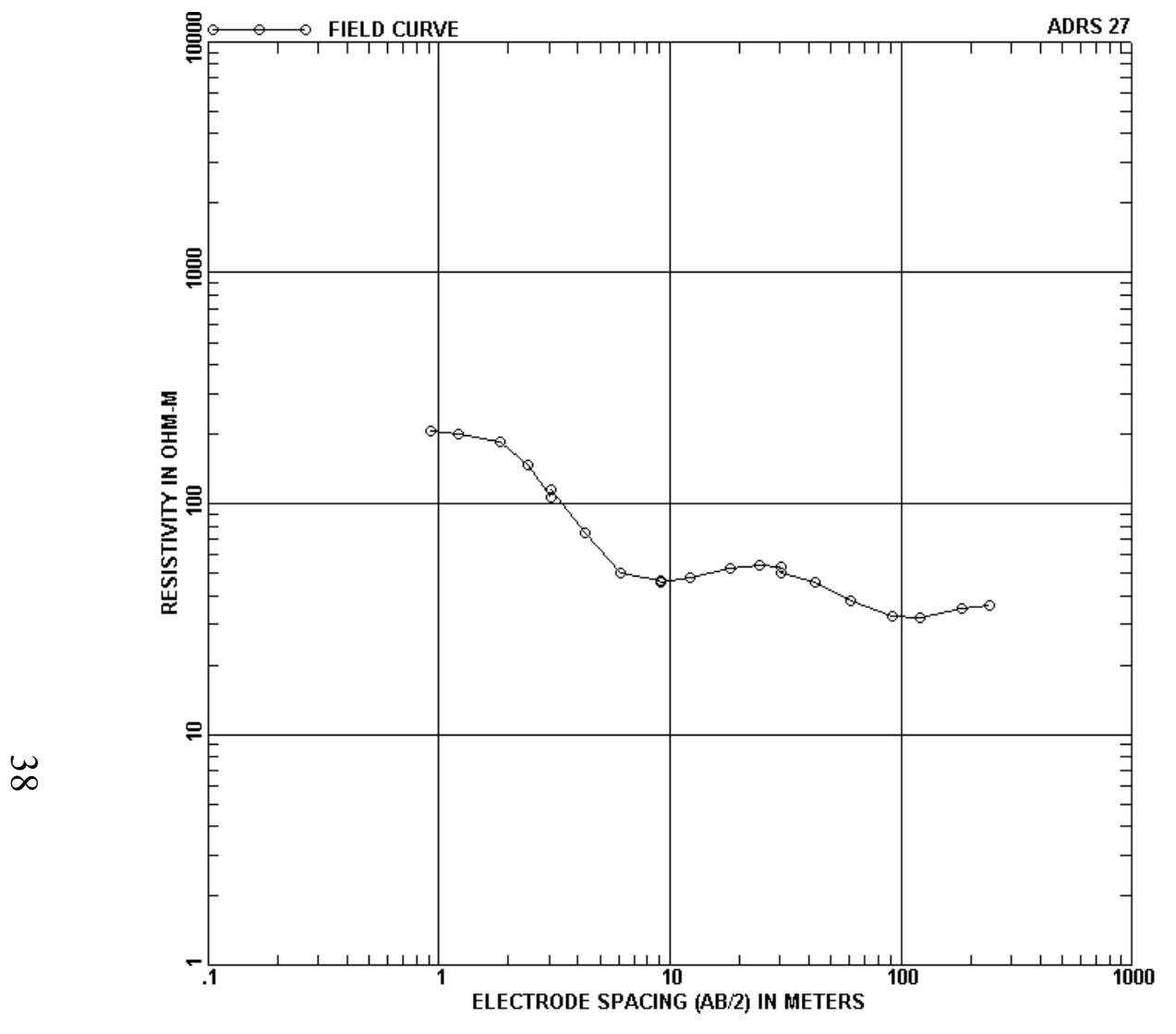

$A B / 2, m$ ( ft ) App. Res. $A B / 2, m$ ( ft ) App. Res.

\begin{tabular}{|c|c|c|}
\hline 0.91 & $3.00)$ & 207.00 \\
\hline 1.22 & $4.00)$ & 200.00 \\
\hline 1.83 & $6.00)$ & 185.00 \\
\hline 2.44 & $8.00)$ & 146.00 \\
\hline 3.05 & $10.00)$ & 107.00 \\
\hline 3.05 & $10.00)$ & 115.00 \\
\hline 4.27 & $14.00)$ & 75.00 \\
\hline 6.10 & $20.00)$ & 50.20 \\
\hline 9.14 & $30.00)$ & 46.00 \\
\hline 9.14 & $30.00)$ & 45.30 \\
\hline 2.19 & $40.00)$ & 48.00 \\
\hline
\end{tabular}

52.10
54.00
53.50
49.90
45.40
37.80
32.50
32.60
31.80
35.20
36.40

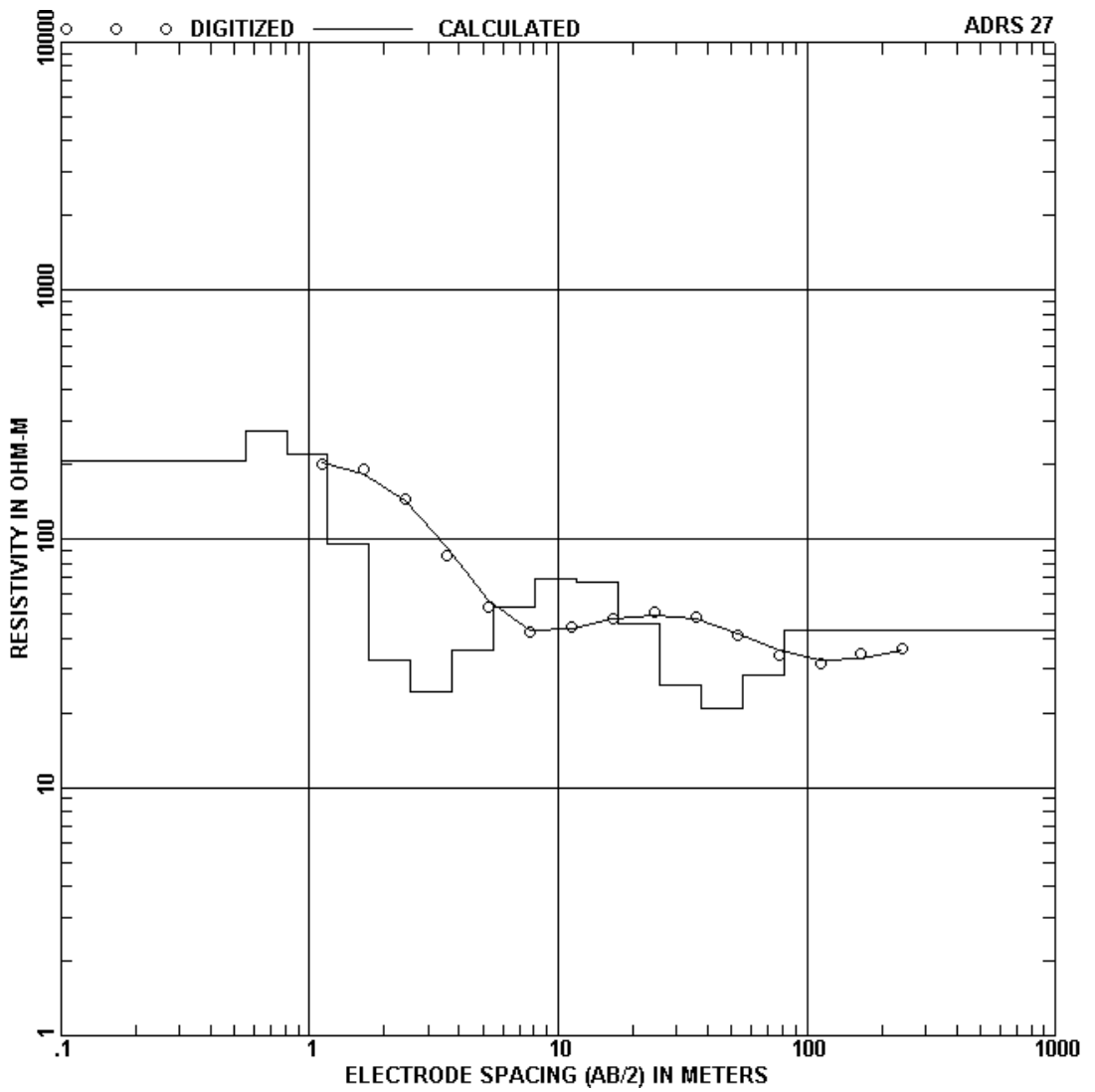

DEPTH, m ( ft ) RESIS. DEPTH, m ( ft ) RESIS.

$\begin{array}{rlr}0.55 & ( & 1.80) \\ 0.81 & ( & 2.65) \\ 1.19 & ( & 3.89) \\ 1.74 & ( & 5.71) \\ 2.55 & ( & 8.38) \\ 3.75 & ( & 12.29) \\ 5.50 & ( & 18.05)\end{array}$

206.07

271.30

220.19

95.66

32.55

24.44

35.88
53.59

69.07

67.05

45.30

25.82

20.97

28.25

43.09 


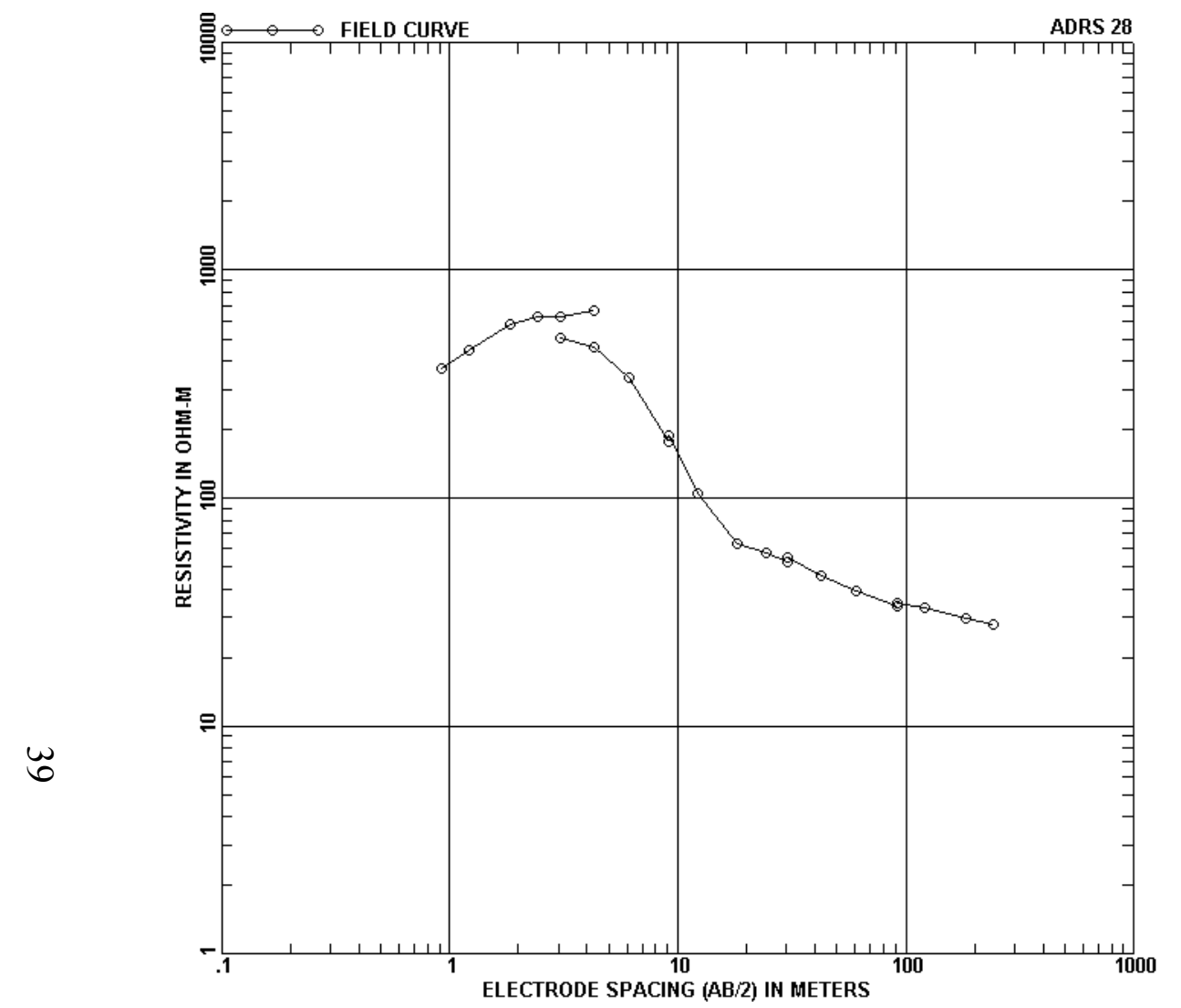

$A B / 2, m$ ( ft ) App. Res. $A B / 2, m$ ( ft ) App. Res.

\begin{tabular}{|c|c|c|}
\hline 0.91 & $3.00)$ & 369.00 \\
\hline 1.22 & $4.00)$ & 445.00 \\
\hline 1.83 & $6.00)$ & 576.00 \\
\hline 2.44 & $8.00)$ & 621.00 \\
\hline 3.05 & $10.00)$ & 629.00 \\
\hline 4.27 & $14.00)$ & 665.00 \\
\hline 3.05 & $10.00)$ & 504.00 \\
\hline 4.27 & $14.00)$ & 460.00 \\
\hline 6.10 & $20.00)$ & 339.00 \\
\hline 9.14 & $30.00)$ & 178.00 \\
\hline 9.14 & $30.00)$ & 189.00 \\
\hline
\end{tabular}

\begin{tabular}{|c|c|c|}
\hline 12.19 & $40.00)$ & 105.00 \\
\hline 18.29 & $60.00)$ & 63.30 \\
\hline 24.38 & $80.00)$ & 57.80 \\
\hline 30.48 & $100.00)$ & 52.20 \\
\hline 30.48 & $100.00)$ & 54.50 \\
\hline 42.67 & $140.00)$ & 45.70 \\
\hline 60.96 & $200.00)$ & 39.10 \\
\hline 91.44 & $300.00)$ & 33.40 \\
\hline 91.44 & $300.00)$ & 34.50 \\
\hline 121.92 & $400.00)$ & 32.90 \\
\hline 182.88 & $600.00)$ & 29.60 \\
\hline 243.84 & $800.00)$ & 27.90 \\
\hline
\end{tabular}

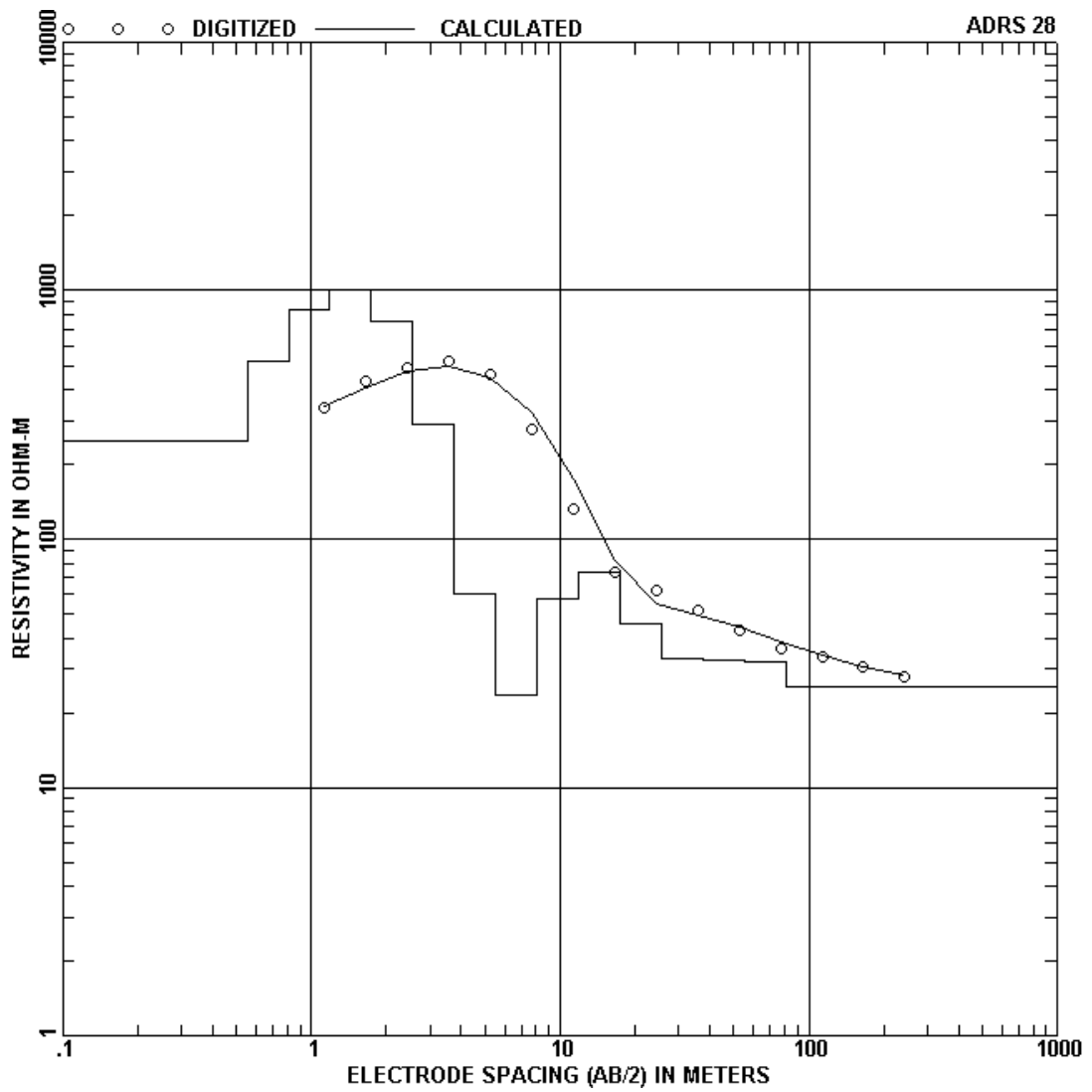

DEPTH, m ( ft ) RESIS. DEPTH, m ( ft ) RESIS.

\begin{tabular}{|c|c|c|c|c|c|}
\hline 0.55 & $1.80)$ & 248.61 & 8.07 & $26.49)$ & 23.60 \\
\hline 0.81 & $2.65)$ & 516.57 & 11.85 & $38.88)$ & 57.29 \\
\hline 1.19 & $3.89)$ & 839.63 & 17.39 & $57.07)$ & 73.03 \\
\hline 1.74 & $5.71)$ & 1002.71 & 25.53 & $83.76)$ & 45.78 \\
\hline 2.55 & $8.38)$ & 753.23 & 37.47 & $122.95)$ & 32.91 \\
\hline 3.75 & $12.29)$ & 290.52 & 55.01 & $180.47)$ & 32.67 \\
\hline 5.50 & $18.05)$ & 60.38 & $\begin{array}{r}80.74 \\
99999.00\end{array}$ & $\begin{array}{r}(264.89) \\
(99999.00)\end{array}$ & $\begin{array}{l}31.92 \\
25.27\end{array}$ \\
\hline
\end{tabular}



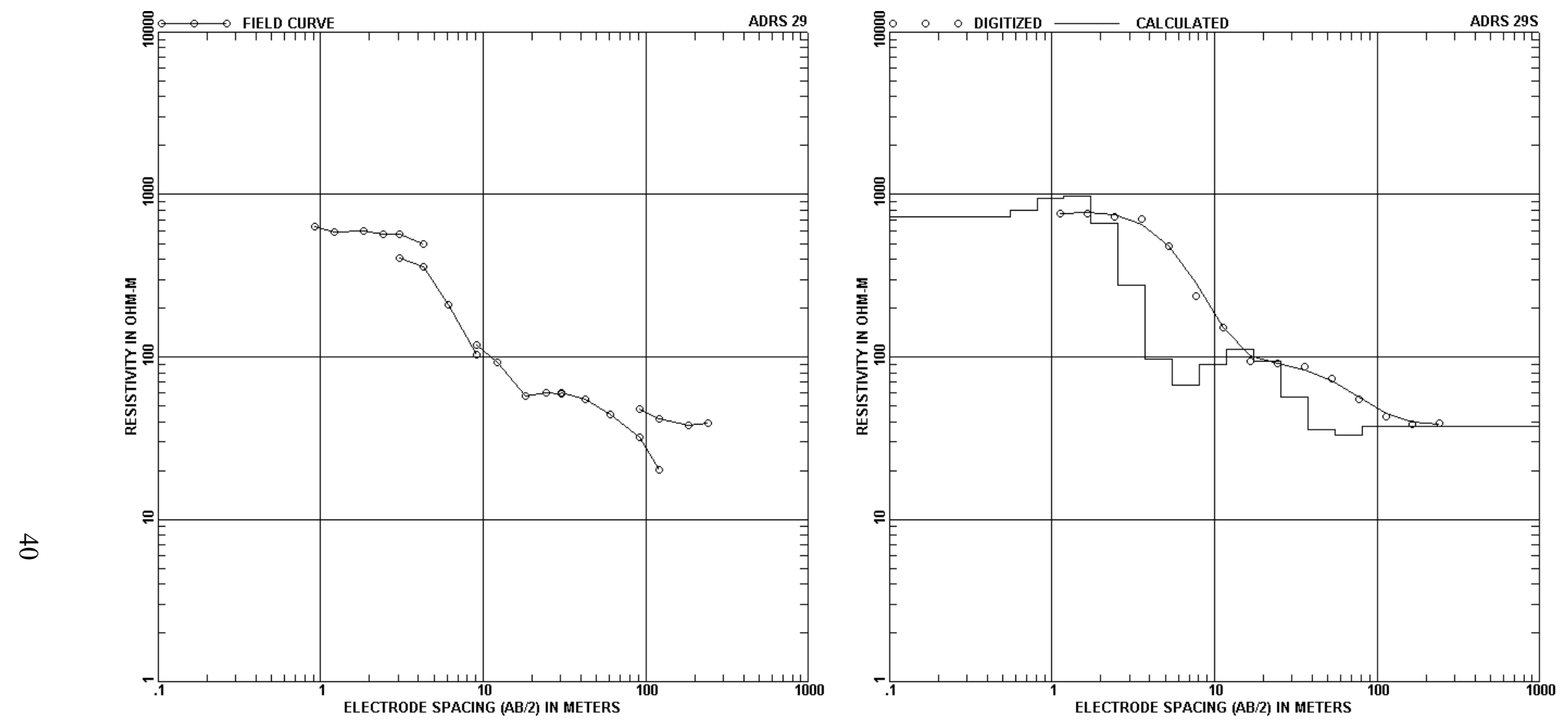

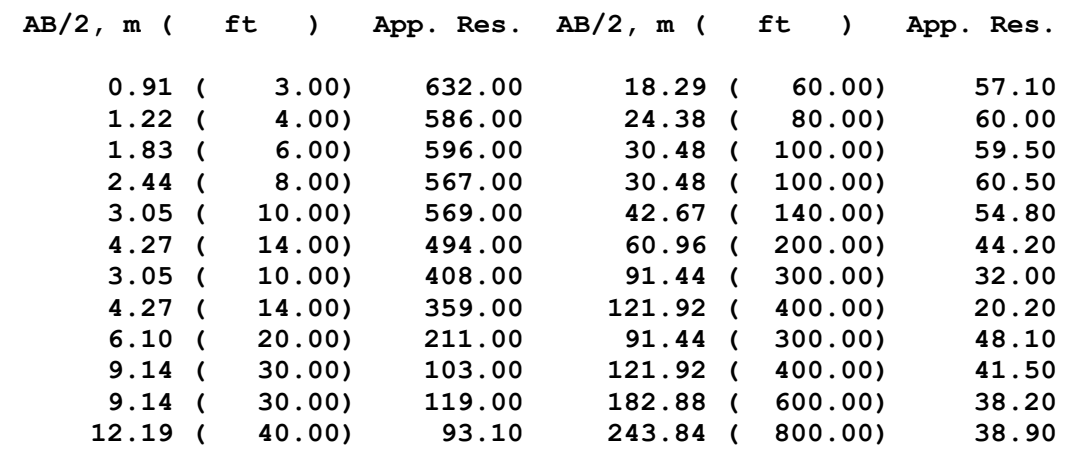

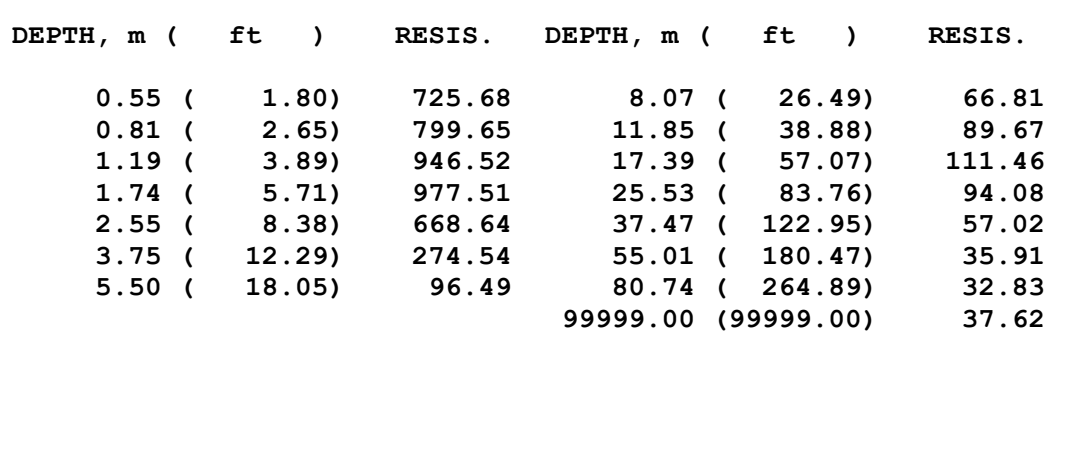




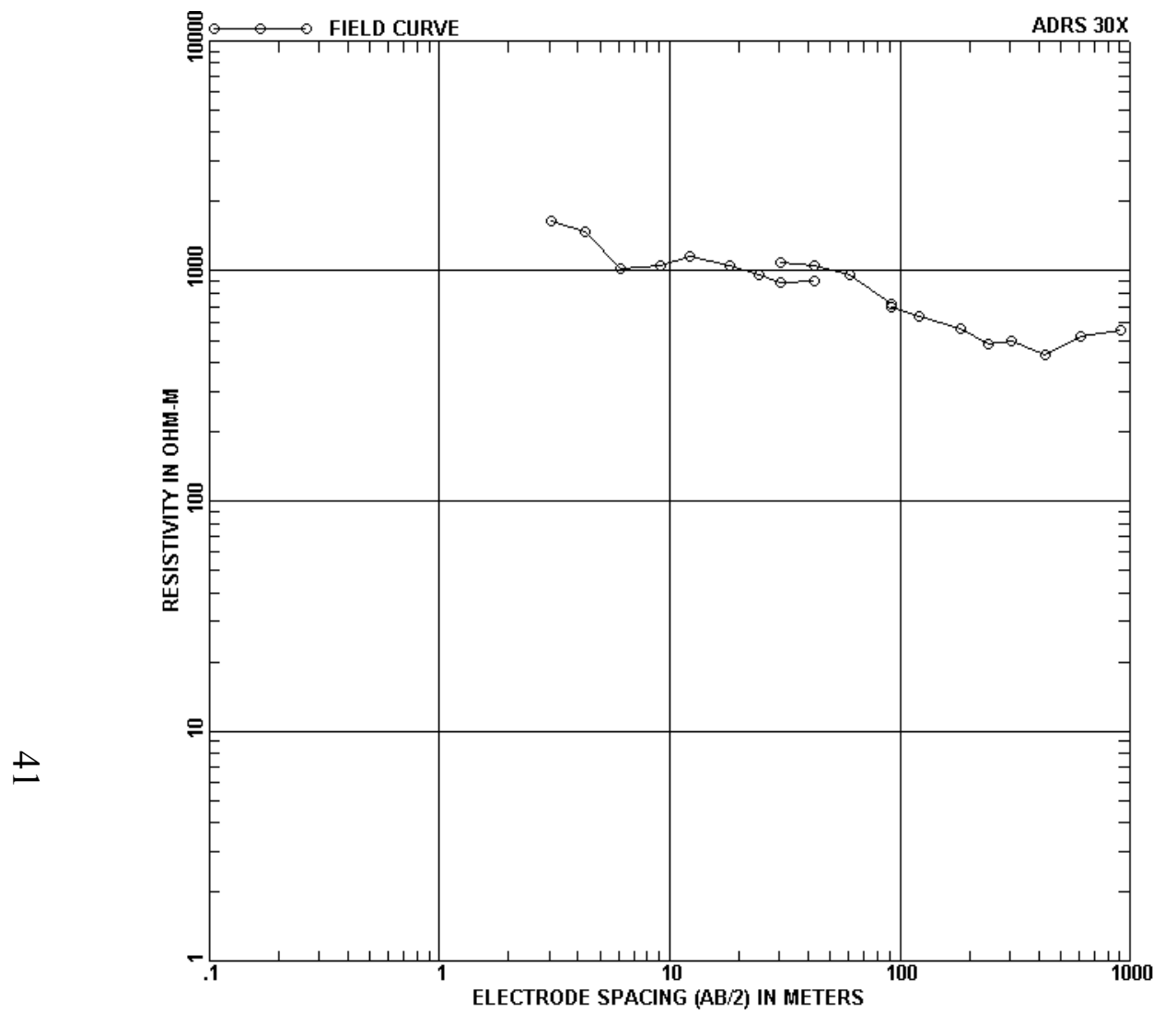

$A B / 2, m(f t)$ App. Res. $A B / 2, m$ ( ft ) App. Res.

\begin{tabular}{|c|c|c|}
\hline 05 & $10.00)$ & 1650.00 \\
\hline 4.27 & $14.00)$ & 1470.0 \\
\hline 6.10 & $20.00)$ & 5. \\
\hline 9.14 & 30 . & 1060.00 \\
\hline 12.19 & 40. & 1150.00 \\
\hline 8.29 & 0. & 1050.00 \\
\hline 24.38 & 80. & 960.00 \\
\hline 30.48 & 100. & 890.00 \\
\hline 42.67 & 140. & 910.00 \\
\hline 30.4 & 100. & 1080.00 \\
\hline 42.67 & $140.00)$ & 1060.0 \\
\hline
\end{tabular}

$\begin{array}{rrr}60.96 & (200.00) & 960.00 \\ 91.44 & (300.00) & 720.00 \\ 91.44 & (300.00) & 700.00 \\ 121.92 & ((400.00) & 630.00 \\ 182.88 & (600.00) & 560.00 \\ 243.84 & (800.00) & 480.00 \\ 304.80 & (1000.00) & 500.00 \\ 304.80(1000.00) & 500.00 \\ 426.72(1400.00) & 430.00 \\ 609.60(2000.00) & 520.00 \\ 914.40(3000.00) & 550.00\end{array}$

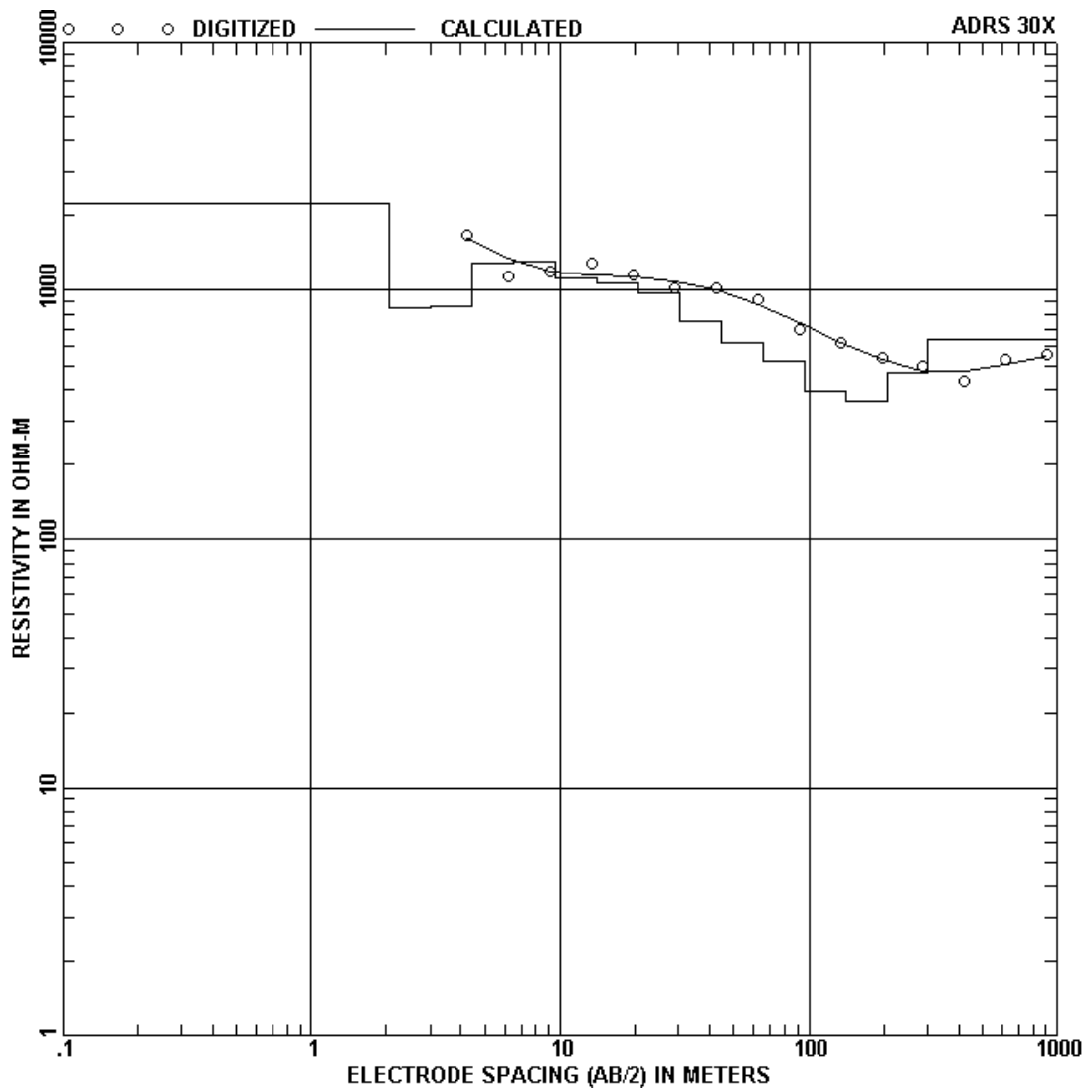

DEPTH, $m$ ( ft ) RESIS. DEPTH, $m$ ( ft ) RESIS.

\begin{tabular}{|c|c|c|c|c|c|}
\hline 2.06 & $6.77)$ & 2255.45 & 30.28 & $99.33)$ & 975.80 \\
\hline 3.03 & 9.93) & 847.23 & 44.44 & $145.80)$ & 756.34 \\
\hline 4.44 & $14.58)$ & 859.46 & 65.23 & 214.01) & 611.74 \\
\hline 6.52 & $21.40)$ & 1278.99 & 95.74 & $314.12)$ & 515.78 \\
\hline 9.57 & $31.41)$ & 1306.32 & 140.53 & $461.06)$ & 391.62 \\
\hline 14.05 & $46.11)$ & 1130.57 & 206.27 & $676.74)$ & 360.73 \\
\hline 20.63 & $67.67)$ & 1079.34 & $\begin{array}{r}302.77 \\
99999.00\end{array}$ & $\begin{array}{r}(993.32) \\
(99999.00)\end{array}$ & $\begin{array}{l}468.79 \\
631.29\end{array}$ \\
\hline
\end{tabular}



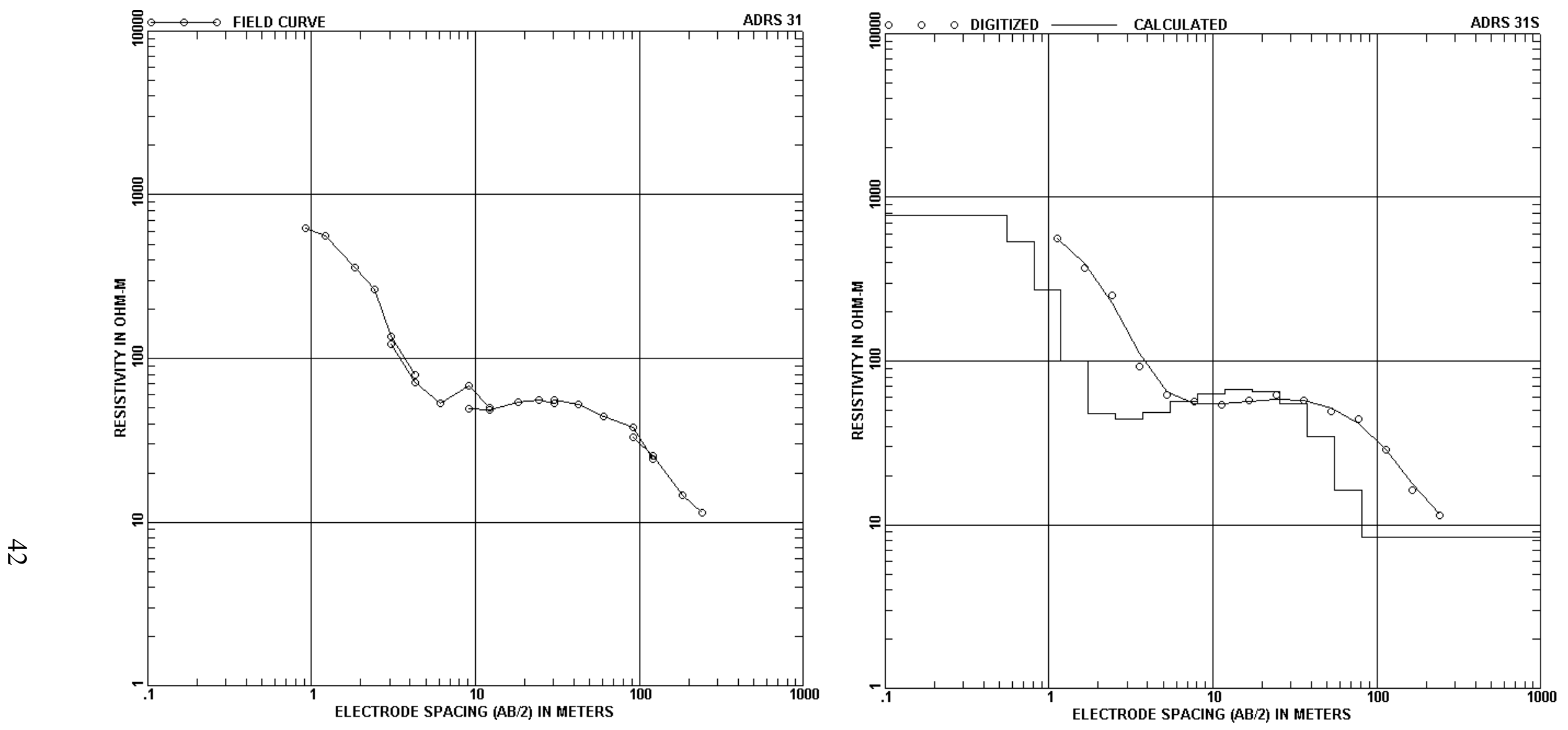

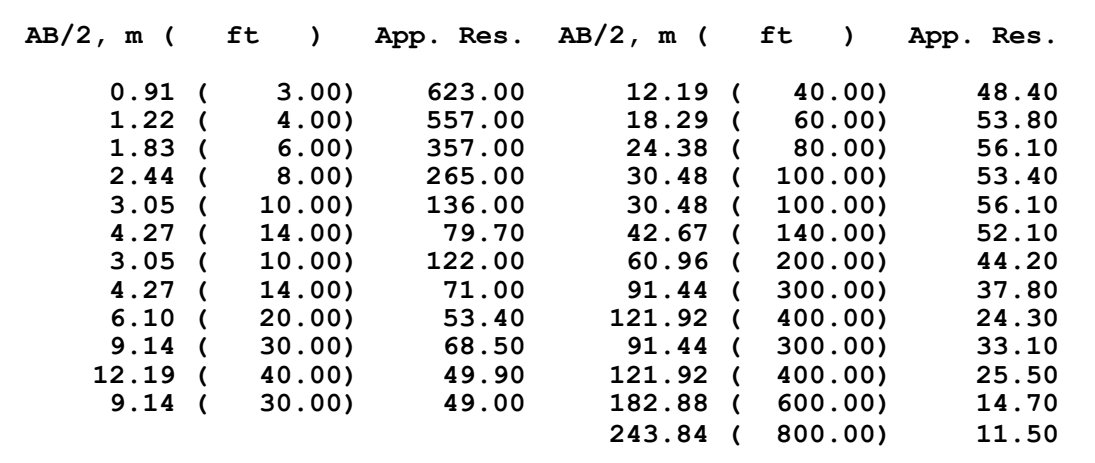

DEPTH, m ( ft )

$\begin{array}{rlr}0.55 & ( & 1.80) \\ 0.81 & ( & 2.65) \\ 1.19 & ( & 3.89) \\ 1.74 & ( & 5.71) \\ 2.55 & ( & 8.38) \\ 3.75 & ( & 12.29) \\ 5.50 & ( & 18.05)\end{array}$

RESIS.

774.33

534.72

534.72
273.30

100.15

48.04

43.91

48.52
DEPTH， m ( ft

\section{$8.07(26.49)$}

$11.85(38.88)$

17.39 ( 57.07)

$25.53(83.76)$

$37.47(122.95)$

55.01 ( 180.47)

80.74 ( 264.89) $99999.00 \quad(99999.00)$
RESIS.

56.33

63.49

66.78

65.33

55.10

34.68

16.28

列



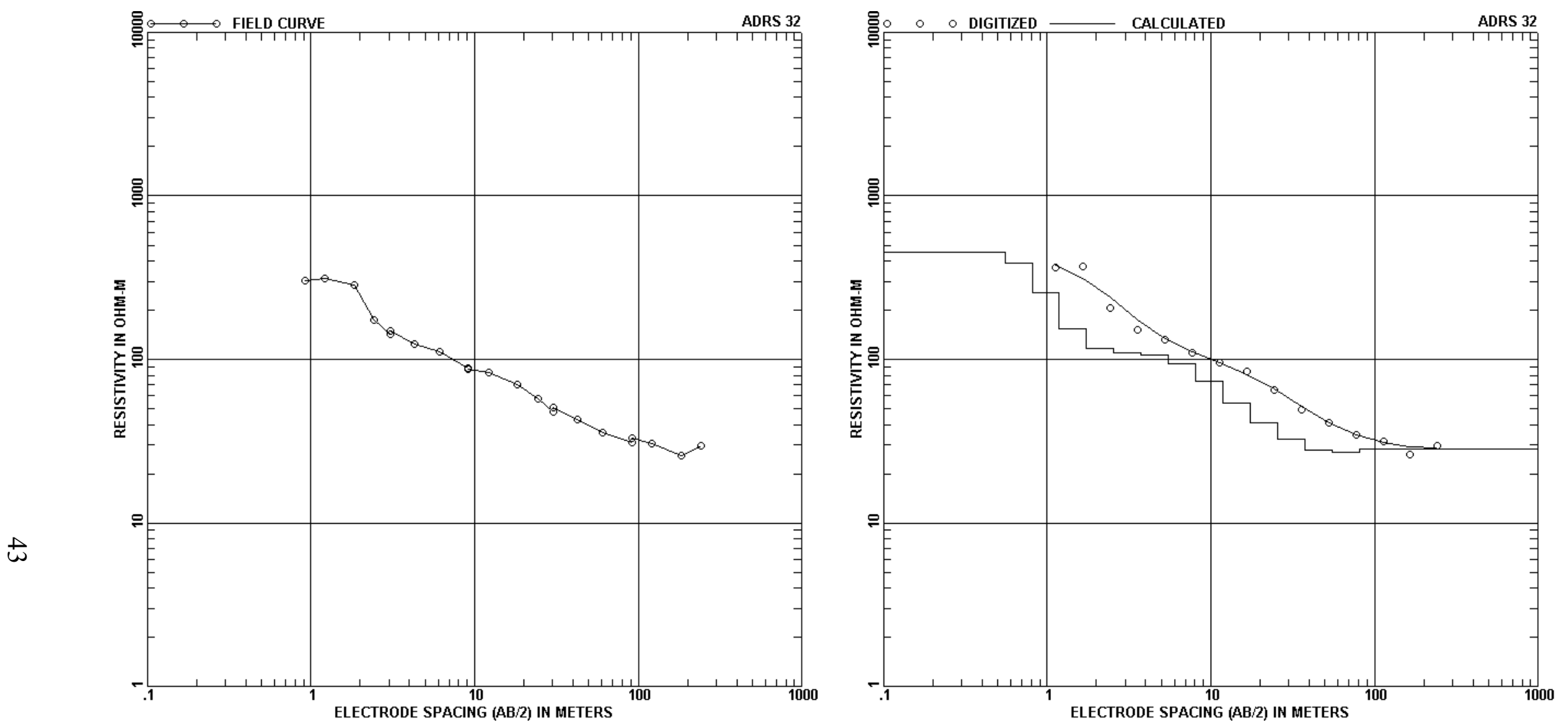

$A B / 2$, $m$ ( ft ) App. Res. $A B / 2$, $m$ ( ft ) App. Res.

DEPTH, $m($ ft )

RESIS .

DEPTH, m ( ft )

RESIS.

\begin{tabular}{|c|c|c|}
\hline 0.91 & $3.00)$ & 301.00 \\
\hline 1.22 & $4.00)$ & 311.00 \\
\hline 1.83 & $6.00)$ & 285.00 \\
\hline 2.44 & $8.00)$ & 173.00 \\
\hline 3.05 & $10.00)$ & 142.00 \\
\hline 3.05 & $10.00)$ & 150.00 \\
\hline 4.27 & $14.00)$ & 124.00 \\
\hline 6.10 & $20.00)$ & 112.00 \\
\hline 9.14 & $30.00)$ & 88.40 \\
\hline 9.14 & $30.00)$ & 87.50 \\
\hline 12.19 & $40.00)$ & 82.90 \\
\hline
\end{tabular}

$18.29(60.00) \quad 70.70$ $24.38(80.00) \quad 57.20$ $30.48(100.00) \quad 47.70$ $30.48(100.00) \quad 51.00$ $42.67(140.00) \quad 43.00$ $60.96(200.00) \quad 35.50$ $91.44(300.00) \quad 30.90$ $91.44(300.00)$ $91.44(300.00) \quad 32.90$ $\begin{array}{ll}121.92(400.00) & 30.80\end{array}$ $\begin{array}{lll}182.88 & (600.00) & 26.00 \\ 243.84(800.00) & 29.50\end{array}$

\begin{tabular}{|c|c|c|}
\hline 0.55 & $1.80)$ & 450.43 \\
\hline 0.81 & $2.65)$ & 388.56 \\
\hline 1.19 & $3.89)$ & 256.06 \\
\hline 1.74 & $5.71)$ & 154.59 \\
\hline 2.55 & $8.38)$ & 117.02 \\
\hline 3.75 & $12.29)$ & 109.65 \\
\hline 5.50 & $18.05)$ & 105.69 \\
\hline
\end{tabular}

\begin{tabular}{|c|c|}
\hline 8.07 & $26.49)$ \\
\hline 11.85 & $38.88)$ \\
\hline 17.39 & $57.07)$ \\
\hline 25.53 & $83.76)$ \\
\hline 37.47 & $122.95)$ \\
\hline 55.01 & $180.47)$ \\
\hline 80.74 & $264.89)$ \\
\hline
\end{tabular}

93.74

73.44

54.16

40.94

32.64

27.97 26.90 

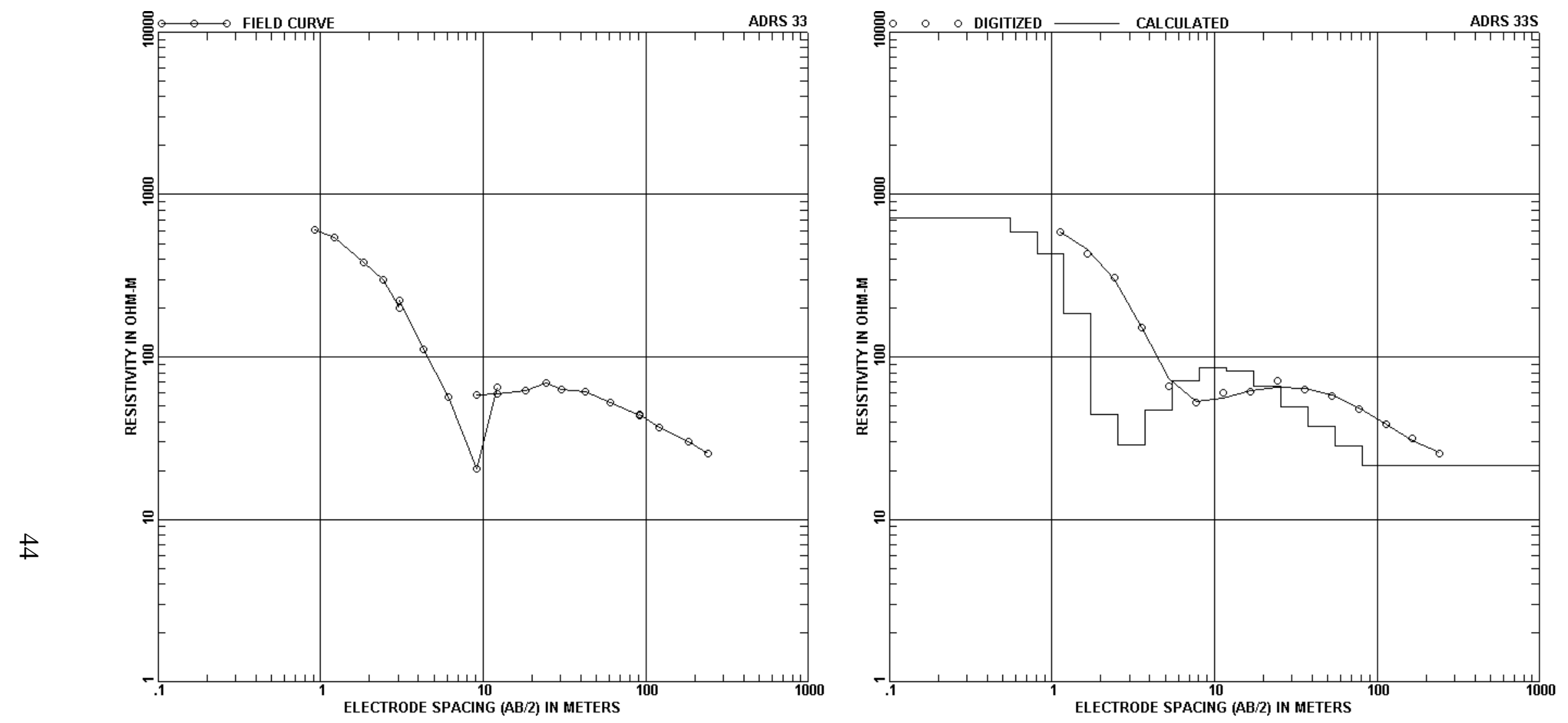

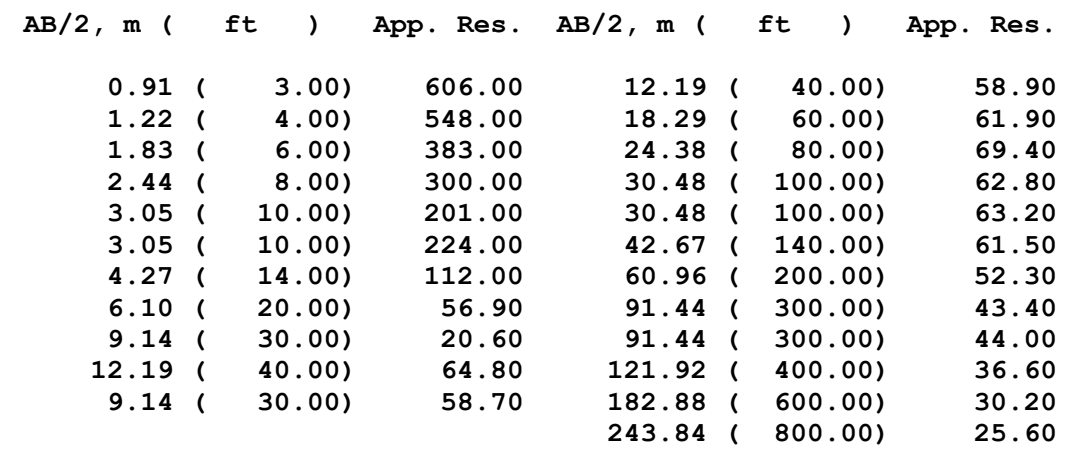

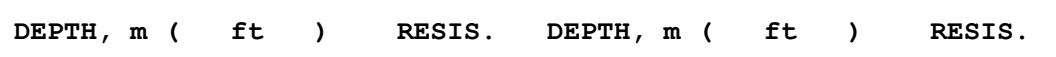

$\begin{array}{rrrrrr}0.55( & 1.80) & 719.65 & 8.07( & 26.49) & 70.98 \\ 0.81( & 2.65) & 590.04 & 11.85( & 38.88) & 85.77 \\ 1.19( & 3.89) & 431.46 & 17.39( & 57.07) & 82.12 \\ 1.74( & 5.71) & 186.55 & 25.53( & 83.76) & 66.15 \\ 2.55( & 8.38) & 44.59 & 37.47( & 122.95) & 49.55 \\ 3.75( & 12.29) & 28.70 & 55.01(180.47) & 37.44 \\ 5.50( & 18.05) & 47.18 & 80.74(264.89) & 28.41 \\ & & & 99999.00(99999.00) & 21.58\end{array}$




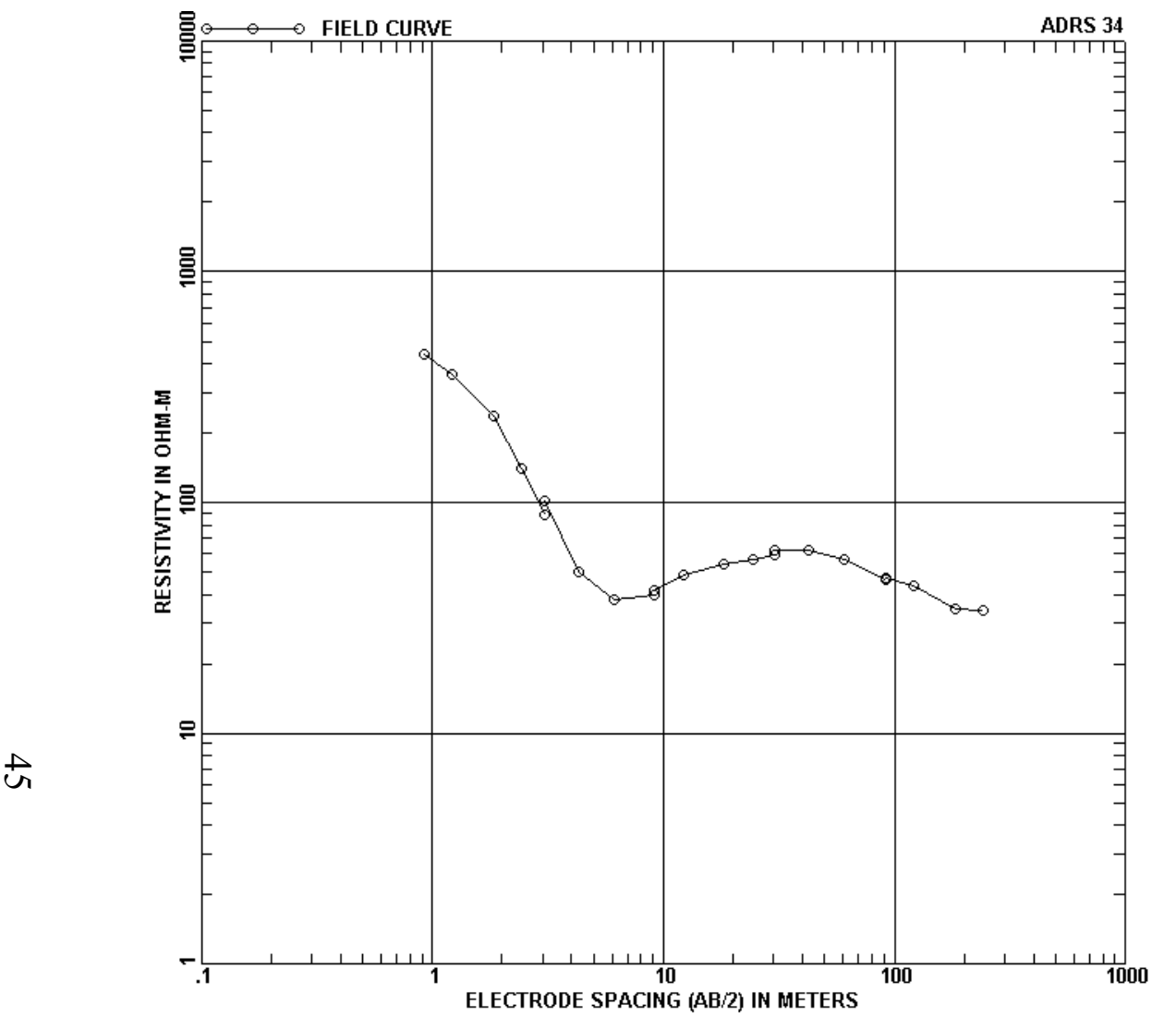

$A B / 2, m(f t)$ App. Res. $A B / 2, m$ ( ft ) App. Res.

\begin{tabular}{|c|c|c|}
\hline 0.91 & $3.00)$ & 438.00 \\
\hline 1.22 & $4.00)$ & 360.00 \\
\hline 1.83 & $6.00)$ & 236.00 \\
\hline 2.44 & $8.00)$ & 140.00 \\
\hline 3.05 & $10.00)$ & 88.20 \\
\hline 3.05 & $10.00)$ & 102.00 \\
\hline 4.27 & $14.00)$ & 50.40 \\
\hline 6.10 & $20.00)$ & 37.80 \\
\hline 9.14 & $30.00)$ & 39.90 \\
\hline 9.14 & $30.00)$ & 41.90 \\
\hline 2.19 & $40.00)$ & 48.20 \\
\hline
\end{tabular}

53.80
56.70
59.50
61.90
62.00
56.80
46.40
46.80
43.90
34.50
34.00

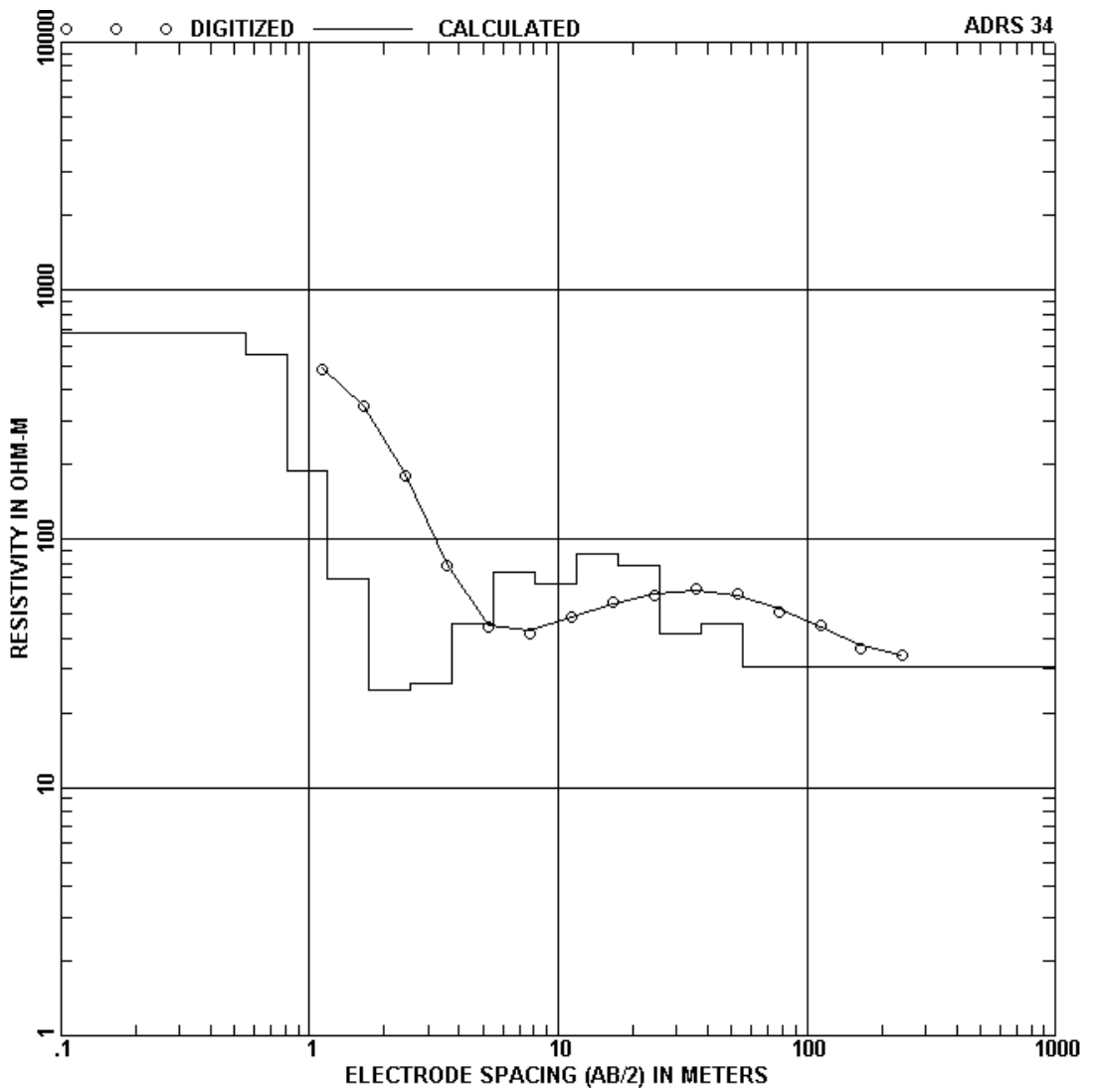

DEPTH, m ( ft ) RESIS. DEPTH, m ( ft ) RESIS.

$\begin{array}{rlr}0.55 & ( & 1.80) \\ 0.81 & ( & 2.65) \\ 1.19 & ( & 3.89) \\ 1.74 & ( & 5.71) \\ 2.55 & ( & 8.38) \\ 3.75 & ( & 12.29) \\ 5.50 & ( & 18.05)\end{array}$

671.98

555.22

189.23

68.81

26.28

45.68

\begin{tabular}{|c|c|}
\hline 8.07 & $26.49)$ \\
\hline 11.85 & $38.88)$ \\
\hline 17.39 & $57.07)$ \\
\hline 25.53 & $83.76)$ \\
\hline 37.47 & $122.95)$ \\
\hline 55.01 & $180.47)$ \\
\hline 80.74 & $264.89)$ \\
\hline 99.00 & 99999.00) \\
\hline
\end{tabular}

73.58

66.18

87.68

77.76

41.69

45.80

30.53

30.65 

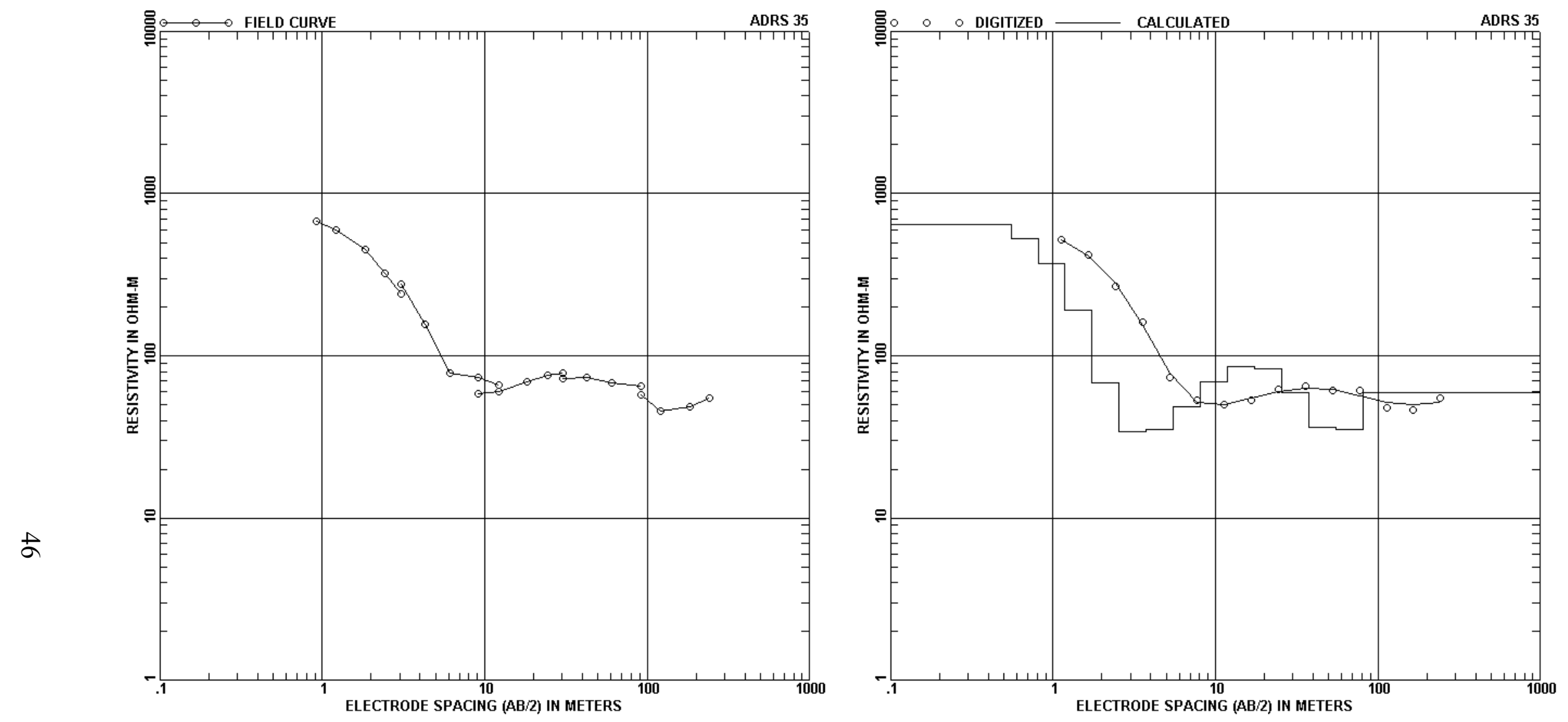

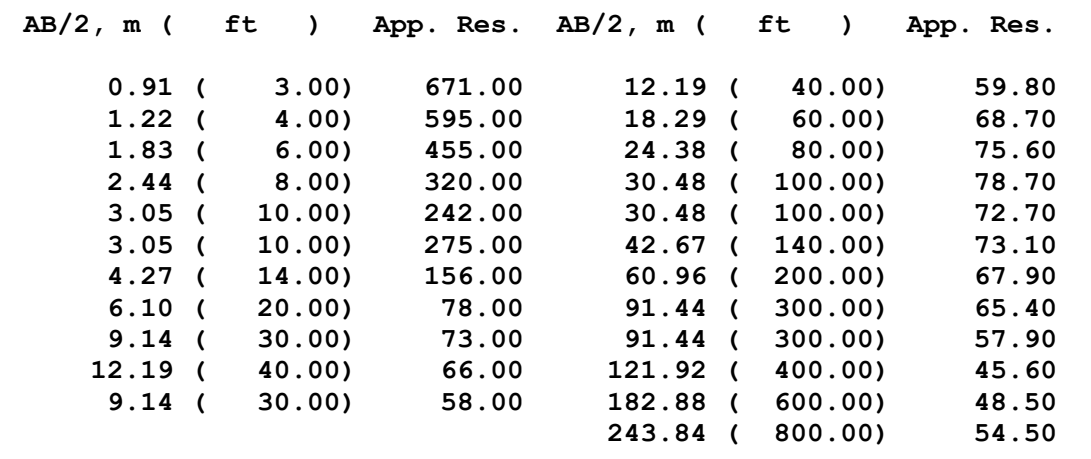

DEPTH, $m$ ( ft ) RESIS. DEPTH, $m($ ft ) RESIS.

\begin{tabular}{|c|c|c|}
\hline 0.55 & $1.80)$ & 641.84 \\
\hline 0.81 & $2.65)$ & 526.42 \\
\hline 1.19 & $3.89)$ & 368.19 \\
\hline 1.74 & $5.71)$ & 190.09 \\
\hline 2.55 & $8.38)$ & 67.65 \\
\hline 3.75 & $12.29)$ & 34.24 \\
\hline 5.50 & $18.05)$ & 35.13 \\
\hline
\end{tabular}

$\begin{array}{rlr}8.07 & ( & 26.49) \\ 11.85 & ( & 38.88) \\ 17.39 & ( & 57.07) \\ 25.53 & ( & 83.76) \\ 37.47 & ( & 122.95) \\ 55.01 & ( & 180.47) \\ 80.74 & ( & 264.89)\end{array}$

48.56

69.03

85.37

83.28

58.86

36.33

35.06

59.58 

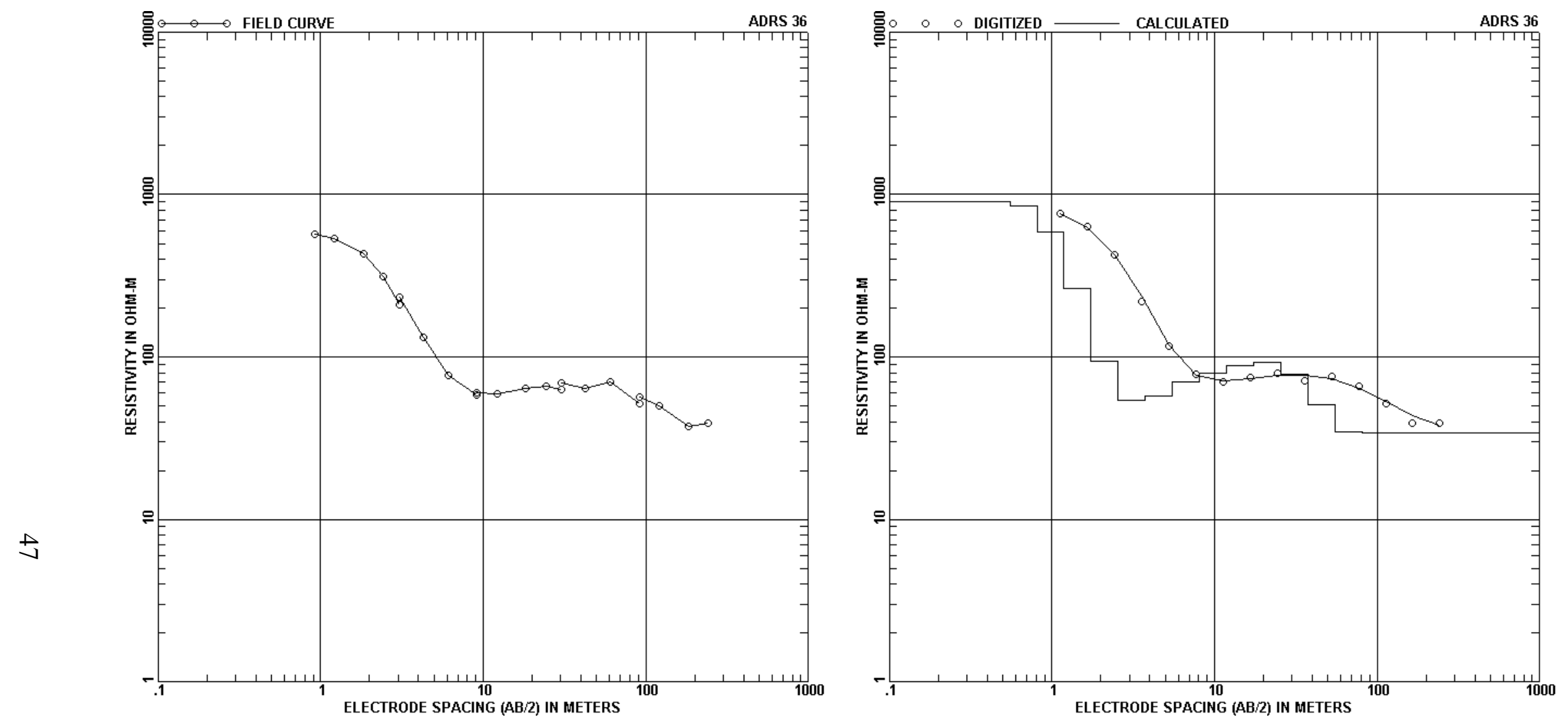

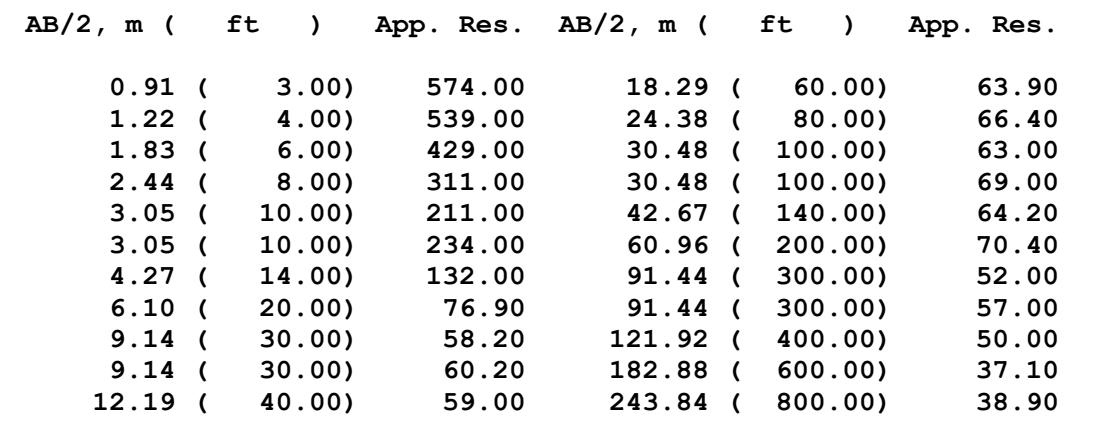

\begin{tabular}{|c|c|c|c|c|c|}
\hline DEPTH， m ( & ft , & RESIS. & DEPTH， m & ft & RESIS. \\
\hline 0.551 & $1.80)$ & 899.93 & 8.07 & $26.49)$ & 69.83 \\
\hline $0.81 i$ & $2.65)$ & 856.46 & 11.85 & $38.88)$ & 79.41 \\
\hline 1.19 i & $3.89)$ & 587.29 & 17.39 & $57.07)$ & 88.31 \\
\hline $1.74 i$ & $5.71)$ & 262.51 & 25.53 & $83.76)$ & 92.66 \\
\hline $2.55 i$ & $8.38)$ & 94.63 & 37.47 & $122.95)$ & 77.82 \\
\hline $3.75 i$ & $12.29)$ & 54.25 & 55.01 & $180.47)$ & 50.50 \\
\hline $5.50 i$ & $18.05)$ & 57.55 & 80.74 & (264.89) & 34.42 \\
\hline & & & 99999.00 & $(99999.00)$ & 33.84 \\
\hline
\end{tabular}



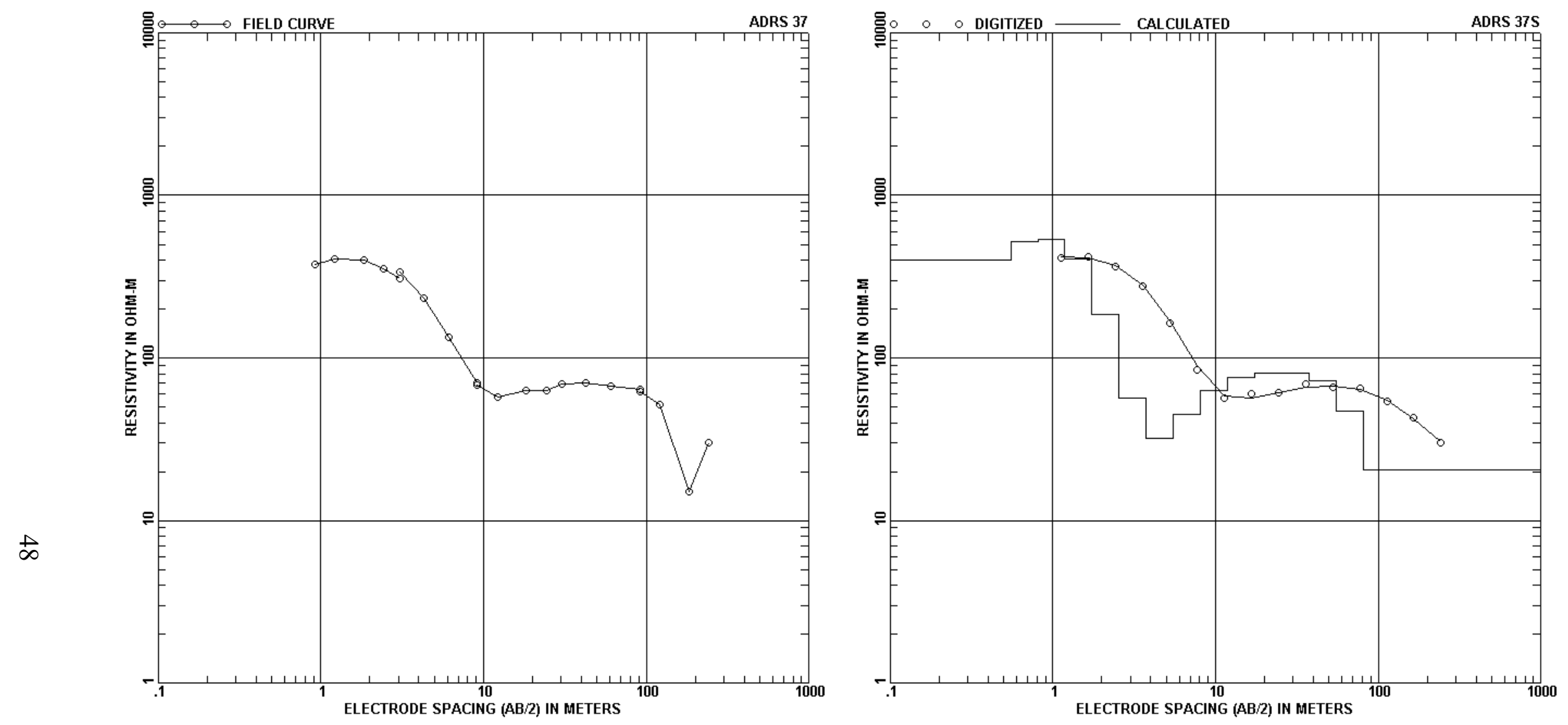

$A B / 2$, $m$ ( ft ) App. Res. $A B / 2$, $m$ ( ft ) App. Res.

DEPTH, $m$ ( ft ) RESIS. DEPTH, m ( ft ) RESIS.

\begin{tabular}{|c|c|c|c|c|c|}
\hline 0.91 & $3.00)$ & 378.00 & 18.29 & $60.00)$ & 62.80 \\
\hline 1.22 & $4.00)$ & 405.00 & 24.38 & $80.00)$ & 62.60 \\
\hline 1.83 & $6.00)$ & 398.00 & 30.48 & $(100.00)$ & 68.80 \\
\hline 2.44 & $8.00)$ & 352.00 & 30.48 & $(100.00)$ & 69.50 \\
\hline 3.05 & $10.00)$ & 308.00 & 42.67 & $(140.00)$ & 70.70 \\
\hline 3.05 & $10.00)$ & 336.00 & 60.96 & $200.00)$ & 67.30 \\
\hline 4.27 & $14.00)$ & 235.00 & 91.44 & $300.00)$ & 64.40 \\
\hline 6.10 & $20.00)$ & 135.00 & 91.44 & $300.00)$ & 62.00 \\
\hline 9.14 & $30.00)$ & 69.80 & 121.92 & $(400.00)$ & 51.30 \\
\hline 9.14 & $30.00)$ & 68.30 & 182.88 & $600.00)$ & 15.00 \\
\hline 12.19 & $40.00)$ & 57.60 & 243.84 & $800.00)$ & 30.10 \\
\hline
\end{tabular}

\begin{tabular}{|c|c|c|}
\hline 0.55 & $1.80)$ & 399.66 \\
\hline 0.81 & $2.65)$ & 516.77 \\
\hline 1.19 & $3.89)$ & 538.59 \\
\hline 1.74 & $5.71)$ & 404.40 \\
\hline 2.55 & $8.38)$ & 186.08 \\
\hline 3.75 & $12.29)$ & 56.24 \\
\hline 5.50 & $18.05)$ & 32.02 \\
\hline
\end{tabular}

$\begin{array}{rlr}8.07 & ( & 26.49) \\ 11.85 & ( & 38.88) \\ 17.39 & ( & 57.07) \\ 25.53 & ( & 83.76) \\ 37.47 & ( & 122.95) \\ 55.01 & ( & 180.47) \\ 80.74 & ( & 264.89)\end{array}$

44.82

62.89

75.72

81.03

80.92

71.86 $99999.00(99999.00) \quad 20.35$ 

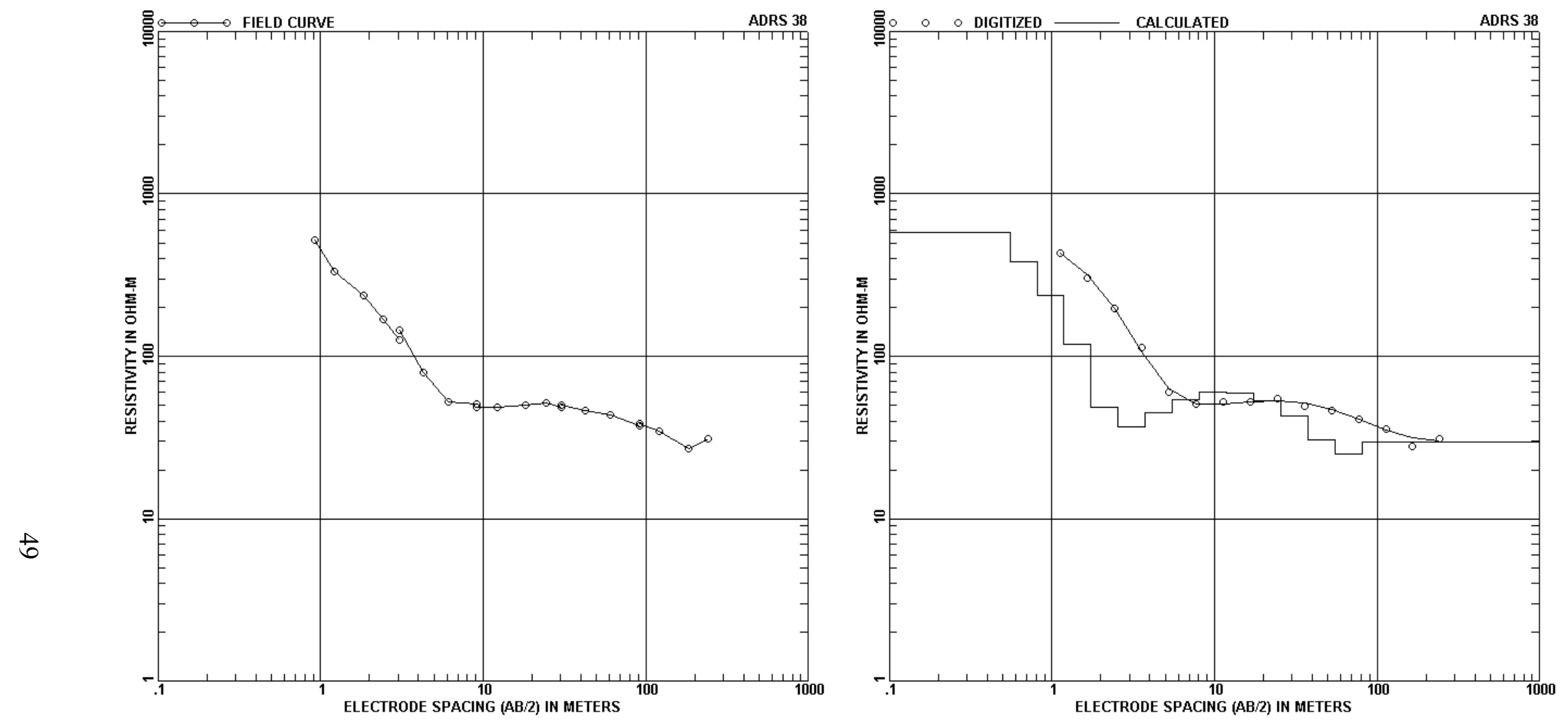

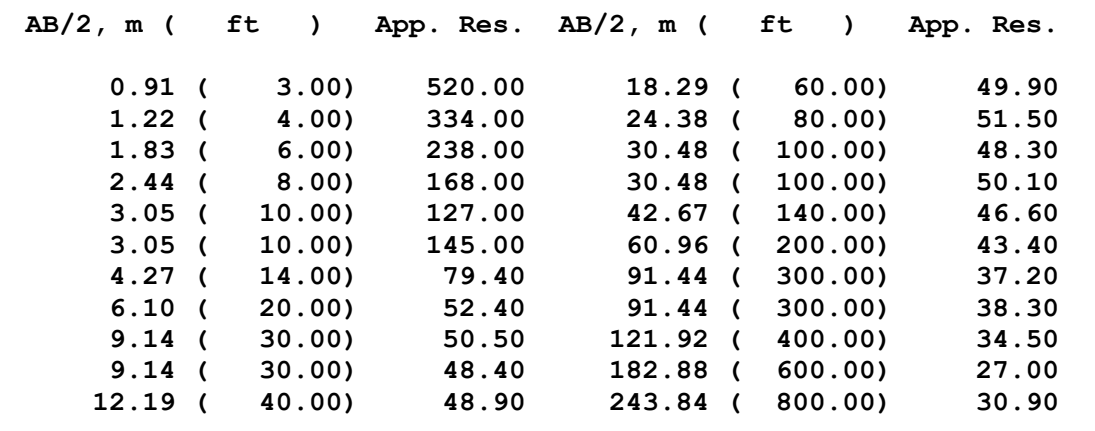

\begin{tabular}{|c|c|c|c|c|c|}
\hline DEPTH， m ( & ft, & RESIS. & DEPTH, m & ft & RESIS. \\
\hline 0.55 & $1.80)$ & 581.84 & 8.07 & $26.49)$ & 54.37 \\
\hline 0.81 & $2.65)$ & 379.32 & 11.85 & $38.88)$ & 59.75 \\
\hline 1.19 & $3.89)$ & 236.06 & 17.39 & $57.07)$ & 59.12 \\
\hline 1.74 & $5.71)$ & 118.76 & 25.53 & $83.76)$ & 53.60 \\
\hline 2.55 & $8.38)$ & 48.78 & 37.47 & $122.95)$ & 42.89 \\
\hline 3.75 & $12.29)$ & 36.70 & 55.01 & $(180.47)$ & 30.49 \\
\hline 5.50 & $18.05)$ & 44.73 & 80.74 & ( 264.89) & 25.17 \\
\hline & & & 99999.00 & $(99999.00)$ & 29.43 \\
\hline
\end{tabular}




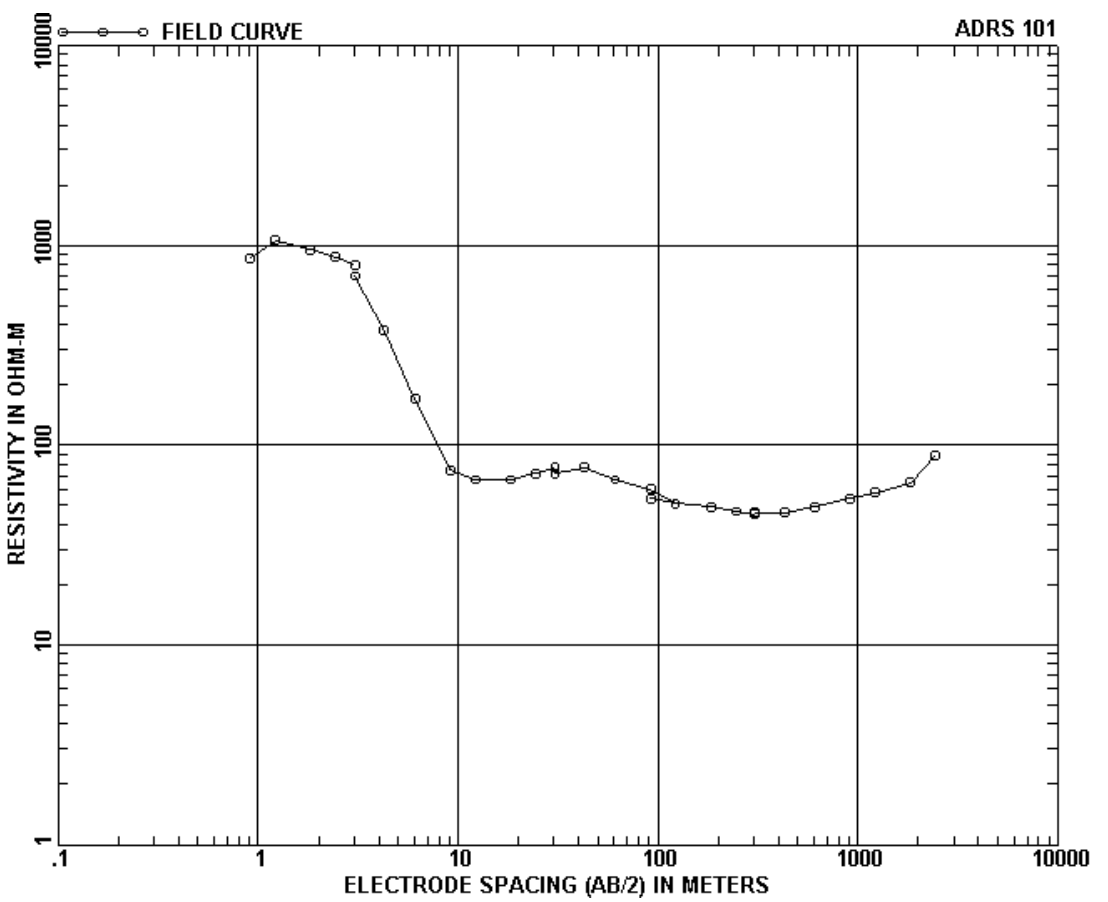

$A B / 2$, m (ft ) App. Res. $A B / 2$, m ( ft ) App. Res.

\begin{tabular}{|c|c|c|}
\hline 0.91 & $3.00)$ & 860.00 \\
\hline 1.22 & $4.00)$ & 1060.00 \\
\hline 1.83 & $6.00)$ & 950.00 \\
\hline 2.44 & $8.00)$ & 870.00 \\
\hline 3.05 & $10.00)$ & 798.00 \\
\hline 3.05 & $10.00)$ & 702.00 \\
\hline 4.27 & $14.00)$ & 375.00 \\
\hline 6.10 & $20.00)$ & 170.00 \\
\hline 9.14 & $30.00)$ & 75.00 \\
\hline 12.19 & $40.00)$ & 67.00 \\
\hline 18.29 & $60.00)$ & 67.00 \\
\hline 24.38 & $80.00)$ & 72.00 \\
\hline 30.48 & $100.00)$ & 77.00 \\
\hline 30.48 & $100.00)$ & 72.00 \\
\hline 42.67 & $140.00)$ & 77.00 \\
\hline
\end{tabular}

\begin{tabular}{|c|c|c|}
\hline 60.96 & $200.00)$ & 67.00 \\
\hline 91.44 & $300.00)$ & 60.00 \\
\hline 121.92 & $400.00)$ & 51.00 \\
\hline 91.44 & $300.00)$ & 54.00 \\
\hline 121.92 & $400.00)$ & 51.00 \\
\hline 182.88 & $600.00)$ & 49.00 \\
\hline 243.84 & $800.00)$ & 46.50 \\
\hline 304.80 & $1000.00)$ & 45.00 \\
\hline 304.80 & $1000.00)$ & 46.00 \\
\hline 426.72 & $1400.00)$ & 46.00 \\
\hline 609.60 & $2000.00)$ & 49.00 \\
\hline 914.40 & $3000.00)$ & 54.00 \\
\hline 914.40 & $3000.00)$ & 54.00 \\
\hline 1219.20 & $4000.00)$ & 58.00 \\
\hline 1828.80 & $6000.00)$ & 65.00 \\
\hline 2438.40 & $8000.00)$ & 89.00 \\
\hline
\end{tabular}

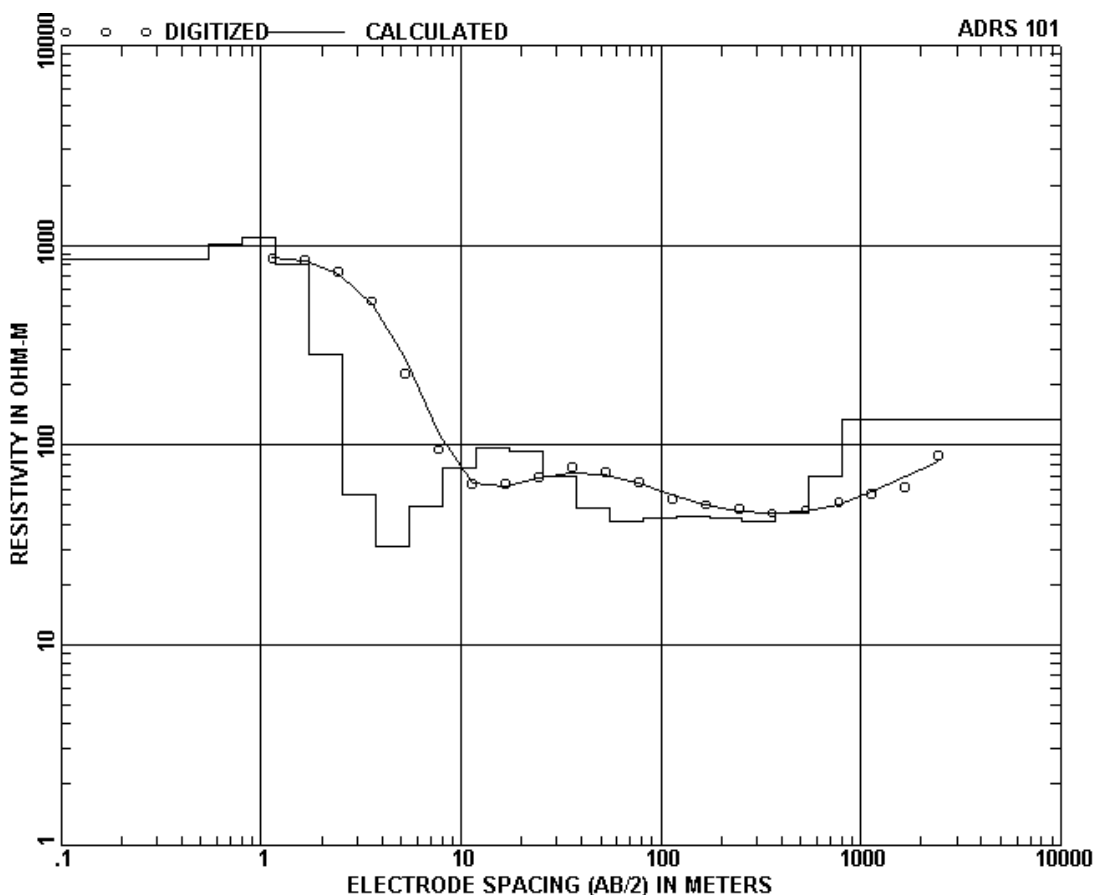

DEPTH, $m($ ft ) RESIS. DEPTH, $m($ ft ) RESIS.

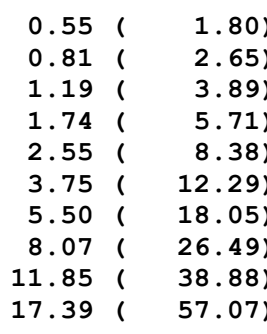

855.2
1003.5
1094.8
801.7
284.0
56.5
31.1
49.4
76.2
97.5

93.61

69.03

48.29

41.64

42.76

43.65

42.70

41.32

41.32

45.90

69.77 


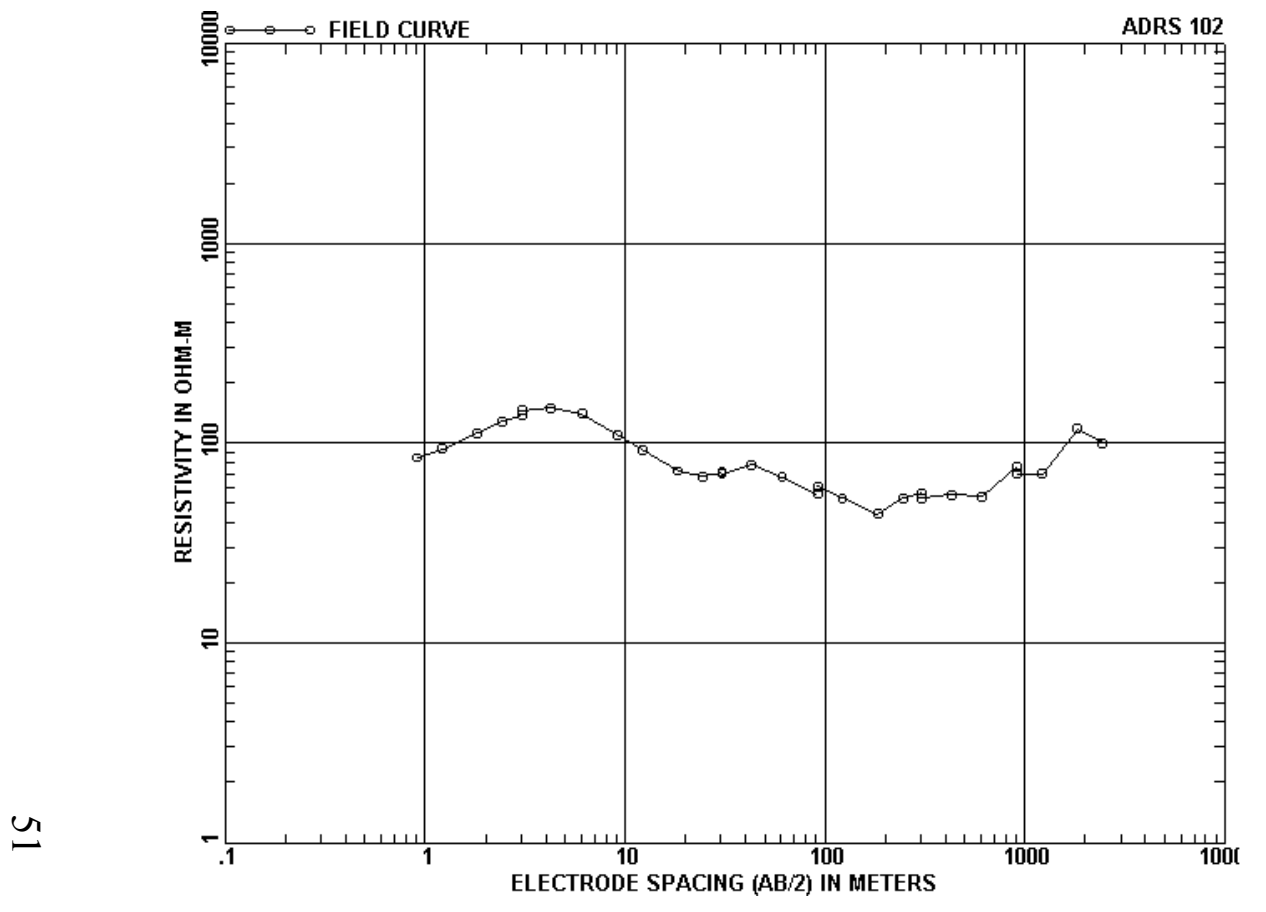

$A B / 2, m$ ( ft ) App. Res. $A B / 2$, m ( ft ) App. Res.

\begin{tabular}{|c|c|c|}
\hline 0.91 & $3.00)$ & 84.50 \\
\hline 1.22 & $4.00)$ & 93.20 \\
\hline 1.83 & $6.00)$ & 112.00 \\
\hline 2.44 & $8.00)$ & 128.00 \\
\hline 3.05 & $10.00)$ & 137.00 \\
\hline 3.05 & $10.00)$ & 146.00 \\
\hline 4.27 & $14.00)$ & 150.00 \\
\hline 6.10 & $20.00)$ & 140.00 \\
\hline 9.14 & $30.00)$ & 110.00 \\
\hline 12.19 & $40.00)$ & 92.10 \\
\hline 18.29 & $60.00)$ & 72.30 \\
\hline 24.38 & $80.00)$ & 67.80 \\
\hline 30.48 & $100.00)$ & 71.30 \\
\hline 30.48 & $100.00)$ & 69.90 \\
\hline 42.67 & $140.00)$ & 77.80 \\
\hline
\end{tabular}

\begin{tabular}{|c|c|c|}
\hline 60.96 & $200.00)$ & 67.30 \\
\hline 91.44 & $300.00)$ & 55.60 \\
\hline 91.44 & $300.00)$ & 60.40 \\
\hline 121.92 & $400.00)$ & 52.90 \\
\hline 182.88 & $600.00)$ & 44.20 \\
\hline 243.84 & $800.00)$ & 53.00 \\
\hline 304.80 & $(1000.00)$ & 56.00 \\
\hline 304.80 & $(1000.00)$ & 53.00 \\
\hline 426.72 & $(1400.00)$ & 55.00 \\
\hline 609.60 & $(2000.00)$ & 54.00 \\
\hline 914.40 & $3000.00)$ & 76.00 \\
\hline 914.40 & $3000.00)$ & 70.00 \\
\hline 1219.20 & $(4000.00)$ & 70.00 \\
\hline 1828.80 & $6000.00)$ & 118.00 \\
\hline 438.40 & $8000.00)$ & 00 \\
\hline
\end{tabular}

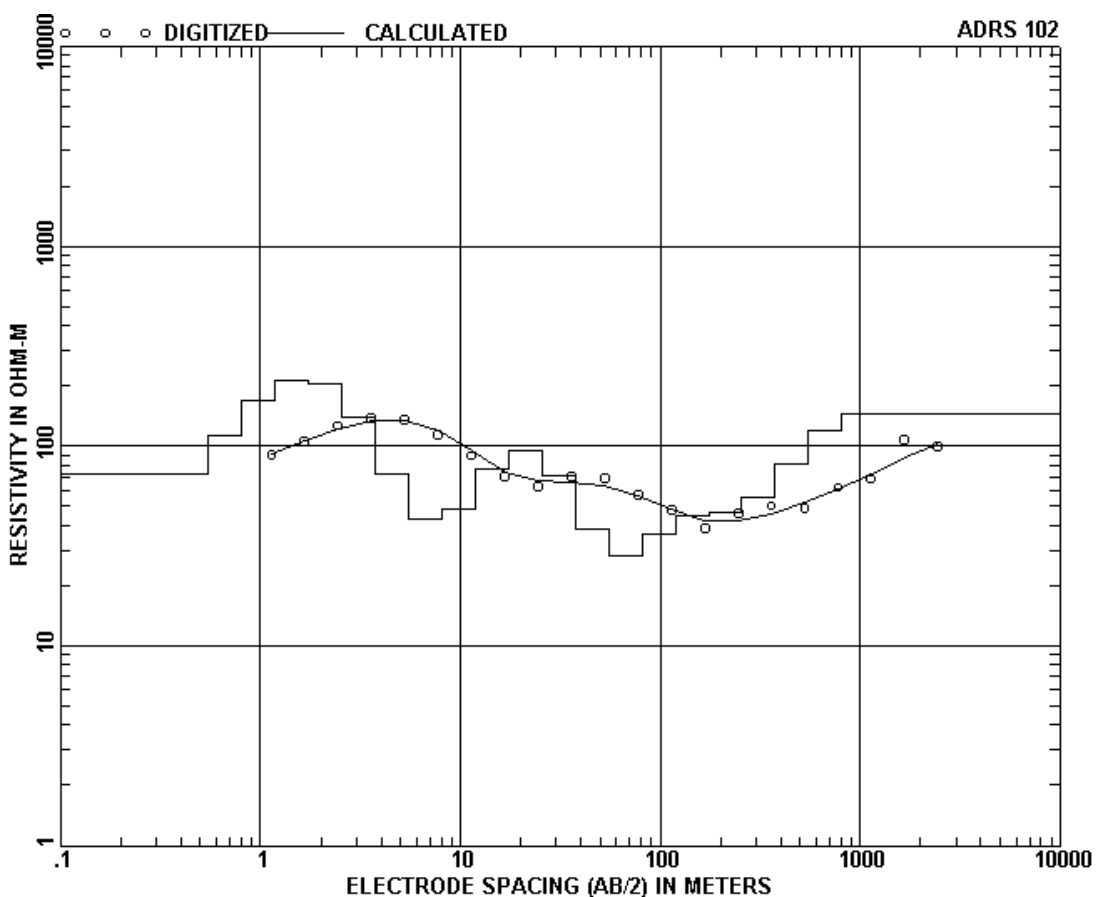

DEPTH, $m$ ( ft ) RESIS. DEPTH, $m$ ( ft ) RESIS

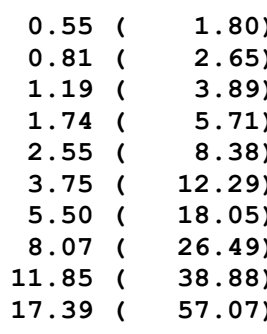

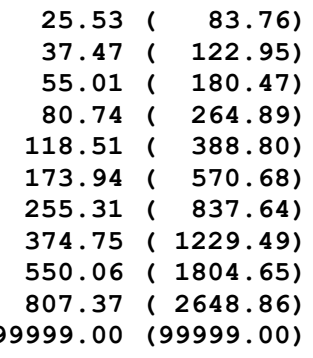

93.98

70.64

38.43

28.09

36.33

44.91

46.41

55.04

55.04

81.27
120.39

120.39 


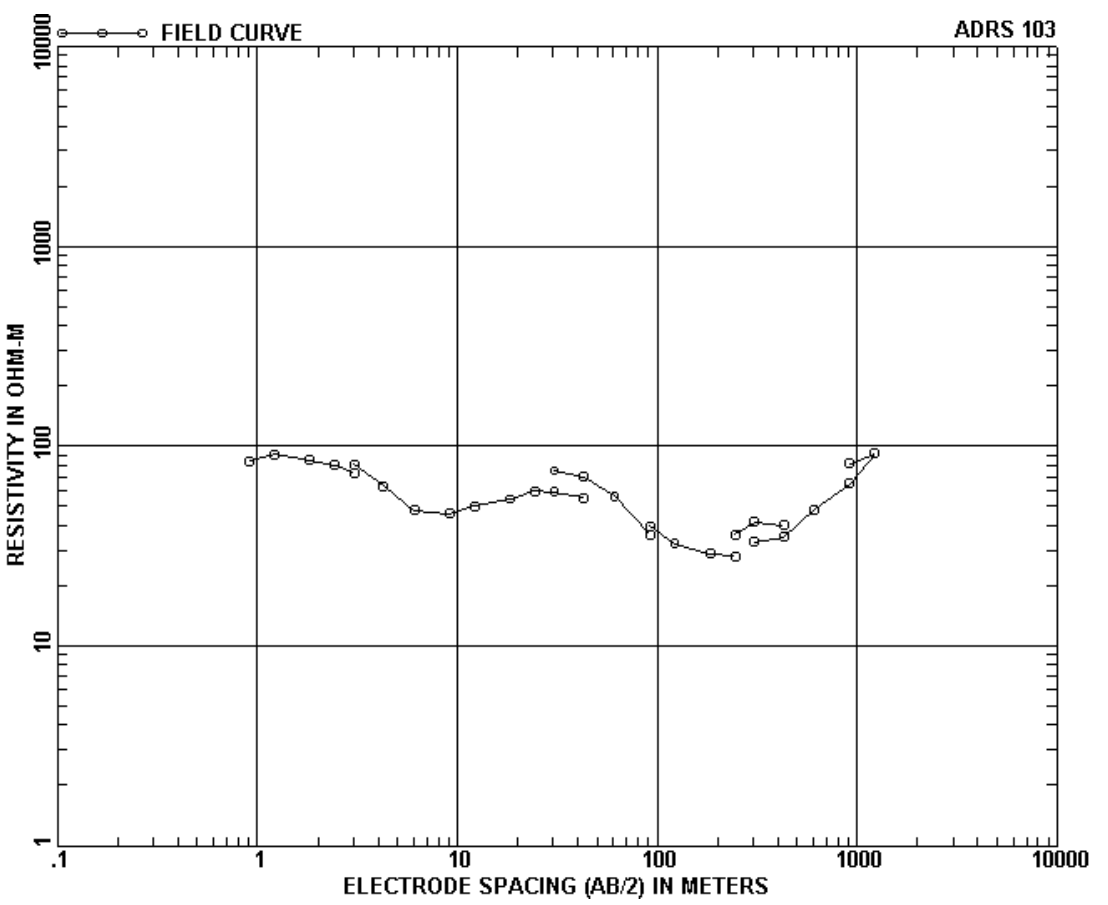

$A B / 2$, m ( ft ) App. Res. $A B / 2$, m ( ft ) App. Res.

\begin{abstract}
84.00
91.00

85.20

80.00

73.10

81.00

62.80

4.80

47.60

46.00

50.00

54.30

59.40

58.80

54.90

75.20

70.20
\end{abstract}

\begin{tabular}{|c|c|c|}
\hline 60.96 & $200.00)$ & 56.00 \\
\hline 91.44 & $300.00)$ & 36.00 \\
\hline 91.44 & $300.00)$ & 39.50 \\
\hline 121.92 & $400.00)$ & 32.40 \\
\hline 182.88 & $600.00)$ & 28.80 \\
\hline 243.84 & $800.00)$ & 28.10 \\
\hline 243.84 & $800.00)$ & 36.00 \\
\hline 304.80 & $(1000.00)$ & 41.80 \\
\hline 426.72 & $1400.00)$ & 40.00 \\
\hline 304.80 & $(1000.00)$ & 33.00 \\
\hline 426.72 & $1400.00)$ & 35.00 \\
\hline 609.60 & $2000.00)$ & 48.00 \\
\hline 914.40 & $3000.00)$ & 65.00 \\
\hline 1219.20 & $4000.00)$ & 92.00 \\
\hline 914.40 & $3000.00)$ & 82.00 \\
\hline & & \\
\hline
\end{tabular}

$1219.20(4000.00)$

92.00

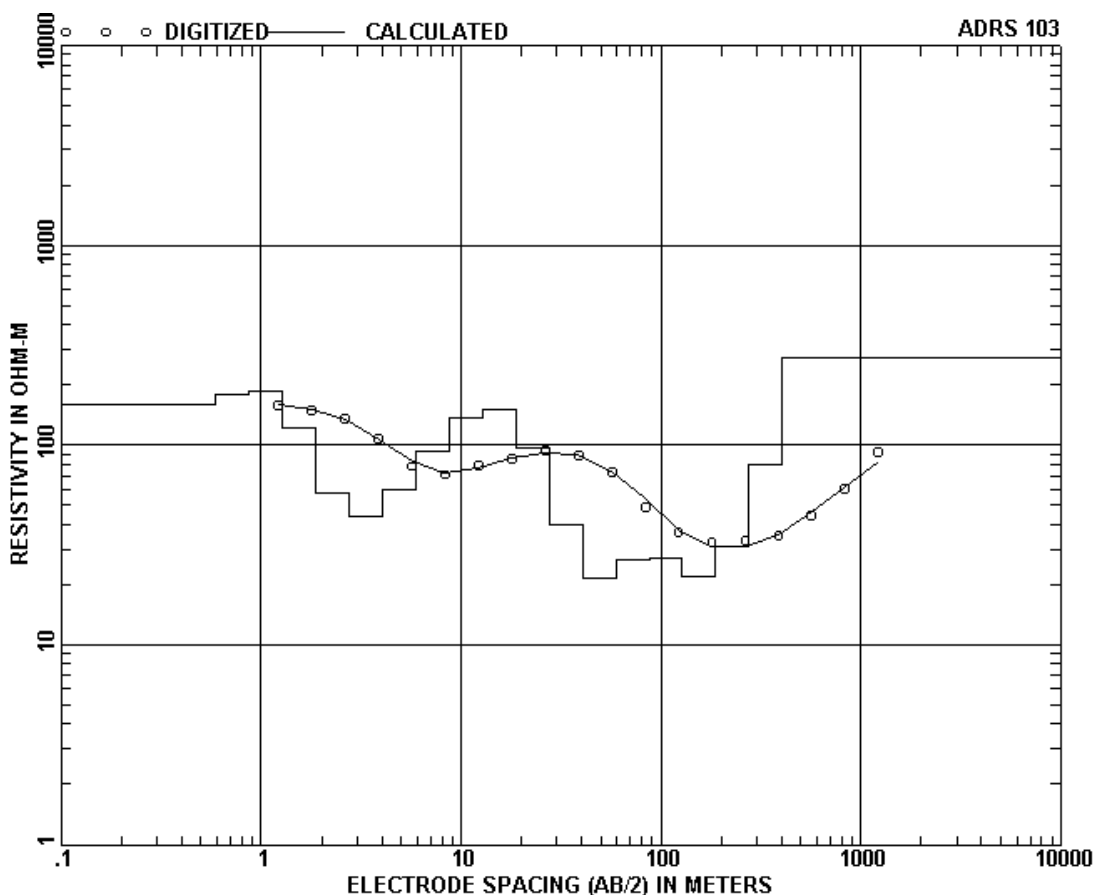

DEPTH, $m$ ( ft ) RESIS. DEPTH, $m($ ft ) RESIS.

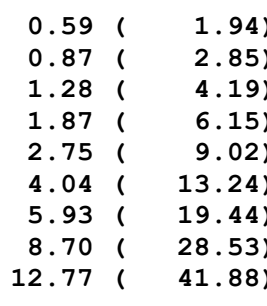

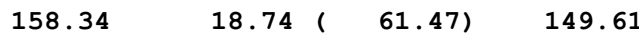

180.04

$18.74(61.47)$

149.61

$27.50(90.23) \quad 96.77$

$40.37(132.44) \quad 39.55$

$59.25(194.40) \quad 21.47$

$86.97(285.34) \quad 26.71$

$127.66(418.82) \quad 27.32$

$275.03(902.33) \quad 31.02$

$403.69(1324.43)$

$99999.00(99999.00) \quad 275.09$
$187.37(614.75)$ 


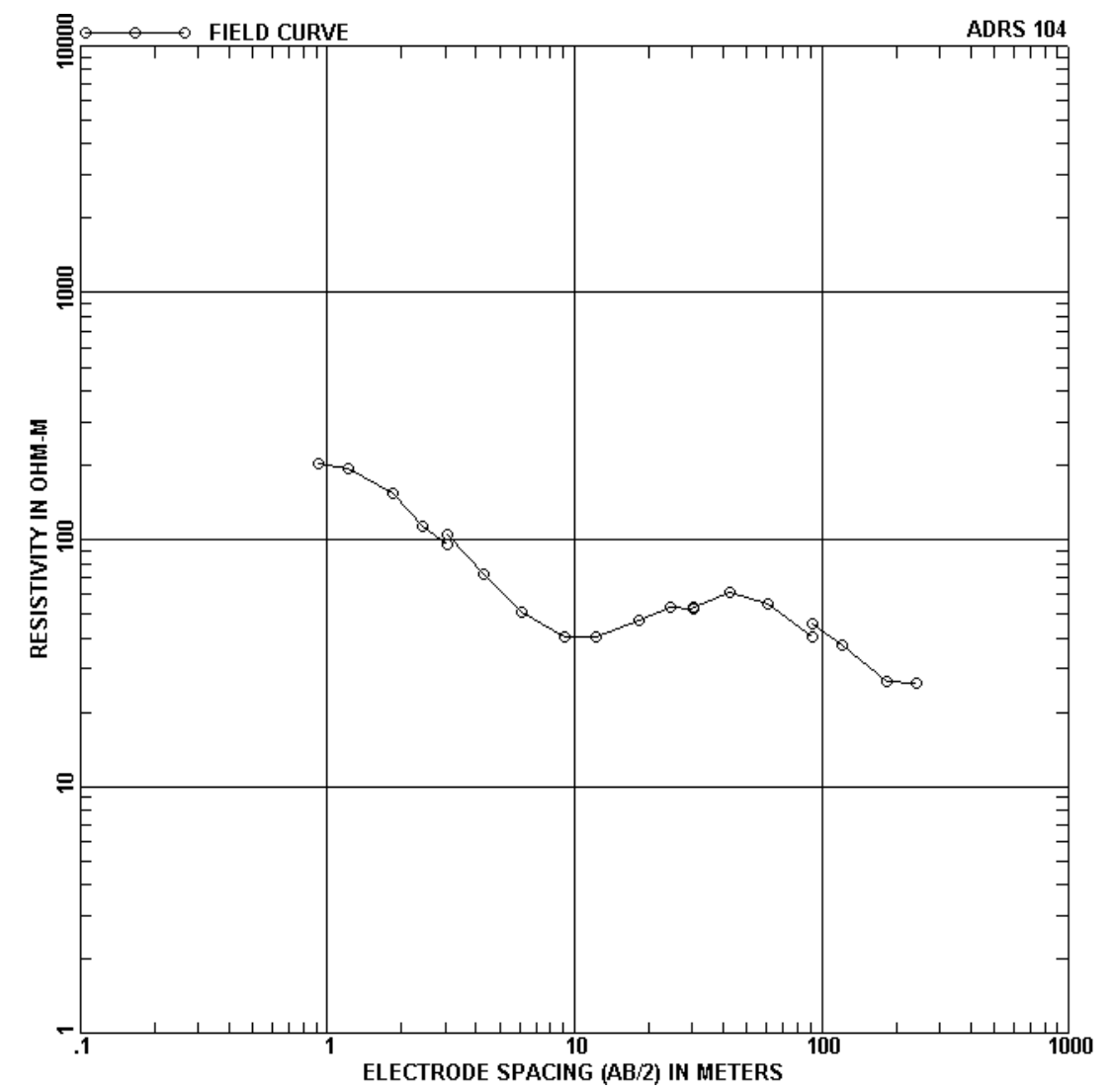

$A B / 2, m(f t)$ App. Res. $A B / 2, m$ ( ft ) App. Res.

\begin{tabular}{|c|c|c|}
\hline 0.91 & $3.00)$ & 204.00 \\
\hline 1.22 & $4.00)$ & 193.00 \\
\hline 1.83 & $6.00)$ & 154.00 \\
\hline 2.44 & $8.00)$ & 113.00 \\
\hline 3.05 & $10.00)$ & 95.40 \\
\hline 3.05 & $10.00)$ & 104.00 \\
\hline 4.27 & $14.00)$ & 71.90 \\
\hline 6.10 & $20.00)$ & 50.80 \\
\hline 9.14 & $30.00)$ & 40.10 \\
\hline 12.19 & $40.00)$ & 40.20 \\
\hline
\end{tabular}

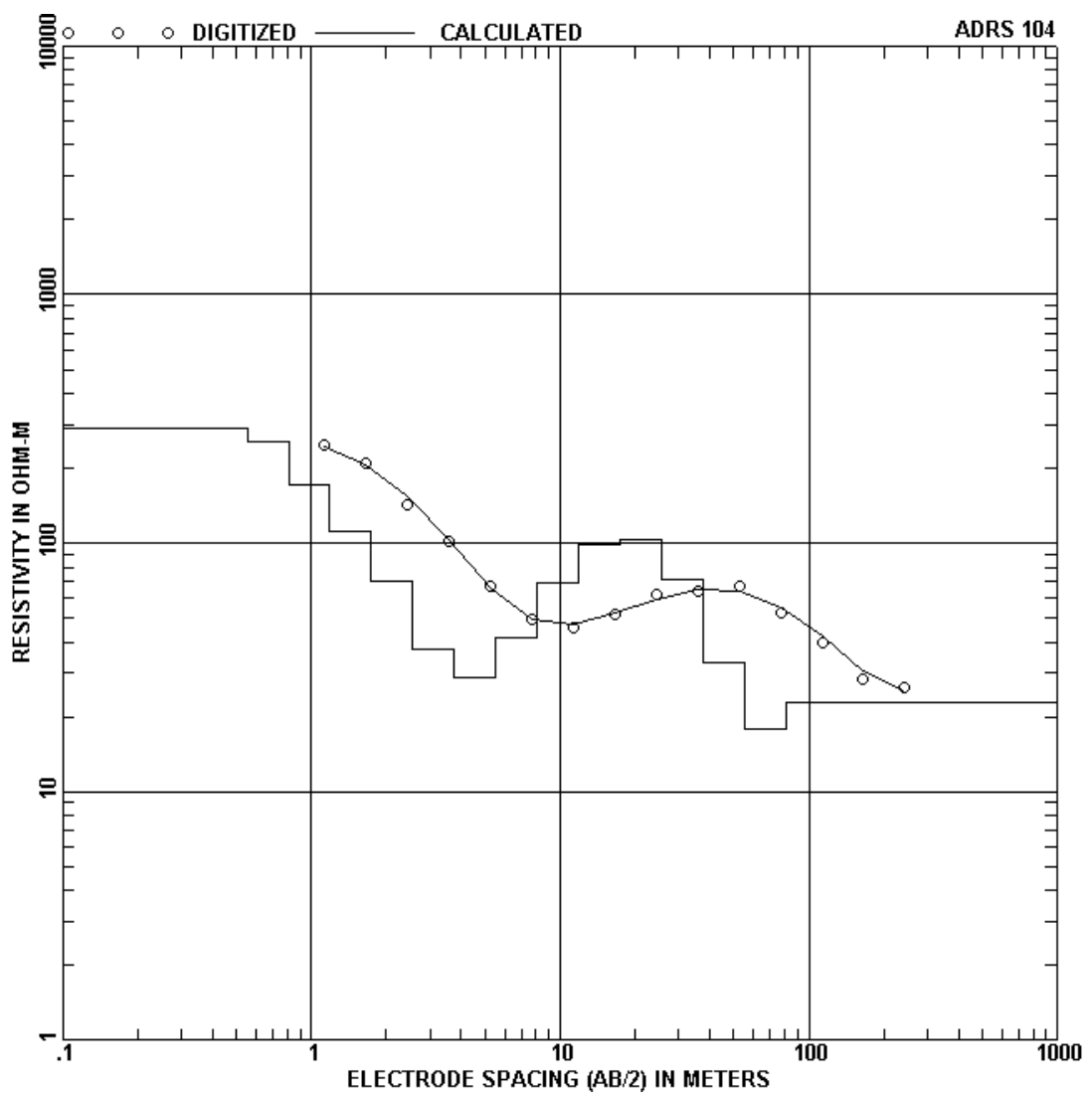

DEPTH, $m$ ( ft ) RESIS. DEPTH, $m($ ft ) RESIS. .
288.29

256.70

172.34

110.93

70.69

37.33

28.66

$8.07(\quad 26.49)$
$11.85(\quad 38.88)$ $\begin{array}{ll}11.85 & (38.88) \\ 17.39 & (\quad 57.07)\end{array}$

$17.39(57.07)$
$25.53(83.76)$ $37.47(122.95)$ $55.01(180.47)$

$80.74(264.89)$ $99999.00 \quad(99999.00)$
41.45

68.67

98.05

103.56

71.13

33.12

17.94 

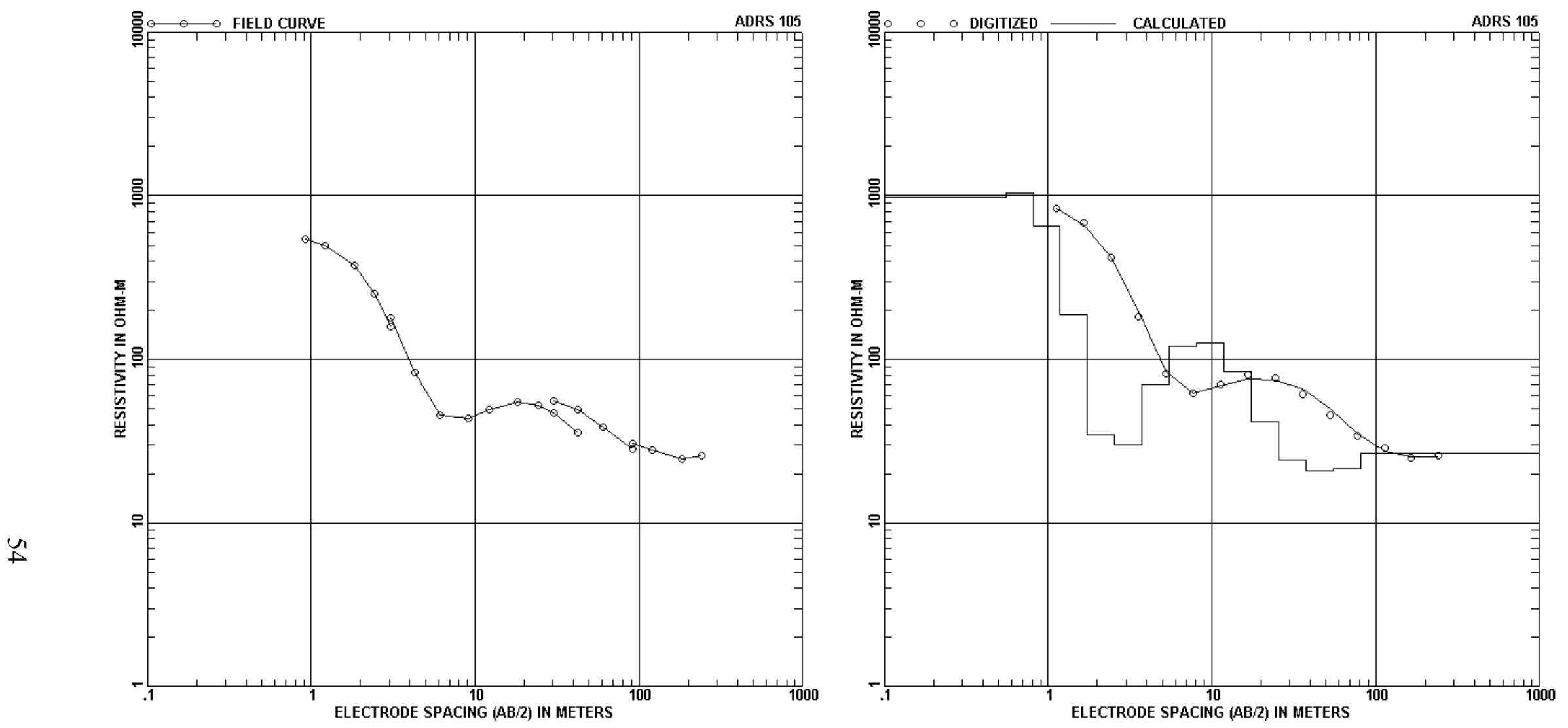

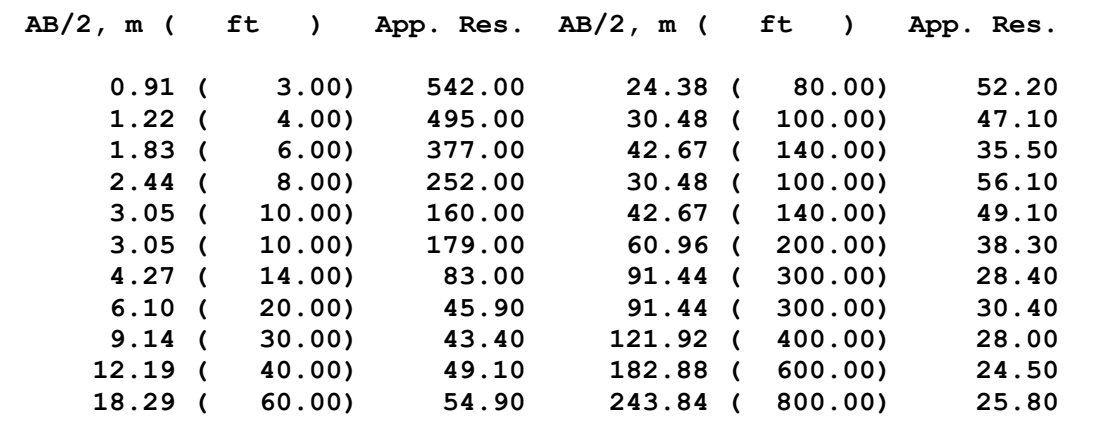

\begin{tabular}{|c|c|c|c|c|c|}
\hline DEPTH， m ( & ft & RESIS. & DEPTH， m & ft & RESIS. \\
\hline 0.551 & $1.80)$ & 983.55 & 8.07 & $26.49)$ & 120.02 \\
\hline $0.81 i$ & $2.65)$ & 1033.81 & 11.85 & $38.88)$ & 126.94 \\
\hline 1.19 ( & $3.89)$ & 652.68 & 17.39 & $57.07)$ & 83.92 \\
\hline $1.74 i$ & $5.71)$ & 187.22 & 25.53 & $83.76)$ & 41.83 \\
\hline 2.551 & $8.38)$ & 34.36 & 37.47 & $122.95)$ & 24.30 \\
\hline $3.75 i$ & $12.29)$ & 30.05 & 55.01 & ( 180.47$)$ & 20.66 \\
\hline $5.50 i$ & $18.05)$ & 69.86 & 80.74 & $264.89)$ & 21.43 \\
\hline & & & 99999.00 & $(99999.00)$ & 26.51 \\
\hline
\end{tabular}




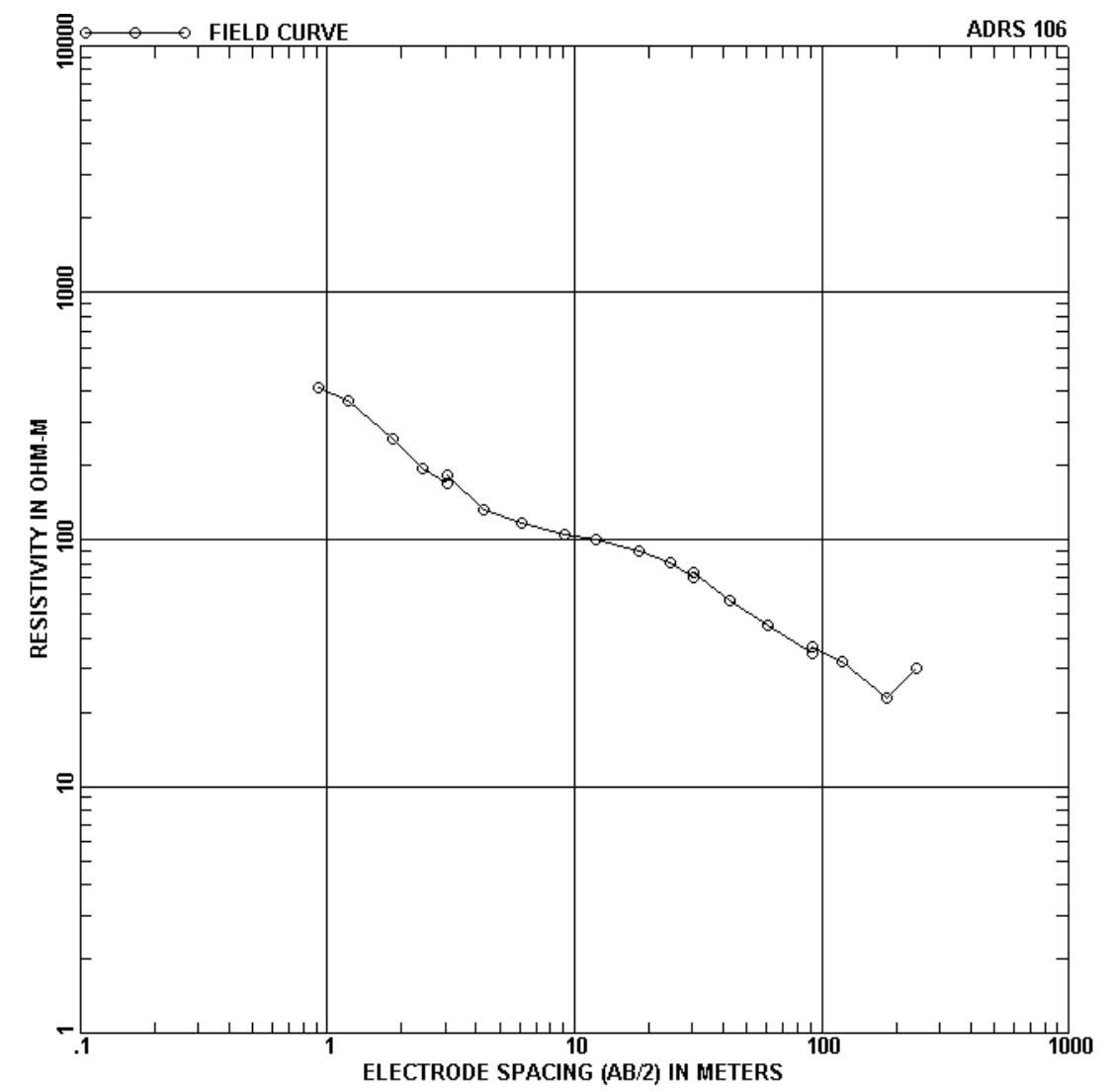

$A B / 2$, m ( ft ) App. Res. $A B / 2$, m ( ft ) App. Res.

\begin{tabular}{|c|c|c|}
\hline 0.91 & $3.00)$ & 415.00 \\
\hline 1.22 & $4.00)$ & 362.00 \\
\hline 1.83 & $6.00)$ & 254.00 \\
\hline 2.44 & $8.00)$ & 194.00 \\
\hline 3.05 & $10.00)$ & 168.00 \\
\hline 3.05 & $10.00)$ & 181.00 \\
\hline 4.27 & $14.00)$ & 131.00 \\
\hline 6.10 & $20.00)$ & 116.00 \\
\hline 9.14 & $30.00)$ & 105.00 \\
\hline 12.19 & $40.00)$ & 99.30 \\
\hline
\end{tabular}
$30.48(100.00)$

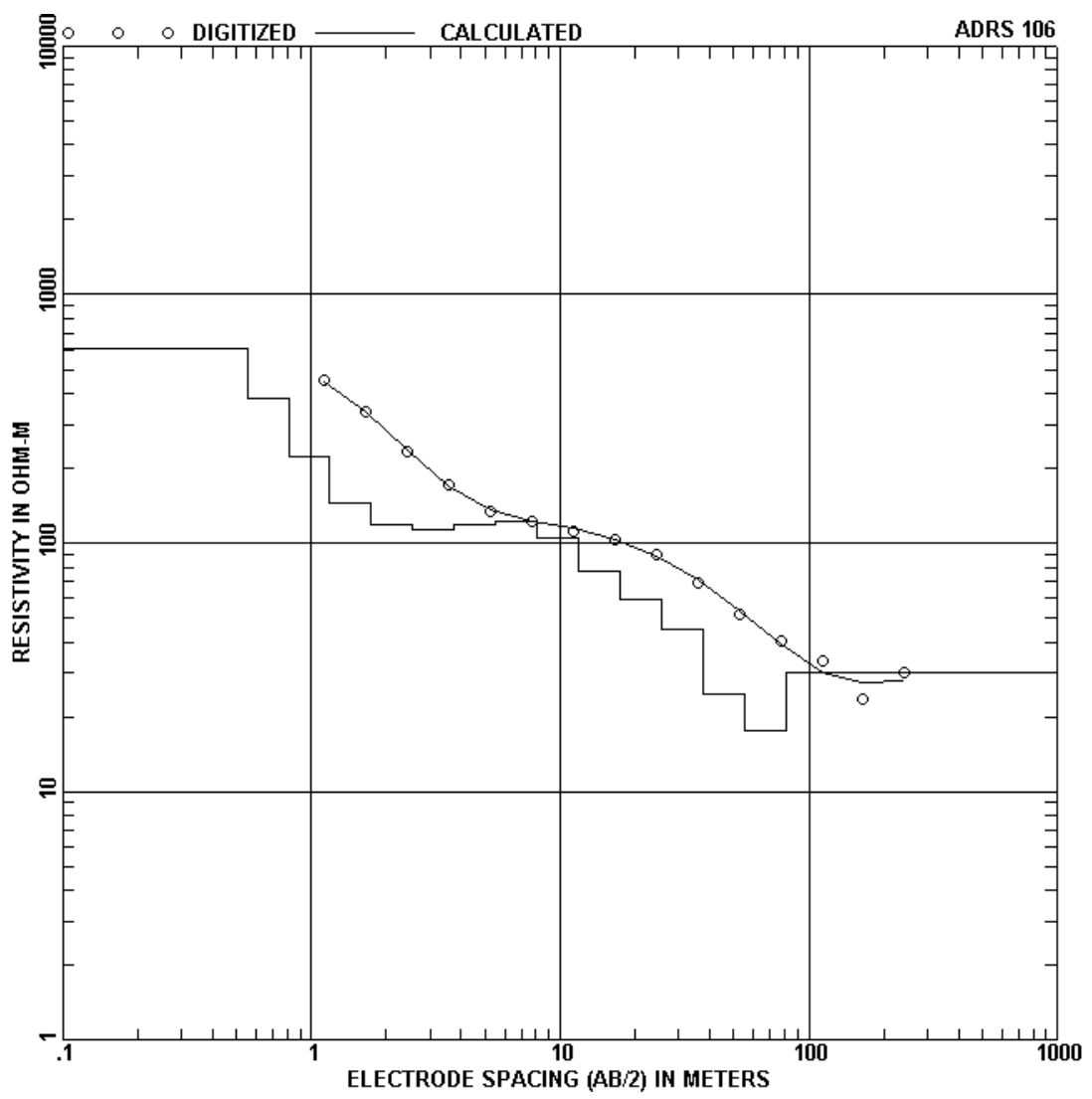

DEPTH, $m$ ( ft ) RESIS. DEPTH, $m$ ( ft ) RESIS.
105.14

77.51

59.30

45.07

24.81

17.49 30.29

90.10
81.10
70.50
74.10
56.30
44.60
34.50
36.60
31.90
22.80
30.00

122.50 $18.29(60.00)$ $30.48(100.00)$ $42.67(140.00)$
$60.96(200.00)$ $91.44(300.00)$ $91.44(300.00)$ $121.92(400.00)$ 82.88 ( 600.00$)$ $243.84(800.00)$

\begin{tabular}{|c|c|c|}
\hline 0.55 & $1.80)$ & 605.34 \\
\hline 0.81 & $2.65)$ & 381.86 \\
\hline 1.19 & $3.89)$ & 222.86 \\
\hline 1.74 & $5.71)$ & 145.32 \\
\hline 2.55 & $8.38)$ & 117.84 \\
\hline 3.75 & $12.29)$ & 113.23 \\
\hline 5.50 & $18.05)$ & 119.23 \\
\hline
\end{tabular}

$8.07(26.49)$ 11.85 ( 38.88$)$ 17.39 ( 57.07) 25.53 ( 83.76) 37.47 ( 122.95) 55.01 ( 180.47) $80.74(264.89)$ $99999.00 \quad(99999.00)$ 


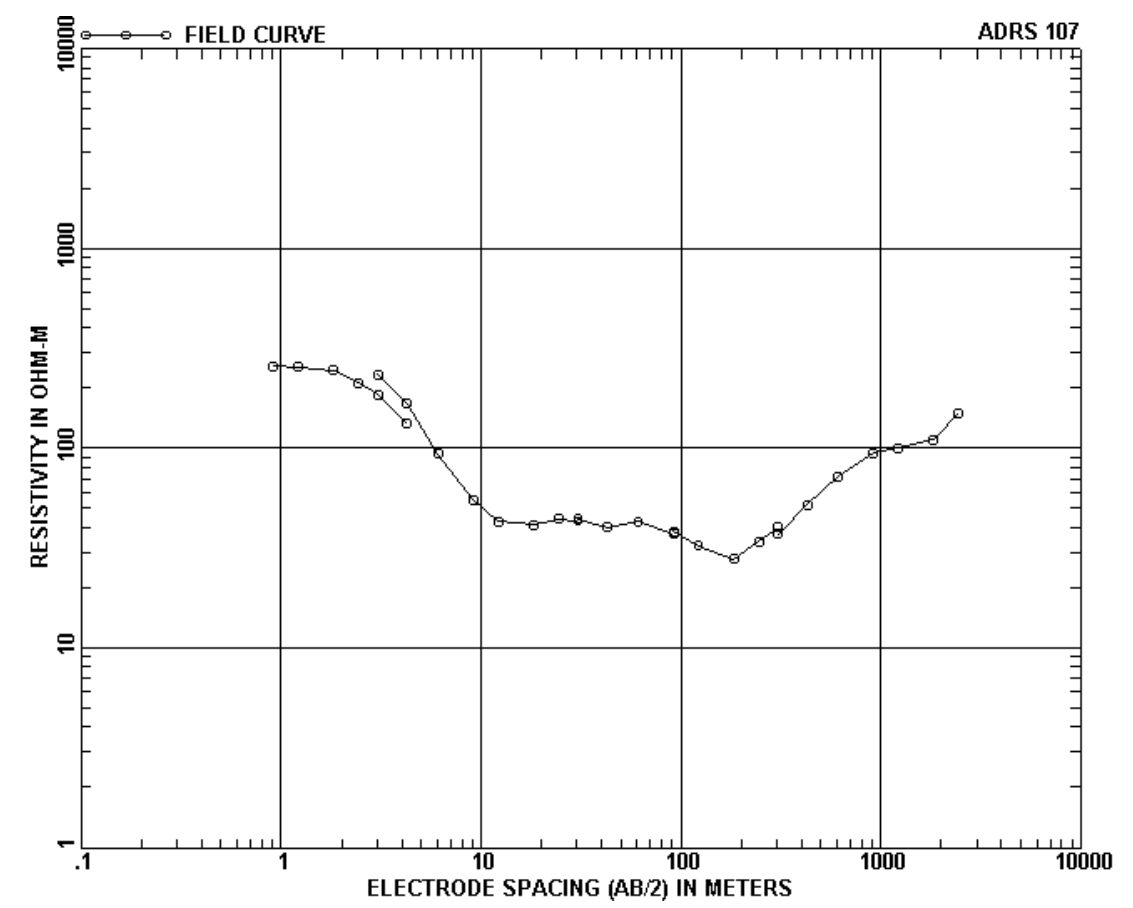

$A B / 2$, m (ft ) App. Res. $A B / 2$, m ( ft ) App. Res.

\begin{tabular}{|c|c|c|}
\hline 0.91 & $3.00)$ & 256.00 \\
\hline 1.22 & $4.00)$ & 255.00 \\
\hline 1.83 & $6.00)$ & 245.00 \\
\hline 2.44 & $8.00)$ & 211.00 \\
\hline 3.05 & $10.00)$ & 185.00 \\
\hline 4.27 & $14.00)$ & 132.00 \\
\hline 3.05 & $10.00)$ & 233.00 \\
\hline 4.27 & $14.00)$ & 167.00 \\
\hline 6.10 & $20.00)$ & 93.20 \\
\hline 9.14 & $30.00)$ & 55.00 \\
\hline 12.19 & $40.00)$ & 42.90 \\
\hline 18.29 & $60.00)$ & 41.10 \\
\hline 24.38 & $80.00)$ & 44.10 \\
\hline 30.48 & $100.00)$ & 43.00 \\
\hline 30.48 & $100.00)$ & 44.20 \\
\hline
\end{tabular}

\begin{tabular}{|c|c|c|}
\hline 42.67 & $140.00)$ & 40.10 \\
\hline 60.96 & $200.00)$ & 42.70 \\
\hline 91.44 & $300.00)$ & 37.20 \\
\hline 91.44 & $300.00)$ & 38.20 \\
\hline 121.92 & $400.00)$ & 32.50 \\
\hline 182.88 & $600.00)$ & 27.90 \\
\hline 243.84 & $800.00)$ & 34.00 \\
\hline 304.80 & $(1000.00)$ & 40.00 \\
\hline 304.80 & $(1000.00)$ & 37.00 \\
\hline 426.72 & $(1400.00)$ & 52.00 \\
\hline 609.60 & $2000.00)$ & 72.00 \\
\hline 914.40 & $(3000.00)$ & 94.00 \\
\hline 914.40 & $(3000.00)$ & 94.00 \\
\hline 1219.20 & $4000.00)$ & 100.00 \\
\hline 1828.80 & $6000.00)$ & 110.00 \\
\hline & & \\
\hline
\end{tabular}

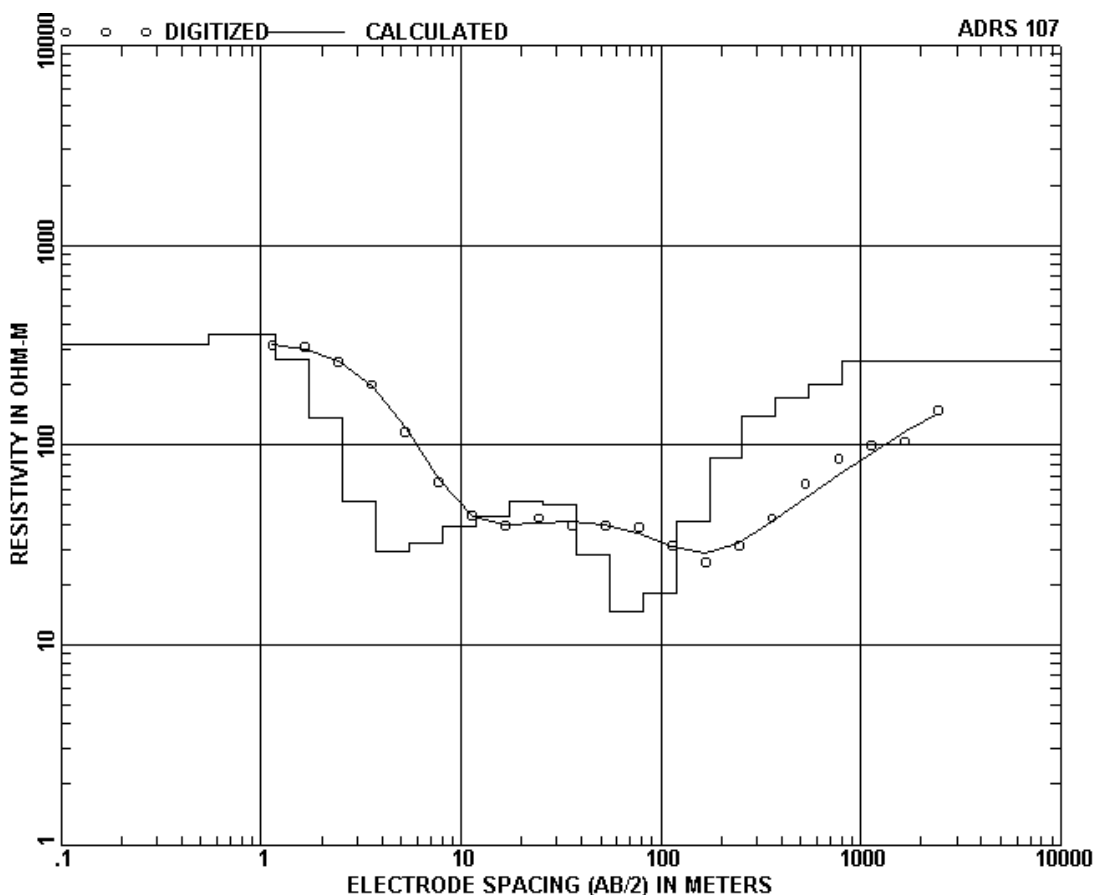

DEPTH, m ( ft ) RESIS. DEPTH, m ( ft ) RESIS.

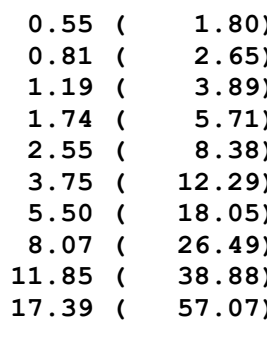

(1)

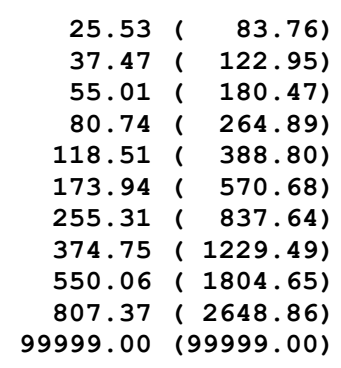

51.90 49.77 28.23 14.69 18.21 41.17 86.63 138.13 138.13 172.69 200.55 

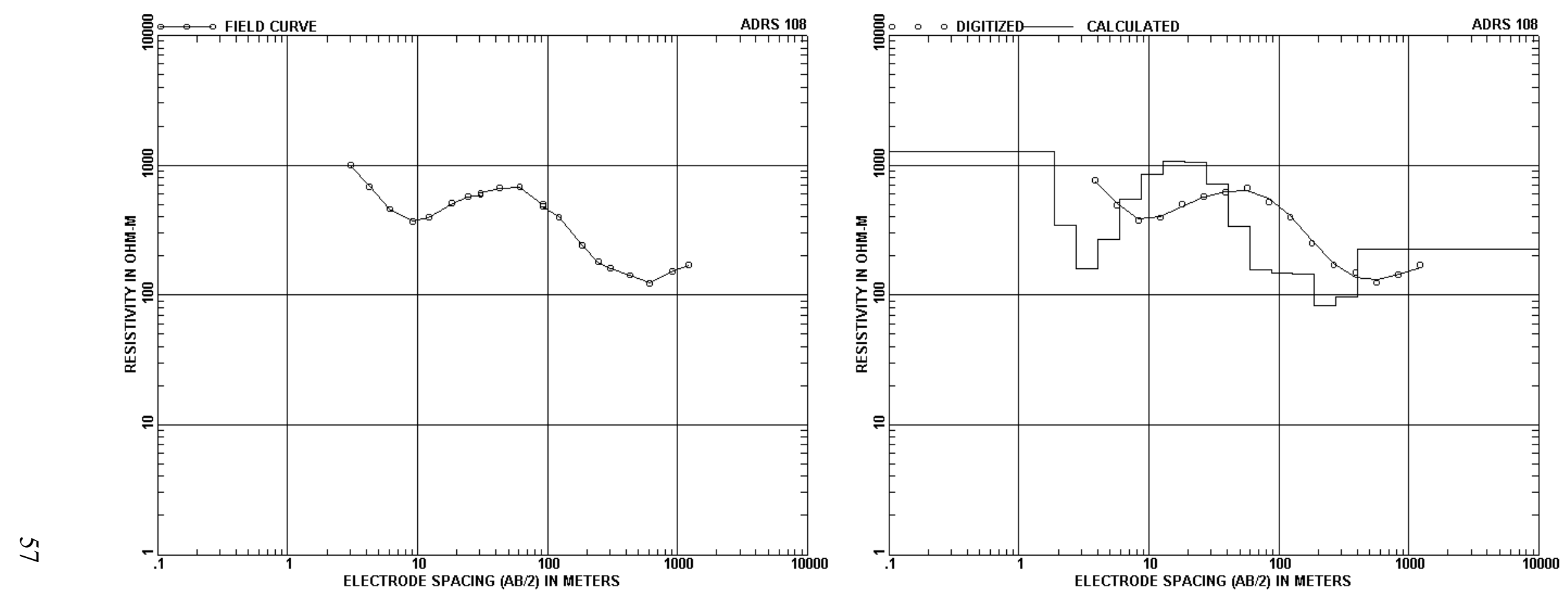

$A B / 2$, $m$ ( ft ) App. Res. $A B / 2$, $m$ ( ft ) App. Res.

DEPTH, $m$ ( ft ) RESIS. DEPTH, m ( ft ) RESIS.

\begin{tabular}{|c|c|c|c|c|c|}
\hline 3.05 & $10.00)$ & 1000.00 & 91.44 & $300.00)$ & 500.00 \\
\hline 4.27 & $14.00)$ & 683.00 & 91.44 & $300.00)$ & 480.00 \\
\hline 6.10 & $20.00)$ & 460.00 & 121.92 & $400.00)$ & 400.00 \\
\hline 9.14 & $30.00)$ & 370.00 & 182.88 & $600.00)$ & 242.00 \\
\hline 12.19 & $40.00)$ & 397.00 & 243.84 & $800.00)$ & 180.00 \\
\hline 18.29 & $60.00)$ & 510.00 & 304.80 & $(1000.00)$ & 160.00 \\
\hline 24.38 & $80.00)$ & 570.00 & 304.80 & $(1000.00)$ & 160.00 \\
\hline 30.48 & $100.00)$ & 590.00 & 426.72 & $1400.00)$ & 142.00 \\
\hline 30.48 & $100.00)$ & 610.00 & 609.60 & $2000.00)$ & 123.00 \\
\hline 42.67 & $140.00)$ & 665.00 & 914.40 & $3000.00)$ & 152.00 \\
\hline 60.96 & $200.00)$ & 680.00 & 1219.20 & $4000.00)$ & 170.00 \\
\hline
\end{tabular}

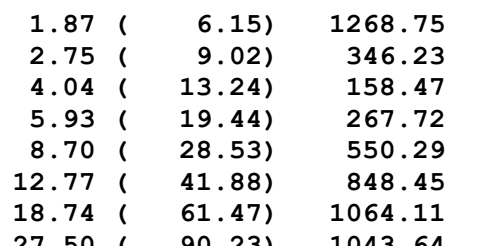

$\begin{array}{lll}40.37 & (132.44) & 714.81 \\ 59.25 & (194.40) & 337.71\end{array}$

$\begin{array}{lll}59.25 & (194.40) & 337.71 \\ 86.97 & (285.34) & 157.32\end{array}$

$127.66(418.82) \quad 146.76$

187.37 ( 614.75$) \quad 143.82$

$275.03(902.33) \quad 82.77$

$\begin{aligned} 403.69 & (1324.43) \\ & 97.39\end{aligned}$

$60.96(200.00) \quad 680.00 \quad 1219.20(4000.00) \quad 170.00$ 

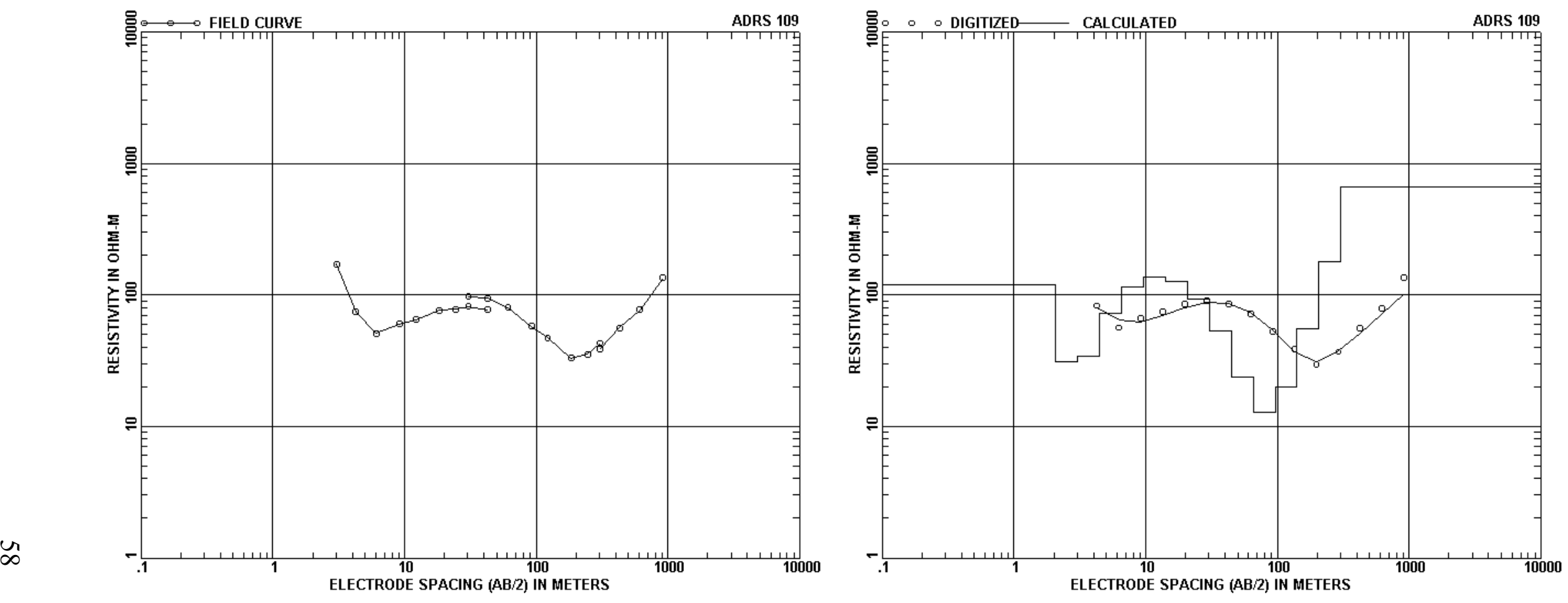

$A B / 2, m$ ( ft ) App. Res. $A B / 2$, m ( ft ) App. Res.

\begin{tabular}{|c|c|c|c|c|c|}
\hline 3.05 & $10.00)$ & 171.00 & 42.67 & $140.00)$ & 94.00 \\
\hline 4.27 & $14.00)$ & 74.00 & 60.96 & $200.00)$ & 80.00 \\
\hline 6.10 & $20.00)$ & 51.00 & 91.44 & $300.00)$ & 58.00 \\
\hline 9.14 & $30.00)$ & 60.00 & 121.92 & $400.00)$ & 47.00 \\
\hline 12.19 & $40.00)$ & 65.00 & 182.88 & $600.00)$ & 33.00 \\
\hline 18.29 & $60.00)$ & 76.00 & 243.84 & $800.00)$ & 35.20 \\
\hline 24.38 & $80.00)$ & 78.00 & 304.80 & $1000.00)$ & 43.00 \\
\hline 30.48 & $100.00)$ & 82.00 & 304.80 & $1000.00)$ & 39.00 \\
\hline 42.67 & $140.00)$ & 77.00 & 426.72 & $1400.00)$ & 56.00 \\
\hline \multirow[t]{2}{*}{30.48} & $100.00)$ & 97.00 & 609.60 & $2000.00)$ & 77.00 \\
\hline & & & 914.40 & $(3000.00)$ & 135.00 \\
\hline
\end{tabular}

DEPTH, $m$ ( ft ) RESIS. DEPTH, m ( ft )

RESIS.

\begin{tabular}{|c|c|c|c|c|c|}
\hline 2.06 & $6.77)$ & 119.59 & 30.28 & $99.33)$ & 92.17 \\
\hline 3.03 & $9.93)$ & 31.18 & 44.44 & $145.80)$ & 53.12 \\
\hline 4.44 & $14.58)$ & 34.35 & 65.23 & $214.01)$ & 23.64 \\
\hline 6.52 & $21.40)$ & 71.71 & 95.74 & $314.12)$ & 12.82 \\
\hline 9.57 & $31.41)$ & 114.42 & 140.53 & $461.06)$ & 19.98 \\
\hline 14.05 & $46.11)$ & 136.80 & 206.27 & $676.74)$ & 55.21 \\
\hline 20.63 & 67.67) & 126.84 & $\begin{array}{r}302.77 \\
\end{array}$ & $\begin{array}{r}993.32) \\
9999.00)\end{array}$ & $\begin{array}{l}177.33 \\
657.44\end{array}$ \\
\hline
\end{tabular}

56.00

$914.40(3000.00) \quad 135.00$ 

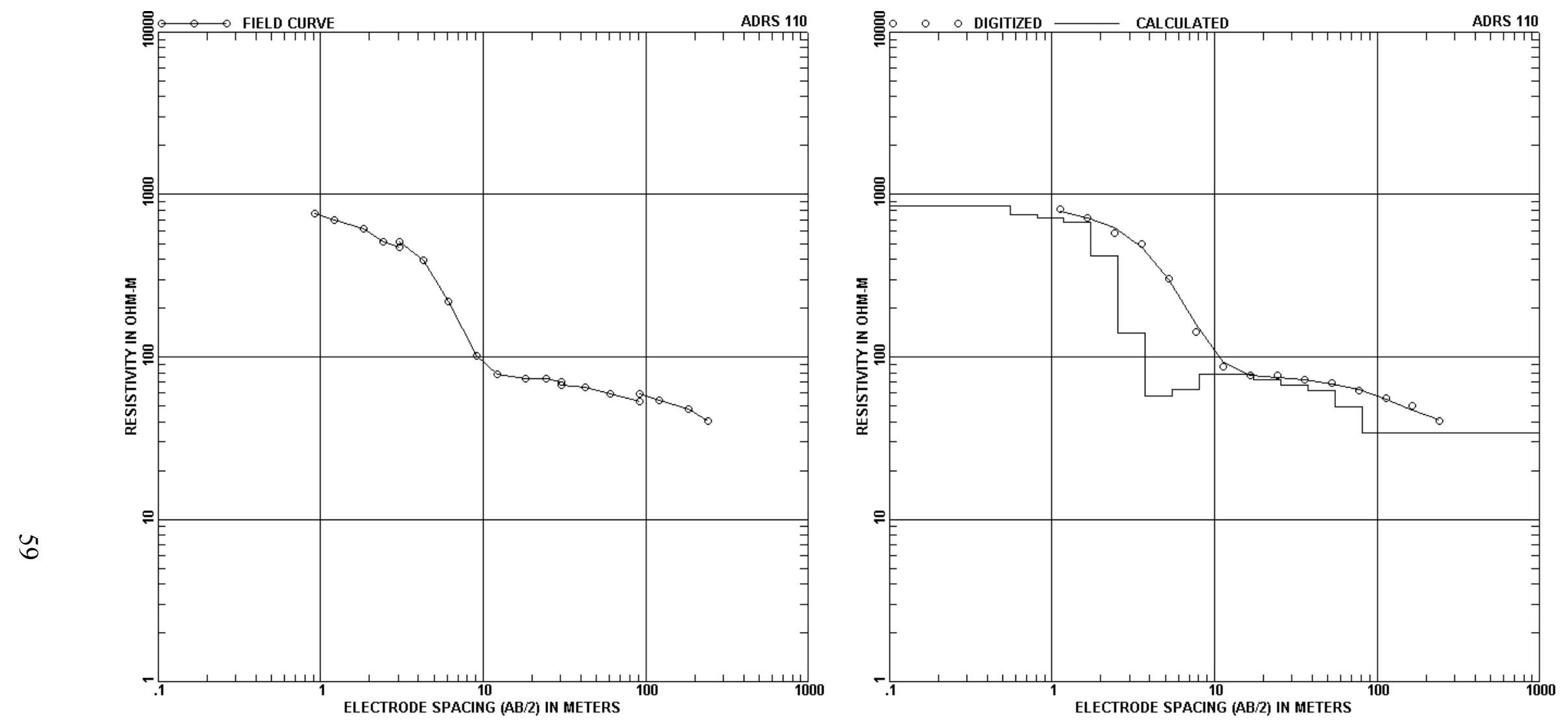

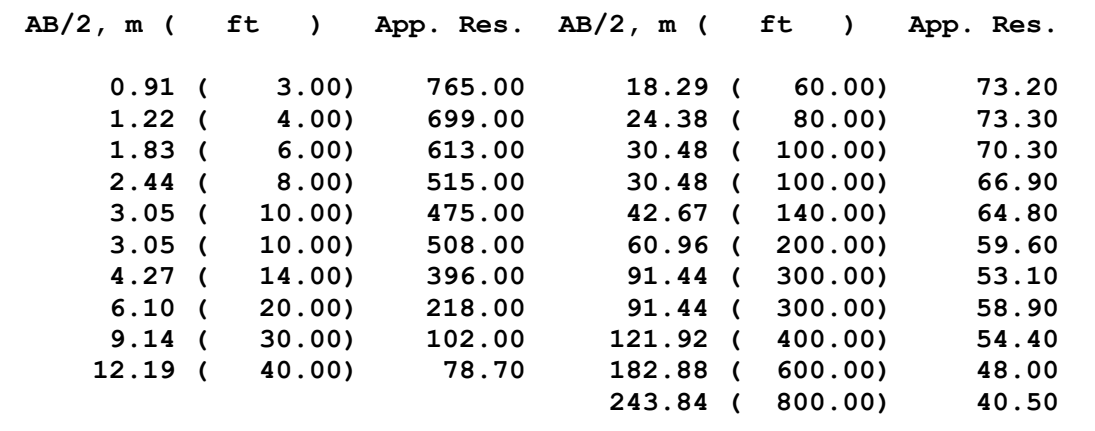

\begin{tabular}{|c|c|c|c|c|c|}
\hline DEPTH， m ( & ft, & RESIS. & DEPTH, m & ft & RESIS. \\
\hline 0.55 & $1.80)$ & 854.58 & 8.07 & $26.49)$ & 63.24 \\
\hline 0.81 & $2.65)$ & 752.73 & 11.85 & $38.88)$ & 77.96 \\
\hline 1.19 & $3.89)$ & 720.28 & 17.39 & $57.07)$ & 78.54 \\
\hline 1.74 & $5.71)$ & 675.46 & 25.53 & $83.76)$ & 72.04 \\
\hline 2.55 & $8.38)$ & 420.71 & 37.47 & $122.95)$ & 66.94 \\
\hline 3.75 & $12.29)$ & 140.34 & 55.01 & $180.47)$ & 61.83 \\
\hline 5.50 & $18.05)$ & 57.19 & 80.74 & ( 264.89$)$ & 49.60 \\
\hline & & & 99999.00 & $(99999.00)$ & 34.17 \\
\hline
\end{tabular}



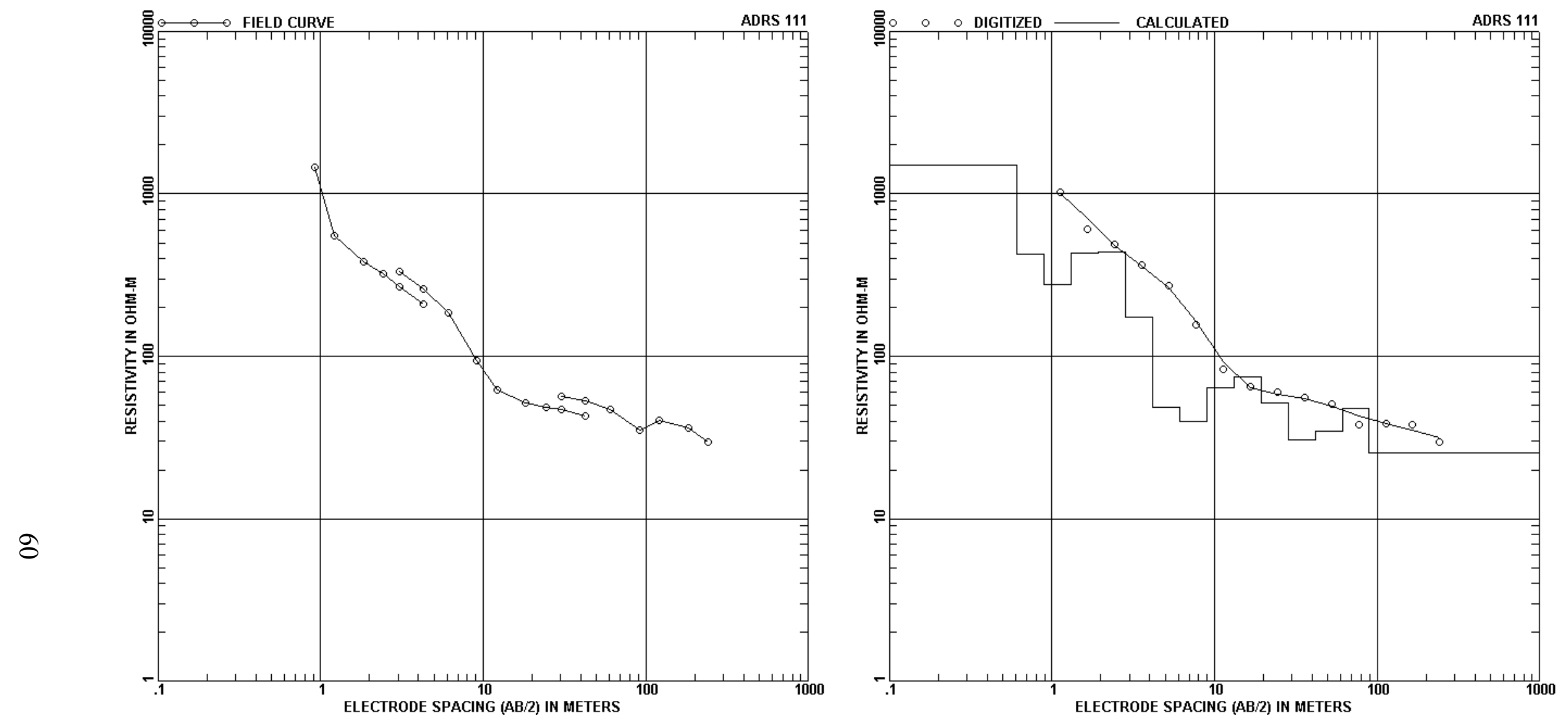

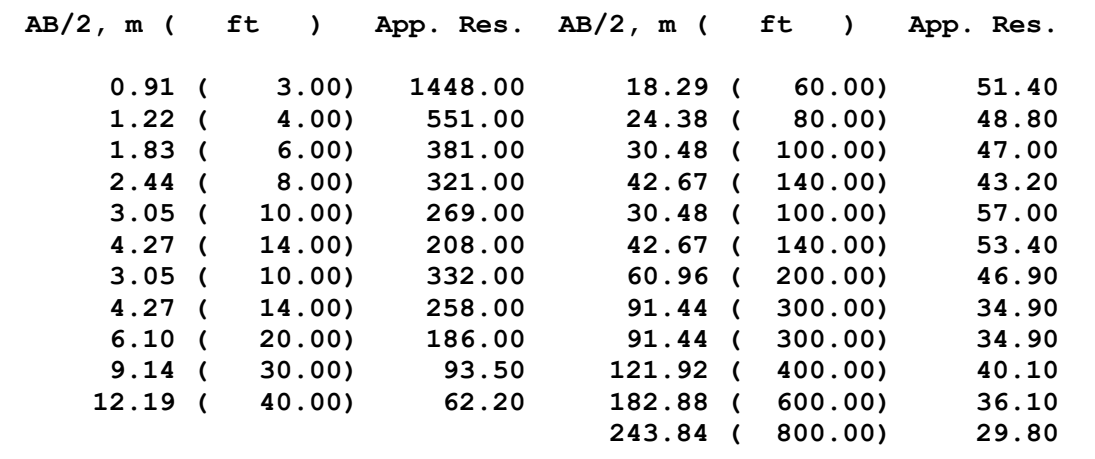

DEPTH， m ( ft )

$\begin{array}{rlr}0.61 & ( & 2.01) \\ 0.90 & ( & 2.94) \\ 1.32 & ( & 4.32) \\ 1.93 & ( & 6.34) \\ 2.84 & ( & 9.31) \\ 4.16 & ( & 13.66) \\ 6.11 & ( & 20.05)\end{array}$

)

DEPTH, m ( ft )

425.78

275.63

435.20

438.13

174.03

48.76

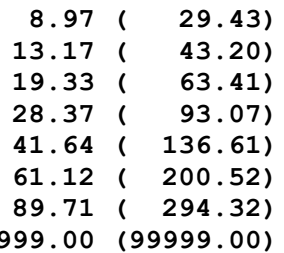

RESIS.

39.82

64.48

74.94

51.61

30.60

34.65

47.63

25.35 

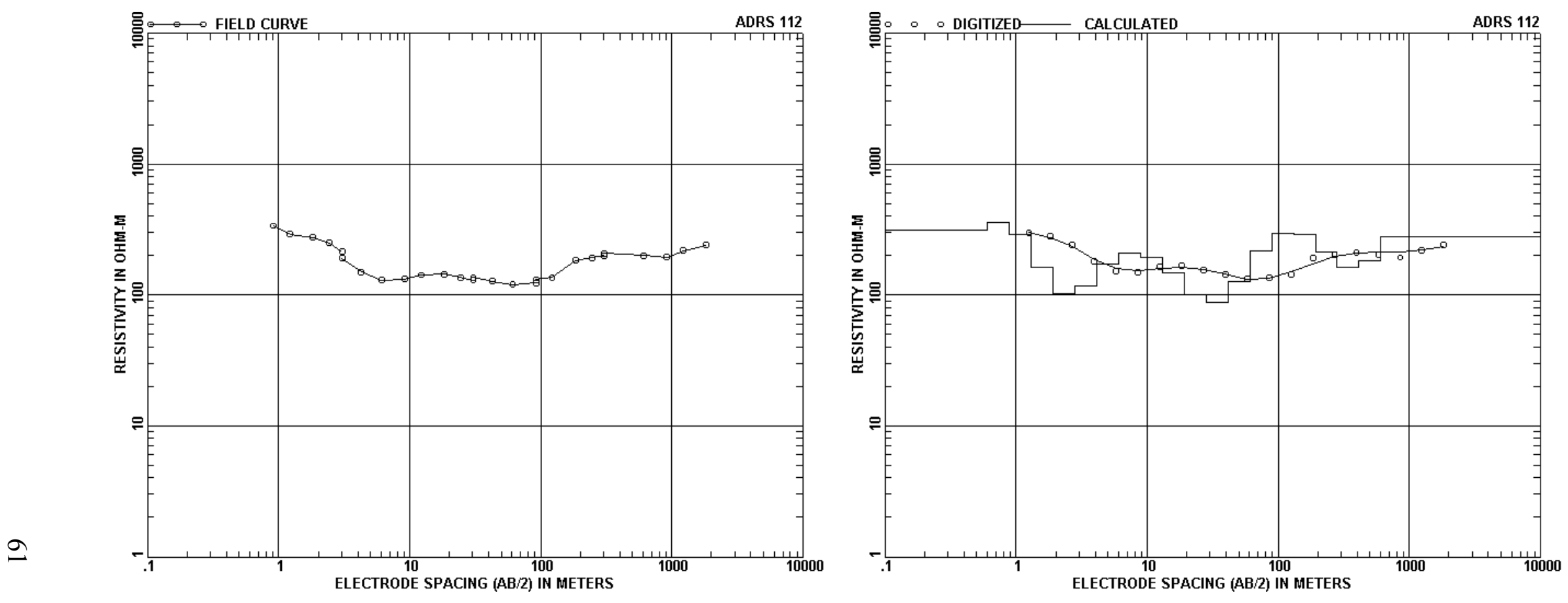

$A B / 2$, m (ft ) App. Res. $A B / 2$, m ( ft ) App. Res.

DEPTH, $m$ ( ft ) RESIS. DEPTH, m ( ft ) RESIS.

\begin{tabular}{|c|c|c|c|c|c|}
\hline 0.91 & $3.00)$ & 337.00 & 42.67 & $140.00)$ & 127. \\
\hline 1.22 & $4.00)$ & 291.00 & 60.96 & $200.00)$ & 120.00 \\
\hline 1.83 & $6.00)$ & 275.00 & 91.44 & $300.00)$ & 123.00 \\
\hline 2.44 & $8.00)$ & 249.00 & 91.44 & $300.00)$ & 131.0 \\
\hline 3.05 & $10.00)$ & 214.00 & 121.92 & $400.00)$ & $136 . C$ \\
\hline 3.05 & $10.00)$ & 190.00 & 182.88 & $600.00)$ & $184 . C$ \\
\hline 4.27 & $14.00)$ & 149.00 & 243.84 & $800.00)$ & 192.0 \\
\hline 6.10 & $20.00)$ & 130.00 & 304.80 & $1000.00)$ & $200 . C$ \\
\hline 9.14 & $30.00)$ & 132.00 & 304.80 & $1000.00)$ & 207.00 \\
\hline 12.19 & $40.00)$ & 142.00 & 609.60 & $2000.00)$ & $200 . C$ \\
\hline 18.29 & $60.00)$ & 144.00 & 914.40 & $3000.00)$ & $195 . C$ \\
\hline 24.38 & $80.00)$ & 136.00 & 914.40 & $3000.00)$ & 195.0 \\
\hline 30.48 & $100.00)$ & 130.00 & 1219.20 & $4000.00)$ & 218 . C \\
\hline 30.48 & $100.00)$ & 136.00 & 1828.80 & $6000.00)$ & 240.0 \\
\hline
\end{tabular}

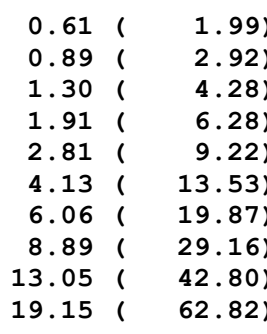

311.93

359.02

291.16

162.74

102.12

117.29

172.42

207.88

194.84

194.84

148.24

\begin{tabular}{|c|c|}
\hline 1 & \\
\hline$\ldots$ & 135 \\
\hline 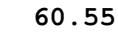 & 198. \\
\hline $8 . \varepsilon$ & 91. \\
\hline & \\
\hline 191. & 628. \\
\hline 281. & 922 . \\
\hline 412 . & 1353 \\
\hline & 1096 \\
\hline & \\
\hline
\end{tabular}

100.74

87.17

126.47

217.17

295.51

286.74

212.06

162.05

162.05

181.96 

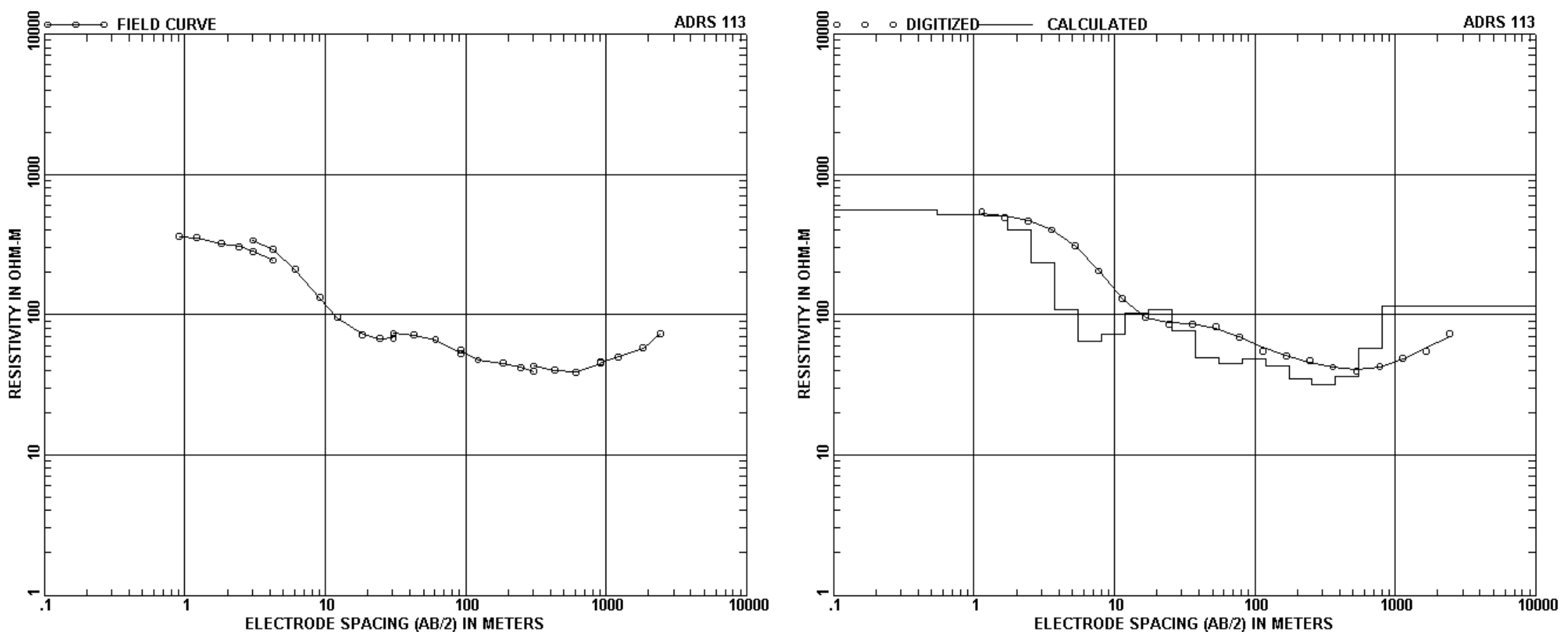

$A B / 2, \mathrm{~m}$ ( ft ) App. Res. $A B / 2, \mathrm{~m}$ ( ft ) App. Res.

DEPTH, $m$ ( ft ) RESIS. DEPTH, m ( ft ) RESIS.

\begin{tabular}{|c|c|c|c|c|c|}
\hline 0.91 & $3.00)$ & 361.00 & 42.67 & $(140.00)$ & 71.40 \\
\hline 1.22 & $4.00)$ & 352.00 & 60.96 & $200.00)$ & 66.10 \\
\hline 1.83 & $6.00)$ & 321.00 & 91.44 & $300.00)$ & 52.80 \\
\hline 2.44 & $8.00)$ & 306.00 & 91.44 & $300.00)$ & 55.70 \\
\hline 3.05 & $10.00)$ & 282.00 & 121.92 & $400.00)$ & 47.40 \\
\hline 4.27 & $14.00)$ & 243.00 & 182.88 & $600.00)$ & 45.00 \\
\hline 3.05 & $10.00)$ & 338.00 & 243.84 & $800.00)$ & 42.00 \\
\hline 4.27 & $14.00)$ & 290.00 & 304.80 & $(1000.00)$ & 39.30 \\
\hline 6.10 & $20.00)$ & 210.00 & 304.80 & $(1000.00)$ & 43.00 \\
\hline 9.14 & $30.00)$ & 132.00 & 426.72 & $(1400.00)$ & 40.00 \\
\hline 12.19 & $40.00)$ & 95.10 & 609.60 & $(2000.00)$ & 39.00 \\
\hline 18.29 & $60.00)$ & 72.20 & 914.40 & $(3000.00)$ & 45.00 \\
\hline 24.38 & $80.00)$ & 67.50 & 914.40 & $(3000.00)$ & 46.00 \\
\hline 30.48 & $100.00)$ & 68.10 & 1219.20 & $(4000.00)$ & 50.00 \\
\hline \multirow[t]{2}{*}{30.48} & $100.00)$ & 73.10 & 1828.80 & $(6000.00)$ & 58.00 \\
\hline & & & 2438.40 & $(8000.00)$ & 73.20 \\
\hline
\end{tabular}

\begin{tabular}{|c|c|}
\hline 0.55 & 1.80 \\
\hline 0.81 & 2.6 \\
\hline 1.19 & 3 \\
\hline 1.74 & \\
\hline 2.55 & 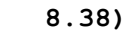 \\
\hline 3.75 & 12.2 \\
\hline 5.50 & 18. \\
\hline 8.07 & 26.4 \\
\hline 11.85 & 38.8 \\
\hline 17.39 & 57. \\
\hline
\end{tabular}

552.10

518.17

107.94

1.40

52.80

47.40

45.00

42.00

39.30

40.00

39.00
45.00

46.00

58.00

73.20

107.94

76.67

49.50

44.86

48.43

43.24

34.83

31.56

31.56

36.15

57.33 

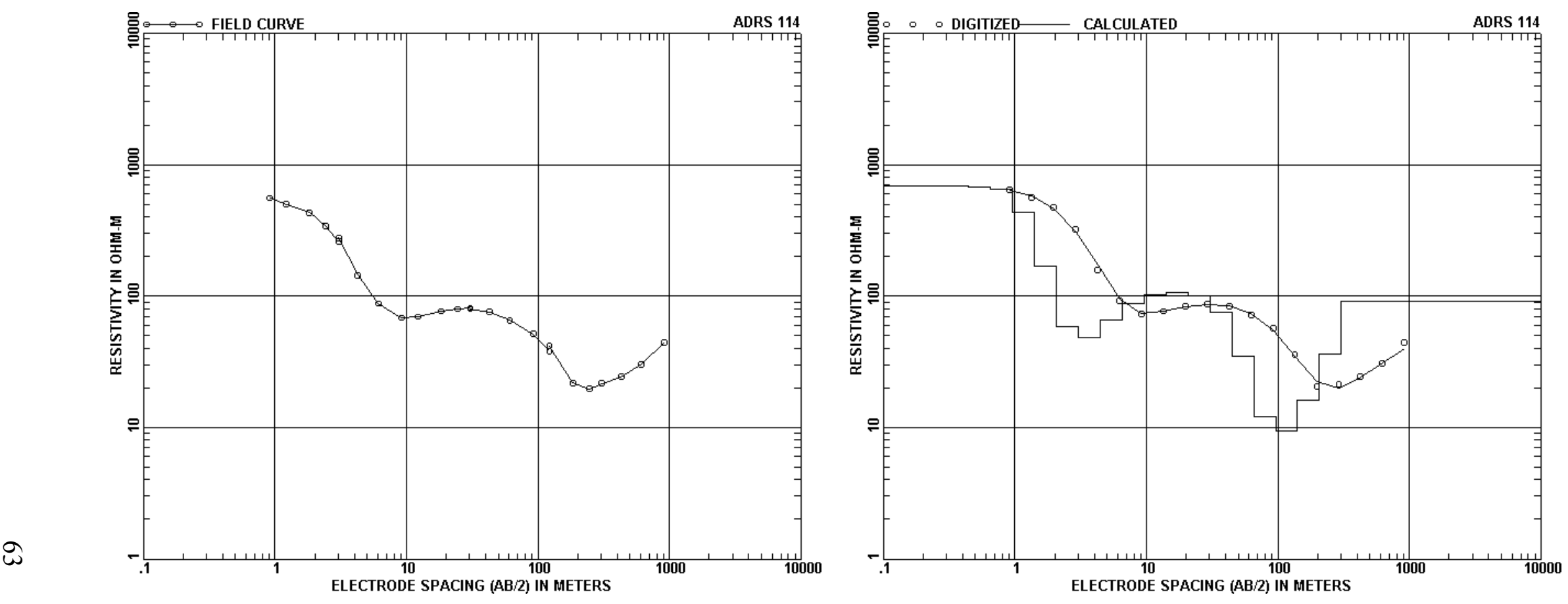

$A B / 2$, $m$ ( ft ) App. Res. $A B / 2$, $m$ ( ft ) App. Res.

DEPTH, $m$ ( ft ) RESIS. DEPTH, m ( ft ) RESIS.

\begin{tabular}{|c|c|c|c|c|c|}
\hline 0.91 & $3.00)$ & 556.00 & 30.48 & $100.00)$ & 81.30 \\
\hline 1.22 & $4.00)$ & 498.00 & 30.48 & $100.00)$ & 79.60 \\
\hline 1.83 & $6.00)$ & 431.00 & 42.67 & $140.00)$ & 75.50 \\
\hline 2.44 & $8.00)$ & 340.00 & 60.96 & $200.00)$ & 65.60 \\
\hline 3.05 & $10.00)$ & 259.00 & 91.44 & $300.00)$ & 51.40 \\
\hline 3.05 & $10.00)$ & 277.00 & 121.92 & $400.00)$ & 38.00 \\
\hline 4.27 & $14.00)$ & 144.00 & 121.92 & $400.00)$ & 41.80 \\
\hline 6.10 & $20.00)$ & 87.90 & 182.88 & $600.00)$ & 21.80 \\
\hline 9.14 & $30.00)$ & 68.30 & 243.84 & $800.00)$ & 19.70 \\
\hline 12.19 & $40.00)$ & 69.60 & 304.80 & $(1000.00)$ & 21.70 \\
\hline 18.29 & $60.00)$ & 76.70 & 426.72 & $(1400.00)$ & 24.30 \\
\hline 24.38 & $80.00)$ & 79.60 & 609.60 & $(2000.00)$ & 30.30 \\
\hline & & & 914.40 & $3000.00)$ & 44.50 \\
\hline
\end{tabular}

$\begin{array}{rlr}0.44 & ( & 1.46) \\ 0.65 & ( & 2.14) \\ 0.96 & ( & 3.14) \\ 1.41 & ( & 4.61) \\ 2.06 & ( & 6.77) \\ 3.03 & ( & 9.93) \\ 4.44 & ( & 14.58) \\ 6.52 & ( & 21.40) \\ 9.57 & ( & 31.41)\end{array}$

685.06

679.46

645.62

437.54

169.78

58.91

47.90

65.44

65.44

87.81
102.76

106.46

100.25

75.85

35.00

12.07

9.46

9.46
16.19

16.19
36.31

36.31 

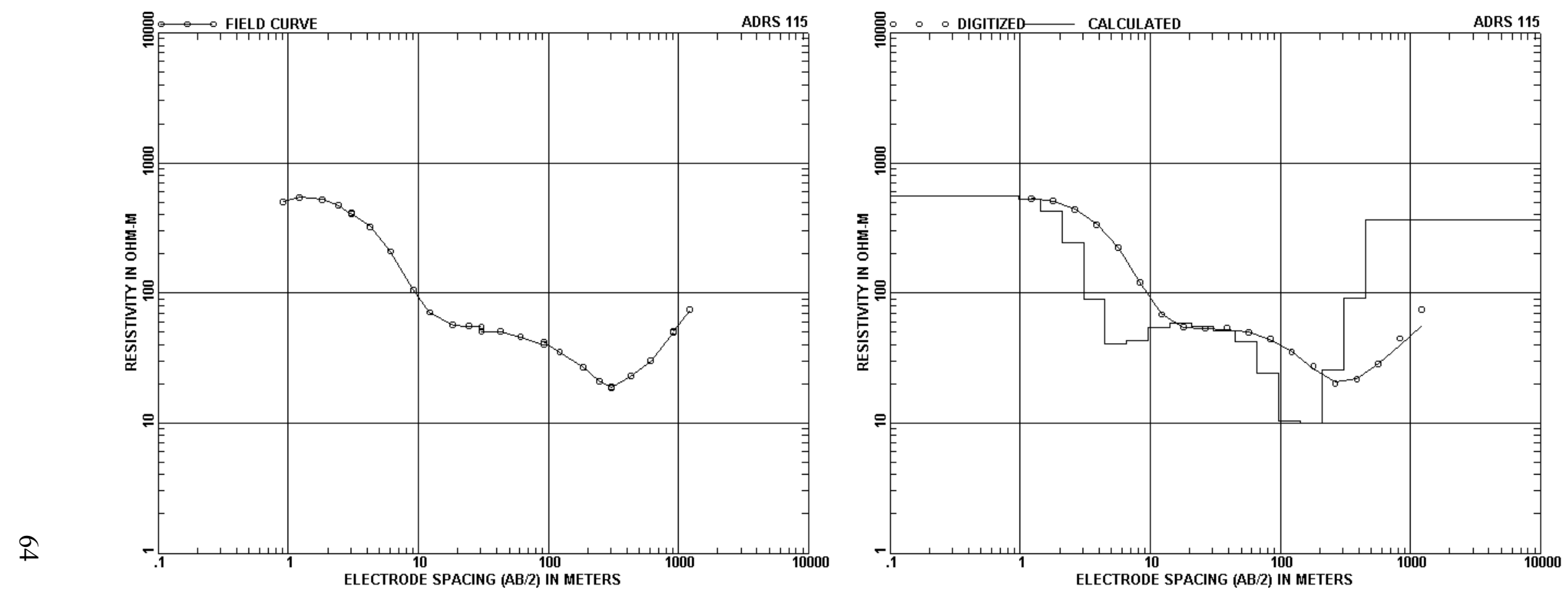

$A B / 2, m$ ( ft ) App. Res. $A B / 2$, m ( ft ) App. Res.

DEPTH, $m$ ( ft ) RESIS. DEPTH, m ( ft ) RESIS.

\begin{tabular}{|c|c|c|c|c|c|}
\hline 0.91 & $3.00)$ & 502.00 & 42.67 & $140.00)$ & 50.60 \\
\hline 1.22 & $4.00)$ & 544.00 & 60.96 & $200.00)$ & 46.00 \\
\hline 1.83 & $6.00)$ & 522.00 & 91.44 & $300.00)$ & 40.20 \\
\hline 2.44 & $8.00)$ & 471.00 & 91.44 & $300.00)$ & 42.00 \\
\hline 3.05 & $10.00)$ & 405.00 & 121.92 & $400.00)$ & 35.10 \\
\hline 3.05 & $10.00)$ & 413.00 & 182.88 & $600.00)$ & 27.00 \\
\hline 4.27 & $14.00)$ & 322.00 & 243.84 & $800.00)$ & 21.00 \\
\hline 6.10 & $20.00)$ & 209.00 & 304.80 & $(1000.00)$ & 19.00 \\
\hline 9.14 & $30.00)$ & 106.00 & 304.80 & $(1000.00)$ & 18.70 \\
\hline 12.19 & $40.00)$ & 71.00 & 426.72 & $(1400.00)$ & 23.00 \\
\hline 18.29 & $60.00)$ & 56.70 & 609.60 & $2000.00)$ & 30.00 \\
\hline 24.38 & $80.00)$ & 55.50 & 914.40 & $3000.00)$ & 50.00 \\
\hline 30.48 & $100.00)$ & 55.00 & 914.40 & $3000.00)$ & 51.00 \\
\hline 30.48 & $100.00)$ & 50.40 & 1219.20 & $4000.00)$ & 74.00 \\
\hline
\end{tabular}

\begin{tabular}{|c|c|}
\hline 0.66 & 2.16 \\
\hline 0.97 & 3.1 \\
\hline 1.42 & 4. \\
\hline 2.08 & 6. \\
\hline 3.06 & 10. \\
\hline 4.49 & 14. \\
\hline 6.58 & 21. \\
\hline 9.66 & 31.7 \\
\hline 4.18 & 46.5 \\
\hline
\end{tabular}

554.22

552.13

523.24

421.63

241.46

89.91

40.64

43.25

54.54
58.38

55.66

51.18

42.23

24.22

10.46

9.98

25.71

25.71

91.02 

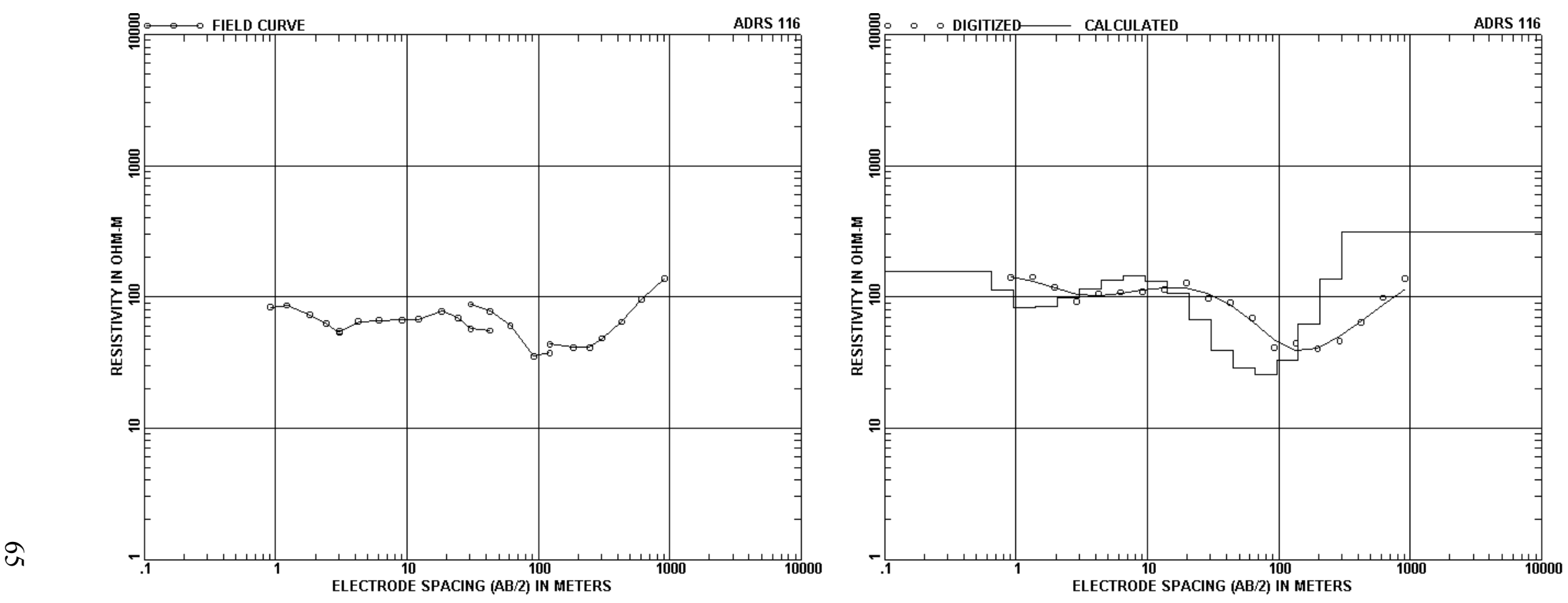

$A B / 2, m$ ( ft ) App. Res. $A B / 2$, m ( ft ) App. Res.

DEPTH, $m$ ( ft ) RESIS. DEPTH, m ( ft ) RESIS.

\begin{tabular}{|c|c|c|c|c|c|}
\hline 0.91 & $3.00)$ & 83.20 & 42.67 & $140.00)$ & 55.20 \\
\hline 1.22 & $4.00)$ & 86.00 & 30.48 & $100.00)$ & 87.90 \\
\hline 1.83 & $6.00)$ & 72.70 & 42.67 & $140.00)$ & 77.70 \\
\hline 2.44 & $8.00)$ & 62.50 & 60.96 & $200.00)$ & 60.60 \\
\hline 3.05 & $10.00)$ & 53.30 & 91.44 & $300.00)$ & 35.20 \\
\hline 3.05 & $10.00)$ & 54.70 & 121.92 & $400.00)$ & 37.50 \\
\hline 4.27 & $14.00)$ & 65.00 & 121.92 & $400.00)$ & 43.80 \\
\hline 6.10 & $20.00)$ & 66.00 & 182.88 & $600.00)$ & 41.20 \\
\hline 9.14 & $30.00)$ & 66.80 & 243.84 & $800.00)$ & 41.30 \\
\hline 12.19 & $40.00)$ & 67.50 & 304.80 & $(1000.00)$ & 48.40 \\
\hline 18.29 & $60.00)$ & 77.50 & 426.72 & $(1400.00)$ & 64.40 \\
\hline 24.38 & $80.00)$ & 69.20 & 609.60 & $(2000.00)$ & 96.10 \\
\hline 30.48 & $100.00)$ & 57.10 & 914.40 & $(3000.00)$ & 138.00 \\
\hline
\end{tabular}

\begin{tabular}{|c|c|c|}
\hline 0.44 & $1.46)$ & 155.37 \\
\hline 0.65 & $2.14)$ & 155.76 \\
\hline 0.96 & $3.14)$ & 112.64 \\
\hline 1.41 & 4.61) & 82.27 \\
\hline 2.06 & $6.77)$ & 84.69 \\
\hline 3.03 & $9.93)$ & 98.96 \\
\hline 4.44 & $14.58)$ & 114.52 \\
\hline 6.52 & $21.40)$ & 134.11 \\
\hline 9.57 & $31.41)$ & 143.48 \\
\hline
\end{tabular}

$14.05(46.11) \quad 132.12$

$20.63(67.67) \quad 105.49$

$30.28(99.33) \quad 67.61$

$44.44(145.80) \quad 39.28$

$65.23(214.01) \quad 28.82$

$95.74(314.12) \quad 25.53$

$140.53(461.06) \quad 32.72$

$206.27(676.74) \quad 62.52$

$302.77(993.32) \quad 137.52$

$99999.00(99999.00) \quad 311.65$

$914.40(3000.00)$

138.00 